\title{
PAST, PRESENT AND FUTURE OF ACTIVE EXPERIMENTS IN SPACE
}

\author{
A. V. Streltsov ${ }^{1,2}$, J.-J. Berthelier ${ }^{3}$, A. A. Chernyshov ${ }^{4}$, V. L. Frolov ${ }^{5,6}$, F. Honary ${ }^{7}$, \\ M. J. Kosch ${ }^{7,8,9}$, R. P. McCoy ${ }^{10}$, E. V. Mishin ${ }^{2}$, M. T. Rietveld ${ }^{11,12}$ \\ ${ }^{1}$ Embry-Riddle Aeronautical University, Daytona Beach, Florida, USA; \\ ${ }^{2}$ Air Force Research Laboratory, Space Vehicles Directorate, Albuquerque, New Mexico, USA; \\ ${ }^{3} L A T M O S / I P S L, C N R S-U P M C-U V S Q, U P M C$, Paris, France; \\ ${ }^{4}$ Space Research Institute, Moscow, Russia; \\ ${ }^{5}$ Nizhny Novgorod State University, Nizhny Novgorod, Russia; \\ ${ }^{6}$ Kazan Federal University, Kazan, Russia; \\ ${ }^{7}$ Lancaster University, Lancaster, United Kingdom; \\ ${ }^{8}$ South African National Space Agency, Hermanus, South Africa; \\ ${ }^{9}$ University of the Western Cape, Bellville, South Africa; \\ ${ }^{10}$ Geophysical Institute, University of Alaska Fairbanks, Fairbanks, Alaska, USA; \\ ${ }^{11}$ EISCAT, Ramfjordbotn, Norway; \\ ${ }^{12}$ UiT The Arctic University of Norway, Troms $\phi$, Norway.
}

\begin{abstract}
Active ionospheric experiments using high-power, high-frequency transmitters, "heaters", to study plasma processes in the ionosphere and magnetosphere continue to provide new insights into understanding plasma and geophysical proceses. This review describes the heating facilities, past and present, and discusses scientific results from these facilities and associated space missions. Phenomena that have been observed with these facilities are reviewed along with theoretical explanations that have been proposed or are commonly accepted. Gaps or uncertainties in understanding of heating initiated phenomena are discussed together with proposed science questions to be addressed in the future. Suggestions for improvements and additions to existing facilities are presented including important satellite missions which are necessary to answer the outstanding questions in this field.
\end{abstract}




\section{Table of Contents}

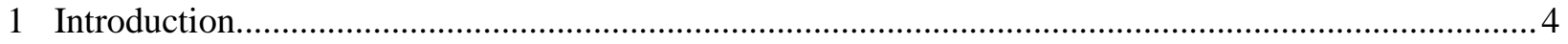

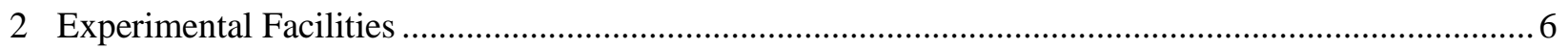

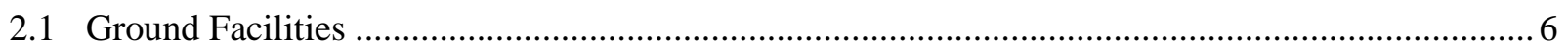

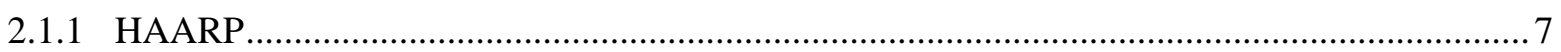

2.1.2 SURA

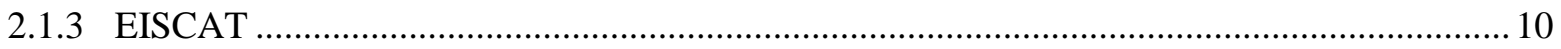

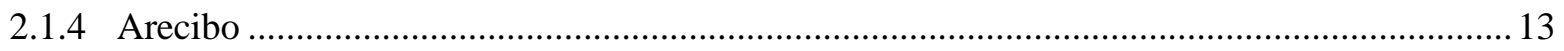

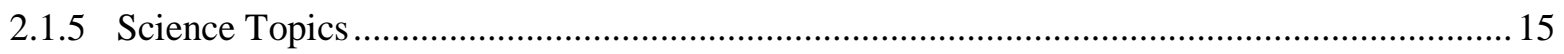

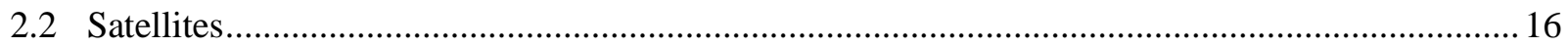

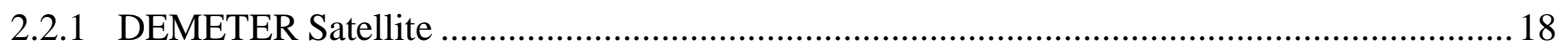

2.2.2 Defense Meteorological Satellite Program (DMSP) .........................................................20

2.2.3 The Demonstration and Science Experiments (DSX) Satellite .............................................22

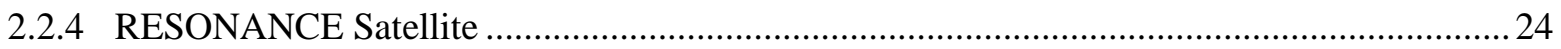

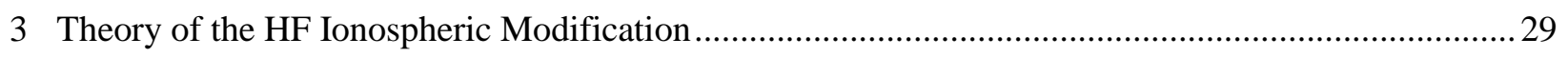

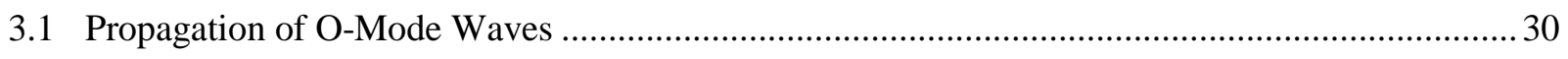

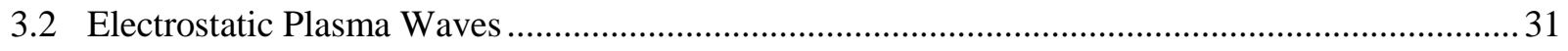

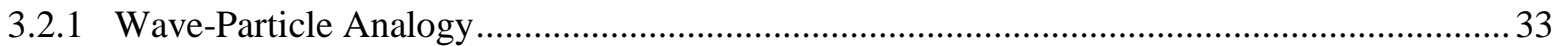

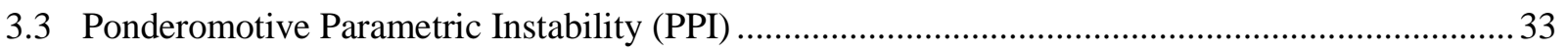

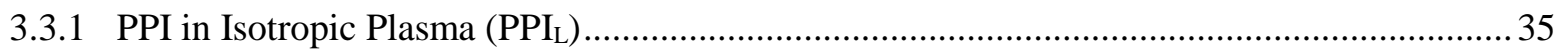

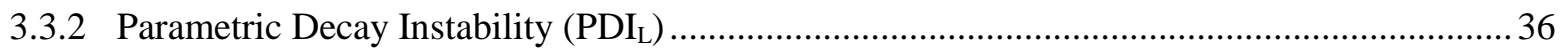

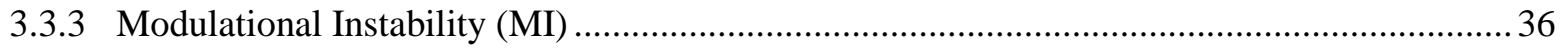

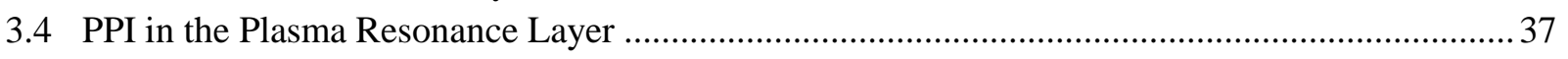

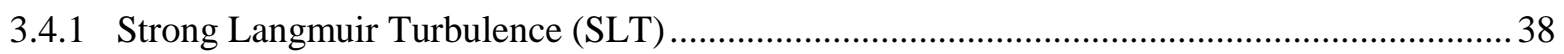

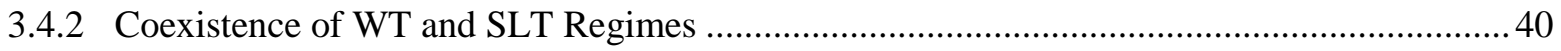

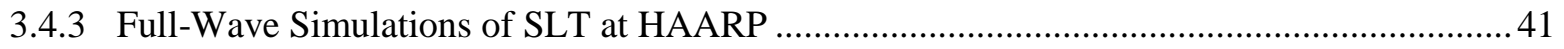

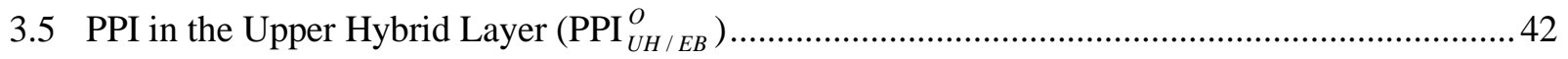

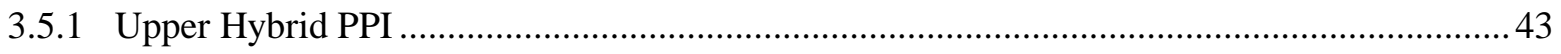

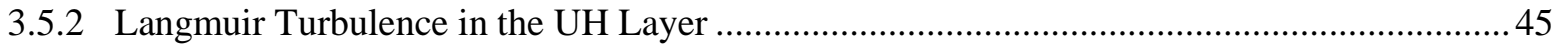

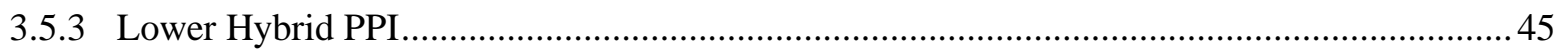

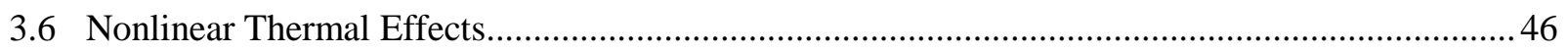

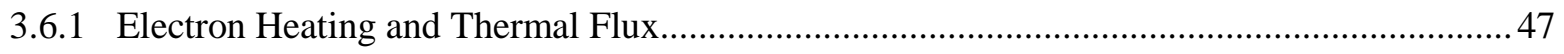

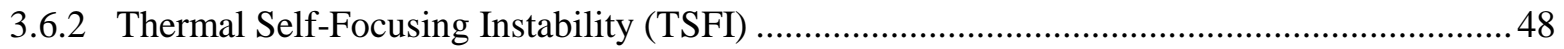

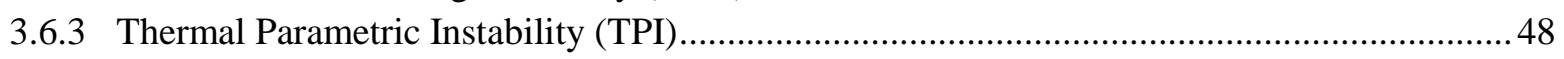

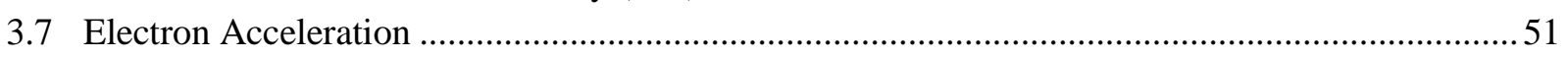

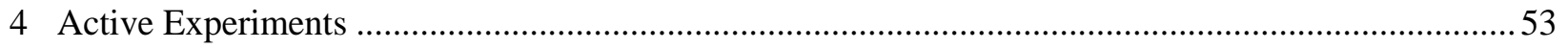

4.1 Stimulated Electromagnetic Emissions (SEEs) …...................................................................53

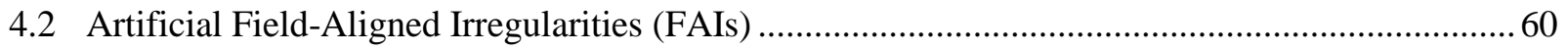

4.2.1 Amplitude-Time History of the Pump Wave Reflected from the Ionosphere ........................64 


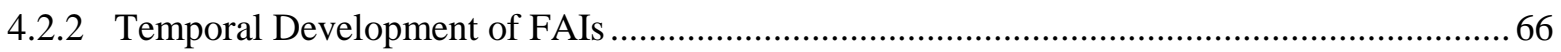

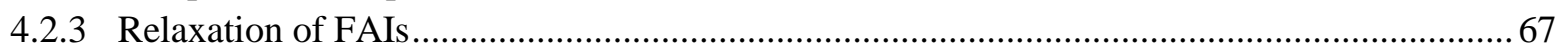

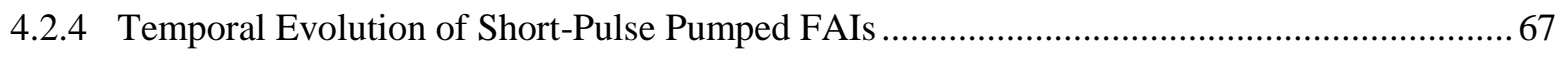

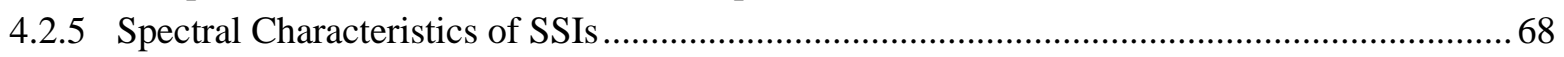

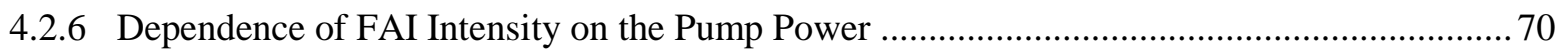

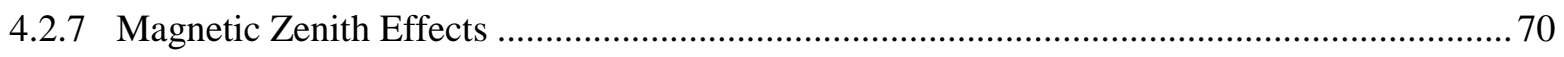

4.2.8 Unexplained UHF Radar Backscatter at the Magnetic Zenith............................................. 72

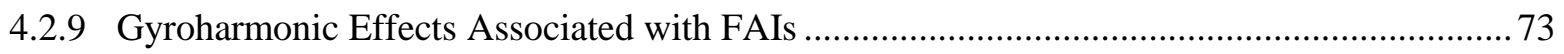

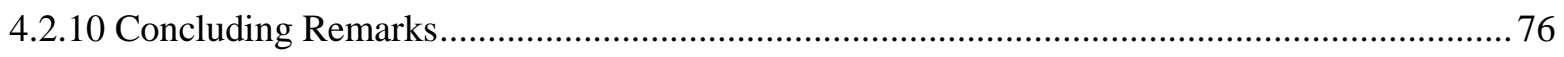

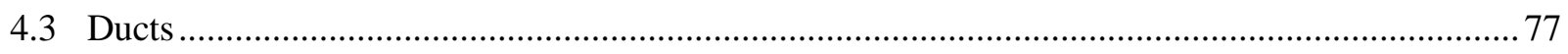

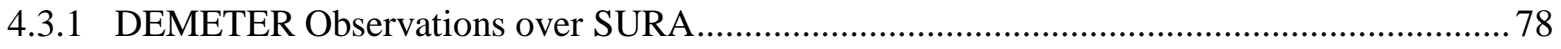

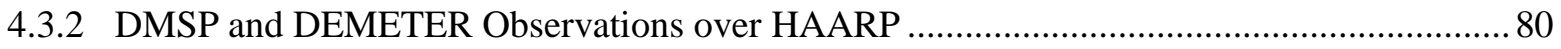

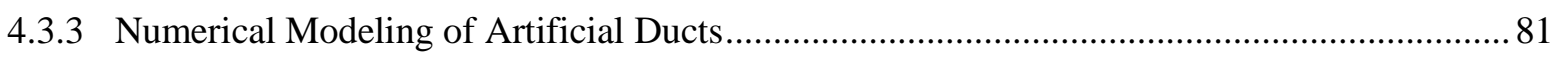

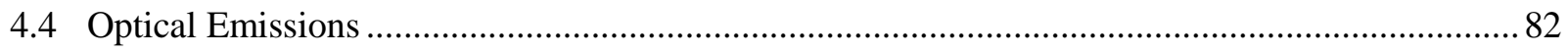

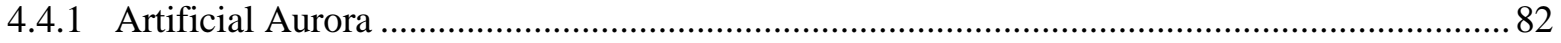

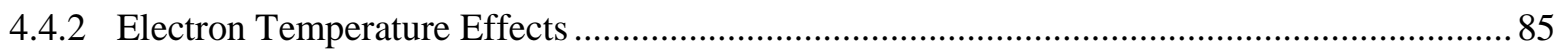

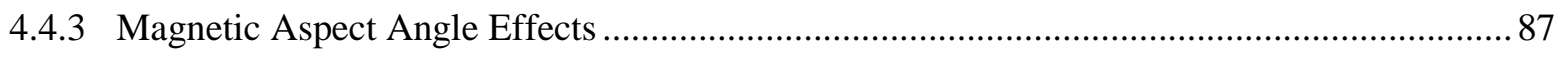

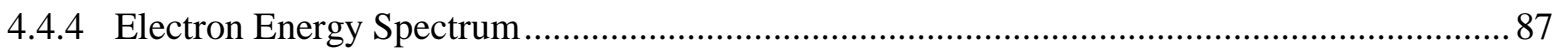

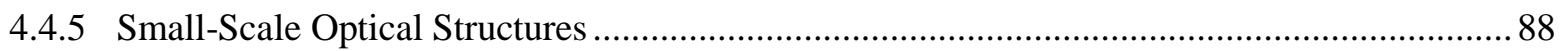

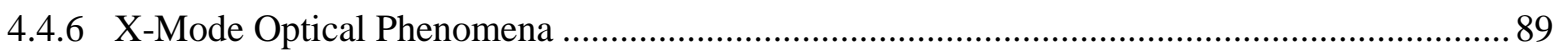

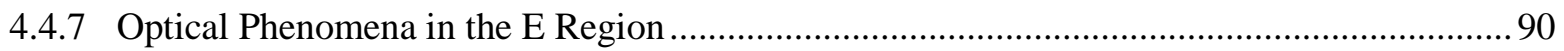

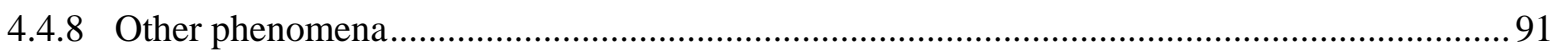

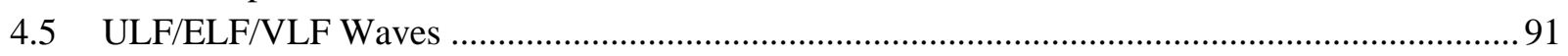

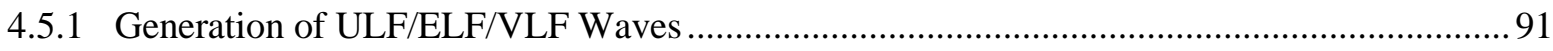

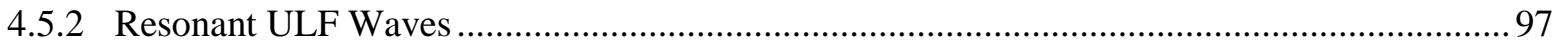

4.5.3 ULF Waves in the Global Magnetospheric Resonator ...................................................... 99

4.5.4 ULF Waves in the Ionospheric Alfvén Resonator ........................................................... 102

4.5.5 ULF Waves in the Earth-Ionosphere Waveguide (Schumann Resonator) ........................... 103

4.5.6 ELF/VLF Waves in the Magnetosphere ....................................................................... 105

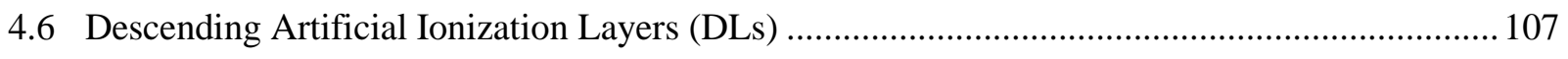

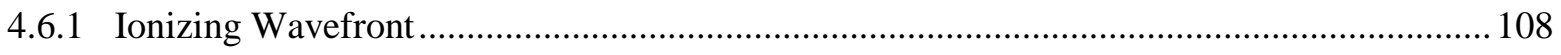

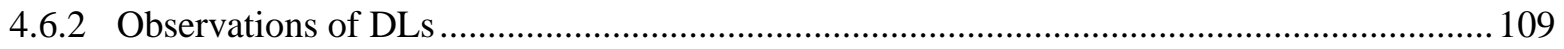

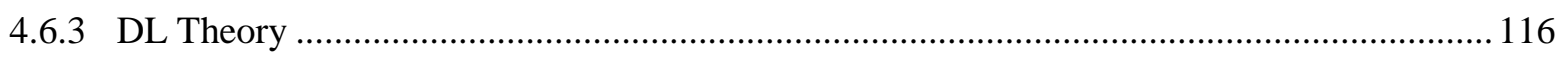

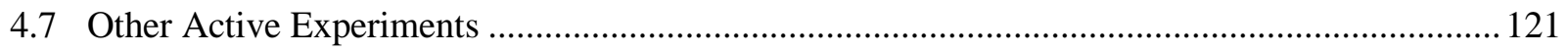

4.7.1 Artificial Ionospheric Horizontal Periodic Irregularities (APIs) ........................................ 121

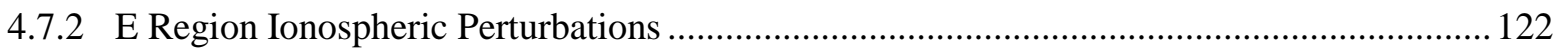

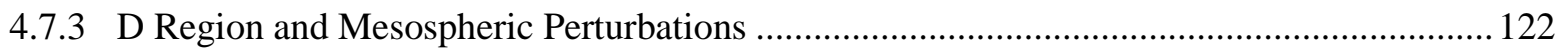

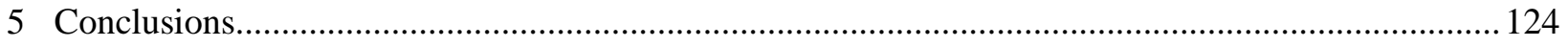

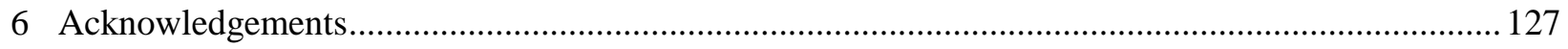

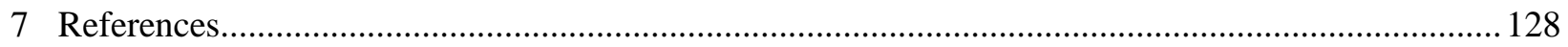




\section{Introduction}

Active ionospheric experiments involving the use of high-power, high-frequency (HF) transmitters to heat small regions of the ionosphere have a tremendous potential to reveal a wealth of information about plasma processes. The Earth's ionosphere is strongly coupled to the sun, less strongly coupled to the earth, is highly variable and exerts a wide array of effects on radio frequency $(\mathrm{RF})$ propagation. Conventional ionospheric investigations involve remote sensing with RF transmitters on the ground or in space, or in-situ measurements with sounding rockets or satellites. To investigate a particular ionospheric phenomena with conventional techniques, the experimenter must wait for that phenomena to appear. Active experiments have the ability to create a desired phenomenon on demand, effectively turning the overhead ionosphere into a plasma laboratory without walls.

This review is concerned with active experiments involving heating of the ionosphere with HF transmitters from the ground and does not address other classes of active experiments involving chemical releases or in-situ plasma discharges or beam injections.High-power, HF radio transmitters can disturb plasma in the Earth's ionosphere and magnetosphere providing a unique opportunity to study interaction between electromagnetic waves and particles without the limited spatial scale-size and chamber edge effects that can be encountered while performing plasma experiments in a laboratory. By modulating the transmitted power in time, space or frequency, the ionosphere can effectively become an antenna for the generation of lower frequency waves. These lower frequency waves (ELF, VLF, ULF) provide opportunities to study a wide array of electromagnetic wave-particle interactions. The resulting interaction between the EM "pump" wave and the ionospheric plasma can then be observed via a number of channels: UHF/VHF incoherent scatter radar measures the plasma density and temperature; optical instruments observe the visible-spectrum of optical emissions produced via suprathermal electron collisions with neutrals; radio receivers and spectrum analysers monitor the stimulated electromagnetic emission (SEE) signal emerging from the heated region.

The topic of active experiments by high power radio waves has generated enormous interest with thousands of publications including review articles by Gurevich [2007], Leyser [2001], and Leyser and Wong [2009]. The focus of this review is to a) report on the theoretical and experimental results from primarily the last decade in the areas of field-aligned irregularities, instabilities, ducts, ionization layers, optical emissions and ULF/ELF wave generation and propagation; b) discuss in detail satellite missions which have directly improved our understanding of new phenomena such as the formation of ducts; and c) to highlight science topics to be explored and propose experiments to address outstanding questions in this field.

Section 2 describes the experimental facilities with section 2.1 giving a brief history and details of the four currently active ground-based heaters together with their main diagnostic instruments. Satellites and rockets have provided unique and important in-situ measurements of the heated volume and disturbances propagating from it to the upper ionosphere and magnetosphere. They are mentioned in some of the sections describing each HF facility. The most recent and planned 
satellites, which are an increasingly important space-based diagnostic of HF ionospheric pumping above all the HF facilities, are described separately in section 2.2.

Ideally heating experiments should be specially designed to test specific hypotheses. This requires a quantitative and comprehensive modelling of linear and nonlinear aspects of wave propagation, wave-wave and wave particle interactions, turbulence and instabilities of many different types in a highly inhomogeneous magnetized plasma. To make this review as selfconsistent as possible, we include in section 3 an overview of the various physical mechanisms that are involved in the most important observed phenomena related to ionospheric heating.

Section 4 reviews recent advances in the ionospheric heating experiments focusing on generation and spatio-temporal properties of 1) stimulated electromagnetic emissions (SEE), 2) artificial ionospheric structures or magnetic field-aligned irregularities (FAI), 3) ducts, 4) optical emissions, also known as artificial aurora, 5) ULF and ELF waves propagating into the magnetosphere and in the earth-ionosphere waveguide, 6) artificial ionization layers.

The conclusions section summarizes the current state of knowledge in the field of ionosphere heating with HF waves of different powers and provides a suggested list of problems to be addressed in the future. 


\section{Experimental Facilities}

\subsection{Ground Facilities}

The first active ionospheric experiment occurred unintentionally when the broadcast from a powerful commercial AM station in Luxembourg could be heard by a receiver tuned to another medium frequency station [Tellegen, 1933]. This "cross modulation", now known as the "Luxembourg Effect", is explained by as little as a 5\% change in electron temperature caused by the powerful modulating station, which modifies the electron collision frequency and hence the absorption of other radio waves traveling through the disturbed region which ultimately superimposes the modulation on them.

The first major HF facility constructed was a $1.45 \mathrm{MHz}$ transmitter near Moscow, Russia which was built to test a hypothesis published by Bailey [1937] that HF radio wave energy at the electron gyro frequency could efficiently accelerate electrons into atomic oxygen atoms to produce visible light. Experiments were performed in 1961 and were not successful, but since the work was highly classified, it was not until it was unclassified in 1973 that researchers were able to explain why the optical emissions were not possible [Gurevich, 2007].

High power ionospheric modification research first appeared in the open literature using experiments in Platteville, USA led by W. Utlaut. Findings were collected in a special issue of Radio Science, vol 9, 1974, based on HF heater induced spread F, field-aligned ionization structure, wide-band absorption, and airglow. It soon became clear that it would be highly desirable to include an incoherent scatter radar in the complement of diagnostic tools. This led to an ionospheric heater being introduced at Arecibo by suspending an HF feed above the primary reflector allowing the $433 \mathrm{MHz}$ incoherent scatter facility to be used to probe the heating effects [Gordon et al., 1971]. Several other relatively low power facilities were subsequently built during the 1970's, such as Monchengorsk, near Murmansk, and Zimenki, near Nizhny Novgorod, Russia.

The present modern class of heater facilities were then built, one at the high latitude location of Troms $\varnothing$, Norway, co-located with the two new EISCAT incoherent scatter radars (ISR) in 1981, and the other at the mid-latitude station, SURA, in Russia. Another high latitude station, HIPAS, was built by the University of California in Alaska [Wong et al., 1990] in the late 1980's and operated from 1986 to 2007. In 1993 the High Frequency Active Auroral Research Program (HAARP) facility was started, reaching completion in 2007 making it the most powerful and advanced facility of all. The highest latitude heater, SPEAR [Robinson, 2006], was built on the island of Spitsbergen and operated from 2004 to 2015, but has since been dismantled.

Today there are four active HF facilities around the world. They are: SURA in Russia [Belikovich et al., 2007], EISCAT heating facility (built and formerly operated by Germany's MaxPlanck Institute) in Norway [Rietveld et al., 2016], HAARP in Alaska, USA [Pedersen and Carlson, 2001], and most recently a new HF facility for the Arecibo $300 \mathrm{~m}$ antenna [Carlson et al., 2017]. Only Arecibo and the EISCAT HF facilities are co-located with incoherent scatter radars (ISRs), although HAARP has a small UHF phased array radar called the Modular UHF Ionospheric Radar (MUIR) which can receive HF-enhanced echoes normally seen with ISRs. In 
section 2.1 these four facilities are described in more detail together with an outline of the major ground-based diagnostics and the research areas these facilities address.

In addition to dedicated HF facilities designed specifically for interactions with the ionosphere, it should not be forgotten that other powerful radio transmitting facilities like ISRs are also capable of heating thermal electrons in spite of their frequencies being much higher than HF. The mechanism is simple Ohmic heating in the collisional lower ionosphere, by the extremely powerful ISR transmissions. This was demonstrated using the Arecibo facility by Sulzer et al. [1982], and will also be possible with the new EISCAT_3D facility under construction in northern Scandinavia [McCrea et al., 2015].

\subsubsection{HAARP}

The High frequency Active Auroral Research Program (HAARP) facility, located in Gakona, Alaska (latitude: $62.39^{\circ} \mathrm{N}, 145.15^{\circ} \mathrm{W}$; magnetic latitude: $63.09^{\circ} \mathrm{N}, 92.44^{\circ} \mathrm{W}$ ) is the world's most powerful and sophisticated facility for active experimentation in the upper atmosphere and ionosphere. HAARP uses powerful HF waves to heat small $(\sim 30-100 \mathrm{~km})$ regions of the upper atmosphere to stimulate particular geophysical processes that can be disentangled by ground-based diagnostic instruments from complex and coupled natural phenomena in the thermosphere and ionosphere. The HAARP facility can indeed create its own natural plasma laboratory without walls in the ionosphere and perform controlled experiments to study a variety of linear and nonlinear plasma physics phenomena that are difficult to capture with satellites or sounding rockets.

HAARP is ideally located to investigate a large variety of geophysical phenomena. The overhead sub-auroral ionosphere can be stable but during even moderately active geomagnetic conditions, the active auroral zone moves above HAARP allowing experiments to be performed within the aurora. Near an L-shell of 5, low frequency waves generated by HAARP can propagate upward along magnetic field lines high into the magnetosphere and even into the conjugate ionosphere.

The primary HAARP transmitter is the Ionospheric Research Instrument (IRI), a phased array of $180 \mathrm{HF}$ crossed dipole antennas covering an area of 33 acres and radiating EM waves in the frequency range 2.8 to $10 \mathrm{MHz}$ with a net power of $3.6 \mathrm{MW}$. The antenna array is fed by transmitters located in 30 electronic shelters (trailers) powered by five $2500 \mathrm{~kW}$ generators, each driven by a $3600 \mathrm{hp}$ diesel engine (4+1 spare). The multiple beams from the phased array can be rapidly reconfigured to achieve

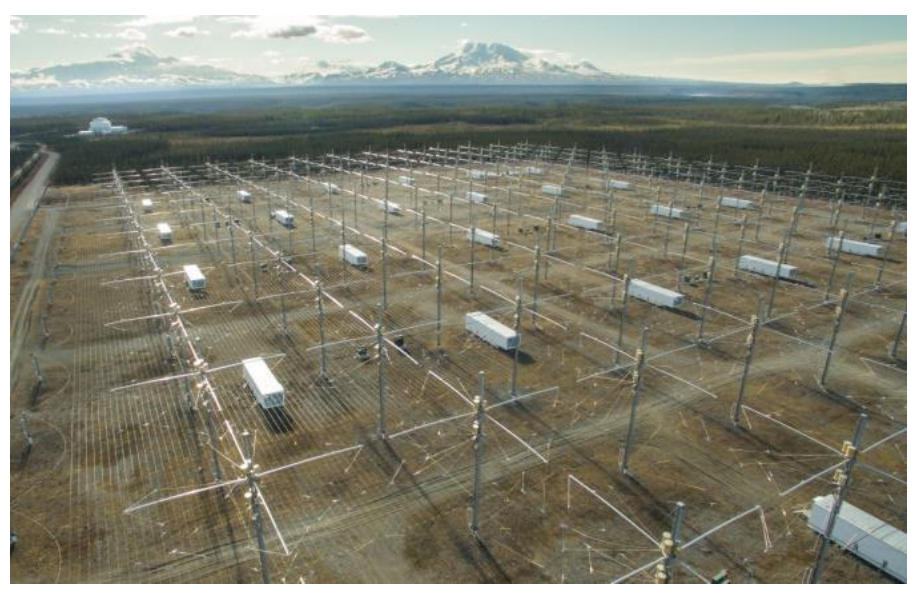

Figure 2.1. HAARP antenna array. Drone photo courtesy of Jessica Matthews. 
complex spatially and temporally variable antenna patterns down to elevations angles of $30^{\circ}$ from the zenith. A photo of the antenna array is shown in Figure 2.1. Because HAARP employs a phased array antenna, energy can be concentrated along variable directions, producing an effective radiated power (ERP) in the few GW range allowing a wide range of unique experiments. Other key instruments at HAARP include an ionosonde, GPS receivers, magnetometers, riometers, optical instruments and the MUIR radar.

The HAARP program was initiated in 1989 and managed by the Air Force Research Laboratory (AFRL) and the Office of Naval Research (ONR). The facility was enhanced with additional funding from the Defense Advanced Research Projects Agency (DARPA), AFRL and ONR. In 2007 HAARP began operating at its current power levels. The ONR interest for HAARP was primarily focused on making the heated ionosphere a many-kilometer long antenna to generate and propagate extremely low frequency (ELF) signals for submarine communications. AFRL interest included studies of over the horizon radar capabilities and using the ionosphere to generate and inject ultra-low-frequency, extremely-low-frequency and very-low-frequency (ULF, ELF, VLF) waves along magnetic field lines into the magnetosphere. The goal was to use these waves to modify the pitch-angle distributions of trapped high energy electrons and increase their precipitation rates in order to reduce their fluxes in the radiation belts.

Additional potential applications of HAARP include the use of the facility for: ionospheric imaging and solar corona/wind sounding; global HF communication and emergency broadcast messages; communication with submarines; detection of the sub-surface cavities; and as a transmitting element of an over horizon radar (OTHR) system.

In 2013 the Space Studies Board of the National Research Council conducted a Workshop to assess the scientific viability of HAARP. The Workshop resulted in a report entitled "The Role of High-Power, High Frequency Transmitters in Advancing Ionospheric/Thermospheric Research." That report described the scientific potential of HAARP to address science topics which are described in Section 2.1.5.

\subsubsection{SURA}

Ionospheric modification experiments in Nizhny Novgorod, Russia have been performed by the Radio Physical Research Institute (NIRFI) since 1973 at the Zimenki heating facility, located $20 \mathrm{~km}$ to the east of Nizhny Novgorod, Russia. This facility was operated at two pump wave

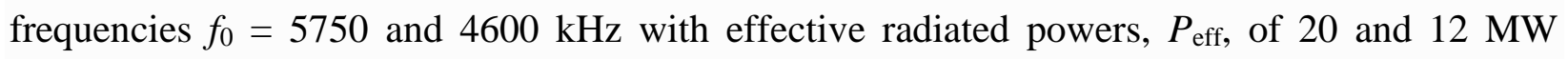
respectively. The experimental results obtained were so impressive that it was decided to build a new more powerful heating facility (SURA facility) near the settlement of Vasil'sursk, $100 \mathrm{~km}$ to the east of Nizhny Novgorod $\left(56.15^{\circ} \mathrm{N}, 46.1^{\circ} \mathrm{E}\right.$; magnetic dip angle $\left.I=71^{\circ}\right)$. The SURA facility was put into operation in November 1980. Since then it has been used for ionosphere modification by $\mathrm{HF}$ radio-waves to investigate a range of science topics listed in section 2.1.5. 
A comprehensive description of the SURA facility can be found in Belikovich et al. [2007]. The facility comprises three HF broadcast transmitters. Each of them has a maximum output power of $250 \mathrm{~kW}$, within a frequency range from 4 to $25 \mathrm{MHz}$. Tuned to the pump frequency, the transmitter bandwidth is about $50 \mathrm{kHz}$. Each transmitter is connected to a subantenna array containing 4 rows of 12 wideband crossed dipoles, which have a bi-conical form. A section of the SURA antenna array is shown in Figure 2.2. It allows radiating either left or right circular polarized waves (O- or X-mode waves) from 4.3 to $9.5 \mathrm{MHz}$, covering a frequency range from slightly above the third to above the seventh electron cyclotron harmonic. The size of such a subantenna array is $100 \mathrm{~m}$ in the North-South direction and $300 \mathrm{~m}$ in the East-West direction.

One transmitter together with its subantenna array forms one module of the facility. The three modules of the SURA facility can operate either independently (each with independent frequency, power, polarization, and

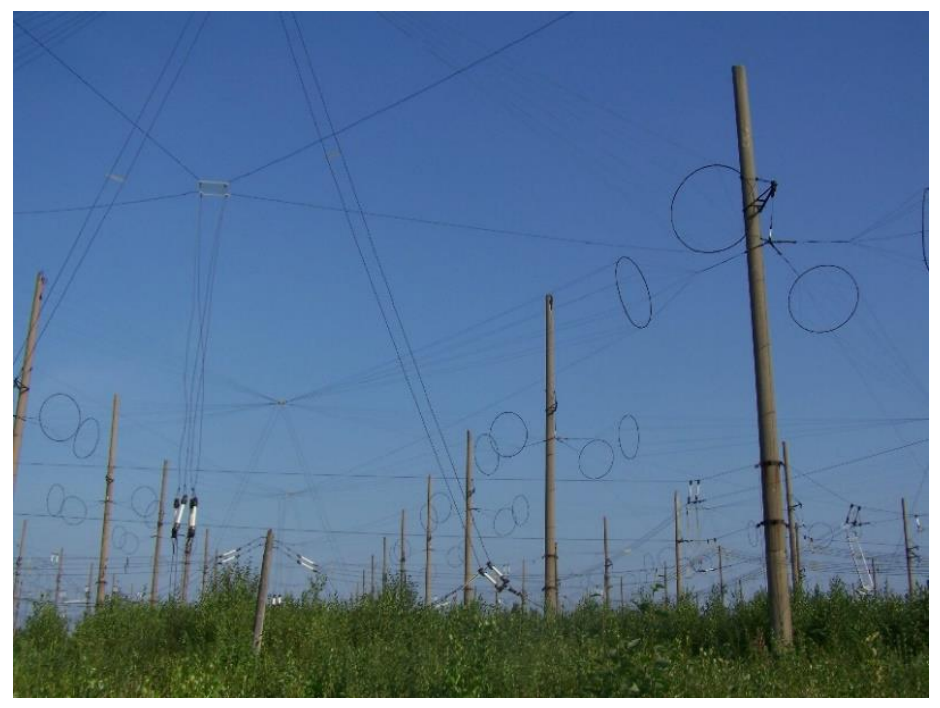
timing), or coherently combining $12 \times 12$ crossed dipoles into one array. In the

Figure 2.2. A view of the SURA antenna array. latter case, the antenna gain, G, ranges from 140 at $4.3 \mathrm{MHz}$ to 330 at $9.5 \mathrm{MHz}$, corresponding to an effective radiated power of 100 to $240 \mathrm{MW}$. In a pulse mode transmission, the lower limit for the length of a pump wave pulse is about $50 \mu$ s. There is no duty cycle limit, so "on"-times can be hours. The beam width for the full antenna array is about $12^{\circ}$ at a frequency of $4.3 \mathrm{MHz}$ decreasing to $6^{\circ}$ at a frequency of $9.5 \mathrm{MHz}$. It is also possible to combine any two facility modules (that gives an antenna array of $8 \times 12$ crossed dipoles), which can independently operate together with the third facility module. In experiments at the SURA facility, such a scheme is often used in so-called additional pumping measurements when two modules are used for ionosphere pumping and the third module is used to induce stimulated electromagnetic emission (SEE) for diagnostics of plasma processes. The antenna array system was constructed to operate in the following modes: (1) transmitting, (2) receiving, and (3) as a mono-static/bi-static HF radar. The HF beam can be scanned in a geomagnetic meridian plane over the range of $\pm 40^{\circ}$ from the vertical. The main pump wave parameters such as frequency, polarization, beam direction, radiated power, and the configuration of the facility modules are chosen and set up at the time of tuning up. Changing the beam direction or polarization requires about $20 \mathrm{~min}$.

The diagnostic equipment at the SURA facility includes: 
a. HF receiving station comprising a wideband antenna array (16 crossed dipoles with a frequency band from 3 to $6 \mathrm{MHz}, \mathrm{G} \approx 30$ ), HF receivers with digital data registration, a HP-3585A spectrum analyzer, and a wideband digital receiver.

b. Station for sounding the ionospheric D, E, and F regions by means of artificial periodical irregularities (API).

c. Three-channel receiving system to measure amplitude variations of low orbital satellite beacon signals at two frequencies of 150 and $400 \mathrm{MHz}$.

d. GPS/GLONASS receiver to measure HF-induced TEC variations.

e. Station for $\mathrm{HF}$ chirp sounding operating in the 2.7 to $30 \mathrm{MHz}$ frequency range at rates of 0.1 to $1.0 \mathrm{MHz} / \mathrm{s}$.

f. Station for receiving ELF-VLF-ULF signals of natural and artificial origin.

g. Optical instruments for measuring HF-induced airglow.

h. Ionosonde of CADI type.

This equipment allows investigating pump wave self-action effects, measuring SEE features, investigating temporal evolution and spectral characteristics of artificial irregularities with $l_{\perp} \geq 30$ $\mathrm{m}$, to studying long-distance HF propagation effects in the ionosphere, characterizing the $\mathrm{D}, \mathrm{E}$, and $\mathrm{F}$ regions and their dynamics, and studying features of ELF/VLF/ULF emissions. During heating campaigns field-aligned scattering measurements are conducted at receiving stations located near Kazan, Moscow, St.-Petersburg, and Rostov-on-Don, as well as radio tomography measurements at 3 receiving points located near the SURA facility. To study features of plasma perturbations in the outer ionosphere using such satellites as DEMETER, DMSP, CASSIOPE/ePOP, and SWARMs, ionosphere heating sessions were carried out when the satellites crossed a HF-disturbed magnetic flux tube connected to the ionospheric disturbed volume over the SURA facility and its magnetically conjugate location.

The SURA facility can operate in both mono-static and bi-static radar mode. In the latter case it conjugates with either UTR-2 (Kharkov, Ukraine), which is the largest HF radio telescope in the word, or with a receiver placed on a satellite. The SURA facility has been used as a HF radar devoted to sounding the Earth's atmosphere, the near Earth's space, the Sun, and the Moon, as well as for calibration of different HF-systems on satellites.

The SURA antenna array also can be used as a radio astronomical receiving antenna to measure radio emissions from space and discrete radio sources in the frequency range of 5 to $9.5 \mathrm{MHz}$.

\subsubsection{EISCAT}

The Troms $\varnothing$ heating facility was built by the Max-Planck-Institut für Aeronomie at the end of the 1970's, about the same time as the major Russian (SURA) and US HF heating facilities (Arecibo) were being built. Officially opened in 1980, the facility delivered many new results in this early phase of experiments which were summarised in Stubbe et al., [1982, 1985] and Stubbe [1996]. In 1992 the facility was transferred to the EISCAT Scientific Association. The EISCAT $\mathrm{HF}$ facility is co-located with two ISRs at $224 \mathrm{MHz}$ and $930 \mathrm{MHz}$ as well as a $56 \mathrm{MHz}$ 
mesosphere-stratosphere-troposphere (MST) radar. These ISR's will be replaced by a new generation phased array radar at $233 \mathrm{MHz}$, called EISCAT-3D [McCrea et al., 2015] in 2021. The Troms $\varnothing$ HF facility has been described in various publications [Stubbe et al., 1982; Rietveld et al., 1993] but most recently in Rietveld et al. [2016].

The transmitters have not changed at all since they were completed in 1980 . There are 12 vacuum tube transmitters of $100 \mathrm{~kW}$ continuous power operating in class $\mathrm{AB}$ mode covering the range 2.7 to $8.0 \mathrm{MHz}$ but the present antennas only allow use of frequencies between 3.85 and $8 \mathrm{MHz}$. Ageing of the transmitter tubes means that $80 \mathrm{~kW}$ is the normally used maximum power per transmitter in recent years. A photo of the main amplifier of one transmitter is shown in Figure 2.3.

Each transmitter can be connected to one of three antenna arrays. These three antenna arrays and transmission line system are exactly the same as described in [Rietveld et al., 1993] which show the electrical details. The phases at the antennas in each east-west row are fixed by the coaxial cable lengths between the antennas and are set for a vertical radiation pattern. By varying the transmitter phases, one can change the phase between adjacent rows in Array-2 (3.85-5.6 MHz, 22-25 dBi gain) and Array-3 (5.4-8.0 MHz, 22-25 dBi gain) to allow steering of the beam in the north-south (geographic) plane out to about $\pm 30^{\circ}$ from vertical. The original Array-1 which covered the frequency range 2.7-4.1 MHz was destroyed in a storm in October 1985. It was rebuilt in 1990 such that that it also covers 5.4-8.0 MHz but with four times the number of antennas and area of Array-3, resulting in 28-31 dBi gain. Adjacent pairs of antenna rows are connected to one transmitter which limits the beam steering to about $\pm 20^{\circ}$ from vertical, the exact angle depending on frequency. Near these limits grating sidelobes become very strong.

Frequency stepping can be performed rapidly by incrementing through a list of frequencies loaded into the exciter memory. Here it is desirable to keep the frequency steps small enough (usually a few $\mathrm{kHz}$ to about $20 \mathrm{kHz}$ ) such that the automatic tuning and antenna matching circuits in the transmitter can adjust to the new frequency. Phase changes such as for coding a radar transmission pulse and amplitude modulation for pulse shaping or low

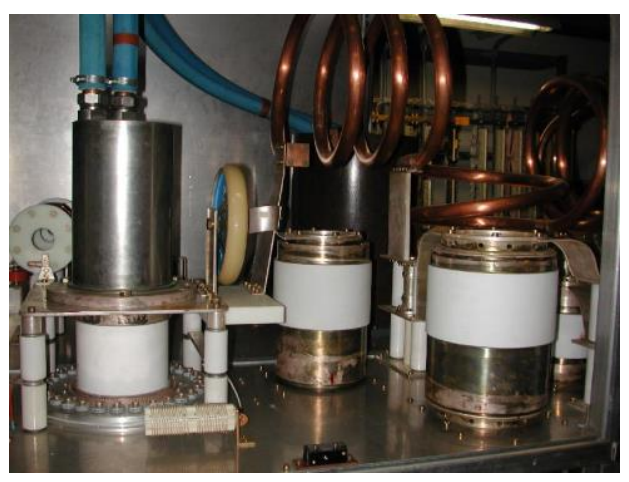

Figure 2.3. The main amplifier of one transmitter at EISCAT frequency modulation can also be made by putting them into similar lists. The shortest dwell time is $1.1 \mu$ s if only amplitude or phase is being changed and $3.7 \mu$ s if amplitude, frequency and phase are all being updated.

The diagnostic equipment at the EISCAT HF facility includes:
a. $933 \mathrm{MHz}$ and $224 \mathrm{MHz}$ incoherent scatter radars.
b. $56 \mathrm{MHz}$ MST radar (MORRO) from the University of Troms $\varnothing$.
c. HF sounders (Dynasonde and Digisonde). 
d. Optical equipment for passive observations: All sky cameras; The Auroral Large Imaging System (ALIS).

e. Magnetometers.

f. SuperDarn HF coherent radars (CUTLASS) in Finland and Iceland.

g. HF receivers for SEE measurements.

One potentially interesting area of research at EISCAT is transmitting at the second electron gyrofrequency. Originally, the heater was built to transmit from $2.75 \mathrm{MHz}$ to $4.04 \mathrm{MHz}$ on Array1 , which allowed operation at the second gyroharmonic $(2.75 \mathrm{MHz}$ at $200 \mathrm{~km})$. This capability was lost when that antenna array was rebuilt to cover a higher frequency range after a catastrophic storm that destroyed most of the feed towers on 25 October 1985. Since then, experiments at HAARP have shown that the second gyroharmonic is indeed of special interest (see sections 4.2.9, 4.4 and 4.6) in that it produces electron acceleration leading to stronger than normal RF-induced optical emissions [Mishin et al., 2016 and references therein]. It would be of great scientific interest to perform such experiments at EISCAT again because of the unique incoherent scatter radar diagnostics available. The transmitters are capable of transmitting this frequency but none of the antenna arrays are. In Array-1 the $22 \mathrm{~m}$ wooden masts that supported the outer ends of the original $6 \times 6$ crossed full-wave low frequency dipoles at a quarter wavelength above the ground still exist. It might be possible to design a simpler array with a limited number of narrow-band antennas (say 3 or 6 per transmitter) above the existing high frequency antennas which are at $12 \mathrm{~m}$ height.

\section{High-Power HF Radar at EISCAT}

The HF facility was not originally intended to operate as a radar. There are at least two areas of research that, however, would benefit from a high-power HF radar co-located with the EISCAT incoherent scatter radars. The first is the application as a mesosphere and possibly stratospheretroposphere radar. Another, more uncertain but potentially more interesting area, is to search for magnetospheric echoes, i.e. echoes coming from above the $\mathrm{F}$ region peak out to perhaps thousands of kilometres, associated with auroral ion-acoustic waves which have been observed at 224, 500 and 933 MHz [Rietveld et al., 1991; Sedgemore-Schulthess and St. Maurice, 2001; Schlatter et al., 2015]. These have been called NEIALS (Naturally Enhanced Ion Acoustic Lines). If similar echoes were obtained at $8 \mathrm{MHz}$ corresponding to $38 \mathrm{~m}$ wave structures, from along the magnetic field line at high altitude, it would provide a new wavelength to study these still-poorly understood echoes which are connected with the aurora and in particular the auroral acceleration region. First attempts were made by Senior et al. [2008] using the HF facility as a transmitter and a simple dipole as receiving antenna. A more sensitive system with direction finding in the north-south plane is now available. Since there are two arrays with different gains/beamwidths capable of operating between 5.4 and $8 \mathrm{MHz}$ by using the high gain $(\sim 30 \mathrm{dBi})$ Array- 1 for transmission, the lower gain Array-3 (24 dBi) can be used as a receiving antenna without the need for the complication of high power transmit/receive switches. Experiments using this new capability have only just started. 


\section{The New EISCAT_3D Radar}

With the planned EISCAT_3D radar [McCrea et al., 2015] both UHF and VHF incoherent scatter radars at Ramfjordmoen will be replaced by a new phased array tristatic radar at $233 \mathrm{MHz}$ with the transmitter located near Skibotn, about $52 \mathrm{~km}$ east southeast of Ramfjordmoen. The construction of the first phase of this project started on 1 September 2017. It is envisioned that heating operation will continue for some time when EISCAT_3D comes on line, around 2021. The geometry is not optimal for some heating experiments, especially since the HF beam cannot be tilted in the east-west plane. For mesospheric heating experiments the EISCAT_3D radar will need to observe at $33^{\circ}$ from the zenith, which should be possible, but at reduced power. It will not be possible to observe with EISCAT_3D along the magnetic field in the heated region so that the wide altitude extent enhanced ion lines (section 4.2.8) cannot be studied in detail. It is not practical to move the present, 36-year old facility nearer Skibotn. So we recommend that a new HF facility will be built nearer Skibotn to exploit the three-dimensional capabilities of the new incoherent scatter radar. This could be done in a staged process, building for example a large number of solidstate HF transmitters which could be connected more or less directly to each antenna in a $12 \times 12$ array.

\subsubsection{Arecibo}

The Arecibo heater has undergone several major changes since the first experiments were performed there in 1970. Mathews [2013] gives a historical description of the heating facility and the radar for the fiftieth anniversary of the observatory. Although the heater has always had relatively modest power compared to some of the other facilities, Arecibo with its more than 100 times more sensitive radar compared to most other incoherent scatter radars [Isham et al., 2000], together with the fact that many HF-induced plasma wave interactions require only modest field strengths to be excited, has made results from this famous facility extremely important. Isham et al. [2000] give a summary of important results as well as the new capabilities after major upgrades from both the ISR and the previous heating facility at the time.

The review and tutorial paper by Djuth and DuBois [2015] gives an excellent summary of the various stages of the Arecibo heater and the state of knowledge about Langmuir wave turbulence results and theory. Similarly, the paper by Carlson et al. [2017] provides a good background to some of the aeronomical issues associated with electron acceleration and compares the results between high and mid-latitudes.

\section{The Arecibo HF Facility}

The new HF facility at Arecibo started tests in 2015 and scientific campaigns in November of 2016. It transmits a maximum of $600 \mathrm{~kW}$ at $5.1 \mathrm{MHz}$, with $22 \mathrm{~dB}$ of gain (95 MW ERP) and $13^{\circ}$ of half power beam width, or $8.175 \mathrm{MHz}$ with $25.5 \mathrm{~dB}$ and $8.5^{\circ}$. The HF transmission has a Cassegrain design where the primary is the $300 \mathrm{~m}$ Arecibo dish, the secondary is a sub-reflector 
mesh that reflects frequencies lower than $20 \mathrm{MHz}$, and the feed system is composed of an array of three concentric cross dipole antennas at each frequency. The transmitters are connected to the antenna arrays by heliax lines. Control of the power gain allows ramping up and down the transmitted power in $\mathrm{dB}$ steps. The system points vertically and supports linear, $\mathrm{O}$ and $\mathrm{X}$ modes, transmitting CW, pulses, AM and FM modes. Figure 2.4 shows the HF antennas in the center of the reflector.

One of the advantages of performing experiments at the HF facility at Arecibo is the extensive diagnostic capabilities, which include:

- $430 \mathrm{MHz}$ Incoherent Scatter Radar

The $430 \mathrm{MHz}$ Incoherent Scatter Radar (ISR) is capable of extremely sensitive diagnostics for HF experiments. It can run in parallel with the HF system, being one of the essential tools for diagnostics of the ionosphere modification over Arecibo. The minimum HF power needed to generate enhanced ion lines detectable by the Arecibo ISR is $125 \mathrm{~kW}(21 \%$ of maximum $\mathrm{HF}$ power) and maintained with $55.8 \mathrm{~kW}$ (10\% of maximum HF power). ISR raw data can be collected with a $25 \mathrm{MHz}$ wide data taking system for later analysis while a narrower bandwidth system is used to provide online monitoring. The current ISR coding technique allows $300 \mathrm{~m}$ range resolution for the enhanced plasma line, ion line and natural plasma line data. Ion and plasma line profiles are normally provided from altitudes as low as $90 \mathrm{~km}$ up to $1000 \mathrm{~km}$. Ion and electron temperatures, ion drifts, ion composition, electric fields and other variables are estimated under user demand.

\section{- Optical Capabilities. The Arecibo} Observatory has active and passive optical
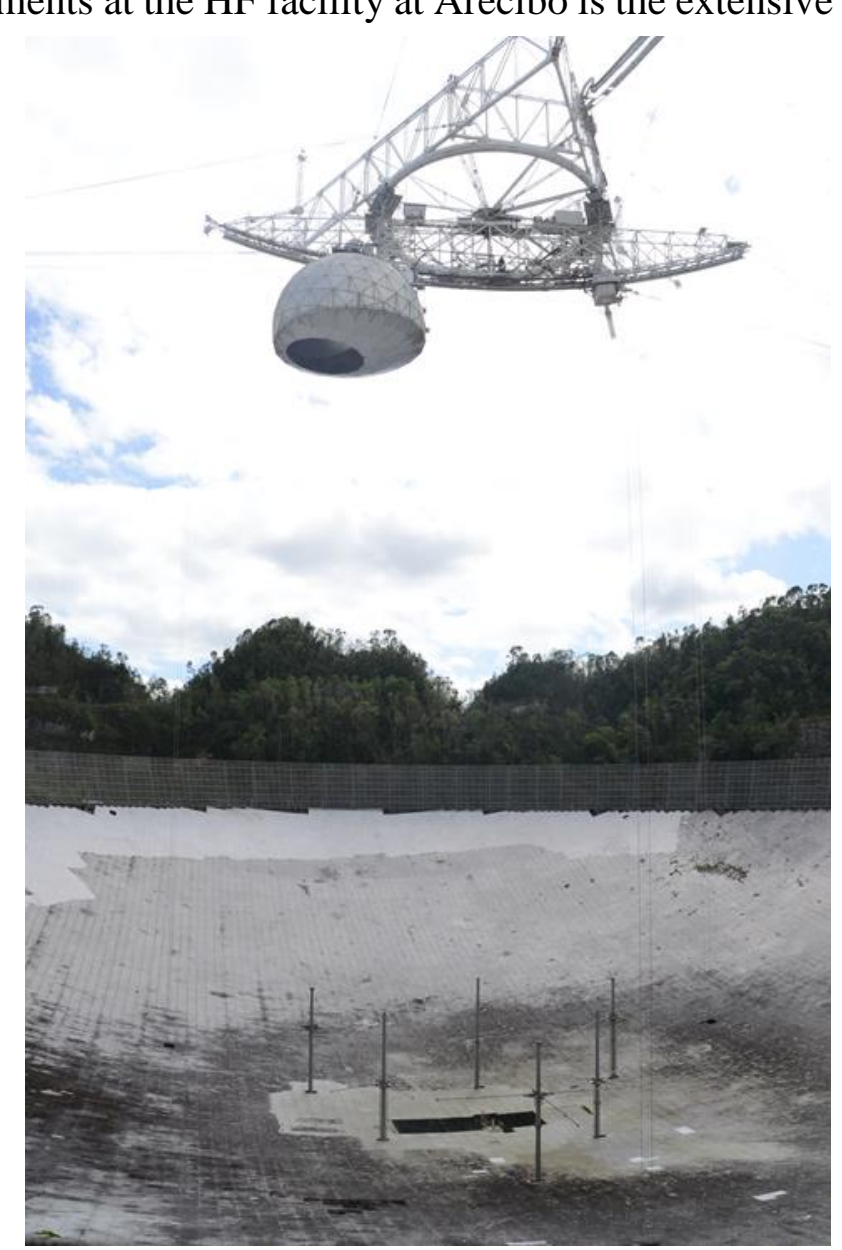

Figure 2.4. The HF antennas in the center of the Arecibo reflector. A wire mesh $\mathrm{HF}$ subreflector, not easily resolvable here, hangs under the platform on top of the HF antenna. The sub-reflector altitude is adjusted according to the selected HF frequency.

instrumentation. The optical instrumentation on-site observes the same volume as the HF system. "Active" optical instruments (lidars) monitor the upper stratosphere to lower thermosphere. There are three systems, two of which are configurable to observe one each of the meteoric metals: $\mathrm{Na}$, $\mathrm{Fe}, \mathrm{Ca}$, or $\mathrm{Ca}^{+}$. Alternatively, one of the two metal lidars can be configured as a Rayleigh lidar to measure temperature from the upper stratosphere to the mesosphere, from about 35 to $70 \mathrm{~km}$. The third lidar is a Doppler-resonance lidar that measures temperatures within the metal layer by 
sensing the Doppler broadening in the D1 resonance line of potassium. The "passive" optical instrumentation located on-site includes monitors the ionosphere emissions using tilting-filter photometers $(630.0 \mathrm{~nm}$ and $555.7 \mathrm{~nm})$, Fabry-Perot interferometers $(630.00 \mathrm{~nm}, 557.7 \mathrm{~nm}$ and $844.6 \mathrm{~nm})$, and an all-sky imager system (630.0 $\mathrm{nm}$ and $643.4 \mathrm{~nm}$ filters).

- Other Radio Instrumentation. The Arecibo Observatory also has a cadi ionosonde, riometers, GPS systems and a software-defined radio system, which are available on demand for the time of the experiments.

- User Instrumentation. The Arecibo Observatory hosts a variety of instrumentation on-site, on the Culebra Island facility, and around the Puerto Rico Island. Among others, the user-based instruments include all-sky airglow imagers, GPS, SEE, high-frequency receivers. Some of these instruments share the data on public databases while others on demand.

The science covered using the HF heaters at Arecibo includes many of the topics in Section 2.1.5. The first satellite studies of HF-induced irregularities and HF self-focusing were made by Farley et al. [1983] using the AE-C satellite. In more recent times rockets flown through the heated region provided detailed measurements of small and medium scale irregularities [Kelley et al., 1995]. There is a rich history and extensive literature concerning observations of Langmuir wave excitation mostly performed with the $430 \mathrm{MHz}$ incoherent scatter radar but also at $46.8 \mathrm{MHz}$ [Fejer et al., 1983]. Important aeronomical studies of artificial ionization are now being made again at Arecibo [Carlson et al., 2017].

\subsubsection{Science Topics}

The science areas that can be explored using heating facilities can be categorized as follows:

\section{Radio Science}

a. Creation of artificial plasma layers \& effects on propagation of HF, UHF waves.

b. Generation of ULF, ELF and VLF \& propagation studies.

c. Creation of artificial irregularities and effects on UHF ground to satellite propagation.

d. Stimulated electromagnetic emission (SEE) effects.

e. Luxembourg effect.

f. Ionospheric radio propagation

\section{Mesosphere and Thermosphere Science}

a. Generation of artificial periodic irregularities and studies of neutral density and temperature effects in the D, E and F regions.

b. Generation of artificial airglow.

c. Electron acceleration by HF-induced Langmuir turbulence.

d. Thermospheric heating to create density plumes and neutral waves: Travelling Ionospheric Disturbances (TIDs), Acoustic Gravity Waves (AGWs) and infrasound waves.

e. Diffusion and cooling rates and $\mathrm{E} \times \mathrm{B}$ drifts.

f. Triggered Emissions. 
g. Studies of polar mesospheric clouds.

h. Sporadic E ionization layers.

i. Mesosphere/themosphere coupling.

Space Weather Studies and Comparisons Inside and Outside the Auroral Zone

a. Studies of subauroral polarization stream (SAPS)/subauroral ion drift (SAID)-related outflows.

b. Studies of auroral substorms, and their possible triggering.

c. Chemistry triggered by high electron temperature and density troughs.

d. Atmospheric gravity waves induced by high-temperature ion-outflow.

\section{Magnetosphere and Radiation Belt Science}

a. Using "virtual antennas," to inject whistler, shear Alfvén, and magnetosonic waves in the magnetosphere and the radiation belts and ULF/ELF/VLF waves in the Earth-ionosphere waveguide.

b. Science of triggered emissions, propagation characteristics, attenuation rates, mode conversion effects of whistler and Alfvén waves.

c. Formation od artificial ducts.

d. Pitch angle scattering of trapped particles on whistler, Alfvén and EMIC waves.

e. Excitation of field line resonances and studies of ionospheric and magnetospheric wave guides and resonators.

f. Possible influence on generation of auroral kilometric radiation (AKR)

\section{Laser Fusion}

a. Nonlinear plasma experiments in unbounded plasma.

b. Investigation of parametric instabilities and nonlinear plasma physics relevant to fusion environments.

Most of the science topics listed above can be investigated by any of the active facilities, but clearly there are differences in the science that can be addressed at the high latitude facilities (HAARP and EISCAT) and the mid- (SURA) and low latitude (Arecibo) facilities. For example, PMSE and PMWE studies are performed at high latitudes (EISCAT and HAARP) whereas TID excitation are better performed at mid-latitudes. The different magnetic field inclination at the various locations has important effects on the generation of the plasma instabilities. This offers opportunities to perform complementary studies of wave-plasma interactions.

\subsection{Satellites}

Satellites are important for many active experiments conducted in space. In many cases satellites have their own scientific program and their participation in active experiments consist of collecting in-situ wave and particle data in the regions where these waves and particles are 
injected/generated by other means. These data are collected only when the satellite occurs "in the right place at the right time", because the orbit and ephemeris of the satellite cannot be changed and thus active experiments must be conducted when the satellites are in a vicinity of the heating facility or its magnetically conjugate location.

There are many interesting and succesfull active experiments including satellites and ground facilities reported in the literature. For example, NASA's FAST satellite detected ULF waves injected into the magnetosphere in the experiments with heating the auroral electrojet [Robinson et al., 2000; Kolesnikova et al., 2002]. CASSIOPE/e-POP satellite has been used to receive HF waves from the SURA and EISCAT heating facilities, with one aim of investigating the ionospheric "radio window" where $\mathrm{O}$ to $\mathrm{Z}$-mode conversion in the $\mathrm{F}$ region occurs [James et al., 2017]. Transmissions from the VHF-UHF beacon Coherent Electromagnetic Radio Tomography (CERTO) on CASSIOPE can be used together with the measurements from the ground recivers to reconstruct a two-dimensional distribution of electron plasma frequency in the ionospheric $\mathrm{F}$ region [Siefring et al., 2014].

The most recent trend showing great promises to enhance the science return from active space experiments is a development of specialized CubeSats. Because of their low cost (they are frequently built from commercial-off-the-shelf components), small size $(\sim 10 \mathrm{~s} \mathrm{~cm})$ and light weight (few $\mathrm{kg}$ ), CubeSats can be produced and launched in larger quantities than conventional satellites allowing multipoint observations with constellations of satellites. To date, hundreds CubeSats have been launched into orbit as secondary or tertiary payloads on larger missions.

A successful example of the application of CubeSats to study the properties of the ionosphere, is the Dynamic Ionosphere CubeSat Experiment (DICE) mission designed mainly at the Space Dynamics Laboratory, Utah State University [Fish et al., 2014]. It focuses on the investigation of physical processes responsible for formation and evolution of the Storm Enhanced Density bulge and plume in the noon to post-noon sector during magnetic storms in the mid-latitude ionosphere over North America.

Another example is the NSF sponsored Radio Aurora Explorer (RAX-2) satellite. This threeunit (3U) CubeSat built by the University of Michigan and the Stanford Research Institute was used in conjunction with the Poker Flat Incoherent Scatter Radar (PFISR) to measure radar scatter at orbital altitudes from the ionospheric irregularities [Bahcivan et al., 2014].

A constellation of CubeSats launched on a single rocket and deployed at the same time provides multipoint sampling or effectively extends the aperture of a science mission. Several CubeSats launched into the same lead/trail orbit in a so-called "pearls-on-a-string" configuration can sample an ionospheric region heated from the ground and help separate spatial and temporal variations. Similarly, a group of CubeSats flying in formation abreast can simultaneously sample regions within and external to a heated region. The Spire Global Inc. has launched constellations of CubeSats in low and high inclination orbits with GPS radio occultation payloads and plans to operate dozens of CubeSats for global monitoring of ionospheric electron density and lower 
atmosphere applications. Several other examples of using CubeSats to study local ionospheric inhomogeneities over the heating facilities are given by Chernyshov et al. [2016].

More examples of active experiments involving ground recievers/transmitters and satellites will be given in section 4. In this part of the review we will discuss in more detail four particular "past, present and future" satellite missions actively involved in active experiments. We start with one of the most succesful satellite project involving observations of waves and particles above HAARP and SURA heating facilities - the Detection of Electro-Magnetic Emissions Transmitted from Earthquake Regions (DEMETER) mission.

\subsubsection{DEMETER Satellite}

The primary objective of the DEMETER mission was to study disturbances in the plasma, waves or energetic particle populations that might occur prior to the earthquakes in the ionosphere close to epicenter. Designed and built by the Toulouse Space Center as the first micro-satellite of the CNES MYRIAD program, DEMETER was launched on the planned orbit from Baïkonour on June 28, 2004 by a DNEPR rocket [Cussac et al., 2006]. With a mass of $130 \mathrm{~kg}$ and a total power consumption of $\sim 50 \mathrm{~W}$, the satellite was equipped with a single solar panel deployed from one side and nearly perpendicular to orbit plane. To cope with the required high sensitivity of plasma and wave measurements, considerable efforts were made to minimize interferences and stray electric fields from the spacecraft and sub-systems. $85 \%$ of its external surface is thus covered by a carbon filled conductive MLI at ground making the entire spacecraft surface as close as possible to equipotential. The electromagnetic or electrostatic noise radiated by the solar cells are, for the greatest part, shielded by coating the sunlit face of each cell with a grounded, transparent, thin conductive layer of stain oxide Figure 2.5 displays a view of DEMETER in its in-flight configuration with the solar panel and all booms deployed and Figure 2.6 exemplifies the longitude displacement in successive orbits.

The anticipated weak disturbances require that accurate base-lines of the measured parameters in absence of seismic activity be known. Both the ionosphere and the electromagnetic waves are affected by large day-to-day variations including quite regular daily and seasonal effects due to the varying solar illumination and more irregular, large amplitude variations driven by auroral activity or atmospheric events, such as atmospheric gravity waves. The second objective assigned to DEMETER, was thus "space weather oriented" and aimed at

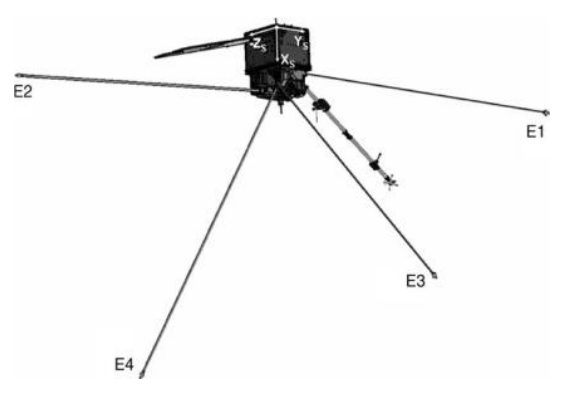

Figure 2.5. DEMETER in-flight configuration. studying the natural ionospheric disturbances over periods with no seismic activity.

Finally, significant effects in the ionosphere such as ELF/VLF power lines and scattering of energetic electrons from radiation belts by VLF transmitters and the strong ionospheric disturbances by high-power HF facilities also result from man-made activities. This last set of phenomena constituted the third objective assigned to the DEMETER mission. 
To achieve these scientific objectives, the DEMETER scientific payload, described extensively in a dedicated issue of Planetary and Space Science (Volume 54, Issue 5, 2006), consists of a set of 5 instruments. Two of them, ISL and IAP, measured the electron and ion components of the thermal plasma, IDP detected energetic electrons and the last two, ICE and IMSC, were respectively devoted to measurements of $\mathrm{DC}$ and $\mathrm{AC}$ electric fields and $\mathrm{AC}$ magnetic fields. In addition, a magnetometer used for attitude control before orbit injection was also operated simultaneous with the scientific instruments, providing low resolution measurements of the Earth's magnetic field.

The required high sensitivity measurements of this whole set of parameters are better achieved by performing observations as close as possible to the source region, thus at low altitude. To minimize the daily variations along the orbit and reduce the statistical uncertainties of the reference base-lines of all measured parameters, an orbit at a constant local time provides the best choice since, over a given region on Earth, the effects linked to a variable solar illumination are practically eliminated. Altogether, these considerations led to select a quasi-sun-synchronous orbit with a $98^{\circ}$ inclination, an ascending node in the early night sector at $\sim 22.30 \mathrm{LT}$ and an altitude of $715 \mathrm{~km}$ at launch, a set of orbital parameters that are very close to those of most Earth observation satellites. Two years after launch, the altitude was lowered to $650 \mathrm{~km}$ so that atmospheric braking will lead to a re-entry and loss of the satellite after a maximum of 25 years in orbit as required by international regulations.

During the entire operational life-time of the satellite, till December 9, 2010, nearly continuous operations were

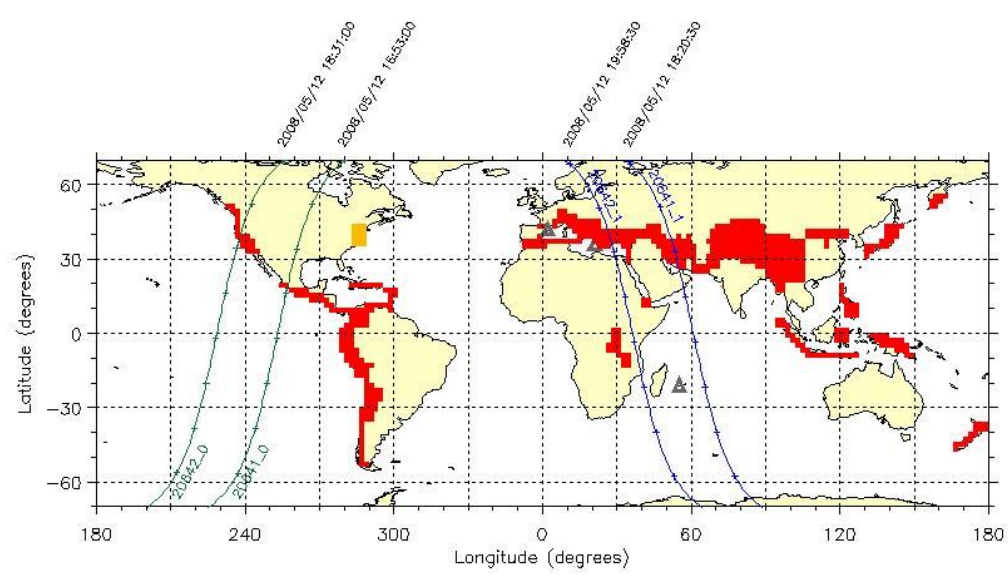

Figure 2.6. Two successive DEMETER orbits: the two dayside downgoing half-orbits are shown in green, the two night-side up-going half orbits are shown in blue. The ascending and descending nodes move by $\sim 24^{\circ}$ westward from one orbit to the next.

achieved on both day and night

half-orbits at latitudes less than $60^{\circ}$ where most of the active seismic zones are located. In addition, specific measurement sequences were programmed at higher latitudes mainly associated with the operation of ground-based facilities such as EISCAT and HAARP. During payload operation, data from scientific instruments and onboard sub-systems are stored in memory. Two times a day, when the satellite flies over a CNES TM station, the memorized data are sent through TM to the DEMETER Data Center and processed.

DEMETER has two modes of operation: Survey and Burst. Burst modes provide high resolution measurements and are programmed regularly over all seismic regions and, during specific sequences, when above active ground-based facilities. Survey modes are operated during 
the remaining intervals of time to get lower time resolution measurements over larger orbit paths which help building parameters reference base-lines and achieve space-weather objectives.

During the six years of DEMETER operational phase, from 2005 to 2010, a number of joint observations were coordinated with the SURA, HAARP and EISCAT high power HF transmitters. The main objectives of these combined experiments fall in two categories, in-situ measurements of plasma and wave disturbances or detection of ELF and VLF wave emissions triggered by the interaction of the HF waves with the ionosphere.

An extensive program was performed with the SURA heating facility. There were $~ 200$ satellite passes over SURA during the 6 years of DEMETER operations. The main goal of this program was to study the formation, structure and characteristics of ducts in the heated ionosphere and their role in ionosphere-magnetosphere coupling and VLF wave propagation. An overview of the results obtained during the DEMETER joint program was given by Frolov et al., [2016] and references therein.

During the DEMETER joint experiments O-mode HF waves were radiated either towards the zenith (antennas at $0^{\circ}$ elevation) or at an elevation of $12^{\circ}$ South to benefit from the "magnetic zenith effect" [Gurevich, 2007]. In such a configuration, the HF waves are refracted during their travel through the lower ionosphere so that their propagation vector is $\sim$ parallel to the Earth's magnetic field at their reflection altitude which maximizes the ionospheric disturbances along the corresponding flux tube. The SURA facility was switched on for 10 to 15 minutes before the satellite reached closest distance from the center of the heated magnetic flux tube. This was shown by Vas 'kov et al. [1998], Gladevich et al. [2003] and Frolov et al. [2007] to be sufficient for the development of ionospheric disturbances to a stationary level over the full range of altitudes from the pump-wave reflection altitude in the bottom-side F-region to $\sim 800 \mathrm{~km}$, about $100-150 \mathrm{~km}$ above the DEMETER orbit and at the altitude of the DMSP satellites from which complementary plasma data were obtained in some cases. The heating time was increased to 40 minutes in a few (unsuccessful) cases in 2010 when it was attempted to search for detectable disturbances in the conjugate ionosphere.

Finally, IDP measurements of precipitating energetic electrons provide interesting observations. They demonstrated enhanced electron precipitations in the low energy band $(70<\mathrm{E}$ $<150 \mathrm{keV}$ ), which according to the general discussion of IDP observations in Sauvaud et al., [2006] is due to the scattering of radiation belt electrons by a VLF transmitter operating in this longitude zone.

\subsubsection{Defense Meteorological Satellite Program (DMSP)}

The Defense Meteorological Satellite Program (DMSP) is the longest, more than 50 years, running production satellite program ever. The DMSP spacecraft monitor meteorological, oceanographic, and solar-terrestrial physics for the United States Department of Defense. The last, DMSP 19, satellite was launched on April 3, 2014. Currently, three satellites F16, F17, and F18 are collecting data (F19 is considered lost as of July 2016). Each of the DMSP spacecraft is a three- 
axis stabilized satellite flying in circular, sun-synchronous polar (inclination $98.7^{\circ}$ ) orbit at an altitude of $\sim 840-850 \mathrm{~km}$ (see Figure 2.7). The geographic local times of the orbits are either near the 1800-0600 or 2100-0900 meridians. Due to the offset between the geographic and geomagnetic poles DMSP satellites sample a wide range of magnetic local times (MLT) over the course of a day. The ascending nodes of DMSP orbits are on the dusk side of the Earth. Thus, the satellites move toward the northwest in the evening LT sector. Besides meteorological and oceanographic sensors, each satellite carries a sophisticated sensor suite to measure fluxes of auroral particles (SSJ4/5), the densities, temperatures, and drift motions of ionospheric ions and electrons (SSIES), and after 1995 perturbations of the Earth magnetic field (SSM).

Identical SSJ4/5 sensors are mounted on the top sides of DMSP satellites to measure fluxes of precipitating electrons and ions in the energy range between $30 \mathrm{eV}$ and $30 \mathrm{keV}$. The measurements are made by 4 detectors, one high energy detector and one low energy detector for each of the particle types. The ion detectors have no mass discrimination capabilities. Each detector has 10 logarithmically spaced energy steps. The high energy detectors step from $30 \mathrm{keV}$ to $1 \mathrm{keV}$ and the low energy detectors step from $1 \mathrm{keV}$ to $30 \mathrm{eV}$. Only particles within an

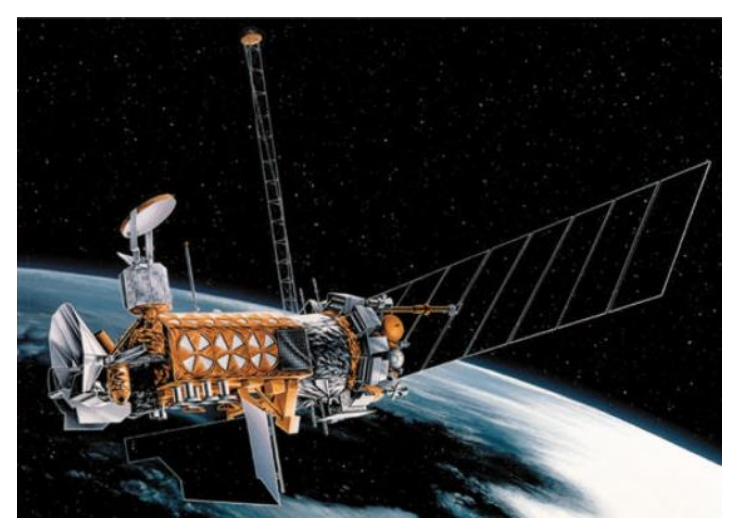

Figure 2.7. The artist's view of a DMSP satellite. energy band of approximately $10 \%$ of the channel step energy freely pass from aperture to the detector. The particle fluxes are measured within a solid angle of $2^{\circ}$ by $5^{\circ}$ for the high energy channels and $4^{\circ}$ by $5^{\circ}$ for the low energy channels centered on local vertical. Each detector has a dwell time of $0.098 \mathrm{sec}$ and a $0.002 \mathrm{sec}$ period between steps to stabilize the voltage. Each detector makes a complete 10 step sequence in 1 second. One 20-point ion and one 20-point electron sequence is returned once per second.

SSIES sensors are mounted on the ram facing surfaces of the satellites. They consist of an ion drift meter to measure the horizontal and vertical cross-track components of plasma drift within the range of $\pm 3000 \mathrm{~m} / \mathrm{s}$ and a one-bit resolution of $12 \mathrm{~m} / \mathrm{s}$ for ambient ion densities greater than $5 \cdot 10^{3} \mathrm{~cm}^{-3}$, retarding potential analyzer to measure ion temperatures, composition, and the in-track component of plasma drift, an ion trap to measure the total ion density, and a spherical Langmuir probe mounted on an $80-\mathrm{cm}$ boom to measure the density and temperature of ambient electrons [Rich and Hairston, 1984]. The drift and density measurements are sampled at 6 and $24 \mathrm{~Hz}$, respectively. It takes 4 seconds to sample temperatures.

SSM sensors are tri-axial fluxgate magnetometers mounted on the bodies of the F12--F14 satellites and since DMSP F15, on 5-m booms to reduce spacecraft-generated electromagnetic contamination. Magnetic field components are sampled at a rate of 12 (Y and Z) and $10(\mathrm{X}) \mathrm{s}^{-1}$ in a satellite-centered coordinate system. The $\mathrm{X}$ axis points in the downward direction. The $\mathrm{Y}$ axis points along the spacecraft velocity. The $\mathrm{Z}$ axis completes a right-hand coordinate system. Positive 
$\mathrm{Z}$ components generally point in the anti-sunward direction. Data are presented as differences $(\Delta \mathrm{B})$ between measured values and those assigned by the IGRF-90 magnetic field model.

In the past decade, various DMSP satellites have been used in conjunction with the SURA, EISCAT, and HAARP heating experiments to measure artificial density ducts and ion outflows in the topside ionosphere [e.g., Frolov et al., 2016; Milikh et al., 2008a; 2010a; Blagoveshchenskaya et al., 2011].

\subsubsection{The Demonstration and Science Experiments (DSX) Satellite}

The Air Force Research Laboratory has developed the Demonstration and Science Experiments (DSX) to investigate 1) very-low-frequency electromagnetic wave-particle interactions (WPIx) in medium-earth orbit (MEO) region of space between the Van Allen radiation belts, the "slot" region; 2) space weather effects in the slot region (SWx); and 3) space environmental effects (SFx) on spacecraft components in the slot region [Fennelly, 2009; Scherbarth et al., 2009]. In addition to the DSX spacecraft, VLF and Particle Mapper (VPM) nanosatellite will be launched to perform far-field measurements of the in situ transmitter [Gies et $a l ., 2014]$. The DSX mission is planned to be launched in 2018 aboard SpaceX Falcon Heavy for a nominal one year mission. It will fly in a $6000 \times 12000 \mathrm{~km}$ elliptical $\left(42^{\circ}\right.$-inclination) orbit covering the outer region of the inner radiation belt, the slot region, and the inner region of the outer radiation belt in a 5.3 hour period. The planned initial orbit has apogee and perigee near the equator, with an orbit precession period just over one year. Figure 2.8 shows a schematic view of the DSX space flight experiment with the VPM nanosat.

The SFx module [Scherbarth et al., 2009] consists of the NASA Space Environment Testbeds-1 (SET-1) and radiometers and photometers provided by the Air Force Research Laboratory Aerospace Systems Directorate. The objectives of SET-1 are to improve engineering approaches to accommodate and/or mitigate the effect of solar variability on spacecraft design and operations, reduce risk for new technologies infused into future space missions, and provide a standard mechanical, electrical, and thermal interface for a collection of small flight investigations. The SET-1 payload consists of two units, the Correlative Environment Monitor (CEM) and the Central Carrier Assembly (CCA). The carrier provides a single interface for power and data between the

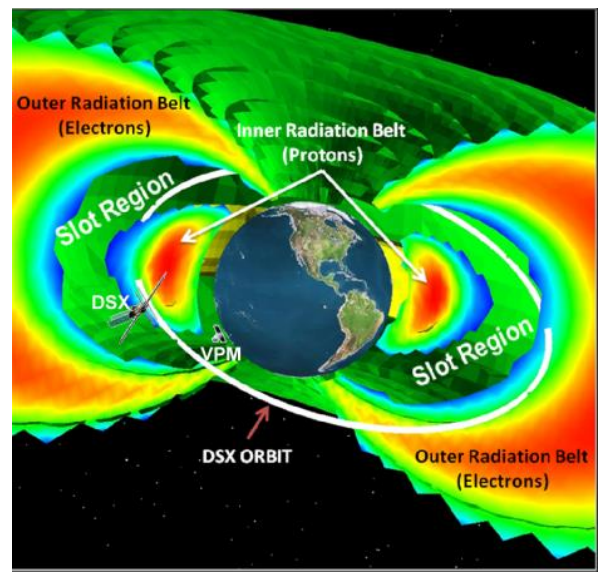

Figure 2.8. An illustration of the DSX space flight experiment with the VPM nanosatellite [Fennelly, 2009]. DSX spacecraft and the SET-1 microelectronic investigations (inside the CCA) and CEM.

The WPIx module [Scherbarth et al., 2009] is the one relevant to the topic of this review, so it will be described in more details. The module contains a VLF transmitter, broadband (BBR) and narrowband (NBR) receivers, tri-axial search coil (TASC) and DC vector (VMAG) magnetometers, and loss-cone imager (LCI). The BBR and TASC along with Y and Z linear, orthogonal dipole antennas with two electric components make up the VLF broadband receiver, with the frequency range $0.1-50 \mathrm{kHz}$ and the sensitivity $10^{-16} \mathrm{~V}^{2} / \mathrm{m}^{2} / \mathrm{Hz}$ and $10^{-11} \mathrm{nT}^{2} / \mathrm{Hz}$. The 
receiver was built by Stanford University, NASA/Goddard, Lockheed Martin, and ATK Space Systems. The NBR and Y antenna constitute the VLF narrowband receiver covering the band from $3 \mathrm{kHz}$ to $750 \mathrm{kHz}$. The LCI consists of a High Sensitivity Telescope (HST) for measuring $100-$ $500 \mathrm{keV}$ electrons with $0.1 \mathrm{~cm}^{2}$-str geometric factor within $6.5^{\circ}$ loss cone, and a Fixed Sensor Head (FSH) for $50-700 \mathrm{keV}$ electrons with $130^{\circ} \times 10^{\circ}$ pitch angle distribution. The LCI instrument was built by Boston University. Finally, the VMAG instrument is capable of $0-8 \mathrm{~Hz}$ three axis measurements of magnetic field line measurements at +/- $0.1 \mathrm{nT}$ accuracy. The VMAG was built by the University of California, Los Angeles (UCLA).

The VLF transmitter was built by the University of Massachusetts Lowell (UML), Southwest Research Institute, and ATK. The transmitter will operate in high power at $2-50 \mathrm{kHz}$ at the $\mathrm{kV}$ level for up to 30 min per orbit occurring near the magnetic equator $\left(|\mathrm{MLAT}|<20^{\circ}\right.$ or $\left.\mathrm{L}<3.5\right)$ and also will coordinate with conjugate target teams. An additional "sounding" low power mode at 50$750 \mathrm{kHz}$ will also be used for plasma characterization during the mission.

The $\mathrm{Z}$ antenna (16 $\mathrm{m}$ tip-to-tip) functions as a VLF receive antenna in a cross-dipole configuration with the Y antenna. The TASC and VMAG instruments are placed at opposite tips of the $\mathrm{Z}$ antenna (16 $\mathrm{m}$ tip-to-tip) to separate them from the rest of the DSX instruments which would interfere with their operation as VMAG and TASC measure the local DC and AC magnetic fields, respectively. The $\mathrm{Y}$ antenna ( $80 \mathrm{~m}$ tip-to-tip) functions as a VLF receive and transmit antenna. Their booms are both built by ATK Space Systems.

The $\mathrm{Y}$ antenna boom is a truss consisting of Graphite-Epoxy $(\mathrm{Gr} / \mathrm{Ep})$ longerons and batten elements with steel diagonals. In order to perform the VLF antenna function, copper wire is run

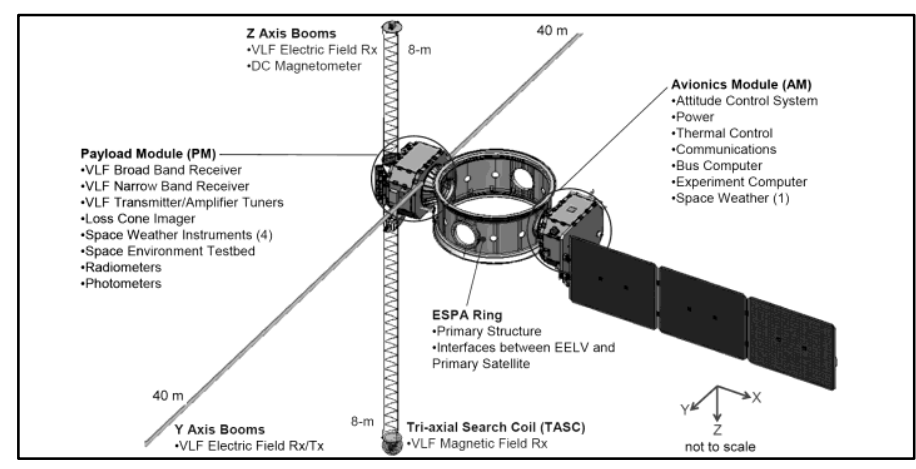

Figure 2.9. DSX baseline deployed configuration. the full length of each truss's three longerons, attached at every other joint. The $\mathrm{Z}$ antenna boom is a similar truss with S-2 glass (fiberglass) material for the longerons and battens instead of the $\mathrm{Gr} / \mathrm{Ep}$. Both booms use frangibolt systems to constrain them within canisters through launch. Once on-orbit, the spacecraft powers the frangibolts in order to heat their Nickel-Titanium (NiTi) collars to the point that they break their bolts and release the tip plates from the canisters. The longerons are continuous elements that are "spring loaded" into the canisters via coiling. Thus, once released, the stored strain energy of each coiled system deploys the structures into their minimally strained, full length trusses. The deployment rate of each truss system is controlled by a lanyard with a geared friction, keeping the trusses from damaging themselves with excessive accelerations and/or sudden decelerations.

The transmitter design is based on NASA's Imager for Magnetopause-to-Aurora Global Exploration (IMAGE) Radio Plasma Imager (RPI) instrument [Reinisch et al., 2001] that operated at $3 \mathrm{kV}$ and was optimized for $>50 \mathrm{kHz}$. The DSX design optimizes the transmitter impedance dependent on frequency, antenna length, and diameter. DSX is flying the first ever VLF "dynamic tuning" technology to adjust circuit parameters in real time. The voltages are limited to $<10 \mathrm{kV}$ 
due to critical component limits. The DSX system is nominally designed for $5 \mathrm{kV}$ with the capability to go to $10 \mathrm{kV}$ at the end of life.

Figure 2.9 shows the functional baseline configuration for the DSX flight experiment. The core is the Evolved Expendable Launch Vehicle (EELV) Secondary Payload Adapter (ESPA) ring, which is used to maximize launch opportunities. The ESPA ring comprises the primary structure for DSX, and is upgraded to provide host spacecraft functions (e.g., avionics and power management and distribution) by the addition of components packaged on an avionics module (AM). The DSX payloads (including deployable booms) are mounted on an identical structure, the payload module (PM), attached to the ESPA ring opposite the avionics module. The AM and PM together comprise the DSX Host Spacecraft Bus (HSB). The entire assembly is designed to be stowed within a 4-m diameter EELV fairing.

The VPM nanosat is developed in AFRL/RV to quantify space and terrestrial VLF injection and resulting particle precipitation. The VPM $6 \mathrm{U}, 10 \mathrm{~kg}$ spacecraft will be launched from ISS in a circular orbit of $400 \mathrm{~km}$ with $51^{\circ}$ inclination for 1 year minimum mission. Its payload consists of dual channel VLF receiver, loss cone and trapped electron spectrometers, AC magnetic search coil, deployable E-field antennae, and B-field boom.

\subsubsection{RESONANCE Satellite}

The international space project RESONANCE [Mogilevsky et al., 2012, Demekhov et al., 2003] is planned with the participation of scientific teams from Russia, Ukraine, Austria, Bulgaria, Germany, Greece, Poland, Slovakia, the USA, Czech Republic, Finland and France and aims to study the resonance interaction of waves and particles in the inner magnetosphere of the Earth. The Earth's inner magnetosphere is an important link in a long chain of solar-terrestrial relations. Hot magnetospheric plasma, cold plasmaspheric particles and, in contrast, high energy charged particles of the Earth's radiation belts are found together in the inner magnetosphere. Such nonequilibrium state of plasma is connected with the generation of various plasma oscillations actively interacting with particles which leads both to spatial diffusion and diffusion in a velocity space. In fact, the latter influences particle precipitation through pitch-angle diffusion and their lifetime in the Earth's magnetosphere. Thus, one of the most important problems of near-Earth studies is the nature of the interconnection of micro-, meso- and macro-scale processes, especially in the active layers of the upper atmosphere. At the same time, smallest-scale phenomena are most difficult to study experimentally since ones requires a careful coordination of space vehicles with measuring instruments in space and time. The project RESONANCE is aimed to study the whole complex of these issues.

A unique part of this project will be a joint experiment with a ground-based facility of radiofrequency heating of the ionosphere, which will study of ionospheric physics and test the

possibility of controlling some natural powerful processes in the near-Earth plasma.

The choice of the satellite orbits is thus of high importance. One of the most interesting options is the possibility of organizing measurements in the vicinity of a specially selected magnetic field line, since particle and energy exchange between the ionosphere and the magnetosphere mainly 
occur within a tube of force of the Earth's magnetic field. These processes include in particular: the propagation of whistler-mode and Alfvén waves and the interaction with these waves plays a key role in the electron and ion dynamics in the radiation belts; the precipitation of energetic particles into the ionosphere; the filling of magnetic flux tubes with cold and suprathermal plasma; the motion of energetic particles from the regions of auroral acceleration and regions of magnetic reconnection. To solve these problems requires simultaneous multipoint measurements of plasma parameters and electromagnetic radiation in the selected flux tubes of the magnetic field.

One of the main features of the RESONANCE project is the choice of the novel type of a magneto-synchronous orbits proposed and designed for this project that ensure the presence of four satellites in different places of the same magnetic flux tube during a long period of time (relatively to the typical time-scale of magnetospheric processes). The new planned orbital set will consist of two pairs of spacecraft, R1A, R1B and R2A, R2B, each pair on its own orbit. The parameters of these orbits are as follows: orbital period is $8 \mathrm{hrs} 15 \mathrm{~min}$, apogee $\sim 27341 \mathrm{~km}$, perigee $\sim 500$ $\mathrm{km}$, inclination is $63.4^{\circ}$, and the co-rotation time in a magnetic flux tube at $\mathrm{L} \sim 4-5$ is up to 3 hours if the transverse scale of the flux tube is about $100 \mathrm{~km}$ at the ionosphere. The spacecraft within

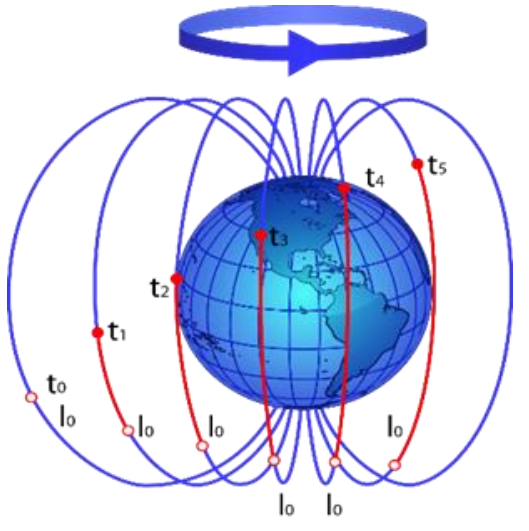

Figure 2.10. RESONANCE magneto-synchronous orbit: a view from an inertial reference frame. a pair will be separated by several hundred kilometers on average. An important constraint is the magnetic latitude of the orbit apogee, which must be in the inner magnetosphere not far from the plasmapause, otherwise reliable co-rotation is impossible. For active experiments the flux tube should map to the heating facility. According to theory and experiment, a few hundred kilometers is the characteristic scale on which chorus generation is developed. This is the reason why the time during which two satellites remain within this distance is important.

On the other hand, larger scales and effects of wave propagation far from the generation region are also of interest. That is why another pair of spacecraft located sufficiently far from the first one should be useful for the investigation of multiple scale phenomena in wave-particle interactions. A view of magneto-synchronous orbit from an inertial reference frame is shown in Figure 2.10. It should be noted that the apogees and perigees of these two orbits are located in different hemispheres. Two satellites in each pair move along the same orbit, although the distance between satellites vary and is controlled by a telemetric system. It is worth mentioning that a part of each orbit lies in the sub-auroral region of the magnetosphere where important processes which control geomagnetic storms take place. Moreover, the relative position of the two spacecraft pairs is such that one is in the auroral zone when the other is in the equatorial region. As a satellite moves along its orbit, the magnetic flux tube rotates together with the Earth. With an appropriate choice of the orbit parameters, the satellite remains in the same flux tube for an extended period of time. Thus, Figure 2.10 demonstrates the motion of a RESONANCE satellite along the magnetic flux tube, where $1_{0}$ is the initial position of the satellite in the flux tube (at time $t_{0}$ ), $1_{i}$ is the satellite position 
at time $t_{\mathrm{i}}$. Bold red lines show the distance covered during that time. Spacecraft trajectories in a meridian plane rotating with the Earth are shown in Figure 2.11.

In order to determine the localization of the interaction region and the size of the radiation source, the other two orbits will be used. In these orbits, the satellites are on different field lines, and thus two possible options are chosen. In the first variant, the first of the satellites will be on a field line closer to the Earth than the magnetic field line on which the second satellite will be located. However, both satellites will measure at the same MLT. In this variant, the radial dimensions of the interaction region will be determined. In the second variant, the satellites are at equal distances from the Earth but at different MLT. Hence, such a configuration of satellites makes it possible to determine the meridional dimensions of the interaction region.

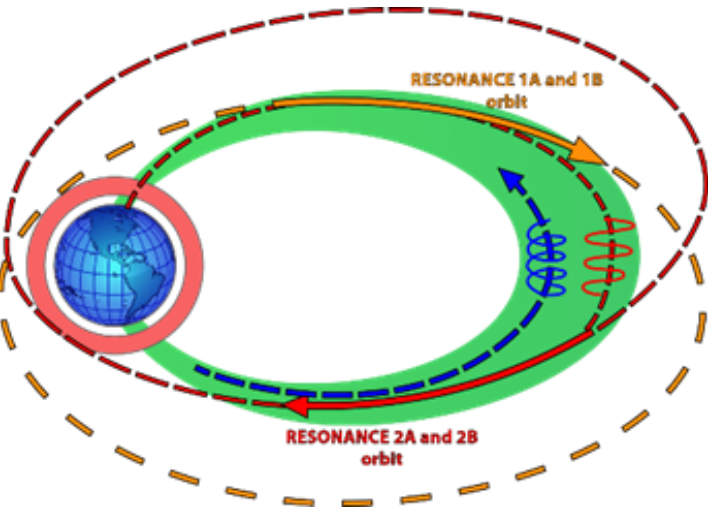

Figure 2.11. Orbits of two satellites in the Earth's reference frame. Two pairs of satellites (1A, 1B) and $(2 \mathrm{~A}, 2 \mathrm{~B})$ will be launched on two different orbits, each pair on the same orbit. The distance between satellites of one pair can be managed by space control.

Four RESONANCE satellites are to be launched to study the outer zone of the Earth's radiation belt where, in particular, geostationary satellites operate. The project will study the so-called relativistic electrons - the main component of the radiation belts, which are the main hazardous factor to communication satellites in geostationary orbits. RESONANCE orbiters will study with high temporal resolution the processes of electron acceleration after interaction with electromagnetic waves.

The instruments onboard of RESONANCE satellites include: 1) DC and AC magnetometers; 2) DC/ULF fields analyzer (0-35 Hz, dynamic range: DC - $120 \mathrm{~dB}$, ULF - $80 \mathrm{~dB})$; 3) ELF/VLF fields analyzer (3 electric and 3 magnetic components, 0.01-30 kHz, dynamic range: $70 \mathrm{~dB}$ ); 4) $\mathrm{HF}$ fields analyzer (3 electric and 3 magnetic components, 0.01-30 MHz, dynamic range: $70 \mathrm{~dB}$ ); 5) Mutual impedance probe for plasma density and temperature measurements; 6) Thermal plasma spectrometer: electrons and $\mathrm{H}^{+}, \mathrm{He}^{+}, \mathrm{O}^{+}$ions with energies 1-100 eV and time resolution 1-5 sec; 7) Hot plasma spectrometer: electrons and 3 sorts of ions, with energies 10-104 eV and time resolution $1 \mathrm{sec}$; 8) Fast electron spectrometer (5-50 keV, energy resolution $100 \mathrm{eV}$, time resolution $10 \mathrm{~ms}$ ); 9) Energetic particle instrument; 10) Radio interferometer. Satellite locations will be determined with the onboard GPS/GLONASS navigation receivers.

The scientific program of the project consists of two parts. The first, "passive" part, is aimed at the study of natural magnetospheric phenomena. Main goals of this part are as follows:

- Magnetospheric cyclotron maser and its long-term evolution.

- Role of small-scale phenomena in the global dynamics of magnetospheric plasma.

- $\quad$ Ring current and outer radiation belt formation and evolution, $\mathrm{MeV}$ electron dynamics.

- Plasmasphere dynamics and refilling, sub-auroral zone physics. 
- Plasma injection development, magnetic field reconfigurations.

- Mid-altitude auroral zone, polar cap and cusp physics.

- Generation mechanism, fine structure, and propagation of the Auroral Kilometric Radiation (AKR).

Simultaneous satellite and ground-based measurements will also allow to carry out original studies in the magnetosphere of ULF/VLF fields transmitted through ionospheric resonance systems, namely, the Schumann and ionospheric Alfvén resonators.

Investigations in the frame of the second, "active" part will focus on joint experiments of the RESONANCE satellite(s) with the ground-based HF heating facilities (e.g. HAARP). We expect that parameters of the natural magnetospheric oscillatory system will change, if powerful $\mathrm{HF}$ electromagnetic emissions heat the ionosphere and thus modulate the ionospheric mirrors. Phase and amplitude of magnetospheric oscillations, measured onboard the RESONANCE satellite, will be transmitted to the heating facility and used to modulate the HF radiation. In the case of in-phase modification, the amplitude of the natural oscillations should increase, whereas inverse anti-phase modification should decrease the oscillation. Such a unique experiment will help to investigate important underlying principles in cyclotron maser theory and clarify the role of ionospheric mirrors in wave generation.

Other interesting phenomena which could be studied by the RESONACE satellites are repeated periodic bursts of the scattered probe signal intensity during the field-aligned irregularities relaxation stage of the disturbed region after the pump switches off. These periodic bursts were first observed in 1987, and the phenomenon was named "echo scattering" or "reverberative scattering". This effect was explained by the generation of an Alfvénic pulse within the disturbed region during the heating facility switch off. This pulse propagates along the magnetic field line and reflects back at the conjugate point in the opposite hemisphere and then regenerates the smallscale irregularities. Coming back to the region filled with decaying irregularities, the pulse "pumps" them, which is evident in the scatter signal intensity. Note that observations of the echo scattering at the high-latitude EISCAT facility show a time delay of about $200 \mathrm{~s}$ after pump off, which is much longer than at SURA (approximately $30 \mathrm{~s}$ ), consistent with the longer bounce period for the Alfvén pulse in the longer magnetic flux tube at the higher magnetic latitude of EISCAT [Blagoveshchenskaya et al., 2006, Leyser and Wong, 2009]. In addition, an increase in the intensity of artificial ionospheric inhomogeneities in the field of a natural MHD wave was recorded in various measuring sessions [Ponomarenko et al., 2000] indicating a nonlinear interaction of MHD fields with small-scale inhomogeneities in the ionospheric plasma.

The effect of "echo-scattering" can be studied in detail in the framework of the RESONANCE project. While the satellite constellation is located above the HAARP facility, it will be possible to investigate the mode composition of artificially excited MHD waves and to measure the velocity and direction of their propagation. Furthermore, a heating facility is a deterministic in time and space excitation source of MHD waves which can be used for testing and calibration of algorithms for evaluation of MHD wave parameters from the signals measured on the satellites. Note the 
calculated orbital period of the spacecraft is 8 hours. This means that the spacecraft will make three revolutions during one day, in other words, the spacecraft will be located once per day in the selected magnetic flux tube connected to the HAARP through active period.

The goals of RESONANCE-HAARP joint experiments include studies of: 1) artificial excitation and/or stimulation of various electromagnetic wave-modes, in particular, ELF/VLF and ULF waves excited by the ionospheric heating; 2) magnetospheric injection and Artificially Stimulated Emissions (ASE); 3) modification of precipitation particle fluxes caused by nonlinear interactions between excited waves and energetic charged particles; 4) electron acceleration by the Langmuir turbulence caused by HF heating; 5) variation of magnetospheric maser Q-parameter (quality factor) by modification of the reflection coefficient from the ionosphere in selected magnetic flux tube, and, possibly, formation of artificial ducts; 6) upper hybrid waves and conversion of lower hybrid waves to whistlers. 


\section{Theory of the HF Ionospheric Modification}

Interaction of HF pump waves with the ionosphere is mediated by the excited plasma turbulence. When a radio wave with an ordinary polarization (O-mode) approaches the reflection altitude, $h_{0}$, its frequency, $f_{0}=\omega_{0} / 2 \pi$, is close to the local electron plasma frequency, $f_{p e} \approx$ $9 \sqrt{n} \mathrm{kHz}$ ( $n$ is the plasma density in $\mathrm{cm}^{-3}$ ). In other words, it is the plasma resonance region where the radio pump wave can couple to the plasma eigenmodes near $f_{p e}$, notably, Langmuir waves. Matching $f_{0}$ to the upper hybrid (UH) resonance frequency, $f_{u h r}=\sqrt{f_{p e}^{2}+f_{c e}^{2}}$, defines the UH resonance altitude $h_{u h} \approx h_{0}-L_{n} \frac{\omega_{c e}^{2}}{\omega_{0}^{2}}$. Here $L_{n}=\left|d \ln n_{e} / d h\right|^{-1}$ is the plasma density scale height, $f_{c e}$ is the electron cyclotron frequency. Accordingly, the plasma and upper hybrid resonance layers are the regions where O-mode radio waves interact with the ionosphere most efficiently.

The variety of natural plasma eigenmodes results in a great number of nonlinear phenomena driven by the resonant action of high-power pump waves in the two layers. Particularly, Langmuir turbulence (LT) near $h_{0}$ is manifested by the enhanced plasma line (PL) and ion line (IL) in the incoherent radar backscatter (section 4.6). Incoherent scatter radars provide one of the best diagnostics to study the plasma waves excited by the HF pump [e.g., Rietveld et al., 2000]. A detailed discussion of the state of Langmuir turbulence experiments and theory from a radar perspective is given by Djuth and DuBois [2015]. Even though the authors focus on the Arecibo $430 \mathrm{MHz}$ radar observations of waves far from field-aligned, a comparison is made with the EISCAT 933 and $224 \mathrm{MHz}$ radars at or close to field-aligned.

In turn, UH processes near $h_{u h}$ create magnetic field-aligned density irregularities (FAIs) that cause enhanced HF radar backscatter (artificial radio aurora) and the anomalous absorption of radio signals (section 4.2). Merging of different plasma modes produces secondary or stimulated electromagnetic emissions (SEE) (section 4.1). Heating and acceleration of plasma particles by the excited waves result in $\mathrm{O}^{+}$ion upflows and density ducts in the topside ionosphere (section 4.3), enhanced optical emissions dubbed artificial aurora (section 4.4) and artificial ionization layers (section 4.6). Optical emissions with the excitation energies $>2-18 \mathrm{eV}$ indicate suprathermal, $\varepsilon>>T_{e}$, electrons (section 4.4) that are also manifested by the enhanced plasma lines far from the heated region [Carlson et al., 1982; 2017].

Several basic features of plasma turbulence and artificial aurora have been established at a relatively low effective radiated power (EPR) $P_{0}$ from $10 \mathrm{~s}$ MW to $100 \mathrm{MW}$ (ERP $=$ transmitter output $\times$ antenna gain). First, the magnetic zenith (MZ) effect, i.e., stronger artificial aurora, electron heating, and anomalous absorption (see sections 4.2.7 and 4.4.3) and the faster and greater response in ISR backscatter at the HF beam pointing along the magnetic field than at vertical [Isham et al., 1999b; Oyama et al., 2007]. Next, the excitation of the artificial aurora in the 
underdense ionosphere at $f_{0}$ above the $F_{2}$-peak plasma frequency foF2 [Pedersen et al., 2003; Mishin et al., 2005a; Kosch et al., 2007a]. Third, the overshoot behavior, i.e., the transient response of the ISR backscatter over a few seconds, unless the transmitter frequency $f_{0}$ is in the forbidden band near the gyroresonance $f_{0}=s f_{c e}$. Here $f_{c e}$ is the electron cyclotron frequency and $s \geq 1$ is an integer. The overshoot feature is due to anomalous absorption in the upper hybrid layer, which is inhibited near the gyroresonance [Mjølhus, 1993; Stubbe, 1996; Honary et al., 1999].

The high-power (>200 MW ERP) experiments also reveal the MZ and underdense ionosphere features. However, contrary to the low-power experiments, the ISR backscatter persists during the whole heating on period regardless of the relation of $f_{0}$ to $s f_{c e}$, particularly in the events of artificial ionization (section 4.6). This points to mitigation of anomalous absorption at high ERPs.

This chapter gives a concise survey of the conventional theoretical approach guiding the understanding of the observations in subsequent chapters. We do not dwell on theoretical details giving only the basic concepts on a semi-qualitative level, sufficient for understanding experimental results. Details and rigorous derivations can be found in the referenced original papers, reviews, and textbooks. Before describing principal nonlinear processes, the basics of the linear theory are outlined.

\subsection{Propagation of O-Mode Waves}

The free space field of an incident wave at the distance $R$ from a transmitter in the absence of absorption along the path from the ground is

$$
E_{f s}\left[\frac{V}{m}\right] \approx 5.5 \sqrt{P_{0}[\mathrm{MW}]} / \mathrm{R}[\mathrm{km}]
$$

O-mode HF beams pointed vertically and within the Spitze cone, $\left|\theta_{0}\right|<\theta_{s}$, reach the reflection point $h_{0}$. Here $\theta_{s}=\arcsin \left(\sqrt{\frac{f_{c e}}{f_{c e}+f_{0}}} \sin \chi\right)$ is the Spitze angle and $\chi \approx 14.5^{\circ}$ at HAARP and $12^{\circ}$ at Troms $\varnothing$ is the conjugate of the magnetic dip angle. In the northern hemisphere (downward $\mathbf{B}_{o}$ ), injection angles $\theta_{0}$ are positive to the south of vertical. Near $\theta_{0}=+\theta_{s}\left(-\theta_{s}\right)$, O-mode waves are converted to slow extraordinary waves, the $\mathrm{Z}$ mode moving upward (downward) [e.g., Mjølhus, 1990].

The wave amplitude swells near the reflection point $h_{0}$ due to the conservation of the Poynting flux, while the wave becomes nearly electrostatic and aligned with $\mathbf{B}_{o}$. Interference of incident and reflected waves forms a standing wave, the Airy pattern, $E(h)=A i\left(\frac{h-h_{0}}{l_{A}}\right)$, with the scale length $l_{A} \approx\left(c^{2} L_{n} \sin ^{2} \chi / \omega_{0}^{2}\right)^{1 / 3}$. In the first Airy maximum at $h_{A} \approx h_{0}-l_{A}$, the ratio of the amplitude $E_{A}$ to $E_{f s}$ (eq. 1), the swelling factor, is 


$$
\Gamma_{0}=\frac{E_{A}}{E_{f s}} \approx\left(\frac{2 \pi}{\sin \chi}\right)^{2 / 3}\left(\frac{f_{0} L_{n}}{c}\right)^{1 / 6}>>1
$$

For injections outside the Spitze cone, the O-mode is refracted near the altitude $h_{r}\left(\theta_{0}\right)$ which decreases with $\theta_{0}$. Albeit one should consider the caustic rather than the turning point [Mjølhus

et al., 2003], $A i\left(\frac{h-h_{0}}{l_{\theta}}\right)$ remains a good representation for the swelling pattern. Full-wave modeling shows that at $\theta_{0}>\theta_{s}$ the scale length $l_{\theta}$ increases with $\theta_{0}$ and at $\mathrm{MZ} l_{\chi} \equiv l_{m z}$ nearly doubles $l_{A}$. The swelling coefficient for a finite-width two-dimensional (2-D) HF beam in the beam center, $\Gamma_{2 D} \approx 0.8 \Gamma_{0}$, is about $2 \mathrm{~dB}$ greater than for 1D beams [Mishin et al., 2016]. The distance between $h_{0}$ and $h_{m z}$ increases nearly linearly with the scale height as $\Delta h_{m z}=h_{0}-h_{m z} \approx 0.03 L_{n}$. These features are important for the understanding of the Langmuir processes near the plasma resonance (sections 3.4 and 3.7).

\subsection{Electrostatic Plasma Waves}

In a weakly-magnetized $\left(\omega_{c e}<<\omega_{p e}\right)$ plasma, the dispersion relation of oblique HF plasma waves (henceforth "Lo" waves) away from the gyroharmonics, $s \omega_{c e}$, is

$$
\omega_{\mathbf{k}} \approx \omega_{p e}\left(1+\frac{3}{2} k^{2} r_{D}^{2}+\frac{\omega_{c e}^{2}}{2 \omega_{p e}^{2}} \sin ^{2} \theta\right)
$$

Here $\mathbf{k}$ is the wavenumber, $\theta=\arccos \left(\mathbf{k} \cdot \mathbf{B}_{0} / k B_{0}\right)$ is the propagation angle, and $r_{D}$ is the Debye radius. Equation (3) reduces to the Langmuir (L) branch, $\omega_{l} \approx \omega_{p e}\left(1+\frac{3}{2} k^{2} r_{D}^{2}\right)$ at $\theta=0$. For the upper hybrid branch at $\theta=\pi / 2$ and the harmonic number $s \geq 3$, one has

$$
\begin{gathered}
\omega_{u h} \approx \omega_{u h r}+\frac{3 \omega_{p e}^{2} \omega_{c e}^{2} x_{\perp}}{2 \omega_{u h r}\left(\omega_{u h r}^{2}-4 \omega_{c e}^{2}\right)} \text { at }\left|\delta_{u h r}\right| \gg \frac{\omega_{c e}}{\omega_{u h r}}\left(\frac{x_{\perp}^{s-1}}{2^{s-1} s !}\right)^{1 / 2} \\
\omega_{u h / e b}^{2} \approx s^{2} \omega_{c e}^{2} \pm s \omega_{p e} \omega_{c e}\left(\frac{x_{\perp}^{s-1}}{2^{s-1} s !}\right)^{1 / 2} \text { at } \delta_{u h r}=0
\end{gathered}
$$

Here $r_{c e}$ is the electron Larmour radius, $x_{\perp}=k^{2} r_{c e}^{2}<<1$, and $\delta_{u h r}=\omega_{u h r} / s \omega_{c e}-1$. In equation (5), $\omega_{e b}$ ("-") is the frequency of the electron Bernstein (EB) mode near double resonance $\omega=\omega_{u h r}=s \omega_{c e}$ or $\delta_{u h r}=0$. In general, EB waves have the group velocity $\partial \omega_{\mathbf{k}} / \partial k_{\perp}<0$ and the frequency close to the gyroresonance $s \omega_{c e}$ for both $k_{\perp} \rightarrow 0$ and $k_{\perp} \rightarrow \infty$. 
The dispersion near the second gyroharmonic should be treated more carefully to avoid the singularity [Grach, 1979]. Figure 3.1 shows the dispersion curves near $2 f_{c e}$ and $3 f_{c e}$ for $\omega_{u h r}=s \omega_{c e}$ (solid lines), $\omega_{u h r}>s \omega_{c e}$ (dashed), and $\omega_{u h r}<s \omega_{c e}$ (dotted) calculated at $k_{\|}=0$ from the general dispersion relation. At $\delta_{u h r} \neq 0$, the curves approach $f_{u h r}$ when $k_{\perp} \rightarrow 0$. Note that only the EBmode waves with $\partial \omega_{\mathbf{k}} / \partial k_{\perp}<0$ exist below $2 \omega_{c e}$. This is a key feature for the dynamics of Langmuir turbulence and artificial ionization layers at heating near the second gyroharmonic (section 4.6).

For electrostatic waves, the wave energy density $W_{\mathbf{k}}$ is the sum of the electrostatic energy $\left|E_{\mathbf{k}}\right|^{2} / 8 \pi$ and the average kinetic energy of the oscillatory (quiver) motion $\bar{\varepsilon}_{k i n}=\sum_{j} n_{0} m_{j} \bar{v}_{j}^{2} / 2$. Here $\bar{x}$ denotes the averaging of a function $x(t)$ over the wave period. For Langmuir oscillations in cold plasma, one gets $\bar{\varepsilon}_{k i n}=\left|E_{\mathbf{k}}\right|^{2} / 8 \pi$ so $W_{l}=\left|E_{\mathbf{k}}\right|^{2} / 4 \pi$ is twice the electrostatic energy. Simply put, charge separation due to electron motion sets up the electric field to restore charge neutrality and thus the average potential

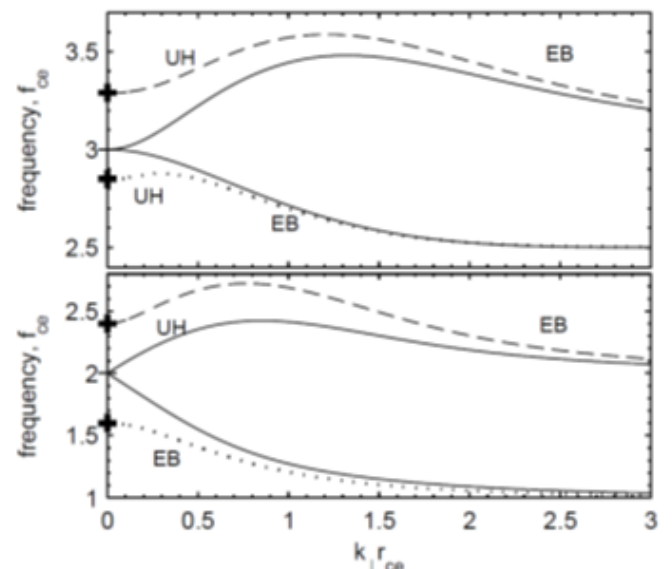

Figure 3.1. Dispersion curves near $3 f_{c e}$ (top) and $2 f_{c e}$ (bottom) for $\delta_{u h r}=0$ (solid lines), $>0$ (dashed), and $<0$ (dotted). Signs "+" designate $f_{\text {uhr }}$. After [Mishin et al., 2005b]. and kinetic energies are equal. Similarly, the energy density of UH oscillations is $W_{u h}=\left(\omega_{u h} / \omega_{p e}\right)^{2}\left|E_{\mathbf{k}}\right|^{2} / 4 \pi$.

The low-frequency (LF), $\Omega<<\omega_{c e}$, modes of interest are ion sound (S) and lower hybrid (LH) waves

$$
\begin{aligned}
& \Omega_{s}=q c_{s} /\left(1+q^{2} r_{D}^{2}\right)^{1 / 2} \text { at } T_{e}>3 T_{i} \\
& \Omega_{l h}=\Omega_{l h r} \sqrt{1+q^{2} r_{c^{*}}^{2}+\mu^{-1} q_{z}^{2} / q^{2}}
\end{aligned}
$$

Here $T_{i} / T_{e}$ is the ion/electron temperature, $c_{s}=\sqrt{\left(1+3 \frac{T_{i}}{T_{e}}\right) T_{e} / m_{i}}$ is the ion sound speed, $\Omega_{l h r} \approx \mu^{1 / 2} \omega_{c e}$ at $\omega_{c e}<<\omega_{p e}, \quad r_{c *}^{2}=\left(\frac{3}{4}+\frac{3 T_{i}}{T_{e}}\right) r_{c e}^{2}$, and $\mu=m_{e} / m_{i} \approx 3 \cdot 10^{-5}$ is the electron-to-ion mass ratio in the $\mathrm{F}$ region.

At $k^{2} r_{D}^{2}<<1$, one has $\Omega_{s} \approx k c_{s}$ and $\delta N_{e} \approx \delta N_{i}$, as electrons shield out slowly-changing ion charges. The wave energy density is $W_{s}=\mu \frac{\omega_{p e}^{2}}{\Omega_{k}^{2}}\left|E_{\mathbf{k}}\right|^{2} / 8 \pi$. If $T_{e} \rightarrow 0$, charge fluctuations are carried by chaotically-moving thermal ions (ion quasimodes) and heavily damped, i.e., $\operatorname{Im} \Omega \sim \operatorname{Re} \Omega$. Actually, the latter occurs already at $T_{e}<3 T_{i}$. As opposed to ion sound waves, $\mathrm{LH}$ 
waves with the energy density $W_{l h} \approx \frac{\omega_{p e}^{2}}{\omega_{c e}^{2}}\left|E_{\mathbf{k}}\right|^{2} / 8 \pi$ exist even at $T_{i} \geq T_{e}$.

\subsubsection{Wave-Particle Analogy}

It is instructive for better understanding of nonlinear wave coupling to recall the wave-particle quantum-mechanical analogy. Since $\omega_{\mathbf{k}}(\mathbf{r}, \mathbf{k})$ is constant in stationary media, we have $d \omega_{\mathbf{k}}=\left.d \mathbf{r} \frac{\partial \omega_{\mathbf{k}}}{\partial \mathbf{r}}\right|_{\mathbf{k}}+\left.d \mathbf{k} \frac{\partial \omega_{\mathbf{k}}}{\partial \mathbf{k}}\right|_{\mathbf{r}}=0$ or

$$
\frac{d}{d t} \mathbf{r}=\mathbf{v}_{g}=\frac{\partial}{\partial \mathbf{k}} \omega_{\mathbf{k}} ; \frac{d}{d t} \mathbf{k}=-\frac{\partial}{\partial \mathbf{r}} \omega_{\mathbf{k}}
$$

That is, the wave vector changes in such a way that the frequency is preserved. Equations (8) are the Hamilton equations for a unit-mass particle (plasmon) with the "energy" $\omega_{\mathbf{k}}$ and "momentum" $\mathbf{k}$, moving with the (group) velocity $\mathbf{v}_{g}$. Let us consider Langmuir plasmons moving in a one-dimensional density depletion (cavity) $n(x)=n_{0}(1+\delta N(x))$ with $\delta N(x) \leq 0$ centered at $x=x_{*}$, with the width $l_{n} \gg k_{*}^{-1}\left(k_{*}=k\left(x_{*}\right)\right)$. Expanding $\omega_{p e}(n)$ for $|\delta N| \ll 1$ gives

$$
\frac{\omega_{l}(n(x))}{\omega_{p 0}}-1 \approx \frac{1}{2} \delta N(x)+\frac{3}{2} k^{2}(x) r_{D}^{2}
$$

The plasmons' Hamiltonian is the sum of the kinetic energy, $\varepsilon_{k}=\mathbf{k}^{2} / 2$, and potential, $U(x)=2 \delta N(x) / 3 r_{D}^{2}$. "Acceleration" $\left(\frac{d}{d t} \mathbf{k}=\mathbf{v}_{g} \frac{\partial}{\partial \mathbf{r}} \mathbf{k}>0\right)$ and "deceleration" $\left(\frac{d}{d t} \mathbf{k}<0\right)$ for plasmons moving toward and from the center, can be seen from eq. (8). Evidently, the cavity is a potential hole for plasmons that are trapped if the Hamiltonian is negative, i.e., $-U\left(x_{c}\right)>\varepsilon_{k}\left(x_{*}\right)$ or

$$
\left|\delta N\left(x_{*}\right)\right|>3 k_{*}^{2} r_{D}^{2},
$$

and move freely otherwise. This condition remains valid for low frequency, such as ion sound, density oscillations and is in the basis of parametric coupling of HF and LF plasma waves outlined below.

\subsection{Ponderomotive Parametric Instability (PPI)}

Maxwell's equations are linear, so waves in plasma with a given dielectric permittivity are independent. The latter is not valid, however, if the plasma dispersive properties depend on the wave fields. For small wave amplitudes, one can use the perturbation theory to obtain the first order linear eigenmodes, i.e., wave packets centered at the eigenfrequencies $\omega_{\mathbf{k}}$ [e.g., Galeev and Sagdeev, 1979]. In the next order, the particle equations of continuity and motion and hence the wave equations will include products $\mathbf{E}_{\omega_{\mathrm{k}} \mathbf{k}} \mathbf{E}_{\omega_{\mathbf{q}} \mathbf{q}}$. Thus, as for the parametric resonance of two tied oscillators, HF plasma modes, $\hat{k}_{1}=\left(\mathbf{k}_{1}, \omega_{\mathbf{k}_{1}}\right)$, can be parametrically coupled to an imposed (pump) 
HF wave, $\hat{k}_{0}=\left(\mathbf{k}_{0}, \omega_{0} \approx \omega_{\mathbf{k}_{1}}\right)$. Yet, matching only the frequencies is not sufficient as $\mathbf{k}_{1}$ and $\mathbf{k}_{0}$ can differ greatly. That is why LF modes, $\hat{q}=\left(\mathbf{q}, \Omega_{\mathbf{q}}\right)$, are needed.

The wave parametric resonance (matching) condition

$$
\mathbf{k}_{1}=\mathbf{k}_{ \pm}=\mathbf{k}_{0} \pm \mathbf{q} \text { and } \omega_{\mathbf{k}_{ \pm}}=\omega_{0} \pm \Omega_{\mathbf{q}}
$$

is easily understood using the wave-particle analogy. Namely, decay of the "mother"-quantum $\left(\varepsilon_{0}, \mathbf{p}_{0}\right)$ into two "daughter"-quanta proceeds with the conservation of the total energy, $\varepsilon_{0}=\varepsilon_{\mathbf{p}_{1}}+\varepsilon_{\mathbf{p}_{2}}$, and momentum, $\mathbf{p}_{0}=\mathbf{p}_{1}+\mathbf{p}_{2}$. Therefore, a three-wave process, $\hat{k}_{0} \rightarrow \hat{k}_{1}+\hat{q}$, is called parametric decay instability (PDI) which excites the red-shifted (or Stokes) sideband, $\hat{k}_{-}=\hat{k}_{0}-\hat{q}$. A strict analogy for the blue-shifted (anti-Stokes) sideband, $\hat{k}_{+}=\hat{k}_{0}+\hat{q}$, is coalescence of the $\hat{k}_{0}$ and $\hat{q}$ waves.

The key element of parametric coupling is the ponderomotive (striction) force arising from the beating of the HF pump and plasma waves. Therefore, for brevity, we call the overall process ponderomotive parametric instability (PPI). In isotropic plasma, with $\omega_{0} \sim \omega_{1} \sim \omega_{p e}$ the total HF field, $\mathbf{E}(t, \mathbf{r})=\mathbf{A}(t, \mathbf{r}) \cos \left(\mathbf{k r}-\omega_{p e} t\right)$ with $\frac{\partial}{\partial t} \ln |\mathbf{A}|<<\omega_{p e}$, exerts in the first approximation the electron quiver velocity $\mathbf{u} \approx-\frac{e}{m_{e} \omega_{0}} \mathbf{A} \sin (\mathbf{k r}-\omega t)$. Averaging the second order term $m_{e}(\mathbf{u} \nabla) \mathbf{u}$ in the electron fluid equation over the fast time $\sim \omega_{p e}^{-1}$ replaces $T_{e}$ by $T_{e}+p_{h f} / n_{e}$, with the HF field pressure $p_{h f}$ giving the sought-for force

$$
\mathbf{F}_{p}=-\frac{1}{n_{e}} \nabla p_{h f}=-\nabla \frac{|\mathbf{A}|^{2}}{16 \pi n_{e}}
$$

The quantitative measure of $p_{h f}$ is the parameter of nonlinearity

$$
w=\frac{W}{n_{e} T_{e}}=\frac{|\mathbf{A}|^{2}}{8 \pi n_{e} T_{e}}
$$

Another key parameter is the pump frequency mismatch

$$
\Delta_{0}=\frac{\omega_{0}}{\omega_{p e}}-1
$$

as two HF oscillations with close frequencies $\omega_{0} \approx \omega_{1}$ exert the ponderomotive force at the beat frequency $\Omega=\omega_{0}-\omega_{1}$.

The PPI mechanism is as follows. The pump wave exerts the quiver velocity $\mathbf{u}$ (HF pressure) on electrons that couples with the LF density perturbation $\delta N$ as the excess of the electron pressure sets up the ambipolar electric field to maintain charge neutrality. A nonlinear current, $-e \delta n_{s} \cdot \mathbf{u}$, drives the HF plasma wave, which connects with the pump to drive the LF 
mode via the ponderomotive force $\mathbf{F}_{p}$, thereby closing the positive-feedback loop. Depending on the input parameters, PPI can develop as a three-wave process, i.e. PDI, or a four-wave process termed modulational instability (MI), also known as the oscillating two-stream instability (OTSI) [e.g., Galeev et al., 1977; Fejer, 1979; Shapiro and Shevchenko, 1984; DuBois et al., 2001]. The term "MI" underscores that an initially uniform wave envelope becomes modulated.

\subsubsection{PPI in Isotropic Plasma (PPI $)_{L}$}

For illustration, we consider isotropic plasma subjected to a large-scale, $k_{0} r_{D}<<1$, monochromatic Langmuir (pump) wave $\mathbf{E}_{0}=\mathbf{A}_{0} e^{i\left(k_{0} z-\omega_{0} t\right)}$. The ponderomotive force (eq. 11) stirs slow density fluctuations, $\delta N_{s}=n_{i} / n_{0}-1$, that affect Langmuir plasmons via (eq. 8). The PPI $L$ description is based on the Zakharov equations that isolate slow processes by filtering out the fast (HF) variations [e.g., Zakharov, 1984]. For that, the Langmuir field, $\mathbf{E}_{l}=-\nabla \phi$, is presented as $\phi=\operatorname{Re}\left(\Psi(r, t) e^{-i \omega_{p e} t}\right)$ with envelopes $\Psi=\Psi_{0}+\Psi_{1}$ and $\mathbf{A}_{l}=-\nabla \Psi$, with $\Psi_{j}=\Psi_{k_{j}} e^{i\left(\mathbf{k}_{j} \mathbf{r}-\delta_{\mathbf{k}_{j}} t\right)}$ and $\delta_{\mathbf{k}_{1,0}}=\omega_{l}\left(\mathbf{k}_{1,0}\right)-\omega_{p e} \sim \Omega<<\omega_{p e}$. Given the above wave-particle analogy, the wave equations can be derived from the dispersion relations replacing frequencies by $-i \partial / \partial t$ and wave vectors k by $i \nabla$. Then, substituting $n_{e}=n_{0}\left(1+\delta N_{s}\right)$, with $\delta N_{s}=\delta N_{q} e^{i(\mathbf{q r}-\Omega t)}$, into the HF dispersion (eq. 3) with $B_{0}=0$ and averaging over $\omega_{p e}^{-1}$ yields the first Zakharov equation [e.g., Robinson, 1997]

$$
\left(i \frac{\partial}{\partial t}-i \frac{v_{l}}{2}+\frac{3}{2} \omega_{p e} r_{D}^{2} \nabla^{2}\right) \nabla^{2} \Psi=\frac{\omega_{p e}}{2} \nabla\left(\delta N_{s} \mathbf{A}\right)
$$

where $\mathbf{A}=-\nabla \Psi+\mathbf{A}_{0}$. Adding the ponderomotive force (eq. 11) into (eq. 6) replaces $T_{e}$ by $T_{e}+p_{h f} / n_{0}$ to give the second Zakharov equation for the slow density variation $\delta N_{s}$

$$
\left(\frac{\partial^{2}}{\partial t^{2}}+v_{s} \frac{\partial}{\partial t}-c_{s}^{2} \nabla^{2}\right) \delta N_{s}=\frac{1}{16 \pi n_{0} m_{i}} \nabla^{2}|\mathbf{A}|^{2}
$$

Here $v_{l, s}$ is the collisional damping rate of Langmuir (ion sound) waves, while Landau damping is neglected.

Linearizing (eq. 14-15) in $\delta N_{s}$ and $\left|\Psi_{1}\right|$ yields the dispersion equation for the LF density response coupled with the Langmuir sidebands $\left(\omega_{l}\left(\mathbf{k}_{ \pm}\right), \mathbf{k}_{ \pm}=\mathbf{k}_{0} \pm \mathbf{q}\right)$ via the pump

$$
\Omega\left(\Omega+2 i v_{s}\right)-\Omega_{\mathbf{q}}^{2}=\frac{\omega_{p e}}{4} \Omega_{\mathbf{q}}^{2} \sum_{ \pm} \frac{w_{0} \cos ^{2} \alpha_{ \pm}}{\delta_{ \pm} \pm \Omega \pm i v_{ \pm}}
$$

Here $w_{0}=\frac{w_{0}}{n_{0} T_{e}}, \delta_{ \pm}=\omega_{0}-\omega_{l}\left(\mathbf{k}_{ \pm}\right), v_{ \pm}=v_{l}\left(k_{ \pm}\right)$, and $\alpha_{ \pm}$is the angle between $\mathbf{k}_{ \pm}$and $\mathbf{E}_{0}$. This equation is valid also for electromagnetic pump waves near the plasma resonance. 


\subsubsection{Parametric Decay Instability $\left(\mathrm{PDI}_{L}\right)$}

Let us start with small $w_{0}$ when only the red-shifted sideband $\omega_{l}\left(\mathbf{k}_{-}\right)$is in resonance, $\Delta_{k}=\delta_{-}-\Omega_{\mathbf{q}} \rightarrow 0$, with the pump, while $\Sigma_{+}$is negligible. At the resonance, substituting $\Omega=\Omega_{\mathbf{q}}+\delta \Omega+i \gamma_{n l}$ and $v_{l, s}=0$ in (eq. 16) yields the $\mathrm{PDI}_{\mathrm{L}}$ growth rate

$$
\gamma_{n l} \equiv \gamma_{d} \approx \omega_{p e} \sqrt{\Omega_{\mathbf{q}} w_{0} / 8 \omega_{0}}
$$

For brevity, we use $w_{0}$ instead of $w_{0} \cos ^{2} \alpha_{-}$.

The resonance condition $\Delta_{k}=0$ can be satisfied only for the short-scale pump, $k_{0}>k_{*}=\sqrt{\mu} / 3 r_{D}$. If $k_{0} \gg k_{*}$, then $\mathbf{q} \approx 2 \mathbf{k}_{0}$ and hence $\mathbf{k}_{-} \approx-\mathbf{k}_{0}$, i.e., the "daughter" plasmon moves in the opposite direction. Accounting for damping $v_{l, s}$ gives the $\mathrm{PDI}_{\mathrm{L}}$ growth rate and threshold

$$
\gamma_{d}^{2}>v_{l} v_{s} \text { at } w_{0}>w_{d}=8 \frac{v_{l} v_{s}}{\omega_{p e} \Omega_{2 k_{0}}}
$$

For $\Delta_{k} \neq 0$ and $\gamma_{d}^{2}>>v_{l} v_{s}$, the growth rate becomes $\gamma_{\Delta k} \approx \sqrt{\gamma_{d}^{2}-\left(\Delta_{k} / 2\right)^{2}}$. Namely, $\mathrm{PDI}_{\mathrm{L}}$ develops inside the resonance zone $\left|\Delta_{k}\right| \leq 2 \gamma_{d}$. This condition defines the spectral width, $\delta k_{*}=k_{0}-k_{-} \approx 2 \mu^{1 / 2} / 3 r_{D}=2 k_{*}$, of the unstable wave spectrum.

At $T_{i} \sim T_{e}$, the ion Landau damping gives $v_{s} \sim \Omega_{q}$. For $v_{s}>\gamma_{d}>>v_{l}, \mathrm{PDI}_{\mathrm{L}}$ develops with a small growth rate

$$
\gamma_{\text {ind }} \approx \gamma_{d}^{2} / v_{s} \approx \omega_{p e} w_{0} / 8 \text { at } w_{0}>>w_{\text {ind }} \approx 4 v_{l} / \omega_{\text {pe }}
$$

with the spectral width $\delta k_{i n d} \sim 2 \sqrt{\mu T_{i} / T_{e}} / 3 r_{D}$. As $\Omega_{q} / q \approx\left(\omega_{0}-\omega_{\mathbf{k}}\right) /\left|\mathbf{k}_{0}-\mathbf{k}\right|$, this process is actually damping of beat waves called induced scattering or nonlinear Landau damping.

The blue-shifted sideband is out of the resonance or $\delta_{+}>|\Omega| \sim \gamma_{d}$, if two conditions are satisfied: $w_{0}<24 \sqrt{3} \mu^{-1}\left(k_{0} r_{D}\right)^{4}$ and $k_{0}>k_{*}$. If either of these conditions is violated, both sidebands are in resonance with the pump and thus a four-wave process (MI) takes place. A quantum-mechanical analogy for MI is an exchange of a phonon (an ion quasimode) between two plasmons.

\subsubsection{Modulational Instability (MI)}

For $k_{0}<<k_{*}=\mu^{1 / 2} / 3 r_{D}$, the dipole approximation (an uniform oscillating field), $k_{0}=0$, for the pump field can be used. That gives $\mathbf{k}_{ \pm}= \pm \mathbf{q}$, so both sidebands develop alike. As a result, equation (16) reduces to $\left(v_{l, s}=0\right)$ 


$$
\left(\frac{\Omega^{2}}{k^{2} c_{s}^{2}}-1\right)\left(\Omega^{2}-\delta_{k}^{2}\right)=\omega_{p e} \delta_{k} \frac{w_{0}}{2} \cos ^{2} \alpha
$$

where $\delta_{k}=\omega_{\mathbf{k}_{ \pm}}-\omega_{0} \approx \frac{3}{2} \omega_{p e} k^{2} r_{D}^{2}$. This equation is invariant for plasmons propagating either along or opposite to $\mathbf{E}_{0}$. Unstable solutions in both the subsonic $\left(|\Omega|<k c_{s}\right)$ and supersonic $\left(|\Omega|>k c_{s}\right)$ regimes exist above the MI threshold

$$
w_{0} \cos ^{2} \alpha>w_{t h}=3 k^{2} r_{D}^{2}
$$

The MI instability results from accumulation of Langmuir plasmons in cavities, $\delta N_{s}<0$. As $\delta p_{h f} \approx-W_{0} \delta N_{s} /\left(3 k^{2} r_{D}^{2}\right)$ inside a cavity exceeds the thermal pressure imbalance, $\delta p_{e}=n_{0} T_{e} \delta N_{s}$, the plasma is pushed out of the cavity which further deepens and traps yet more plasmons in a positive-feedback loop. In the subsonic regime $w_{0}<3 \mu \sim 10^{-4}$ and $k r_{D}<\sqrt{\mu}$, one gets from (eq. 15) $\delta N_{s} \approx-w_{0} / 2$ and the maximum growth rate $\gamma_{m s}=\omega_{p e} w_{0} / 4$ at $k^{2} r_{D}^{2}=w_{0} / 6$. Substituting $\delta N_{s}$ in (eq. 14) gives the nonlinear Schrödinger equation, commonly used in nonlinear optics, in particular describing self-focusing of laser beams.

In the supersonic regime, $w_{0}>3 \mu$, the growth rate maximizes at $k_{m} r_{D} \approx \mu^{1 / 2}\left(w_{0} / 3 \mu\right)^{1 / 3}$ and then reaches a plateau at $k_{m}<k \leq k_{t h} \approx r_{D}^{-1} \sqrt{w_{0} / 3}$ with

$$
\gamma_{m i} \approx \omega_{p e} \sqrt{\mu w_{0} / 3}
$$

which dominates the phase volume of the excited Langmuir plasmons. This regime is particularly important at high HF powers such as used for production of artificial ionization layers (section 4.6).

\subsection{PPI in the Plasma Resonance Layer}

At ERPs $P_{0}=0.4 p_{0} \geq 0.4 \mathrm{GW}$, the free space field (eq. 1) at $h_{0}=220 \mathrm{~km}$ is $E_{f s} \approx 0.5 p_{0}^{1 / 2}$ $\mathrm{V} / \mathrm{m}$. For $f_{0}=f_{p e}=3 \rightarrow 6 \mathrm{MHz}$ or the critical ("resonance") density $n_{c} \approx(1 \rightarrow 5) \cdot 10^{5} \mathrm{~cm}^{-3}$ and the scale height $L_{n}=40 \mathrm{~km}$, one gets at HAARP $E_{A}=\Gamma_{0} E_{f s} \approx 15 p_{0}^{1 / 2} \mathrm{~V} / \mathrm{m}$. For $T_{e}=0.2 \mathrm{eV}$, the parameter of nonlinearity (eq. 12) becomes $w_{A}=E_{A}^{2} / 8 \pi n_{0} T_{e} \approx(0.25 \rightarrow 0.06) p_{0}$; at EISCAT $w_{A}$ is slightly greater. Using the swelling coefficient $\Gamma_{2 D} \approx 0.8 \Gamma_{0}$ at $\mathrm{MZ}$ yields $w_{m z} \approx(0.15 \rightarrow 0.04) p_{0}$ - The pump frequency mismatch (eq. 13) at vertical and $\mathrm{MZ}$ is $\Delta_{A} \approx l_{A} / 2 L_{n} \sim 10^{-3}$ and $\Delta_{m z} \approx \Delta h_{m z} / 2 L_{n} \approx 0.015$, respectively. As $\Delta_{A, m z}<<w_{A, m z}$, the pump frequency mismatch in the first few Airy maxima can be neglected.

Near the reflection point, the pump electric field is nearly along $\mathbf{B}_{0}$, and as $\omega_{0} / c<<k_{*}=\mu^{1 / 2} / 3 r_{D}$ the dipole approximation can be used. The ponderomotive force is 
virtually the same as $\mathbf{F}_{p}$ (eq. 11) with $p_{h f}$ of the total, pump and electrostatic, $\mathrm{HF}$ wave field. As ions are unmagnetized, equation for $\delta N_{i}$ (eq. 15) remains. At $\omega_{c e}^{2} / \omega_{p e}^{2}<<1$, the magnetic correction for oblique Langmuir waves (eq. 3) adds the term $\frac{-\omega_{c e}^{2}}{2 \omega_{p e}} \nabla_{\perp}^{2} \Psi$ in the left hand side of (eq. 14). Accordingly, in the dispersion equation (eq. 20) only the terms $\delta_{ \pm}$are modified to include $\left(\omega_{c e}^{2} / 2 \omega_{p e}^{2}\right)\left(\mathbf{k}_{ \pm \perp}^{2} / \mathbf{k}_{ \pm}^{2}-\mathbf{q}_{\perp}^{2} / \mathbf{q}^{2}\right)$ [e.g., Shapiro and Shevchenko, 1984].

The MI development at MZ and vertical is similar at $p_{0} \geq 1$. At such powers, collisional and convective damping can be neglected as $w_{A, m z}>>\max \left(4 v_{e} / \omega_{p e}, 2 / k L_{h}\right) \sim 10^{-4} \sim 3 \mu$. Here $k$ is the excited wavenumber $\left(>10^{-2} / r_{D}\right)$ and $v_{e} \sim(3-5) \times 10^{2} \mathrm{~s}^{-1}$ is the electron collision frequency. At $w_{0} \ll \omega_{c e}^{2} / \omega_{p e}^{2}$, the instability develops in a narrow cone $|\theta| \leq \frac{\omega_{p e}}{\omega_{c e}} w_{0}^{1 / 2}<<1$ around $\mathbf{B}_{0}$ with nearly the same growth rate (eq. 22) $\gamma_{m i} \sim 10^{-3} \omega_{p e} p_{0}^{1 / 2} \sim 10^{4 \rightarrow 5} \mathrm{~s}^{-1}$, and as in isotropic plasma otherwise.

So far, the excited "daughter" waves are implied non-interacting and uncorrelated. After they grow over the thermal noise level, their parametric interactions become significant. Further description depends on whether the waves are coherent (correlated) or random. Random waves can be described statistically, replacing averaging over time and space by that over the statistical ensemble. In general, the choice depends on the bandwidth of the excited waves $\Delta \omega$ and the rate of nonlinear coupling (phase mixing) $\gamma_{n l}$. As a rule of thumb, for weak nonlinearities and broad spectra, $\Delta \omega>>\gamma_{n l}$, the waves can be treated as weakly correlated. Therefore, this regime is called weak-turbulence (WT). In the opposite limit, $\Delta \omega \leq \gamma_{n l}$, the waves are strongly correlated and hence it is the strong turbulence regime.

We present next a heuristic description of strong Langmuir turbulence (SLT) near the plasma resonance, $h_{0}$, typically at $200-250 \mathrm{~km}$. The SLT regime has distinct features, such as the "caviton continuum" and the central peak in the incoherent radar PL and IL spectra, respectively, and the Narrow Continuum (NC) in the SEE spectrum (section 4.1) [Stubbe, 1996; Isham et al., 1999a; Mjølhus et al., 2003; Djuth and DuBois, 2015; Grach et al., 2016]. Most important for the artificial plasma production (section 4.6) is the SLT acceleration of suprathermal (tail) electrons.

\subsubsection{Strong Langmuir Turbulence (SLT)}

As soon as $\left|\delta N_{s}\right|>3 k^{2} r_{D}^{2}$, plasmons are trapped inside a cavity, so a localized wave packet is formed. Cavities with trapped strongly-correlated Langmuir oscillations, termed Langmuir cavitons or simply cavitons, constitute strong Langmuir turbulence. The dynamics of cavitons depends on the dimensionality of the problem as follows [e.g., Sagdeev, 1979].

The conservation of the plasmons' number $\sim \int d^{3} \boldsymbol{r}|\mathbf{E}|^{2} / \omega_{p e}$ in an isotropic cavity $\left|\delta N_{s}\right|<<1$ 
of the size $l$ yields $\left|\mathbf{E}^{2}(t)\right| \propto l^{-D}(t)$. The wavelengths of the trapped plasmons are also of the order of $l$ so that their wavenumbers $k \sim 1 / l$. As $l^{-2} \sim k^{2} \propto\left|\delta N_{s}\right|$, a deepening cavity narrows (collapses) with time. Since $\left|\delta p_{e}\right| \propto T_{e}\left|\delta N_{s}\right| \propto l^{-2}$ and $\left|\mathbf{E}^{2}\right| \propto l^{-D}$, the thermal pressure will ultimately balance the HF pressure for $D=1$, thus forming one-dimensional cavitons. In two (three) dimensions, the speed of collapse persists (accelerates) with time.

The phase velocity of plasmons in collapsing cavitons $\sim \omega_{p e} / k_{\|}(t)$ decreases with time, and at some absorption size, $l_{\|} \leq l_{a} \sim k_{a}^{-1} \sim(3-5) r_{D}$, plasmons are absorbed by plasma electrons due to Landau and transit-time damping. As a result, a small group of suprathermal electrons gains energy, while the HF pressure in the caviton drops and collapse is arrested due to the wave energy "burnout". Ultimately, the dynamic equilibrium is reached between the pumping energy into cavitons in the long-scale source region, $k_{L} \leq r_{D}^{-1} \sqrt{w_{L} / 3}$, and short-scale transfer by collapsing cavitons $\left(W_{\text {cav }}\right)$ through the inertial interval into the

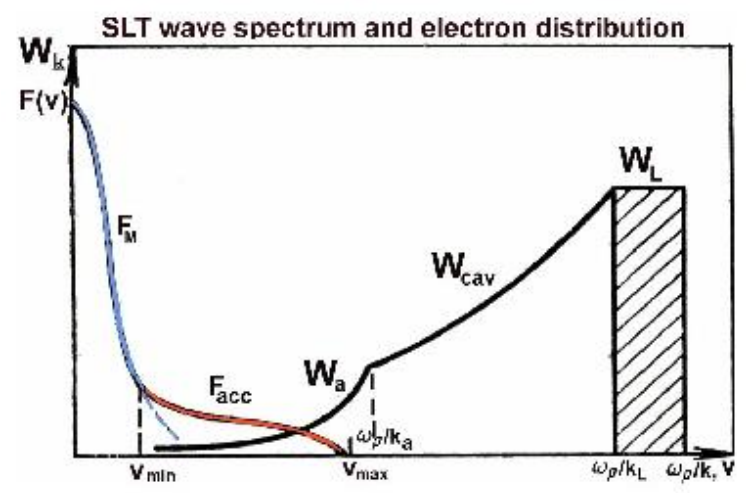

Figure 3.2. Schematic representation of the wave spectrum and electron distribution in the developed strong Langmuir turbulence [Shapiro and Shevchenko, 1984]. absorption interval $k \geq k_{a}$ [Galeev et al., 1977]. The energy density in the source region, $W_{L},($ $W_{L}>W_{c a v}>W_{a}=\int_{k \geq k_{a}} W_{k} d k$ ) comprises the MI-excited (non-trapped) long-scale waves. The total energy of turbulent electric fields in saturation is reduced relative to the initial stage (so-called SLT overshoot). Figure 3.2 shows a schematic representation of the SLT scenario [Galeev et al., 1977; Shapiro and Shevchenko, 1984].

Since slow $\left(\Omega \sim \gamma_{m i}<<\omega_{c e}\right.$ ) electron motion is magnetized, the cavitons at $\left|\delta N_{s}\right|<<\omega_{c e}^{2} / \omega_{p e}^{2}$ are pancake-like. Their dimensions

$$
l_{\|} \sim k_{\|}^{-1} \sim r_{D}\left|\delta N_{s}\right|^{-1 / 2} \sim l_{\perp} \frac{\omega_{p e}}{\omega_{c e}}\left|\delta N_{s}\right|^{1 / 2}
$$

are obtained by balancing the terms $\omega_{p e} r_{D}^{2} \nabla^{4} \Psi$ by $\frac{\omega_{c e}^{2}}{\omega_{p e}} \nabla_{\perp}^{2} \Psi$ and using the trapping condition $\left|\delta N_{s}\right| \sim k_{\|}^{2} r_{D}^{2} \sim r_{D}^{2} l_{\|}^{-2} \quad$ [Shapiro and Shevchenko, 1984]. The self-similar solution is $l_{\|}^{2} \propto l_{\perp} \propto\left|\delta N_{s}\right|^{-1}$ with the initial dimensions defined by equation (23) with $\left|\delta N_{s}\right| \sim w_{L}$. If the density variation $\left|\delta N_{s}\right|$ in collapsing cavitons reaches $\omega_{c e}^{2} / \omega_{p e}^{2}$ at $l_{\|}^{-1}<<k_{a}$, the cavitons become symmetric $\left(l_{\perp} \sim l_{\|}\right)$and continue to collapse with the wave energy density 
$W_{s y m} \sim W_{L} \frac{\omega_{p e}}{\omega_{c e}} \sqrt{w_{L}}<<W_{L}$ until the energy burnout.

The above scenario fails for $w_{L}>3 k_{a}^{2} r_{D}^{2} \sim 0.1$ or $w_{0}>w_{s s t} \sim \sqrt{3 \mu} \sim 10^{-2}$. In this "superstrong" (SST) regime, MI develops in the whole region from the pump to absorption so collapse plays a minor role in the energy balance [Shapiro and Shevchenko, 1984]. Another limitation concerns the role of small-scale, $q>>k_{L}$, random density irregularities, $\delta N(\mathbf{r})=\sum_{q} \operatorname{Re}\left(\delta N_{q} \exp (i \mathbf{q r})\right)$. In particular, small-scale $\left(q \sim k_{a}\right)$ ion sound waves radiated by burned-out cavitons can accumulate at $T_{e}>3 T_{i}$ [Galeev et al., 1977]. As a result of conversion, $L_{k_{L}}+\delta N \rightarrow L_{q}^{\prime}$, with the rate

$$
\gamma_{c} \approx \sum_{\mathbf{q}} v_{l}(\mathbf{q}) \frac{\left\langle|\delta N|^{2}\right\rangle_{\mathbf{q}}}{36 q^{4} r_{D}^{4}}<<v_{l}(\mathbf{q})
$$

the large-scale plasmons are converted into the small-scale plasmons circumventing the inertial interval. Here $v_{l}(\mathbf{k})$ is the total (collisional + Landau) damping rate and $\left\langle|\delta N|^{2}\right\rangle_{\mathbf{q}}$ is the phaseaveraged spectral energy. The same procedure describes resonant scatter of O-mode waves on either FAI near $h_{u h}$ [Mishin et al., 2001] or SLT cavitons near $h_{r}$ [Eliasson, 2013] into oblique Zand O-mode waves.

\subsubsection{Coexistence of WT and SLT Regimes}

It was so far implied that $\Delta_{0}=0$ and $\delta_{k} \approx \omega_{p e} \frac{3}{2} k^{2} r_{D}^{2}$. However, for an electromagnetic pump near the cutoff point with a finite value of $\Delta_{0}$, either positive or negative $\delta_{k} \approx \omega_{p e}\left(\frac{3}{2} k^{2} r_{D}^{2}-\Delta_{0}\right)$ should be assumed. This is significant for the instability development at $\Delta_{0}>w_{0}>>\mu$ [Shapiro and Shevchenko, 1984]. For $\Delta_{0}>w_{0}>>\sqrt{\mu \Delta_{0}}$, two regimes exist at $\delta_{k}>0$ and $\delta_{k}<0$. In the latter case, the modified decay instability develops for $\left|\delta_{k}\right|<\gamma_{1} / 2^{1 / 3}$, where the growth rate $\gamma_{1} \approx \gamma_{m i}\left(W_{0}, k\right)$ (eq. 22). For $\delta_{k}>0 \quad\left(\Omega^{2}<0\right)$, the growth rate reaches maximum $\gamma_{2} \approx \gamma_{m i}\left(W_{0}, k_{p}\right) / \sqrt{3}$ at $\delta_{k_{p}}=2^{1 / 3} \gamma_{1}$. Note that $\gamma_{1,2}<<\Delta_{0}$ so $k_{p} \sim r_{D}^{-1} \sqrt{2 \Delta_{0} / 3}$. In the region of modulational instability at $0<2 \delta_{k} / \omega_{p e}<w_{0}$, which occupies the greatest phase volume, the growth rate is $\gamma_{m k} \approx \omega_{p e} \mu^{1 / 2} k r_{D}$. Here, the development of turbulence is similar to that at $\Delta_{0}=0$; the small-scale wave energy is smaller though.

For large mismatches $\Delta_{0}>w_{0}^{2} / \mu$ and $T_{e} \sim T_{i}$, induced scattering on ions at $w_{0}>w_{\text {ind }}$ generates (primary) plasmons at $k \sim k_{m}>k_{*}$ or $\left|\delta_{k}\right| \sim \sqrt{\mu \Delta_{0}}$ at the rate (19) $\gamma_{\text {ind }} \approx \omega_{p e} w_{0} / 8>>v_{e} / 2$. The waves in the resonance zone, $\delta k_{\text {ind }} \sim r_{D}^{-1} \sqrt{\mu}$, grow until they become unstable at $\tau_{\text {ind }} \sim 10 / \gamma_{\text {ind }}$ and excite secondary waves. In saturation, the pumping $\sim \gamma_{\text {ind }}\left(W_{0}\right)$ is 
balanced by spectral transfer $\sim \gamma_{\text {ind }}\left(W_{1}\right)$, with $W_{1}=\int_{\delta k_{\text {ind }}} d k W_{\mathbf{k}}$. Therefore, the r.m.s. amplitude $A_{1}$ is of the order of the pump $\left|E_{0}\right|$. This process repeats itself in each further step creating a typical WT-cascade, which comprises $\sim \sqrt{\Delta_{0} / \mu}$ downshifted spectral peaks of the amplitude $\sim\left|E_{0}\right|$ and width $\sim \delta k_{\text {ind }}$, separated by $\Omega_{q} \sim \gamma_{\text {ind }}$. The wave energy is evenly distributed $\left(W_{k} \approx W_{0} / \delta k_{\text {ind }}\right.$ ) over the spectrum with the total energy density $W_{\text {ind }} \approx W_{0} \sqrt{\Delta_{0} / \mu}>>W_{0}$.

Eventually, plasmons accrue in the region, $k \leq k_{c} \approx r_{D}^{-1} w_{0} / 3 \mu^{1 / 2}<<k_{p}$, with the energy density $W_{c} \approx W_{0} w_{0} / 3 \mu \quad$ [Zakharov, 1984]. The plasmon condensate is subject to MI, so the WT and SLT regimes co-exist (cf. Figure 3.3). For $w_{c}>3 \mu$, the supersonic MI causes the energy outflow in collapsing cavities, $\sim \gamma_{m i}\left(W_{c}\right) W_{\text {cav }}^{\Delta}$, which balances the energy inflow, $\approx 2 \gamma_{\text {ind }} W_{0}$, giving $W_{\text {cav }}^{\Delta} \approx W_{0}$, much smaller than $W_{\text {cav }}^{0}$ at $\Delta_{0}=0$.

\subsubsection{Full-Wave Simulations of SLT at HAARP}

Figure 3.3 [Mishin et al., 2016] exemplifies the results of the Eliasson et al. [2012; 2015] fullwave 1-D modeling of SLT driven by a $3.2 \mathrm{MHz}$ ordinary wave with the input amplitude $E_{\text {in }}=1$ and $2 \mathrm{~V} / \mathrm{m}$ at $h_{i n}=200 \mathrm{~km}$ for vertical, $10.5^{\circ} \mathrm{S}$, and MZ injections at HAARP. Near $h_{0} \approx 231 \mathrm{~km}$, the gradient scale is $L_{n} \approx 43 \mathrm{~km}$ and the Airy scale length $l_{m z} \approx 2 l_{A} \approx 150 \mathrm{~m}$. The electron and ion temperatures are taken $T_{e 0}=2 T_{i 0}=0.4 \mathrm{eV}$, about two times higher than in the quiet ionosphere. The damping rates of Langmuir and ion acoustic waves are set to $10^{2}$ and $10^{3} \mathrm{~s}^{-1}$, respectively.

Shown are time vs. altitude color-coded logarithmic-scale plots of the vertical electric field $E_{z}$ (in $\left.\mathrm{V} / \mathrm{m}\right)$ and small-scale density variations $\delta n_{s}$ (in $\mathrm{m}^{-3}$ ). Clearly, Airy-like structures of $E_{z}$ are set up first. For $E_{i n}=1 \mathrm{~V} / \mathrm{m}$, the nonlinearity parameter in the first Airy maximum $w_{A}$ is 0.26 at vertical and 0.09 at $\mathrm{MZ}\left(\gg 4 v_{e} / \omega_{p e}\right)$, with

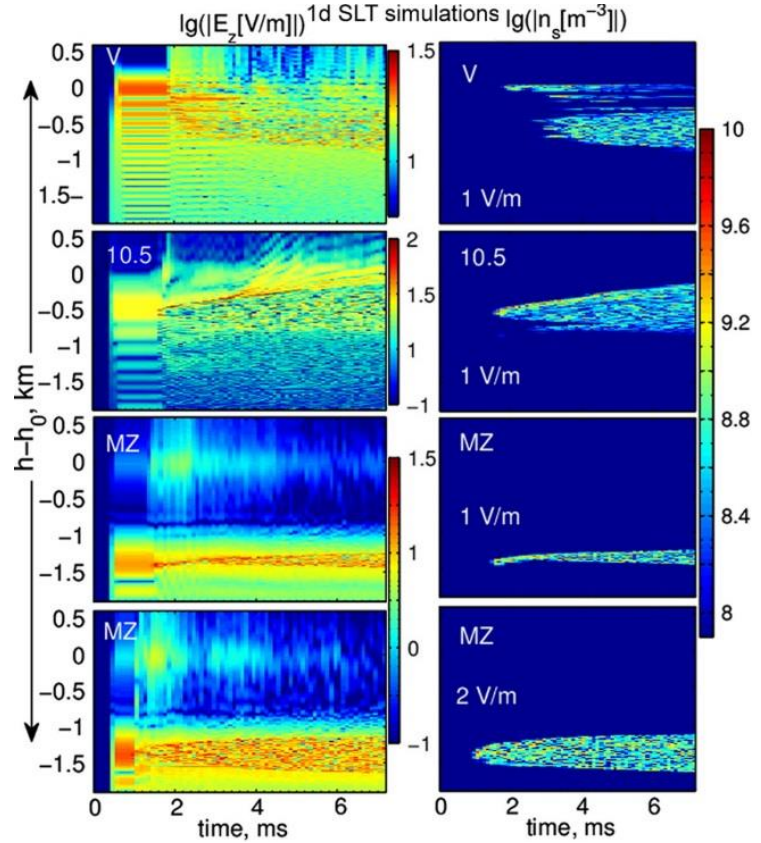

Figure 3.3. Results of $1 \mathrm{D}$ simulations of SLT at HAARP for different input amplitudes and injection angles, as indicated in plots [Mishin et al., 2016]. $\Delta_{m z} \approx 0.016\left(\Delta h_{m z} \approx-1.4 \mathrm{~km}\right)$. In about $1 \mathrm{~ms}$ the structures start breaking into small-scale turbulence. In the SLT region with the total energy density with $w_{\text {tot }} \sim 0.1-0.3$, solitary wave 
packets are trapped in density cavitons with the amplitudes $\delta n_{\text {cav }} / n_{0} \sim(0.3-3) 10^{-2}$ and widths $l_{a} \sim(10-20) r_{D}$ spaced apart by $\sim 50 r_{D}$. It is evident that $w_{t o t}>\delta n_{c a v} / n_{0}$, indicating the presence of non-trapped (free) waves, and that the SLT region is sandwiched between the WT regions with turbulent electric fields but without density cavitons. Note also the emergence of Z-mode waves around $h_{0}$, concurrent with the cavitating turbulence.

At vertical, the turbulent layer shifts gradually downward after saturation with the r.m.s. amplitude $\delta E_{\text {sat }}$ much smaller than for the initial stage (the SLT overshoot). The fast-time downward shift and the Z-mode waves are understood in terms of scattering off small-scale cavitons and ion oscillations (similar to (eq. 24)), i.e., $O+\delta n_{q} \rightarrow O^{\prime}$ Brillouin scatter and $O+\delta n_{q} \rightarrow Z$ conversion. The pump energy is scattered in the lower Airy maxima, thereby inhibiting SLT at $h_{A}$. The initial stage at $10.5^{\circ} \mathrm{S}$ and MZ appears the same as at vertical. At later times, the SLT layer at $10.5^{\circ} \mathrm{S}$ expands in both directions so its upper boundary reaches $h_{0}$ and the lower boundary overlaps with the $2^{\text {nd }}$ peak making the SLT altitudinal extent $l_{L T} \sim 5 l_{0}$. At MZ, the SLT layer remains near the $1^{\text {st }}$ peak for $E_{i n}=1 \mathrm{~V} / \mathrm{m}$, while for $2 \mathrm{~V} / \mathrm{m} l_{L T}$ increases with time up to $\sim 3 l_{0}$.

\subsection{PPI in the Upper Hybrid Layer (PPI $\mathbf{P}_{U / E B}^{O}$ )}

In the UH layer, the O-mode electric field with the amplitude (eq. 1) $E_{f s} \approx 0.5 p_{0}^{1 / 2} \mathrm{~V} / \mathrm{m}$ is perpendicular to $\mathbf{B}_{0}$ and the HF and LF partners are $\mathrm{UH}$ and LH waves. The ponderomotive force comprises two terms, $\mathbf{F}_{u h}+\mathbf{F}_{e b}$, with $\mathbf{F}_{u h}=\mathbf{F}_{p}$ (eq. 11) and $\nabla$ replaced by $\nabla_{\perp}$, while $\mathbf{F}_{e b}$ is significant for EB modes near the double resonance [Istomin and Leyser, 2005]. Likewise Langmuir waves, $\mathrm{UH} / \mathrm{EB}$ waves are excited via decay $\mathrm{PDI}_{U H / E B}^{O}, O \rightarrow U H / E B+L H$, four-wave $\mathrm{OTSI}_{U H}^{O}, O \rightarrow U H+\delta n_{\|}$, and four-wave second order decay $\operatorname{PDI}_{U H / E B}^{2 O}$ [Huang and Kuo, 1994; Istomin and Leyser, 1995; Kuo, 2013; 2015]. By virtue of the matching conditions, $\mathrm{PDI}_{U H}^{O}$ develops at $\omega_{0}>\omega_{u h r}$, i.e., below $h_{u h}$, regardless of the sign of $\delta_{u h r}=\omega_{u h r} / s \omega_{c e}-1$. However, $\operatorname{PDI}_{E B}^{O}$ can develop above and below $h_{u h}$ for $\delta_{u h r}>0$ and $<0$, respectively.

The corresponding wave equations can be derived similar to the Zakharov equations (14)-(15). Namely, the electron density (velocity) is split into LF ( $\delta N_{e}$ and $\mathbf{V}_{e}$ ) and HF ( $\delta n_{e}$ and $\mathbf{u}_{e}$ ) perturbations. That is, $n_{e}=n_{0}\left(1+\delta N_{e}+\delta n_{e}\right)$ and $\mathbf{v}_{e}=\mathbf{V}_{e}+\mathbf{u}_{e}$. The fast timescale is isolated using for UH waves $\phi=\operatorname{Re}\left(\Psi(\mathbf{r}, t) e^{-i \omega_{u h t} t}\right)$ and $\mathbf{E}_{u h}=-\nabla \phi$, with the envelopes $\Psi$ and $\mathbf{A}=-\nabla \Psi$. The pump amplitude due to the circular polarization is $E_{0}=E_{f s} / \sqrt{2}$ and $k_{0} \rightarrow 0$. Unmagnetized 
ions experience only a slow motion, with $n_{i}=n_{0}\left(1+\delta N_{i}(\Phi)\right)$ and $\mathbf{v}_{i}=\mathbf{V}_{i}(\Phi)$, with the slow potential $\Phi$ related to $\delta N_{e}$ via Poisson's equation $\delta N_{e}=\delta N_{i}+\Delta \Phi / 4 \pi n_{0} e$.

From the dispersion relation of UH waves (eq. 4) one can readily obtain the value of the UH wave vector required for $\mathrm{PDI}_{U H}^{O}$ to occur at a given altitude $h$ [Istomin and Leyser, 1995]

$$
\rho_{\perp}^{*}\left(h, \omega_{0}\right) \approx \frac{2}{3} \frac{s\left(s^{2}-4\right)}{s^{2}-1} \frac{f_{0}-f_{u h r}(h)-f_{l h r}(h)}{f_{c e}(h)}<0.5
$$

with $s$ defined by rounding up the $\omega_{u h r} / \omega_{c e}$ ratio to the nearest integer.

Figure 3.4 shows the threshold field for $\mathrm{PDI}_{U H / E B}^{O}$ for $s=5$ as a function of the frequency mismatch $f_{0}-f_{u h r}-f_{l h r}$. The instability growth rate is of the order of $v_{e}$ outside the forbidden band near the gyroresonance $\left|f_{0}-s f_{c e}\right| \sim f_{l h r} \sim 10$ $\mathrm{kHz}$. Otherwise, the instability is inhibited [Istomin and Leyser, 1995].

A second order four-wave process involves decay of the second harmonic pump oscillation, $2 \hat{k}_{0}=2\left(\mathbf{k}_{0}, \omega_{0}\right)$, into upshifted UH $\left(\hat{k}_{u h}\right)$ and down-shifted EB $\left(\hat{k}_{e b}\right)$ sidebands and a Istomin and Leyser [1995].

forced LH oscillation $\hat{k}_{l h}$ [Huang and Kuo, 1994; Hussein et al., 1998; Xi and Scales, 2001]. This process explains the well-documented SEE feature, the broad upshifted maximum (BUM) [Leyser, 2001; Grach et al., 2008]. In the dipole approximation, one gets $\mathbf{k}_{e b}=-\mathbf{q}_{l h}=-\mathbf{k}_{u h}$ and $\omega_{0}=\omega_{e b}+\Omega_{l h}^{*}=\omega_{u h}-\Omega_{l h}$ or $\omega_{u h} \approx 2 \omega_{0}-\omega_{e b} \approx 2 \omega_{0}-s \omega_{c e}+\delta \omega$ ("*" means complex conjugate). For $\Omega_{l h r}<\omega_{0}-s \omega_{c e} \leq 15 \Omega_{l h r}$ and $q r_{c e}<<1$, the instability develops at $E_{0}>E_{\text {bum }}=0.3-0.7 \mathrm{~V} / \mathrm{m}$ at the growth rate $\gamma_{\text {bum }} \sim(0.2-0.6) \Omega_{l h r}>>v_{e} / 2$.

If pump electric field is not strictly perpendicular to $\mathbf{B}_{0}$ and $T_{e}>3 T_{i}$, the Ion Acoustic (sound, S) parametric decay (IAPD) instability, $O \rightarrow U H / E B+S$, can develop [e.g., Sturman, 1974; Huang and Kuo, 1995]. Numerical simulations for $f_{0}<2 f_{c e}$, the off-perpendicular angle $\theta_{E}=22.9^{\circ}$, and $T_{e}=3 T_{i}$ show a broad IAPD spectral structure centered at $f_{0}-(0.3-0.5) \mathrm{kHz}$ [Samimi et al., 2013; 2014]. It has a number of similarities with the so-called downshifted peak in the SEE spectrum explored at EISCAT for $f_{0} \geq 3 f_{c e}$ [Leyser, 2001] and HAARP during highpower heating for $f_{0} \sim 3 f_{c e}$ [Mahmoudian et al., 2013].

\subsubsection{Upper Hybrid PPI}

As soon as the pump-excited or primary, $\omega_{1}=\omega_{0}-\Omega_{q}, \mathrm{UH} / \mathrm{EB}$ waves grow above the thermal noise, they become subjected to parametric interactions that excite the secondary, $\omega_{2}=\omega_{1}-\Omega_{q}$, 
waves, etc. [Zhou et al., 1994]. We consider a three-wave decay process ( $\left.\mathrm{PDI}_{U H}\right)$, $U H_{0} \rightarrow U H_{1}+L H$, taking account of oblique waves $k_{\|}<<k_{\perp}$ and $q_{\|}<<q_{\perp}$. Now, the electron quiver velocity in the first order in $\omega_{c e} / \omega_{p e}$ is

$$
\mathbf{u}_{e} \approx \operatorname{Re}\left(\frac{-i e}{m_{e} \omega_{p e}}\left(\mathbf{A}+\frac{i \omega_{c e}}{\omega_{p e}}\left[\mathbf{e}_{z}, \mathbf{A}\right]\right) e^{-i \omega_{p e} t}\right)
$$

$\left(\mathbf{e}_{z} \| \mathbf{B}_{0}\right)$, and the ponderomotive force becomes [Volokitin and Kraft, 2005]

$$
\begin{aligned}
\mathbf{F}_{p} & =e \nabla \Phi_{p}-e \delta \mathbf{E}_{p}, \\
\Phi_{p} & =-\frac{1}{16 \pi e n_{0}}\left(|\mathbf{A}|^{2}+\frac{i \omega_{c e}}{\omega_{p e}}\left[\mathbf{A}, \mathbf{A}^{*}\right]_{z}\right) \\
\delta \mathbf{E}_{p} & =\frac{i \omega_{c e}\left[\mathbf{e}_{z},\left(\mathbf{A}^{*} \nabla\right) \mathbf{A}-(\mathbf{A} \nabla) \mathbf{A}^{*}\right]}{16 \pi e n_{0} \omega_{p e}} \equiv \frac{i \omega_{c e}}{\omega_{p e}}\left[\mathbf{e}_{z}, \mathbf{K}\right]
\end{aligned}
$$

Here $\left[\mathbf{A}, \mathbf{A}^{*}\right]_{z} \equiv\left[\mathbf{A}, \mathbf{A}^{*}\right] \cdot \mathbf{e}_{z}$ is the vector nonlinearity term, non-existent in isotropic plasma.

That makes the UH turbulence essentially three dimensional. At $q_{z}<<\mu^{1 / 2} q$ and $\Omega_{q} \approx \Omega_{l h r}$, the decay growth rate is [Volokitin and Kraft, 2005]

$$
\gamma_{u h} \approx q r_{c e} \cos \alpha \sqrt{\omega_{p e} \Omega_{l h r} w_{0} / 2}
$$

where $\alpha$ is the angle between $\mathbf{k}_{0}$ and $\mathbf{k}_{1}$. The linear damping defines the threshold $w_{u h} \approx 4 v_{e}^{2} /\left(q^{2} r_{c e}^{2} \omega_{p e} \omega_{l h r}\right) \sim 10^{-5}$ or $E_{0}>E_{u h} \sim 0.08 \mathrm{~V} / \mathrm{m} \quad$ [cf. Zhou et al., 1994]. For $\Omega_{q} \approx \omega_{c e} q_{z} / q_{\perp}>\mu^{1 / 2} \omega_{p e}$ and $\left|\left[\mathbf{k}_{0}, \mathbf{q}\right]_{z}\right|>>q^{2} \omega_{c e} / 2 \omega_{p e}$, the growth rate becomes

$$
\gamma_{d o} \approx k_{0} r_{D} \sin (2 \alpha) \sqrt{\omega_{p e} \Omega_{q} w_{0} / 4}
$$

at $w_{0} \sin (2 \alpha)>w_{d o} \approx 2 v_{e}^{2} /\left(k_{0}^{2} r_{D}^{2} \omega_{p e} \Omega_{q}\right) \sim 10^{-4}$ or $E_{0}>E_{d o} \sim 0.5 \mathrm{~V} / \mathrm{m}$.

It is assumed that $\left|\omega_{0} / s \omega_{c e}-1\right|>\mu^{1 / 2}, q r_{c e}<1 / 4$, and $q_{\|} v_{T e}>>\Omega_{q}>>q v_{T i}$. Otherwise, the threshold greatly increases due to strong cyclotron and Landau damping of UH and LH waves, respectively. The important corollary is that $\mathrm{PDI}{ }_{U H}$ is inhibited in the forbidden band $\left|\omega_{0}-s \omega_{c e}\right| \leq \Omega_{l h r}$. The saturation of PDI ${ }_{U H}$ proceeds along the same weak-turbulence scenario as for $\mathrm{PDI}_{L}$, leading to the cascading pattern of downshifted spectral peaks of $\delta E_{r m s} \sim\left|\mathbf{E}_{0}\right|$, spaced apart by $\delta \omega_{u h} \sim \Omega_{q}$. The total UH wave energy density can be estimated as $W_{u h}^{*} \sim \Lambda W_{0}$ with $\Lambda \sim\left|\omega_{0}-s \omega_{c e}\right| / \Omega_{q}$.

At $\Omega_{q} \approx \Omega_{l h r}$, the resulting UH spectrum after conversion into electromagnetic emission forms the well-known family of downshifted maxima (DM) [e.g., Leyser, 2001]. For $\Omega_{q} \sim \omega_{c e} q_{z} / q_{\perp}$, the downshifted spectrum extends to tens of $\Omega_{l h r}$ somewhat similar to the 
recently discovered broad downshifted emission (BDE) [Grach et al., 2016]. This process can be important for saturation of the slow thermal parametric instability (section 3.6.3) as it limits the energy of the primary waves.

\subsubsection{Langmuir Turbulence in the UH Layer}

At high powers, $w_{0}>w_{l o} \sim 0.1\left(\omega_{c e} / \omega_{p e}\right) \mu^{1 / 2} \sim 10^{-4}>>v_{e} / \omega_{p e}$ or $E_{0}=E_{f s}>E_{l o} \sim 0.5 \mathrm{~V} / \mathrm{m}$, the four-wave modified decay instability (or OTSI ${ }_{L o}^{U H}$ ), $U H \rightarrow L o+\delta n_{\|}$, is also excited. It generates short-scale oblique red- and blue-shifted Lo-sidebands with $k_{l o} \sim 3^{-1 / 2} r_{c e}^{-1}$ and $\Delta \omega_{ \pm} \approx \pm \omega_{l h r} k_{0} r_{c e} \sqrt{T_{i} / T_{e}}$ at the growth rate $\gamma_{l} \sim 0.1 \gamma_{m i}$ (eq. 22) [Sturman, 1974; Kuo et al., 1997]. Further development is the same as that for the plasma resonance with the large frequency mismatch (section 3.4.2). That is, the small-scale plasmons are transferred into large scales via induced scattering on ions, thus creating consecutive red-shifted peaks of the widths $\sim \delta k_{\text {ind }}$ and the energy density $W_{l o}=\int_{\delta k_{\text {ind }}} W_{k} d k \sim 0.1 w_{0}^{-1 / 3} W_{0}$.

The energy is evenly distributed over the spectrum $\left(W_{k} \approx W_{l o} / \delta k_{\text {ind }}\right)$, with the total energy density $W_{\text {ind }} \sim W_{l o}\left(\omega_{c e} / \omega_{p e}\right) \mu^{-1 / 2}>>W_{0}$. Eventually, plasmons accrue in the condensate region, $k \leq k_{c} \approx r_{D}^{-1} w_{l o} / 2 \mu^{1 / 2}<<k_{l o}$, with the energy density $W_{c} \approx W_{l o} k_{c} / \delta k_{\text {ind }} \approx 3 k_{c}^{2} r_{D}^{2} n T_{e}$. The plasmon condensate is subject to MI and concomitant collapse, so the UH-pumped WT and SLT regimes co-exist. Balancing the energy inflow, $\sim \gamma_{i n d} W_{l o}$, by the outflow in collapsing cavities, $\sim \gamma_{m i}\left(W_{c}\right) W_{\text {cav }}^{L o}$, gives $W_{\text {cav }}^{L o} \sim W_{l o} / 2<<W_{c}$.

This mechanism was instrumental in explaining airglow in the underdense ionosphere at low powers for various frequencies [Mishin et al., 2004; 2005a]. At high powers, during one experiment with $f_{0}=4.5 \mathrm{MHz}$ two layers of enhanced ion acoustic radar backscatter have been observed, likely, near the plasma resonance and near $h_{u h}$ (section 4.6)]. Recently, Grach et al. [2015] reported on a novel downshifted feature in the SEE spectrum between the DM and pump, symmetric to the well-known upshifted maximum. It is dubbed the intermediate downshifted maximum (IDM) and explained in terms of OTSI ${ }_{L o}^{U H}$. It should be noted that OTSI ${ }_{L o}^{U H}$ is facilitated at $\omega_{0}>s \omega_{c e}(s \geq 3)$ [Kuo et al., 1997] and hence its overall effect should be greater above the gyroresonance. This is consistent with the observations of greater artificial ionization production (section 4.6).

\subsubsection{Lower Hybrid PPI}

Excited LH waves can serve as a "high-frequency" pump in the lower hybrid range $\left(\mathrm{PPI}_{L H}\right)$. Let us consider coupling of a monochromatic LH pump wave, $\phi=\operatorname{Re}\left(\Psi(\mathbf{r}, t) e^{-i \Omega_{l h} t}\right)$ with $\mathbf{E}=-\nabla \phi$ and $\mathbf{A}=-\nabla \Psi$, at $\Omega_{k_{0}} \approx \Omega_{l h r}$ and $\mathbf{k}_{0}=k_{0} \mathbf{e}_{x}$, to slow $\left(\Omega<<\Omega_{l h r}\right)$ perturbations $\delta N_{e, i}$ 
and $\Phi$. Unmagnetized ion motion under the ambipolar (second order of magnitude) electric field maintains quasi-neutrality, $\delta N_{e} \approx \delta N_{i}=\delta N_{s}$. Now, electrons are magnetized also in the pump and sideband waves. Their drift $\mathbf{V}_{E} \approx\left[\mathbf{e}_{z}, c \nabla \phi / B_{0}\right]$ in the presence of LF density variations $\delta N_{e}$ yields in the second order electron density variation $\partial \delta n_{e}^{(2)} / \partial t \sim\left[\nabla \phi, \nabla \delta N_{e}\right]_{z}$. Accordingly, the ponderomotive force

$$
\mathbf{F}_{p l h}=-m_{e} \overline{\left(\mathbf{v}_{e} \nabla\right) \mathbf{v}_{e, z}} \approx-i \frac{\omega_{p e}^{2}}{\omega_{c e} \Omega_{l h r}} \nabla_{z} \frac{\left[\mathbf{A} \times \mathbf{A}^{*}\right]_{z}}{16 \pi n_{0}}
$$

contains the vector nonlinearity similar to $\mathrm{PDI}_{U H}$.

At $\mathbf{q} \perp \mathbf{k}_{0}$, one has for the red- and blue-shifted sidebands $\left|\mathbf{k}_{ \pm}\right|=\sqrt{q^{2}+k_{0}^{2}}$ and from (eq. 7) $\delta_{\mathbf{k}_{ \pm}}-\delta_{\mathbf{k}_{0}}=\frac{1}{2}\left(q^{2} r_{c^{*}}{ }^{2}+q_{z}^{2} / \mu k_{+}^{2}\right) \equiv \delta_{0}$. Above the threshold $w_{t h}=2 \mu \delta_{0}\left(1+q^{2} / k_{0}^{2}\right), \quad$ the modulational instability has the growth rate

$$
\gamma_{l h}^{m i}=\Omega_{l h r} \delta_{0} \sqrt{w_{0} / w_{t h}-1}
$$

with $w_{k}=\left(\omega_{p e}^{2} / \omega_{c e}^{2}\right)\left|\mathbf{E}_{\mathbf{k}}\right|^{2} / 8 \pi n T_{e}$. The Landau and collisional damping can be neglected at $\min \left(\Omega_{l h r} / k v_{T i}, \Omega_{l h r} / k_{z} v_{T e}\right)>>1$ and $v_{e}<<\Omega_{l h r}$.

The fluid approach gives an accurate account for nonlinear interactions of a narrowband LH spectrum, $\Omega_{0} \sim \Omega_{l h r}$ and $\Delta \Omega<\left|d \ln W_{\mathbf{k}} / d t\right|$. For a broadband LH spectrum, the induced scattering (nonlinear Landau damping) by thermal electrons is derived from the kinetic equation that gives the growth rate

$$
\gamma_{l h}^{i n d} \approx \frac{\omega_{c e}^{2}}{2 \Omega_{l h}} w_{0}>>v_{e}
$$

The maximum of $\gamma_{l h}^{\text {ind }}$ is reached at $\Omega_{\mathrm{\kappa}} \approx \Omega_{0}-\left(k_{0 z}-q_{z}\right) v_{T e}$. At $k^{2} r_{c^{*}}^{2}<k_{z}^{2} / \mu k^{2}$, the decrease of $\Omega_{\mathbf{k}}$ by $\delta \Omega_{k} \sim \Omega_{l h r} k r_{c *}<<\Omega_{0}$ at each spectral step is due to increasing $k$. The ultimate "cascading" spectrum between $\Omega_{0}$ and $\Omega_{l h r}$ consists of spectral peaks of the r.m.s. amplitude $E_{l h} \approx E_{0}$. The induced scattering of LH waves can result in accumulation of the LH energy near $\Omega_{l h r}$ and subsequent LH modulational instability (eq.31) creating cigar-shape, $l_{\perp} / l_{\|} \sim w_{l h}^{1 / 2} \omega_{p e} /$ $\omega_{c e}$, LH cavitons [Musher et al., 1978; Shapiro and Shevchenko, 1984]. Kosch et al. [2007a] have invoked LH cavitons to interpret weak backscatter from the Kodiak radar at $f_{0}<2 f_{c e}$ when the thermal parametric instability (section 3.6.3) is inhibited.

\subsection{Nonlinear Thermal Effects}

Thus far, we considered the ambient plasma parameters, $n_{0}$ and $T_{e 0}=T_{0}=(1.5-2) T_{i 0}$, as given. This assumption is well justified for the ponderomotive instabilities with timescales, $\tau_{p p i} \sim\left(\mu \omega_{p e}\right)^{-1}$, much shorter than the plasma heating $\left(\tau_{T}\right)$ and diffusion $\left(\tau_{D}\right)$ times. In general, 
heating by the pump and plasma waves in the turbulence region results in the increase of the electron temperature, $T_{e}=T_{0}\left(1+\delta T_{e}\right)$, and pressure

$$
\delta p_{e}=p_{e} / n_{0} T_{0}-1=\delta T_{e}+\delta N\left(1+\delta T_{e}\right)
$$

where $\delta N=n_{e} / n_{0}-1$. While electrons are forced out from the heated region, the emerging electric field pulls ions to preserve charge neutrality. Thereby, the density in this region is further depleted. This affects the propagation of waves and leads to specific thermal instabilities. Before discussing these instabilities let us outline the electron heating process.

\subsubsection{Electron Heating and Thermal Flux}

The perturbed electron temperature is evaluated from the energy balance

$$
\begin{aligned}
& \frac{3}{2} n_{e} T_{0}\left(\frac{\partial}{\partial t}+v_{e} \delta_{i l}\right) \delta T_{e} \approx v_{e} W_{t o t}-\nabla \mathbf{q}_{T} \\
& q_{T}=-n_{e} \kappa_{\| e} \nabla T_{e} \approx-6 n_{e} T_{e} v_{T e} K_{T} \operatorname{sign}\left(\nabla_{\|} T_{e}\right)
\end{aligned}
$$

Here $v_{e} W_{\text {tot }}$ is the collisional (Ohmic) heating rate by the pump and plasma waves, $q_{T}$ is the electron heat flux $\left(\| \mathbf{B}_{0}\right), \lambda_{e}=v_{T_{e}} / v_{e}$ is the mean free path of thermal electrons, $\kappa_{\| e} \approx 6 v_{e} \lambda_{e}^{2}$ is the parallel electron thermal conductivity, and $K_{T}=\lambda_{e}\left|\nabla_{\|} \ln T_{e}\right|$ is the Knudsen number. The coefficient of inelastic losses $\delta_{i l}\left(T_{e}\right)$ at $\sim 200 \mathrm{~km}$ varies in the range $\approx(1-5) \times 10^{-3}$ for $T_{e} \approx 0.15$ $-0.4 \mathrm{eV}$ [e.g., Gurevich, 1978]. For estimates, we assume $T_{0}=0.2 \mathrm{eV}$ and $v_{e}=300 \mathrm{~s}^{-1}$, as typical for the F-region ionosphere. The thermal conduction balances $v_{e} W_{t o t}$ at $K_{T}=K_{c} \approx\left(w_{t o t} / 6\right)^{1 / 2}$. At $K_{T}<K_{c}$ and $w_{t o t}=10^{-3}$ to $10^{-2}$, inelastic losses dominate, and equation (34) yields $\delta T_{e}^{(i l)}=2 w_{\text {tot }} / 3 \delta_{i l}\left(T_{e}^{(i l)}\right) \approx 0.5$ to $0.8\left(T_{e}^{(i l)} \approx 0.3\right.$ to $\left.0.36 \mathrm{eV}\right)$ in $\tau_{T}=\tau_{i l} \approx 1 / \nu_{e} \delta_{i l} \sim 1-10 \mathrm{~s}$. The steady state is achieved at $K_{T}=K_{\infty} \sim K_{c}\left(T_{e}^{(i l)}\right)$.

Accounting for collisionless heating decreases $\tau_{T}$ and increases $T_{e}$. In the upper hybrid layer, for example, the induced scattering of $\mathrm{LH}$ waves causes the LH wave energy flow to the bulk electrons at a rate $Q_{l h}^{\text {ind }} \sim \gamma_{l h}^{\text {ind }} W_{l h}$ [Musher et al., 1978]. Vlasov simulations of the O-mode interaction with density striations show parametric excitation of UH and EB wave turbulence and stochastic heating of bulk electrons up to 0.4-0.5 eV [Najmi et al., 2016].

Another deviation from the collisional approach is brought about by the thermal flux-driven instability in the heated region for $K_{T}>K_{w} \approx 2\left(\mu^{1 / 2}+\gamma_{i o n}(k)\right)$, where $\gamma_{i o n}$ is the ion Landau damping [e.g., Forslund, 1970; Mishin, 1974]. For $\lambda_{e} \geq 1 \mathrm{~km}$ (above $200 \mathrm{~km}$ ), this condition is satisfied for $\left|\nabla_{\|} \ln T_{e}\right|^{-1}<100 \mathrm{~km}$ and $T_{e}>3 T_{i}$. Far beyond the threshold, the saturated ion-sound spectrum, with the total energy density $W_{s} \sim\left(10^{-3}-10^{-2}\right) n_{0} T_{e}$, maximizes between 
$k_{s} \sim(0.2-0.3) / r_{D}$ and $\sim 1 / r_{D}$ [Bychenkov et al., 1988]. Notably, waves at $k<0.5 / r_{D}$ are highly oblique (angles $\theta$ up to $\sim \pi / 2$ ) and more field-aligned otherwise.

Electron scattering by enhanced waves, $v_{w}>>v_{e}$, reduces the mean free path $\lambda_{w}=v_{T e} / v_{w}<<\lambda_{e}$. Therefore, the parallel $\kappa_{\| e}$ thermal conductivity decreases by $\sim v_{w} / v_{e}>>1$, while $\kappa_{\perp e}$ increases. Heuristically, $\mathbf{q}_{T}$ can be presented as

$$
\mathbf{q}_{e w}=-n_{e} v_{e w}\left(r_{c e}^{2} \nabla_{\perp}+\lambda_{e w}^{2} \nabla_{\|}\right) T_{e}
$$

where $v_{e w}=v_{e}$ and $\lambda_{e w}=\lambda_{e}$ at $K_{T}<K_{w}$, while $v_{e w}=v_{w}$ and $\lambda_{e w}=\lambda_{w}$ at $K_{T}>K_{w}$. As a result, the parallel heat flux is reduced to $q_{\text {turb }}=\kappa_{\text {turb }} n_{e} T_{e} v_{T e}$, with $\kappa_{\text {turb }}$ in the range from $7.5 \mu^{0.5}$ to $\mu^{0.25}$ [Mishin, 1974; Bychenkov et al., 1988].

While waves propagate along $\nabla_{\|} T_{e}$, i.e., downward above and upward below the temperature peak, their wavenumbers decrease and may become detectable by UHF radars. Apparently, greater values of $K_{T}$ at higher altitudes favor the instability above the $T_{e}$ peak and thereby the upshifted shoulder in the ion acoustic Doppler spectra (section 4.6).

\subsubsection{Thermal Self-Focusing Instability (TSFI)}

TSFI develops near the plasma resonance [Gurevich, 1978]. Its positive-feedback loop, $\delta E_{H F} \rightarrow \delta N \rightarrow \delta E_{H F}$, is similar to the modulational instability of Langmuir waves, except for the cause of $\delta N$ (thermal, $\delta p_{e}$, vs. strictional, $\delta p_{h f}$ ). Indeed, as follows from the dispersion relation $\omega_{k} \approx \omega_{p e}+k^{2} c^{2} / 2 \omega_{p e}$, photons accumulate in large-scale $\left(L_{\perp}>>c / \omega_{p e}\right)$ density depletions $\delta N<0$. This leads to the increase of $\delta p_{e}$ and further deepening of the initial depletion, thereby closing the loop. Evidently, the TSFI development is facilitated by pre-existing large-scale, $L_{\perp}>c / \omega_{p 0}$, depletions. As a result of TSFI, a uniform $(\perp \mathbf{k}) \mathrm{HF}$ radio beam splits into filaments with the enhanced HF power. Their transverse size $\lambda_{\perp} \sim \frac{c}{\omega_{p 0}}|\delta N|^{-1 / 2}>>2 \pi / k_{0}$. Since $|\delta N|<<1$, the minimum size is much greater than the plasma skin depth $c / \omega_{p 0} \approx 5 / n_{0}^{1 / 2} \mathrm{~km}$. According to the numerical simulations by Guzdar et al. [1998] km-scale filaments grow initially and in $\sim 10 \mathrm{~s}$ break into smaller (10s to 100s meters) scale sizes. Kosch et al. [2007b] invoked TSFI to explain the temporal evolution of pump beam self-focusing in the low-power experiment, as did Mishin and Pedersen [2011] for creation and quenching of the artificial ionization in the high-power HAARP experiments in March 2009 (section 4.6).

\subsubsection{Thermal Parametric Instability (TPI)}

Another nonlinear thermal instability, however, with $\lambda_{\perp}<c / f_{0}$ develops in the upper hybrid layer with the growth time $\tau_{t p i}$ of the order of a few seconds. It is called Thermal Parametric 
Instability (TPI), which originates from plasma polarization $\rho_{e}$ caused by an O-mode wave propagating along $\mathbf{B}_{0}$ in the presence of field-aligned density irregularities $\delta N\left(\mathbf{r}_{\perp}\right)=n_{e}\left(\mathbf{r}_{\perp}\right) / n_{0}-1$

$$
\rho_{e}=\nabla_{\perp} \frac{\omega_{p e}^{2}}{\left(\omega_{0}+\omega_{c e}\right)} \frac{\mathbf{E}_{0}}{4 \pi \omega_{0}} \propto\left|\mathbf{E}_{0}\right| \nabla_{\perp} \delta N e^{-i \omega_{0} t}
$$

At $h \leq h_{u h}$, the polarization charge oscillates with $\omega_{0} \geq \omega_{u h r}$. Thus, UH waves can get into resonance $\omega_{u h}\left(\mathbf{k}_{\perp}\right)=\omega_{0}$ and grow at the expense of the O-mode pump. This is simply $O+\delta N \rightarrow U H$ conversion.

Anisotropic heat and plasma transport makes irregularities strongly field-aligned, i.e., $l_{\|}=\left.\left|\nabla_{\|} \ln \right| \delta N\right|^{-1}>>c / f_{0}>l_{\perp}=\left.\left|\nabla_{\perp} \ln \right| \delta N\right|^{-1}$. Field-aligned, cigar-shaped density cavities are termed striations. Trapping UH waves inside striations leads to enhanced Ohmic heating in the tenuous regions. This enhances the pressure imbalance and further deepens the initial depletion, thus closing the positive feedback loop.

TPI in a homogeneous plasma can be derived using the same approach as for PDI ${ }_{U H}$, merely replacing $\delta p_{h f}$ by $\delta p_{e}$ and including slow processes of heating (see eq. 34-36) and ambipolar diffusion

$$
\frac{\partial}{\partial t} \delta N=\left(D_{\perp} \Delta_{\perp}+D_{\|} \Delta_{\|}\right)\left(\delta N+\kappa_{T} \delta T_{e}\right)
$$

Here $\kappa_{T} \sim 1 \quad$ is the thermodiffusion coefficient, $D_{\|}=\left(1+T_{e} / T_{i}\right) v_{T i}^{2} / v_{i} \quad$ and $D_{\perp}=\left(1+T_{i} / T_{e}\right) \nu_{e w} r_{c e}^{2}$ are parallel and perpendicular diffusivities at $\mu v_{e w}<<v_{i}$ (the ion collision frequency) and $l_{\|}>>\lambda_{i}=v_{T i} / v_{i}$. Ionization and recombination processes are neglected.

The losses due to plasma diffusion, $\tau_{D}^{-1}=\left(l_{\perp}^{-2} D_{\perp}+l_{\|}^{-2} D_{\|}\right), \quad$ and heat, $\tau_{T}^{-1}=v_{e} \delta_{i l}+\frac{2}{3}\left(l_{\|}^{-2} \kappa_{\|}+l_{\perp}^{-2} \kappa_{\perp}\right)$, define the TPI threshold [Grach et al., 1981; 2016]

$$
w_{t p i}=\delta_{i l} \frac{v_{e w}}{2 \omega_{0}}\left(\frac{v_{e}}{v_{e w}}+l_{\|}^{-2} L_{T}^{2}+l_{\perp}^{-2} L_{T \perp}^{2}\right)
$$

Here $L_{T}=\lambda_{e} \delta_{i l}^{-1 / 2}$ (the thermal conduction length) and $L_{T \perp}=r_{c e} \delta_{i l}^{-1 / 2}$. Clearly, for $T_{e}$ to grow, heating must overcome the losses. Pre-existing short-scale $\left(l_{\perp}<c / f_{0}\right)$ striations $\left|\delta N_{0}\right|>2^{1 / 2} v_{e} / \omega_{0}$ facilitate the TPI development lowering the threshold by a factor of $\omega_{0}\left|\delta N_{0}\right| / 2^{3 / 2} v_{e}$.

Just above the threshold, TPI results in a soliton-like structure across $\mathbf{B}_{0}$, comprising of striations with trapped UH waves ("thermal cavitons") [Burinskaya and Volokitin, 1981; Dysthe et al., 1982]. For $\nabla_{\|}=l_{\|}^{-1}=0$, the characteristic scale size of striations $\left(l_{s t}\right)$ can be found as 
follows. In equilibrium, we have $\delta N_{s t} \approx-\delta T_{e} \approx-2 v_{e w} \tau_{T} w_{t p i} / 3$. Then, the trapping condition $\left|\delta N_{s t}\right| \sim 3 r_{D}^{2} / l_{s t}^{2}(9)$ gives $l_{s t} \sim r_{D}\left(2 v_{e w} \tau_{T} w_{t p i}\right)^{-1 / 2}>>r_{c e}$.

At $w_{0} \gg w_{t p i}$, a wideband UH spectrum around $2 \delta_{\mathbf{k}}=\left(3 \omega_{p e} k^{2} r_{D}^{2}+\omega_{c e}^{2} \mathbf{k}_{\perp}^{2} / \omega_{p e} k^{2}\right)=v_{e w}$ is excited in a wide cone $\Delta \alpha \sim 1$ around $\mathbf{E}_{0}$. Its maximum growth rate

$$
\gamma_{t p i} \approx \sqrt{\left(2 \omega_{p e} / 3 \tau_{D}\right) w_{0}}
$$

has to be smaller than $v_{e}$ or $w_{0}<3 \tau_{D} v_{e}^{2} / 2 \omega_{p e}$. Otherwise, a three-wave decay ( $\mathrm{PDI}_{U H / E B}^{O}$ ) with the greater threshold should be considered.

The threshold of TPI in the F region can be derived using V. Trakhtengerts's energy balance considerations [Grach et al., 2016]. The scale length of the TPI excitation (heating) region, $L_{t p i}<<L_{n}$, is defined by the condition of synchronism between the pump and UH waves near the matching altitude $z_{m}$

$$
L_{t p i} \approx\left|\frac{d k_{z}}{d z}\right|_{z_{m}}^{-1 / 2} \approx\left|\frac{d \varepsilon / d k_{z}}{d \varepsilon / d z}\right|_{z_{m}}^{1 / 2}
$$

where $\varepsilon(\omega, \mathbf{k})$ is the scalar dielectric constant.

The lifetime of the excited waves in the excitation region, $\tau_{\|} \approx L_{t p i} / v_{g z}<<v_{e}^{-1}$, is defined by the group velocity $v_{g z}=\left|\left(d \varepsilon / d k_{z}\right) /(d \varepsilon / d \omega)\right|_{z_{m}}$ rather than the collisional damping $\sim v_{e}^{-1}$. For $\omega_{0}>>\omega_{c e}$ and far from the double resonance, we have $|d \varepsilon / d \omega| \approx 2 / \omega_{p e}$ and $|d \varepsilon / d z|_{z_{m}} \approx L_{n}^{-1}$. At $L_{t p i}<<L_{T}$, the heat will be distributed along $\mathbf{B}_{0}$ over the thermal conduction length $L_{T}$. The overall weakening of the thermal feedback process in non-uniform plasma is thus given by the product $\beta_{\text {non }} \sim\left(v_{e} \cdot \tau_{\|}\right) L_{t p i} / L_{T} \sim 2 v_{e} L_{n} / \omega_{p e} L_{T}<<1$.

Dividing the r.h.s. of (eq. 39) by $\beta_{\text {non }}$ yields at $\omega_{\mathbf{k}}=-v_{e} / 2$ the estimate of the TPI threshold

$$
w_{t p i}^{(\text {non })} \sim L_{T}\left(1+l_{\perp}^{-2} L_{T \perp}^{2}\right)^{1 / 2} / 2 L_{n}>>w_{t p i}
$$

At $w_{0}>>w_{t p i}^{(n o n)}$ the growth rate maximizes at $L_{T \perp} / l_{\perp} \sim w_{0} / w_{t p i}^{(n o n)}$ [Grach et al., 2016] and

$$
\gamma_{\text {tpi }}^{(\text {non })} \approx \delta_{i l} v_{e}\left(w_{0} / w_{t p i}^{(n o n)}\right)^{2}<<v_{e}
$$

It is so far implied that collisionless damping is negligible and $\omega_{0}$ is far from the double resonance. Near the double resonance TPI is inhibited due to strong coupling with EB modes that are not trapped in striations and do not contribute to TPI [Mjølhus, 1993]. Besides, the existence domain of transverse UH waves decreases both in real and $k$ space [e.g., Grach et al., 2016]. In particular, at $\omega_{0}<s \omega_{c e}$ the $k$-space UH domain $\left(\partial \omega / \partial k_{\perp}>0\right)$ shrinks rapidly while $\omega_{0} \rightarrow s \omega_{c e}$ for $s \geq 3$ (see Figure 3.1). Suppression of the striations near gyroharmonics is well documented 
[Stubbe, 1996; Honary et al., 1999; Hysell et al., 2010].

Similarly, the TPI efficiency is reduced for $f_{0} \leq 2 f_{c e}$ [Mishin et al., 2005b; Kosch et al., 2007a] as EB modes in the region $f_{u h r} \leq 2 f_{c e}$ at $k_{\|} \rightarrow 0$ are not trapped by striations. For $k_{\|}<<r_{c e}^{-1}$, trapping is limited to waves with relatively small $k_{\perp}<5 k_{\|}$[e.g., Grach, 1979; Hysell et al., 2010]. Such oblique waves are unable to attain short transverse wavelengths, thereby deep striations are not formed. This is consistent with the observations (section 4.6).

At the stage of developed TPI, while UH waves and striations grow, electron heating by UH waves becomes more efficient than heating from the pump only. That further intensifies the density perturbation and energy transfer into UH waves so the process acquires the character of explosive instability, $|\delta N| \propto\left(t_{\infty}-t\right)^{-1}$, termed the resonant instability. The latter is the basis of theory of anomalous absorption due to multiple scattering off striations [e.g., Gurevich, 2007]. However, its applicability at high powers can be severely limited by the development of the PPI processes (section 3.5) [Grach et al., 2016; Mishin et al., 2016].

\subsection{Electron Acceleration}

As discussed above, Langmuir collapse leads to the wave energy burnout due to absorption by plasma electrons. That results in a non-Maxwellian high-energy tail distribution function (TDF) $F_{t}(\varepsilon)$ described by a power-law function

$$
F_{t}(\varepsilon)=C_{a} \cdot \varepsilon^{-b} \text { at } \varepsilon_{\max } \geq \varepsilon \geq \varepsilon_{\min } \gg T_{e}
$$

Numerical modeling of 1-D electron acceleration in a Maxwellian $\left(F_{0}=F_{M}\right)$ homogeneous plasma yields $b \approx 1.5, \varepsilon_{\min }^{(M)} / T_{e} \approx 10-20$, and the tail density $n_{t}=\alpha_{t} n \approx\left(10^{-4}-10^{-3}\right) n$ [Galeevet al., 1983; Wang et al., 1997]. The value of $n_{t}$ is mainly defined by the cross-link condition, $F_{t}\left(\varepsilon_{\min }\right)=F_{0}\left(\varepsilon_{\min }\right)$. In the presence of the ambient ("seed") suprathermal population $F_{s}(\varepsilon)$ with $F_{s}\left(\varepsilon_{\min }^{(M)}\right) \gg F_{M}\left(\varepsilon_{\min }^{(M)}\right)$, the minimum energy $\varepsilon_{\min }^{(s)}$ greatly exceeds $\varepsilon_{\min }^{(M)}$ [Mishin and Telegin, 1986]. As a result, many more energetic electrons can be accelerated from photoelectrons during daytime than would be in night [Mishin et al., 2004].

Figure 3.5 exemplifies the Eliasson et al. [2012; 2015] 1D modeling of the accelerated TDF, $F_{t}(\varepsilon)=F_{t}(u) d u / d \varepsilon$, for MZ injections and time-vs-altitude plots of the artificial plasma density in $\mathrm{cm}^{-3}$ for $\mathrm{V}$ and $10.5^{\circ} \mathrm{S}$ injections at $E_{\text {in }}=1,1.5$, and $2 \mathrm{~V} / \mathrm{m}$. Overall, the main part of the TDF can be fitted by a power law such as $F_{t}(\varepsilon)$ (eq. 43) but with $b$ depending on $E_{i n}$ and $T_{e}$. This is easily understood as the maximum energy $\varepsilon_{\max }$ depends on the transit time $\tau_{a} \sim l_{L T} / \varepsilon_{\max }^{1 / 2}$ with $l_{L T}$ increasing with $E_{i n}$, while the tail density $n_{t}$ is mainly defined by the cross-link condition at

$\varepsilon_{\min }$. These factors and the input value of $T_{e}$ lead to considerable differences in the TDF. In 
particular, the TDF at $10.5^{\circ} \mathrm{S}$ is more enhanced than at vertical due to greater $l_{L T}$. Accordingly, the plots of the plasma density show faster ionization by the accelerated electrons and concomitant descent of artificial plasma at $10.5^{\circ} \mathrm{S}$ than at vertical.

A remark regarding a quantitative comparison of the 1-D SLT simulation results against data is in order. Although at $w_{0}<<\omega_{c e}^{2} / \omega_{p e}^{2}$ the modulational instability is excited in a narrow cone around $\mathbf{B}_{0}$, pancake 2-D cavitons are not described by the $1 \mathrm{D}$ approximation. In general, in the 2-D case the same accelerated distribution as in $1 \mathrm{D}$ can be produced with either smaller pump amplitudes or $T_{e}$.

As the SLT acceleration rate is defined by the wave energy in collapsing cavitons, the acceleration efficiency near the plasma resonance is greater than that in the upper hybrid layer due to $\operatorname{OTSI}_{L o}^{U H}$ (section 3.5.2). It is worth to also note that Samimi et al.'s [2014] numerical simulations at $\omega_{u h r}<2 \omega_{c e}$ and $T_{e} \geq 3 T_{i}$ show that the IAPD instability in the UH layer results in collapsing cavitons and concomitant (parallel $\mathbf{B}_{0}$ ) electron acceleration, resembling the SLT process.

Dimant et al. [1992] considered (transverse) electron acceleration by electron Bernstein waves via cyclotron resonance $\omega_{k}-k_{\|} v_{\|}=s \omega_{c e}$ taking account of elastic collisions that return a fraction of accelerated electrons into a narrow acceleration layer. This mechanism may be

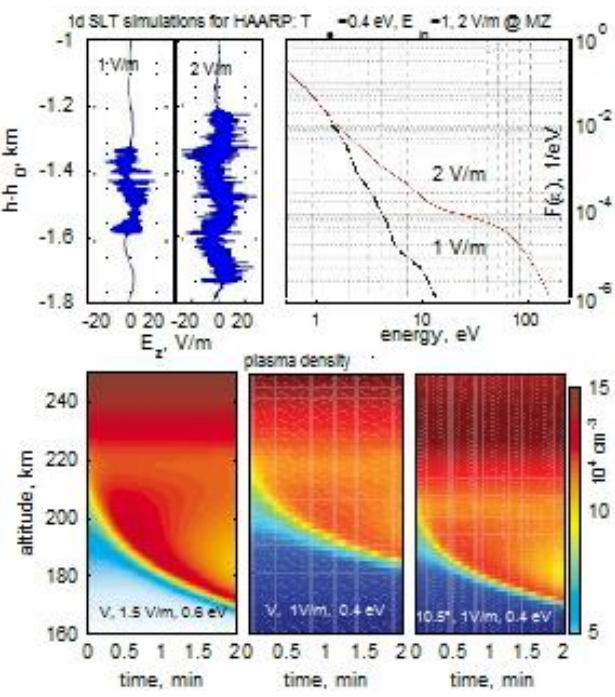

Figure 3.5. Modeling of the accelerated population and descending layers at HAARP. (top) Turbulent electric fields $E_{z}$ and the TDF, $F_{t}(\varepsilon)$, calculated for $\mathrm{MZ}$ injections. (bottom) Time-vs-altitude plots of the artificial plasma density at V and 10.5 S. After Eliasson et al. [2012b; 2015] efficient for a broad wave spectrum near the gyroresonance at altitudes below $200 \mathrm{~km}$ to provide sufficient return flux (albedo). Kuo [2013] included the finite Larmour radius effect, $k_{\perp} v_{\perp} / \omega_{c e}$, enhancing the cyclotron acceleration by short-scale, $\lambda_{\perp}<0.5$ m, UH waves excited via decay PDI ${ }_{U H}^{o}$. This mechanism works below $h_{u h}$ and over the gyroresonance, i.e., $s \omega_{c e}<\omega_{u h r}<\omega_{0}$. Recent Vlasov simulations of electron acceleration by 2 $\mathrm{V} / \mathrm{m}$ O-mode waves in the upper hybrid layer [Najmi et al., 2016; 2017] have shown that the evolution of the transverse electron distribution for $f_{o}$ below and above $4 f_{c e}$ differ drastically. Namely, stochastic bulk heating occurs at $f_{o}<4 f_{c e}$ and otherwise acceleration of suprathermal tail electrons. 


\section{Active Experiments}

\subsection{Stimulated Electromagnetic Emissions (SEEs)}

A HF powerful electromagnetic wave (pump wave, $\mathrm{PW}$ ) of O-mode polarization, radiated by ground located HF transmitters, may generate in the ionospheric disturbed volume (IDV) secondary electromagnetic waves at frequencies ranging from $f_{0}-200 \mathrm{kHz}$ to $f_{0}+600 \mathrm{kHz}$, where $f_{0}$ is a PW frequency. This phenomenon, termed Stimulated Electromagnetic Emissions (SEEs) occurring as a result of various wave-plasma processes, was discovered in ionospheric modification experiments at the EISCAT heating facility [Thidé et al., 1982]. The generation of electromagnetic emissions stimulated by a high-power short-pulse wave in the HF modified ionosphere, later termed diagnostic SEE, was observed in 1981 at the Zimenky heating facility. SEE has become a very useful tool to study nonlinear processes in heating experiments as both the short timescale ponderomotive nonlinearities, leading to Langmuir and upper hybrid turbulence, and the long timescale thermal nonlinearities, leading to the excitation of plasma density irregularities, are involved in the SEE generation [Erukhimov et al., 1987; Stubbe and Hagfors, 1997, Sergeev at al., 1999; Leyser, 2001].

Since the first observations of SEE, a great variety of their spectral components has been identified in the PW sidebands (more than 20 to date). The basic SEE components were summarized by Stubbe et al. [1984], Frolov et al. [2001], and Leyser [2001]. Their spectral features have been characterized by numerous experiments with various ionospheric conditions, PW frequencies and powers, pumping schemes, and preconditioning. Under steady state conditions, there are several basic downshifted SEE components at frequencies below $f_{0}$. These include the downshifted maximum $(D M)$ with the frequency offset $\Delta f^{-} \approx 8-18 \mathrm{kHz}$, which is the most intense SEE feature between gyroharmonics [Stubbe et al., 1984; Leyser et al., 1993, 1994; Leyser, 2001; Sergeev et al., 2006]; the thermal narrow continuum $\left(N C_{\mathrm{th}}\right)$ between the $D M$ and $f_{0}$, generation of which is the result of the TPI development [Leyser, 2001; Sergeev et al., 2006]; the ponderomotive narrow continuum $\left(N C_{\mathrm{p}}\right)$ just below $f_{0}$ by a few, up to $40, \mathrm{kHz}$, which results from the PDI development [Frolov et al., 2004]; and the broad continuum (BC), extending down to $\Delta f^{-} \approx 50$ $150 \mathrm{kHz}$ below the DM [Leyser et al., 1993; Sergeev et al., 2006].

Figure 4.1 presents SEE spectra for $f_{0}=5455$ and $5745 \mathrm{kHz}$, near and slightly above the $4 f_{\mathrm{ce}}$, respectively. The basic upshifted SEE spectral components are the upshifted maximum $(U M)$, which is a narrow peak at $\Delta f^{+} \approx 7-12 \mathrm{kHz}$ [Stubbe et al., 1984; Leyser, 2001; Sergeev et al., 2006]; the broad upshifted maximum $(B U M)$, at $\Delta f^{+} \approx 15-150 \mathrm{kHz}$ at $f_{0}$ close to or slightly above $s f_{\mathrm{ce}}$ for $s \geq 3$ [Stubbe et al., 1994; Frolov et al., 2001; Leyser, 2001; Sergeev et al., 2006], and the broad upshifted structure (BUS), which is a wideband emission at $\Delta f^{+} \approx 15-100 \mathrm{kHz}$ when $f_{0}$ exceeds $s f_{\text {ce }}$ [Sergeev et al., 2006]. 
Significantly, the SEE features strongly depend on $f_{0}$ when it is close to $s f_{\text {ce }}$ [Frolov et al., 2001; Leyser et al., 1993; 1994; Leyser, 2001; Sergeev et al., 2006; Stubbe et al., 1994]. According to Stubbe et al. [1994], the SEE components may be divided into the "gyrofeatures" that exist only for $f_{0} \approx s f_{\text {ce, }}$, and the "universal features", that exist for all pump frequencies, but strongly change their properties at $f_{0} \approx s f_{\text {ce. }}$. The basic universal features are $N C_{\mathrm{p}}, N C_{\mathrm{th}}, B C, D M$, and $U M$, whereas the basic gyrofeatures are the BUM, BUS, and broad symmetrical structure (BSS) [Stubbe and Kopka, 1990]). However, the universal features' characteristics also depend on $f_{0}$ even outside the gyroharmonic frequency range [e.g., Sergeev et al., 2006].

Comprehensive investigations of the steady state SEE features in relation to $f_{0}$ were conducted in $1996-2000$ at the SURA facility in the available frequency range from 4.3 to 9.5 MHz between $3 f_{\text {ce }}$ and $7 f_{\text {ce }}$ [Frolov et al., 2001; Sergeev et al., 2006]. In these experiments, $f_{0}$ was stepped by 20 or $50 \mathrm{kHz}$, for $f_{0}$ far from

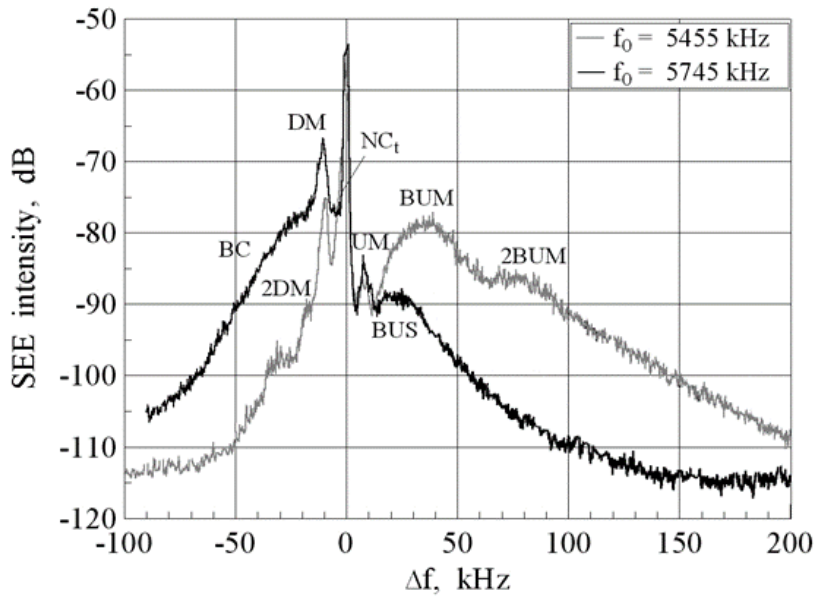
gyroharmonics, and by $1-5 \mathrm{kHz}$ at $f_{0} \approx s f_{\text {ce }}$. Due to the increasing solar activity and thus [Sergeev et al., 2006]. $f_{O} F 2$ in 1996 - 2000, the SEE spectra were measured with the increasing $f_{0}$, according to the rule that the difference between $f_{0}$ and $f_{O} F 2$ is less than $1 \mathrm{MHz}$. The measurements were conducted under quiet ionospheric conditions during day and evening hours using an effective radiated power $P_{0} \approx 30-60 \mathrm{MW}$, corrected for one way linear absorption in the $D$ and $E$ ionospheric layers.

The results of SEE spectral measurements for $f_{0}=4.3-9.5 \mathrm{MHz}$ are summarized in Figure 4.2. One can clearly see such well-known SEE features as:

(i) a linear increase of the $D M$ offset frequency, $\Delta f_{\mathrm{DM}}$, from $\sim 9 \mathrm{kHz}$ to $\sim 18 \mathrm{kHz}$ with increasing $f_{0}$, far from the gyroharmonics, and the decrease of the $\Delta f_{\mathrm{DM}}$ magnitude at $f_{0} \cong s f_{\mathrm{ce}}$ due to the stronger suppression of the $D M$ intensity at its low-frequency flank;

(ii) suppression of the $D M$ and $B C$ near the gyroharmonics, $f_{0} \cong s f_{\mathrm{ce}}$;

(iii) $B U M$ generation when $f_{0}$ is close to, or slightly above $s f_{\mathrm{ce}}$, as well as the growth of the $B U M$ peak frequency with increasing $\delta f=f_{0}-s f_{\mathrm{ce}}$;

(iv) a local enhancement of both the $D M$ and $B C$ at $f_{0}$ slightly below (for $\delta f \approx-(20-40) \mathrm{kHz}$ ) the gyroharmonic for $s=5-7$, and the absence of such enhancement for $s=4$;

(v) contraction of the BUS generation frequency band with increasing $s$ and approach of the frequency subrange of the highest $B U S$ intensity to the gyroharmonic.

In addition to these well-documented SEE features, Figure 4.2 shows that: 
(vi) at the stationary stage of pumping, the basic downshifted components of the thermal origin $\left(N C_{\text {th }}, D M\right.$, and $\left.B C\right)$ are most intense in the frequency range between $4 f_{\text {ce }}$ and $5 f_{\text {ce }}$;

(vii) the frequency band, where the $N C_{\text {th }}$ and $D M$ intensities have a maximum (for $\delta f \approx 600-800$, $100-400,50-200,100-200$ and $50-150 \mathrm{kHz}$ above the $3^{\text {rd }}-7^{\text {th }}$ gyroharmonic, respectively), constricts and approaches a gyroharmonic with increasing $s$ from 3 to 5 , whereas for $s>5$ these characteristics remain almost the same. The existence of the similar frequency dependence for the BUS features was found by Frolov et al. [2000] and can also be seen in Figure 4.2;

(viii) a faster increase of the $D M$ and $B C$ intensity with increasing $f_{0}$ in the frequency range $\delta f \leq$ $400 \mathrm{kHz}$ above the gyroharmonic, as compared with the rate when $f_{0}$ decreases from the gyroharmonic resonance.

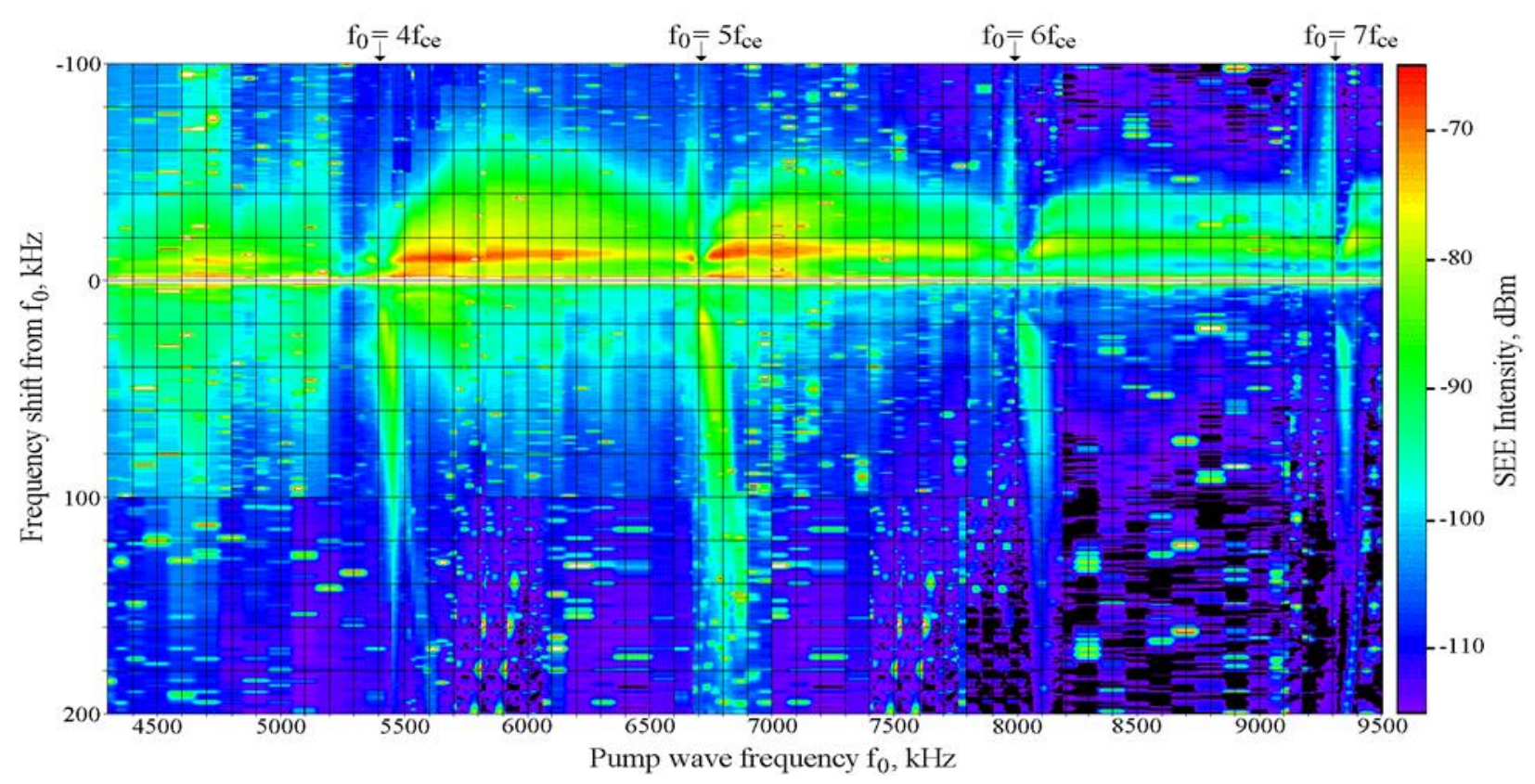

Figure 4.2. Variations of the SEE spectral structure in the frequency range $f_{0}=4.3-9.5 \mathrm{MHz}$ [Frolov et al., 2001].

Based on the data shown in Figure 4.2, it can be concluded that gyro-effects exert the strong impact on the stationary SEE features for all frequencies between neighboring gyroharmonics, and that the impact is stronger for smaller harmonic numbers. It should be noted that this is the natural behavior for resonance type phenomena.

To analyze the dependence of the stationary SEE features on $f_{0}$ in more details, the family of SEE spectra is presented in Figure 4.3 for five characteristic frequency offsets from the gyroharmonic, with $\delta f \approx-100 \mathrm{kHz},-(20-40) \mathrm{kHz}, \sim 0,+(20-40) \mathrm{kHz},+200$ and $+400 \mathrm{kHz}$ (first-sixth column, respectively). These are obtained near the $4^{\text {th }}-7^{\text {th }}$ gyroharmonics (first-fourth row, respectively) [Sergeev et al., 2006]. The spectra at $\delta f \approx 0, f_{0} \cong s f_{\mathrm{ce}}$, (the "Resonance range", 
range I in Figure 4.3) demonstrate strong suppression of all thermal emission components (DM, $B C$, and $\left.N C_{\text {th }}\right)$, whereas the ponderomotive $N C\left(N C_{\mathrm{p}}\right)$ and the first $B U M(B U M-1)$ are generated. In the second frequency subrange, $\delta f \approx-100 \mathrm{kHz}$ (the "Weak emission range", range IV in Figure 4.3), the emissions are rather weak. This is particularly evident for the $B C$, which can be identified here below the $7^{\text {th }}$ gyroharmonic only. In this range, the $D M$ and second $D M$ are the most pronounced structures in the SEE spectra, with their maximum steady state intensity below the $5^{\text {th }}$ gyroharmonic. It should be noted that the weak signal for $s=4$, with a symmetrical spectral form, is transmitter noise.

In the third frequency subrange, $\delta f \approx-(20-40) \mathrm{kHz}$ (the "Below-gyroharmonic range", range $\mathrm{V}$ in Figure 4.3), the $D M$ and $B C$ intensities maximize at $s=5$, decreasing gradually with increasing
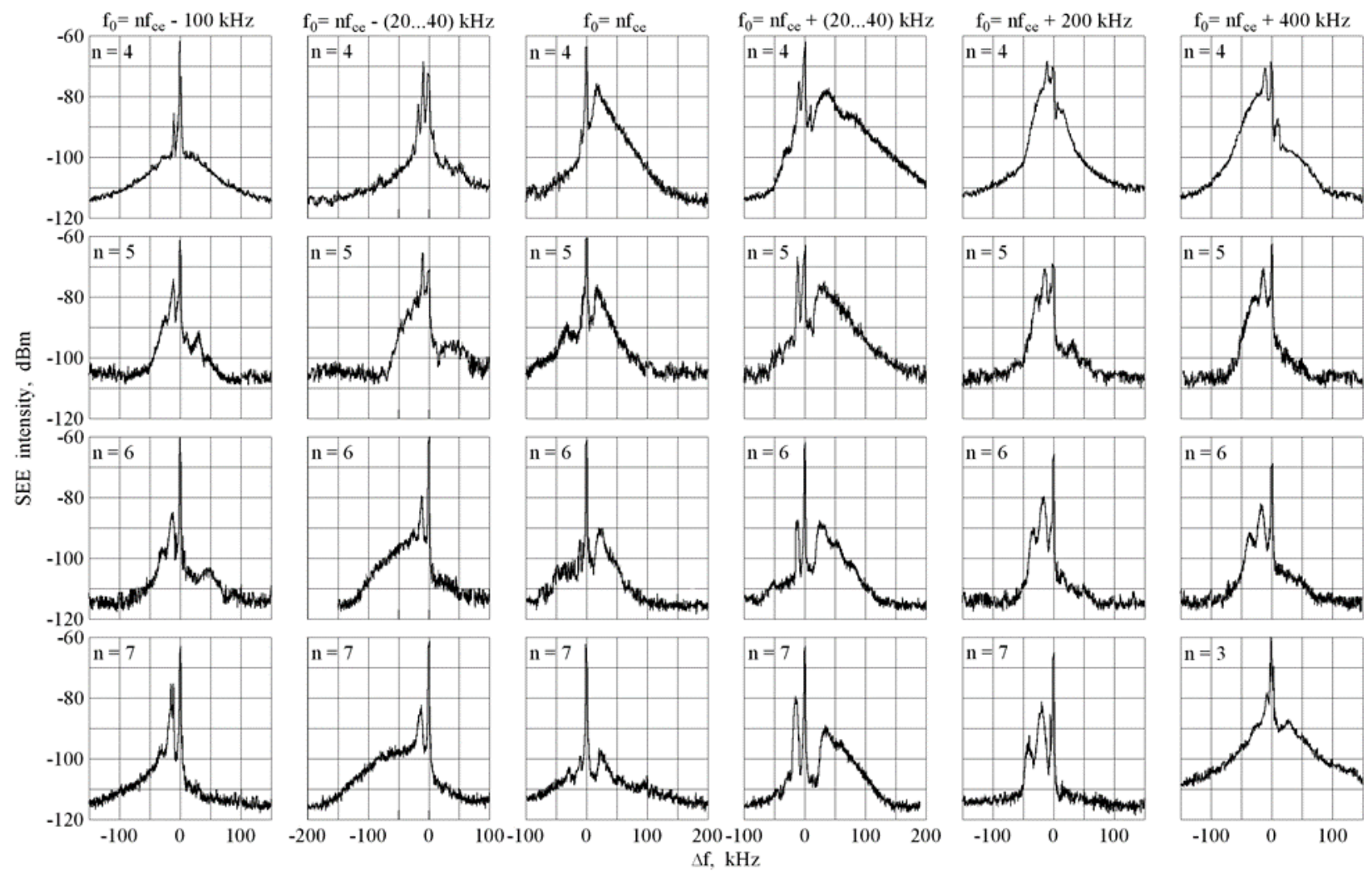

$S$, Figure 4.3. A family of SEE spectra for characteristic frequency offsets of the PW frequency from the electron gyroharmonics $\delta f=f_{0}-n f_{c e}$ (columns): $\delta f \approx-100 \mathrm{kHz}$ (range IV), $\delta f \approx-(20-40) \mathrm{kHz}$ (range V), $\delta f \approx 0,\left(f_{0}-n f_{c e}\right.$ (columns): $\delta f \approx-100 \mathrm{kHz}$ (range IV), $\delta f \approx-(20-40) \mathrm{kHz}$ (range V), $\delta f \approx 0,\left(f_{0} \approx n f_{c e}\right)$ (range I) (range I), $\delta f \approx(20-40) \mathrm{kHz}$ (range II), $\delta f \approx 200 \mathrm{kHz}$ and $\delta f \approx 400 \mathrm{kHz}$ (range III) for $n=4-7$ (rows). At the bottom right panel the SEE spectrum for $f_{0}=4400 \mathrm{kHz}(n=3)$ is additionally plotted. Interferences on spectra are removed manually [Sergeev et al., 2006].

whereas the width of the $B C$ increases with increasing $s$. This observation indicates that the width of the $\mathrm{BC}$ is not determined directly by its intensity. Below the $4^{\text {th }}$ gyroharmonic, the SEE spectrum comprises multiple $D M \mathrm{~s}(D M, 2 D M$, and $3 D M)$, while the $B C$ is either absent or barely noticeable over the noise background. Multiple $D M$ s can be also seen in the SEE spectra for $s=5$ and 6 on the background of the $B C$. In the fourth frequency subrange, $f \approx+(20-40) \mathrm{kHz}$ (the "Above- 
gyroharmonic range", range II in Figure 4.3), where both the strongest and widespread $B U M-2$ spectra occur, the $B U M-2$ is stronger near the $4^{\text {th }}$ and $5^{\text {th }}$ gyroharmonics. Here the $B C$ is not seen and the $D M$ intensity depends strongly on the frequency offset from gyroresonance. In the fifth frequency subrange, $f \approx+(200-400) \mathrm{kHz}$ (the "Strong emission range", range III in Figure 4.3), the enhancement of the $N C_{\mathrm{th}}, D M, B C$, and $B U S$ is observed with the strongest steady state intensity above the $4^{\text {th }}$ gyroharmonic. The $D M$ steady state intensity decreases progressively with increasing $s$. For $s=5-7$, the $B C$ is not distinguished in the steady state spectra that are dominated by the $D M$ and $2 D M$. The $3 D M$ does not appear here, though the difference between the $2 D M$ intensity and the background noise level is more than $15 \mathrm{~dB}$. Between the $3^{\text {rd }}$ and $4^{\text {th }}$ harmonics, the stationary intensity decreases again due to the strong overshoot effect, i.e., strongly decreasing intensities after reaching their maximum in several seconds after PW turn-on [Sergeev et al., 1999].

The $D M$ is the most prominent and commonly observed downshifted SEE spectral feature at the frequency offset $\Delta f^{-} \approx 2 \times 10^{-3} f_{0}$. This relation was initially found in experiments at the EISCAT heating facility [Stubbe et al., 1984] and verified later in experiments at SURA [Leyser et al., 1994]. It should be stressed that, though the $D M$ peak frequency increases with $f_{0}$, the highest $D M$ frequency components at about $\Delta f^{-} \approx 7-8 \mathrm{kHz}$ do not significantly depend on $f_{0}$. Notice that this frequency offset is close to the lower hybrid frequency at SURA. Under evening conditions, the DM generation threshold is about 0.5 - 1 MW ERP, which is close to the threshold of the development of anomalous absorption related to artificial small-scale irregularities with $l_{\perp} \leq 30 \mathrm{~m}$, that have the thermal origin. When $f_{0}$ is near $s f_{\text {ce }}$ and the $B C$ is suppressed in the SEE spectra, sometimes additional $D M$ features (2DM and $3 D M$ ) further downshifted from, and weaker than, the prime $D M$ can be well distinguished (see Figure 4.3). The $2 D M$ and $3 D M$ are observed at approximately multiple frequency shifts (2 and 3) $\Delta f_{\mathrm{DM}}$ with intensities decreasing by 9 to $13 \mathrm{~dB}$. The $D M$ is not detectable in a narrow range near the gyroresonance, $f_{0} \cong s f_{\text {ce }}$, with the width about $2-6 \mathrm{kHz}$ for $s=4$ that decreases to $0.2 \mathrm{kHz}$ for $s=7$ [Leyser et al., 1994; Leyser, 2001]. This behavior allows to determine with high resolution the gyrofrequency in the heating region, as well as to find a gyroresonance immediately during heating experiments. Note that the $D M$ is observed even at $f_{O} F 2$ below $f_{0}$ by $100-200 \mathrm{kHz}$.

The upshifted maximum $(U M)$ appears in the SEE spectrum almost symmetrically to the $D M$, at $\Delta f^{+} \approx \Delta f^{-}(D M)-2 \mathrm{kHz}$ [Sergeev et al., 2006]. It is observed only at ERP $P_{0} \geq 30 \mathrm{MW}$, much greater than the $D M$ threshold. Far from the gyroresonance, the $U M$ intensity is smaller than that of the $D M$ by $10-20 \mathrm{~dB}$, but is comparable or even exceeds in the range $\mathrm{I}$, where the DM is strongly suppressed. When $f_{0}$ passes through $s f_{\text {ce }}$ from below, the minimum $U M$ intensity is observed at $f_{0}=f_{01}$, while the minimum of the $D M$ intensity is reached at $f_{02} \approx f_{01}+2 \Delta f^{-}(D M)$, and the minimum of the total SEE intensity occurs at $f_{00} \approx f_{01}+\Delta f^{-}(D M)$ [Sergeev et al., 2006]. This behavior illustrates that the gyroresonance frequency is shifted from the DM suppression frequency $f_{02}$ by about $-10 \mathrm{kHz}$. Note that the actual UM counterpart, dubbed the intermediate 
downshifted maximum (IDM), was recently revealed during the March 2011 HAARP campaign [Grach et al., 2016].

The broad continuum (Figures 4.2 and 4.3) is observed in a wide frequency range between successive gyroharmonics, in the subranges III and IV [Frolov et al., 2001; Leyser, 2001; Sergeev et al., 2006]. The BC intensity and width (up to $\Delta f^{-} \approx 100 \mathrm{kHz}$ ) maximize in the range III between $4 f_{\mathrm{ce}}$ and $5 f_{\mathrm{ce}}$, and a strong overshoot effect (up to $25 \mathrm{~dB}$ ) occurs between $3 f_{\text {ce }}$ and $4 f_{\text {ce }}$ [Sergeev et $a l ., 1999]$. The $B C$ intensity significantly decreased for $f_{0}>5 f_{\text {ce. }}$ It should be mentioned that for $s$ $\geq 5$ the $B C$ strong intensification and broadening (up to $\sim 150 \mathrm{kHz}$ ) are observed in a narrow frequency range $\delta f \approx 20-40 \mathrm{kHz}$ below the gyroharmonics (range $\mathrm{V}$ ). In the weak emission range $\left(\delta f \approx-(100-300) \mathrm{kHz}\right.$, range IV), as well as for pumping near the critical frequency $f_{O} F 2$, the $B C$ generation is suppressed and the SEE spectrum is dominated by the $D M$ family. The $B C$ development follows the formation of small-scale irregularities and shows strong dependence on pre-conditioning. Under evening conditions, the $B C$ generation threshold is about $0.5-1 \mathrm{MW}$ ERP.

The narrow continuum $(N C)$ in the SEE spectrum is observed at frequencies $f_{0}$ and is distinguished by the fast, $2-3 \mathrm{~dB} / \mathrm{kHz}$, decrease of the spectral intensity when $\Delta f^{-}$is increased. A remark is in order. Two different SEE components have been identified at low ERPs. First, the ponderomotive $N C\left(N C_{\mathrm{p}}\right)$, which results from the PPI development and is detected both at the initial stage $(100-200 \mathrm{~ms}$ after $\mathrm{PW}$ switch-on $)$ and when $f_{0} \cong s f_{\mathrm{ce}}$ and the thermal parametric instability does not develop. Second, the thermal $N C\left(N C_{\mathrm{th}}\right)$, which is observed in the frequency range between $f_{0}$ and the $D M$ at the thermal stage of $\mathrm{PW}$-plasma interactions and results from the TPI development. The temporal and spectral characteristics of the $N C_{\mathrm{p}}$ have been explored in detail by Frolov et al. [2004]. It has been shown that just at the beginning of pumping the $N C_{\mathrm{p}}$ is the only SEE feature occupying the range $\Delta f^{-} \approx 0-30 \mathrm{kHz}$. Then, after the development of the UH turbulence, the $N C_{\mathrm{p}}$ intensity is strongly decreased due to suppression of the parametric decay instability by the anomalous absorption due to the growth of the small-scale irregularities. At this stage, the $N C_{\text {th }}$ appears in the SEE spectra and becomes the dominant feature in the frequency range between $f_{0}$ and the $D M$. Temporal and spectral characteristics of the $N C_{\text {th }}$ as well as its gyro features have been considered in detail by Sergeev et al. [2006].

The features of the broad upshifted maximum $(B U M)$, occurring when $f_{0}$ is near but greater $s f_{\text {ce }}$ were studied by [Leyser et al., 1990; 1993; 2001; Stubbe et al., 1994]. The BUM is a composition of two different components. The first, $B U M-1$, dominates the $B U M$ spectrum for $\left|f_{0}-s f_{\text {ce }}\right| \leq 10$ $\mathrm{kHz}$ and shows faster development than the second one, is generated in the immediate vicinity of the gyroharmonic. It shows a weak dependence of the frequency offset of the peak intensity, $\Delta f_{\text {BUM }}$ $=f_{\text {peak }}(B U M-1)-f_{0}$, on $f_{0}$. The $B U M$ intensity maximizes at $\delta f \approx 0$ where the $D M$ generation is hampered [Leyser et al., 1990; 1993; 2001], as well as at vertical injections. There are reasons to assume that the $B U M-1$ is generated by the parametric decay instability. 
The BUM-2 component [Leyser, 2001] is generated when $\delta f>0$ and dominates the BUM spectrum for $\delta f \geq 20 \mathrm{kHz}$. Its peak frequency shows a stronger dependence on $f_{0}$ than that of $B U M$ 1. For $\delta f \geq 40 \mathrm{kHz}, \Delta f_{\mathrm{BUM}-2}$ can be approximated as $\Delta f_{\mathrm{BUM}-2}=\delta f$. The $B U M-2$ intensity maximizes at $\delta f=30-40 \mathrm{kHz}$, where the occurrence of multiple maxima (up to three) is observed in the spectrum. Under evening conditions, the threshold for the $B U M-2$ generation is about $3-5 \mathrm{MW}$ ERP. The key $B U M-2$ features are explained by a four-wave process near the upper hybrid resonance altitude when the pump frequency is close to, but larger than the gyroharmonic [Huang and Kuо, 1994].

The broad upshifted structure $(B U S)$ is observed in the strong emission range III when the thermal SEE features, the $D M$ and $B C$, maximize. Under evening conditions, the $B U S$ generation threshold is about 3-6 MW ERP. The widest range of $f_{0}, 4300 \mathrm{kHz} \leq f_{0} \leq 3.5 f_{\mathrm{ce}}$, is observed for the $B U S$ between $3 f_{\text {ce }}$ and $4 f_{\text {ce }}$ (see Figures 4.2 and 4.3 ), where $4300 \mathrm{kHz}$ is the lowest frequency available at SURA. The narrowest one, of only a few tens kHz near $f_{0} \approx 5 f_{\mathrm{ce}}+200 \mathrm{kHz}$, is between $5 f_{\mathrm{ce}}$ and $6 f_{\mathrm{ce}}$. For $s>6$ the $B U S$ can hardly be distinguished above the noise. As a rule, the $B U S$ has a weak spectral maximum (see Figure 4.2). Its frequency shift slowly decreases with $f_{0}$ from $\Delta f_{\text {BUS peak }} \approx 15-30 \mathrm{kHz}$ between the $3^{\text {rd }}$ and $4^{\text {th }}$ gyroharmonic to $\approx 14-22 \mathrm{kHz}$ between the $4^{\text {th }}$ and $5^{\text {th }}$. Note that despite a number of their common properties, the $B U S$ and $B U M$ depend on $f_{0}$ differently.

Another SEE upshifted structure is the broad symmetrical structure (BSS), which consists of two broadband maxima around $f_{0}$ at $\left|\Delta f^{ \pm}\right| \approx 15-30 \mathrm{kHz}$. This structure was observed only for $f_{0} \approx$ $3 f_{\mathrm{ce}}$ [Stubbe and Kopka, 1990; Stubbe et al., 1994]. Also, an upshifted wideband emission (UWE) has been observed for $f_{0}=4785 \mathrm{kHz}$ at $\Delta f^{+} \approx 50-400 \mathrm{kHz}$ with the spectral intensity maximum in the range $\Delta f^{+} \approx 100-200 \mathrm{kHz}$ [Leyser, 2001]. Its peak intensity is reached in $1 \mathrm{~s}$ after heating is switched on, along with the excitation of small-scale irregularities near the reflection altitude.

The above data demonstrate the strong dependence of the SEE features on the gyroresonance, which is observed over a broad frequency range, intensifying at smaller $s$. Besides, it has been found that the steady state intensity of the thermal emission components $\left(N C_{\mathrm{th}}, D M\right.$, and $\left.B C\right)$, as well as the $B U S$, maximizes above the $4^{\text {th }}$ gyroharmonic. The stronger decrease of the $D M$ stationary intensity relative to that of the $N C_{\text {th }}$ for $f_{0}<4.7 \mathrm{MHz}$ explains why the $D M$ cannot be distinguished from the continuum background in the EISCAT experiments at $f_{0}<4.3 \mathrm{MHz}$ [Leyser et al., 1990].

Physical models for the basic SEE spectral components have been extensively discussed elsewhere [e.g., Leyser, 2001]. Particularly, the downshifted features, except for the $N C_{\mathrm{p}}$, are generated via conversion of UH waves on small-scale, magnetic field-aligned irregularities into electromagnetic emissions. The formation of the UH spectrum (as explained in section 3) occurs due to different nonlinear processes in the heated volume, like a three-wave (decay) interaction between the PW, upper hybrid and lower hybrid waves (for the $D M$ family and $U M$ ), induced 
scattering of UH waves off thermal ions (for the $B C$ ), four-wave parametric instability (for the $B U M$ ), etc. [e.g., Leyser, 2001 and references therein]. The suppression of the UH-related SEE features near the gyroharmonics is attributed to the proximity of the PW frequency to the double resonance frequency $f_{0}=s f_{\mathrm{ce}}=f_{\mathrm{UH}}[$ Mjølhus, 1993; Grach et al., 1994].

The HAARP heating facility, which has at present the highest possible effective radiated power (up to $4 \mathrm{GW}$ ) and can operate at the lowest PW frequency (down to $2.7 \mathrm{MHz}$ ), recently revealed some new SEE components, namely: 1) the emission peaks at frequency offsets from the PW frequency of approximately $\pm 30 \mathrm{~Hz}$, that are generated due to stimulated Brillouin scatter instability and provide a new diagnostic tool for determining the state of the HF-modified ionosphere [Bernhardt et al., 2010]; 2) downshifted and upshifted narrow peaks with frequency offsets from $f_{0} \approx 2 f_{\text {ce }}$ of several tens of $\mathrm{Hz}$ located near harmonics of ion cyclotron frequency, generated by parametric decay of HF-induced electron Bernstein waves to multiple electron and ion Bernstein waves [Bernhardt et al., 2011]; 3) a broad downshifted emission spectral feature, which is observed near the third and fourth electron gyroharmonic in a wide frequency range $\Delta f^{-}$ $\approx(40-220) \mathrm{kHz}$ [Sergeev et al., 2016]. These observations demonstrate the wealth of HF-induced plasma processes in the ionospheric plasma.

We have considered only stationary SEE features. Measurements performed at SURA [Sergeev et al., 1997; 1999] have shown that the temporal evolution and decay of various SEE components after PW switch-on/off also depends on many parameters such as the PW frequency and power, time of day, ionospheric conditions, the duty cycle of pumping, etc. A detailed analysis of the overall observations is beyond the scope of the present review.

Different methods to study features of the low-frequency artificial ionospheric turbulence has been developed based on SEE [Erukhimov et al., 1988; Kagan and Frolov et al., 1996]. They were successfully employed to study diurnal variations of the features of small-scale irregularities, peculiarities of transport processes in the upper ionosphere, and artificial plasma density perturbations.

\subsection{Artificial Field-Aligned Irregularities (FAIs)}

One of the most important effects produced by the interaction of a high power HF wave with the $\mathrm{F}$ region ionospheric plasma is the generation of artificial magnetic field-aligned irregularities (FAIs) and ducts. In this review, the HF-induced irregularities are divided into three classes, such as small-scale (SSIs), medium-scale (MSIs), and large-scale (LSIs), taking into account the mechanisms responsible for their generation. The practical importance of FAIs is their ability to form an effective target for radar backscattering as well as causing anomalous absorption of the pump wave. Near the pump wave reflection height, this anomalous absorption may dominate over collision-based absorption [Robinson, 1989]. Backscatter from heater-induced FAIs was first observed by Thome and Blood [1974] using the Platteville ionospheric heater in Colorado, USA. 
These early observations established that the scattering is highly aspect-sensitive and observed only when the Bragg-Woolf condition is fulfilled for incident and scattered waves.

Substantial changes to the electron density have been observed on a diverse hierarchy of spatial scales, from large-scale density depletions in the heated region to small-scale irregularities aligned with the geomagnetic field as detected by rockets and VHF-UHF backscattering [e.g. Kelley et al., 1995].

\section{Small-Scale Irregularities (SSIs)}

The excitation of SSIs with the transverse scale size $l_{\perp} \approx 1-100 \mathrm{~m}$ occurs via the Thermal Parametric Instability (section 3.6.3) and leads to efficient dissipation of the EM pump wave near the upper-hybrid resonance layer (anomalous absorption). The timescale required for the TPI to develop is $1-10 \mathrm{~s}$. This instability is facilitated by pre-existing density irregularities in the resonance region that either could be naturally occurring or remaining after a prior heating. It should be noted that Artificial Super Small-Scale irregularities (ASSI) with $l_{\perp} \approx 0.1-0.2 \mathrm{~m}$, are also observed, but only when the pump frequency is slightly above the gyroharmonic in the heating region.

\section{Medium-Scale Irregularities (MSIs)}

Another thermal instability associated with the formation of field-aligned density structures is the Thermal Self-Focusing Instability. Section 3.6.2 describes TSFI near the plasma resonance altitude. Gurevich et al. [1998] considered TSFI near the upper hybrid resonance altitude as follows. Small-scale density irregularities generated around the upper-hybrid resonance region reduce the local electron plasma density thus producing a refractive, lensing effect on the incident EM wave, which focuses the pump wave and leads to enhancement of the E-field amplitude in this region. Self-focusing of this nature results in further nonlinear enhancement of the electron temperature and causes the evolution of a hierarchy of density-depleted structures, from the smalltransverse-scale irregularities associated with the thermal resonance instability to self-organized density depleted structures of the order of $l_{\perp} \approx(0.2-1.0) \times 10^{3} \mathrm{~m}$ in transverse scale. This increased growth of density-depleted structures further reduces the net electron density in the interaction region and enhances the focusing effect, providing a feedback loop that powers nonlinear growth of the electron temperature around the upper-hybrid region. The timescale for this process to manifest is of order $\sim 1$ min, making the self-focusing instability relatively slow compared to the parametric decay instability or thermal resonance processes.

\section{Large-Scale Irregularities (LSIs)}

The term "large-scale" in this context corresponds to structures of a $l_{\perp} \approx 1-10 \mathrm{~km}$ scale-size perpendicular to the geomagnetic field. Generation of these irregularities inside the HF radio beam 
is determined by plasma heating. Contributing mechanisms behind this enhanced temperature could include collisional dissipation of the high-power pump and "anomalous" absorption of excited plasma waves. The ponderomotive force is enhanced close to the pump reflection height due to the high-amplitude standing wave, and can also contribute to large-scale, nonlinear modification of the plasma in the heated volume.

There are plasma irregularities occurring due to the plasma density variations on the scale of the high power radio wave beam with sizes from several tens to hundreds of kilometers. This type of disturbances includes the formation of a defocusing lens in the daytime ionosphere at altitudes of 130 to $180 \mathrm{~km}$ due to variations in the ionization-recombination balance in the plasma heated by a high-power radio wave, as well as the formation of a focusing lens at altitudes of 200 to 400 $\mathrm{km}$ due to the thermal diffusion redistribution of the heated plasma in the ionospheric $F_{2}$ region [Gurevich, 1978]. It has been found that: 1) a defocusing lens is formed in the daytime ionosphere when rather high plasma density is observed in the $E$ and $F_{1}$ ionospheric layers; 2) its formation is observed for both $\mathrm{O}$ - and $\mathrm{X}$-mode waves; 3 ) the focal length of such a lens is of the order of 50 $70 \mathrm{~km}$; 4) typical times of its growth and relaxation are about $20 \mathrm{~s}$; 5) such lens causes the decrease of the pump wave flux energy in the $F_{2}$ region by $10-20 \mathrm{~dB}$.

The joint action of a defocusing lens and higher absorption of powerful HF waves in the lowerionosphere $D$ and $E$ layers leads to the effect that under daytime conditions the large decrease of PW intensity by $20-30 \mathrm{~dB}$ takes place at $F_{2}$ region heights. Besides, the presence of photoelectrons in the daytime ionosphere hinders parametric instabilities by enhancing the Landau damping of plasma waves. This and the larger absorption of the pump wave in the daytime loweraltitude $F_{2}$-peak impedes the interaction between high-power radio waves and the F-region ionosphere.

On the contrary, under evening and night conditions, the aforementioned effects are greatly reduced, and the pump energy is easily penetrated into the $F_{2}$ region, leading to the enhanced electron temperature and depleted plasma density. As this takes place between $200-400 \mathrm{~km}$, a focusing lens is formed, which can strongly affect the propagation of incident radiowaves.

\section{Super Large-Scale Irregularities and Atmospheric Gravity Waves (AGWs)}

The satellite radio tomography technique has been applied during heating experiments at SURA since August 2002 to explore large-scale spatial structures in the HF-perturbed ionosphere [Tereshchenko et al., 2004; Frolov et al., 2007; Kunitsyn et al., 2010, 2012; Andreeva et al., 2016]. This technique allows exploring the perturbed region with a 10-20 km spatial resolution between $200-700 \mathrm{~km}$ in the satellite orbit plane, usually close to the meridian at mid latitudes. Figure 4.4 [Frolov et al., 2007] shows two tomograms during the evening and night experiments for continuous vertical injections of ordinary waves at $120 \mathrm{MW}$ ERP and frequencies $4.785 \mathrm{MHz}$ close to $f o F 2$ (i.e., the critical density near $3 \times 10^{11} \mathrm{~m}^{-3}$ ). The heater was turned on for $20 \mathrm{~min}$ at about 15 
min before the satellite pass over the heated spot. Dashed and solid lines indicate the radiation pattern $(\sim 60 \mathrm{~km}$ at altitudes $\sim 300 \mathrm{~km})$ of the main antenna lobe and the direction of the geomagnetic field for the SURA facility, respectively.

The tomograms show the difference reconstruction, which reveals a few percent plasma density deviations from the background values at distances about $300 \mathrm{~km}$ around SURA. One can see that the plasma density perturbations in the region, significantly exceeding the radiation pattern, are mainly magnetic field-aligned, and fill the whole altitude range of $200-700 \mathrm{~km}$ for the given experiment geometry. A $20 \%$ cavity in the southern sector of the antenna pattern is surrounded by structures with enhanced density. The night experiment produced stronger, smallerscale perturbations in the larger area than during the evening experiment.

Since 2007, radio tomography experiments at the SURA facility focus on the excitation of wavelike disturbances in the ionosphere, such as traveling ionospheric disturbances (TID) associated with atmospheric gravity waves (AGWs). For these experiments, the heater is turned on about $2-3$ hours before a satellite pass over SURA with 20 or 30 min square amplitude modulation [Kunitsyn et al., 2012]. Figure 4.5 [Andreeva et al., 2016] shows a tomographic
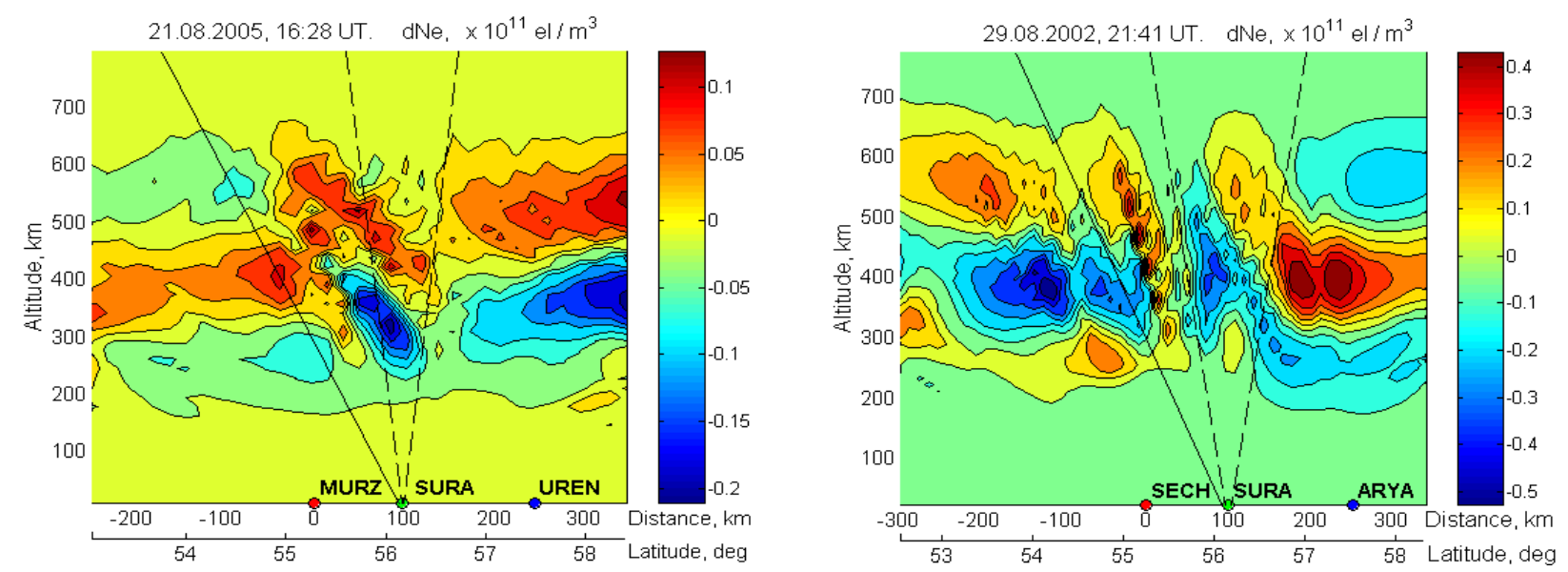

Figure 4.4. Tomographic reconstructions of the ionospheric electron density profile during (left) evening of 21 August 2005 and (right) night of 29 August 2002 [Frolov et al., 2007].

reconstruction of the electron density along the pass of the Cosmos 2407 satellite obtained in the latitude range $35^{\circ}-75^{\circ} \mathrm{E}$ on 18 August 2011. An ordinary wave was radiated into the magnetic zenith $(\mathrm{MZ})$ at $4.875 \mathrm{MHz}\left(f_{o F} \approx 5.3 \mathrm{MHz}\right)$ with $50 \mathrm{MW}$ ERP and a $10 \mathrm{~min}$ on/off duty cycle.

A $\sim 60 \mathrm{~km}$ wide region depleted by $20 \%-30 \%$ in the altitude range of $300-400 \mathrm{~km}$ at MZ is evident. The size of this region corresponds to the main antenna lobe where the strongest HFexcited ionospheric plasma turbulence near the reflection point is anticipated. An enhanceddensity duct in the topside ionosphere above $500 \mathrm{~km}$ is clearly seen. Also, $200 \mathrm{~km}$-wavelength disturbances are observed moving north at the distance up to $1000 \mathrm{~km}$ from SURA. Their speed increases with altitude. Their amplitude is significantly less to the south of SURA and they are barely distinguished from natural electron density variations at $\sim 600 \mathrm{~km}$ south from the heater. 
This is, likely, due to the neutral wind effect. These results can be explained by propagation of an AGW-related TID with $\sim 200 \mathrm{~km}$ wavelengths generated by the periodic heating with the modulation frequencies below the Brunt-Väisälä cutoff frequency [e.g., Chernogor and Frolov, 2013].

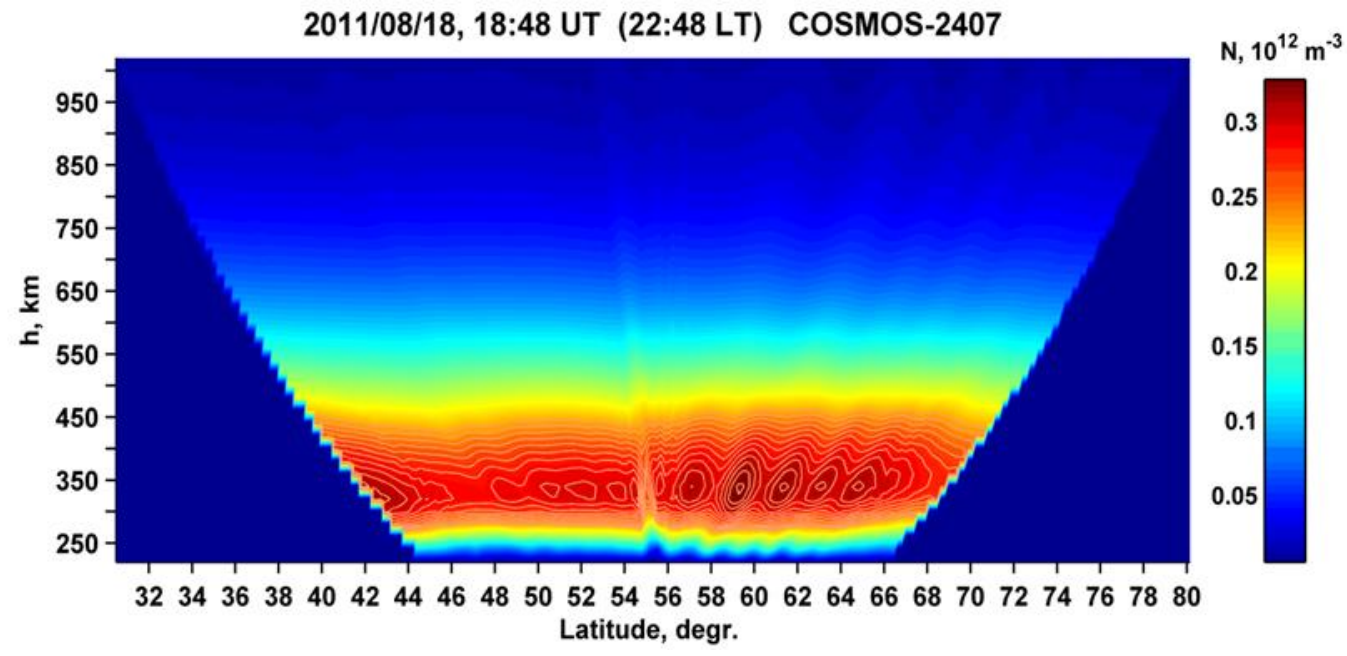

Figure 4.5. A tomographic reconstruction of the ionospheric electron density profile along the Cosmos 2407 satellite path above the SURA facility on 18 August 2011 [Andreeva et al., 2016].

HF-induced AGW/TIDs have also been detected during the dedicated experiments at HARRP [Mishin et al., 2012; Pradipta et al., 2015]. Mishin et al. [2012] explored the thermosphere's response using the CHAllenging Minisatellite Payload (CHAMP) and twin, $25 \mathrm{sec}$ apart, Gravity Recovery And Climate Experiment (GRACE) satellites to measure thermospheric mass densities with 0.1 and $0.2 \mathrm{~Hz}$ sampling rate at 330 and $470 \mathrm{~km}$ in October 2008 and August 2011, respectively. During the four experiments, O-mode waves were transmitted into $\mathrm{MZ}$ at full power 450-650 MW ERP, with either $0.5 \mathrm{~Hz}$ or $5 \mathrm{~Hz} 50 \%$ square modulation. The F2-peak plasma frequency exceeded the heating frequency by $\geq 0.5 \mathrm{MHz}$, ensuring HF beam-ionosphere interaction near 220-240 km. The heater was turned on for $20 \mathrm{~min}$ at about 10-12 min before the overflight of the magnetic zenith. The three experiments were done during exceptionally quiet conditions prior to and during the overflights. Comparing the difference between the quiet-time overflights with and without heating allowed to reveal HF-induced neutral density perturbations of the order of 0.02-0.04 percent. Their spectra were dominated by, respectively, 350 and $900 \mathrm{~km}$ wavelengths at altitudes 330 and $470 \mathrm{~km}$; in agreement with the Brunt-Väisälä cutoff condition.

\subsubsection{Amplitude-Time History of the Pump Wave Reflected from the Ionosphere}

When the pump power exceeds the PPI $I_{L}$ threshold (section 3.3.2), an abrupt decrease of the pump amplitude by $6-20 \mathrm{~dB}$ is observed in the first few milliseconds after PW is turned-on [Erukhimov et al., 1983; Frolov et al., 1997; Sergeev et al., 2004]. This phenomenon is known as 
the striction self-action (SSA). As a result of $\mathrm{PPI}_{\mathrm{L}}$, growth of meter-scale SSI is observed due to the self-focusing instability of HF-induced plasma waves [Perkins, 1974].

During the SSA development at the early stage of pumping, rapid quasi-periodic oscillations (QPO) developed with the growth time 0.1-1 s, while the average amplitude of the received signal increases [Erukhimov et al., 1983; Berezin et al., 1987]. The QPO threshold exceeds that of PPI by approximately $1.5-2$ times. So far, no adequate theoretical explanation of the QPO phenomenon is suggested.

During the next stage of pumping, which is roughly $0.5-10 \mathrm{~s}$ after pump turn-on, SSIs with transverse scales $l_{\perp} \approx 1-100 \mathrm{~m}$ are generated near the upper-hybrid resonance (i.e., $1-10 \mathrm{~km}$ below the pump reflection height) due to the TPI development (section 3.6.3), which is the basis of theory of anomalous absorption due to multiple scattering on SSIs [e.g., Gurevich, 2007]. It was

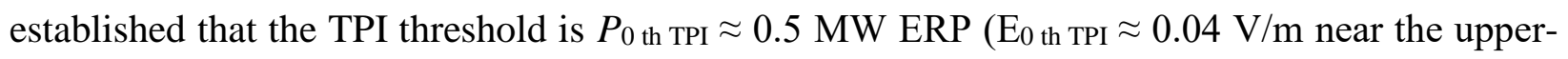
hybrid resonance) in the pump frequency range from 4 to $6 \mathrm{MHz}$ [e.g., Frolov et al., 1997].

For PW powers exceeding $5 \mathrm{MW}$ the anomalous absorption develops much faster, with a typical growth time of about $300-500 \mathrm{~ms}$. It was established that the fast development is due to $\approx 3$-m irregularities that dominate the low-frequency spectrum during the initial stage of TPI [Frolov et al., 1997; 2004]. Frolov et al. [1997] performed probing the HF-modified ionosphere by low-power O-mode diagnostic waves at frequencies upshifted from $f_{0}$ by 130, 150, and 170 $\mathrm{kHz}$, so that their upper-hybrid resonance heights are close to the PW reflection height. These waves damp initially, with a typical decay time of about $200 \mathrm{~ms}$, but then the amplitudes start to restore rapidly coincident with the development of anomalous absorption of both the PW and diagnostic waves. This suggests that $\leq 3$ - $\mathrm{m} \mathrm{SSIs}$ are generated near the PW reflection just after the pump turn-on. It seems plausible that such meter-scale irregularities are the result of the PPI development. These irregularities, extending some kilometers downwards to the pump upperhybrid resonance level, can then initiate a more rapid development of SSIs with scale lengths $l_{\perp} \leq$ $3 \mathrm{~m}$ during the TPI growth.

These results suggest that the $\mathrm{PPI}_{\mathrm{L}}$ development is essential for the subsequent interaction between powerful O-mode waves and ionosphere due to SSIs generation. On the other hand, the SSIs produced by the TPI, screen the reflection level of the PW because of the anomalous absorption [Erukhimov et al, 1983]. So, a mutual influence of the PPI $\mathrm{L}_{\mathrm{L}}$ and TPI creates the complexity of the HF-induced phenomena. Note that the effect of enhanced ionization at high powers adds significantly to this complexity (section 4.6).

During the final stage of a low-power plasma modification, about $10 \mathrm{~s}$ after pump turn-on, strong and fast temporal variations of reflected HF waves appear due to scattering from MSIs with scales $l_{\perp} \geq 100 \mathrm{~m}$. The average signal strength can even become higher compared to the previous anomalous absorption stage. It is believed that the source of MSIs is the TSFI (section 3.6.2) at ERPs exceeding 3 - 5 MW [Gurevich, 1978]. After a few minutes, even larger-scale electron 
temperature and density perturbations develop that scatter and focus the PW energy, thereby modifying the conditions for the wave-plasma interaction and affecting subsequent pump cycles. These irregularities are the reason for the spread- $F$ features on ionograms. To realize the "cold start" conditions during experiments, a few minutes off-period is needed in order to provide either complete decay of LSIs or their transport from the heated volume by the ionospheric wind.

\subsubsection{Temporal Development of FAIs}

The temporal evolution of field-aligned irregularities is determined by the certain key parameters, such as the PW power, frequency, and duty cycle, the presence of both natural and HF-induced irregularities, the PW reflection height and the density gradient in the reflection region, the location of irregularities inside the ionosphere disturbed volume (IDV), and diurnal variations of ionospheric parameters.

At $P_{\text {th TPI }} \leq P_{0}$ eff $\leq 5 \mathrm{MW}$, the scattered wave intensity gradually increases of up to saturation, with a typical growth time $\tau_{\mathrm{gr}} \approx 3-30 \mathrm{~s}\left(\tau_{\mathrm{gr}} \propto P_{0 \text { eff }}{ }^{-1}\right)$. However, as soon as the PW power exceeds $\approx 5 \mathrm{MW}$ ERP, the intensity rapidly increases soon after the pump turn-on. For example, in the experiment where the pump was cycled $10 \mathrm{~s}$ - on and $40 \mathrm{~s}$ - off, the scattered signal amplitude increased exponentially as $e^{\gamma t}$ after a delay time $t_{0} \approx 0.3 \mathrm{~s}$. For $3 \mathrm{~m}$ irregularities and $P_{0 \text { eff }} \approx 100$ MW ERP, the measured value of $\gamma$ is about $1-5 \mathrm{~s}^{-1}$ (or $\tau_{\mathrm{gr}} \approx 0.2-1 \mathrm{~s}$ ). Experimentally, the value of $\gamma$ decreased with increasing irregularity transverse scale length $l_{\perp}$ as $\gamma \propto l_{\perp}^{-2}$.

Experiments also show that at $P_{0 \text { eff }} \geq 10$ MW ERP the maximum is more pronounced for SSIs with scales $l_{\perp} \approx 1.8 \mathrm{~m}$, as compared to that for $l_{\perp} \approx 3 \mathrm{~m}$ [Frolov et al., 1997; Frolov, 2003]. Note also that this maximum has never been observed for $\geq 7-\mathrm{m}$ SSIs that gradually grow until the end. It has been established that $\leq 3 \mathrm{~m}$ SSIs decay concurrently with the development of decameter irregularities [Frolov et al., 1997; 2003].

Results of the Frolov et al. [1997] experiments demonstrate that the delay, $t_{0}$, of the SSIs onset is determined by the growth time of the small-scale turbulence. Significantly, the delay is observed, even though HF-induced irregularities do not decay completely during the turn-off periods. It has been found that $t_{0} \propto l_{\perp}$ and $t_{0} \propto P_{0}$ eff $^{-1}$, having the average magnitude of $t_{0} \approx 50-100 \mathrm{~ms}$ for $l_{\perp} \approx$ $3 \mathrm{~m}$ and $P_{0 \text { eff }} \approx 100 \mathrm{MW}$ ERP. It is also important that SSIs last relatively long after a short-pulse pumping. The lifetime increases with the increase of the scale length $l_{\perp}$. It was also shown that the initially fast stage of anomalous absorption is determined by 3-m SSIs, while decameter SSIs control the subsequent slow stage, and that $3 \mathrm{~m}$ irregularities dominate the SEE generation, at least in the low-sideband frequency range. The latter indicates that SEE can be used as a diagnostic method to study features of both artificially induced and natural small-scale irregularities [Frolov et al., 2004].

The SSI development can also be specified by the time $t_{1}$ during which the intensity of the irregularities reaches either a maximum (for $\leq 5 \mathrm{~m}$ irregularities at $P_{0} \geq 5 \mathrm{MW}$ ERP) or a steady 
state (for $l_{\perp}>5 \mathrm{~m}$ and independent of the scale length for $P_{0 \text { eff }} \leq 5 \mathrm{MW} \mathrm{ERP)}$ ). As a rule, this dependence can be represented in a power-law form: $t_{1} \propto l_{\perp} \beta$ with power index $\beta=0.3-1(<\beta>\approx$ $0.5)$. The dependence of $t_{1}$ on the pump power also has a power-law form: $t_{1} \propto P_{0} \delta$, where $\delta \approx 0.5$ - 1 [Belikovich et al., 1988; Frolov et al., 1997].

\subsubsection{Relaxation of FAIs}

Measuring backscatter from $\approx 1.6-100 \mathrm{~m}$ SSI characterizes their relaxation at $P_{0} \geq 10-20$ MW ERP, when the artificial turbulence has been already saturated [Belikovich et al., 1988]. It was shown that in this case the dependence of the decay time, $\tau_{\mathrm{d}}$ on $l_{\perp}$ can be presented as: $\tau_{\mathrm{d}} \propto$ $l_{\perp}{ }^{\alpha}$, where $\alpha=2$, if $l_{\perp}<l_{\perp}{ }^{*}$ and $\alpha=0.5$, if $l_{\perp}>l_{\perp}{ }^{*}$ [Erukhimov et al., 1987; Frolov et al., 1997]. Here the critical scale length is $l_{\perp}{ }^{*} \approx 7-10 \mathrm{~m}$ and $\tau_{\mathrm{d}} \approx 5-10 \mathrm{~s}$ under evening conditions. During twilight, $\tau_{\mathrm{d}}$ increases by a factor of $1.5-2$. At $l_{\perp}<l_{\perp}{ }^{*}$, the effective diffusion coefficient can be defined as $\mathrm{D}=l_{\perp}{ }^{2} / 4 \pi^{2} \tau_{\mathrm{d}} \approx 1.7 \times 10^{3} \mathrm{~cm}^{2} / \mathrm{s}$, which is close to the coefficient of the ambipolar (electron) diffusion across geomagnetic field lines, $\mathrm{D}_{\mathrm{a} \perp}=\left(T_{\mathrm{e}}+T_{\mathrm{i}}\right) v_{\mathrm{e}} / m \omega_{\mathrm{ce}}{ }^{2} \approx 2 \times 10^{3} \mathrm{~cm}^{2} / \mathrm{s}$. Decay of irregularities with $l_{\perp}>l_{\perp}{ }^{*}$ is determined by the longitudinal ambipolar (ion) diffusion with the coefficient $\mathrm{D}_{\mathrm{a} \|} \equiv l_{\|}{ }^{2} / 4 \tau_{\mathrm{d}}=\left(T_{\mathrm{e}}+T_{\mathrm{i}}\right) / M_{\mathrm{i}} v_{\mathrm{in}} \approx(1-2) \times 10^{10} \mathrm{~cm}^{2} / \mathrm{s}$. These experiments have also revealed the unipolar electron and ion diffusion regimes at $l_{\perp}\left\langle l_{\perp}{ }^{* *}\right.$ and $l_{\perp}>l_{\perp}{ }^{* *}$, respectively, where $l_{\perp}{ }^{* *} \sim$ $10 \mathrm{~m}$.

Under evening and night conditions, scattered signals usually exhibit a clearly defined twostage decay pattern [Belenov et al., 1977; Belikovich et al., 1988; Hysell et al., 1996]. During the first stage, with the typical time $\tau_{\mathrm{d}}$, their intensity decreases exponentially at a $6-20 \mathrm{~dB}$ rate. Then, the decay slows down significantly. This pattern is much more pronounced during night at larger scales lower pump frequencies. As a rule, for the second (retarded) decay stage, the calculated effective diffusion coefficient is about $\mathrm{D} \approx(1-2) \times 10^{2} \mathrm{~cm}^{2} / \mathrm{s}$. This magnitude is significantly smaller than $\mathrm{D}_{\mathrm{a} \perp}$ and cannot be explained in terms of an ordinary diffusion process. It is suggested that the slow relaxation process can result from either the effect of natural plasma disturbances or nonlinear interactions between different spectral components of the artificial low-frequency turbulence [Hysell et al., 1996]. It should be noted that the characteristics of such retardation for decametric irregularities depends strongly on the position of the irregularities in the heated volume [Hysell et al., 1996].

\subsubsection{Temporal Evolution of Short-Pulse Pumped FAIs}

The temporal evolution of the scattered signal for a short-pulse, $\tau_{\mathrm{p}} \ll 1 \mathrm{~s}$, experiments has been analyzed in the case when the SSI intensity does not reach saturation [Frolov et al., 1997]. These experiments have shown that for $\tau_{\mathrm{p}}=50$ and $100 \mathrm{~ms}$ the scattered signal maximizes in $\sim 250 \mathrm{~ms}$ after the pulse ends. Thus, the SSI growth time greatly exceeds $\tau_{\mathrm{p}}$. For $\tau_{\mathrm{p}}=300 \mathrm{~ms}$, strong scattered signals are detected near the end of pumping or somewhat later. It has been found that the SSI 
growth time strongly varies from 0.1 to a few seconds in different pumping cycles; the longer time is observed for larger-scale irregularities.

These experiments demonstrate that $3 \mathrm{~m}$ irregularities remain for about $0.3-1 \mathrm{~s}$ after the pump switch-off. The relaxation of such non-saturated turbulence has larger decay rates as compared to that of the saturated turbulence, observed after a rather long pumping. The fast stage of the SSI relaxation lasts no more than $1 \mathrm{~s}$, then the decay rate decreases to that of the steady state conditions.

The experiments also show that the temporal evolution of the SSI is determined significantly by the pulse repetition period. Namely, after the pulse is off, $3 \mathrm{~m}$ irregularities continue growing for a longer time for shorter repetition periods. That is, the residual level of SSIs determines the level of energy stored in plasma before the intensive SSI development begins. Plausibly, this causes the well-known preconditioning during repeatable pulse duty cycles.

On average, the SSI intensity for $P_{0} \approx 100 \mathrm{MW}$ ERP begins to grow only within $\tau_{0} \approx 50-100$ $\mathrm{ms}$ and maximizes in $\sim 300 \mathrm{~ms}$, which is also the development time of the fast anomalous absorption. Based on the above results, three types of SSI relaxation regimes have been identified:

- the ordinary, ambipolar diffusion, regime, observed after long-time pumping;

- the rapid regime during the first stage of relaxation of the non-saturated turbulence;

- the slow regime, which follows the ordinary one.

The SSI relaxation features dependend on the anomalous absorption decay time, $\tau_{\mathrm{d}}(\mathrm{AA})$, and pulse duration, $\tau_{\mathrm{p}}$. Boiko et al. [1990] demonstrated that $\tau_{\mathrm{d}}(\mathrm{AA}) \approx 100-300 \mathrm{~ms}$ for $\tau_{\mathrm{p}}<3 \mathrm{~s}$, and the relaxation of absorption is determined by the decay of non-saturated irregularities with scale lengths $l_{\perp} \leq 3 \mathrm{~m}$, produced during the fast development stage. When the pulse duration exceeds 3 $\mathrm{s}$ and $10 \mathrm{~s}, \tau_{\mathrm{d}}(\mathrm{AA})$ increases, respectively, up to $10 \mathrm{~s}$ and even $30 \mathrm{~s}$, which corresponds to the decay time of the saturated decametric irregularities.

\subsubsection{Spectral Characteristics of SSIs}

Measurements of the backscatering from FAIs permit a determination of the transverse spatial spectrum $\Phi_{\mathrm{N}}\left(l_{\perp}\right)$ of the small-scale electron density fluctuations. It has been stated [Erukhimov, 1987; Frolov et al., 1997] that under steady state conditions and for $P_{0 \text { eff }} \approx 20 \mathrm{MW}$, the cross section of the IDV is about $10^{7} \mathrm{~m}^{2}, 10^{6} \mathrm{~m}^{2}$, and $10^{3} \mathrm{~m}^{2}$ for $l_{\perp} \approx 30 \mathrm{~m}, 10 \mathrm{~m}$ and $1 \mathrm{~m}$, respectively. Following Minkoff at el. [1974] the stationary spectrum $\Phi_{\mathrm{N}}\left(l_{\perp}\right)$ can be presented as $\Phi_{\mathrm{N}}\left(l_{\perp}\right) \propto l_{\perp} p_{0}$, where $p_{0} \approx 1-2$ for $l_{\perp} \approx 10-30 \mathrm{~m} ; p_{0} \approx 3$ for $l_{\perp} \approx 3-10 \mathrm{~m}$; and $p_{0} \approx 4-5$ for $l_{\perp} \approx 1-3 \mathrm{~m}$ [Erukhimov et al., 1987; Frolov et al., 1997].

The data presented have been obtained under optimum conditions when the PW frequency has been near but below the $F_{2}$ region peak frequency, far from gyroharmonics and measurements have been performed in evening or night hours when the IDV is located at heights $h \approx 230-270$ $\mathrm{km}$. 
If the temporal evolution of some spectral components is known, it is easy to calculate the time-dependence of the power index $p$ at the SSI development stage [Frolov, 2003]. The results of such calculations demonstrate that a maximum in the spectral intensity of the plasma density fluctuations $\Phi_{\mathrm{N}}\left(l_{\perp}\right)$ is attained for irregularities with scales $l_{\perp} \approx 3 \mathrm{~m}$ during the first several seconds of interaction between the HF powerful wave and ionospheric plasma (or when the maximum of SSI intensity is observed for irregularities with $l_{\perp} \leq 3 \mathrm{~m}$ ). Most likely, this phenomenon appears due to both the generation of SSI near the PW reflection height and the most effective generation of $3 \mathrm{~m}$ irregularities during the initial stage of pumping [Gurevich et al., 1995].

The generation of sufficiently intense small-scale irregularities with scales $l_{\perp} \leq 3 \mathrm{~m}$ at the initial stage of pumping explains the effect of $F$ region cross modulation [Frolov, 1981; Gurevich and Migulin, 1982]. It seems likely that this effect was first observed by Cohen and Whitehead [1970], but it was attributed to PW modifications in the lower ionosphere. This effect implies that the modification of the ionosphere by means of an amplitude modulated O-mode PW gives rise to an amplitude modulation up to $70-90 \%$ of diagnostic waves (both $\mathrm{O}$ - and X-polarization), sounding of the IDV in a wide-band frequency range of about several hundred $\mathrm{kHz}$ near the pump frequency. It has been found that: 1) this effect is observed only when the PW power $P_{0 \text { eff }} \geq 8 \mathrm{MW} \mathrm{ERP,2)}$ the typical time of decrease of diagnostic wave amplitudes is in agreement with the time of fast AA development, and 3 ) the $F$-region cross modulation is most pronounced if the PW power is square modulated by pump cycle of $3-10 \mathrm{~s}$ on/off.

In addition, it has been found that square wave modulation leads to the decrease in intensity of decameter-scale irregularities by a value of about 6-10 dB and to suppression of AA development when the modulation frequency is about $0.1-0.5 \mathrm{~Hz}$ [Belenov et al., 1977; Frolov, 2003]. Because this effect does not occur for meter-scale irregularities, such SSI properties can be used for their preferable generation.

In summary, during the first few seconds of pumping at $P_{0} \geq 10-20 \mathrm{MW}$ ERP, the meterscale striations dominate in the FAI spectrum. Particularly, $3 \mathrm{~m}$ irregularities become more pronounced in the SSI spectrum when the PW power is square-modulated with several seconds on/off period. Decameter-scale irregularities with stronger intensities than meter-scales develop later but they can be suppressed during the square wave modulation. Medium Scale Irregularities, that are more intense than Small-Scale Irregularities, reach their steady state within $10-20 \mathrm{~s}$ after PW switch-on. The growth of the LSIs, the most intense part of the FAI spectrum, lasts longer than $1-2$ minutes. Such temporal evolution of irregularities makes it possible to control their spectral characteristics varying both pump pulse duration and pulse repetition period. Evidently, the choice of particular timing for PW radiation depends strongly on such factors as ionospheric conditions, PW power and frequency, as well as on the PW reflection height and plasma density gradient in the interaction region. On the other hand, for forming only MSI and LSI in the spectrum of artificial irregularities, X-mode pumping has to be employed, when the suppression of SSI 
generation in the midlatitude $F_{2}$ region takes place. So, the above data demonstrate a possibility to control the spectral characteristics of the artificially generated irregularities that are important when utilizing the ionosphere as a natural plasma laboratory.

\subsubsection{Dependence of FAI Intensity on the Pump Power}

It is known that increasing the pump power causes a spatial expansion of the IDV in the horizontal plane [Erukhimov et al., 1978]. This determines a supplementary increase of the artificial field-aligned scattering intensity. To verify this hypothesis and to determine the actual dependence of the spectral intensity of plasma density fluctuations $\Phi_{\mathrm{N}}$ on $P_{0 \text { eff, different schemes }}$ for heating wave radiation has been employed using three antenna array sections for the coherent or incoherent mode for PW radiation. In the latter case, the antenna beam is about three times wider in the meridional direction in comparison with the case when all three sections are radiated in the coherent mode. Taking into account the change of IDV size in the meridional direction, it has been found that $\Phi_{\mathrm{N}} \propto P_{0 \text { eff }}{ }^{\xi}$, where $\xi \approx 0.4-0.8$ and $\xi \approx 0.7-1.2$ for $3 \mathrm{~m}$ and $10 \mathrm{~m}$ irregularities, respectively. Thus, there is a weaker dependence of SSI stationary intensity on pump power for its smaller scales or, in other words, the dependence $\Phi_{\mathrm{N}}\left(l_{\perp}\right)$ in the scale range $l_{\perp} \approx 3-$ $10 \mathrm{~m}$ can be intensified by an increase of the pump power.

Erukhimov et al. [1978] and Jones et al. [1983] show the existence of a hysteresis effect in the dependence of the artificial field-aligned scattering intensity, $I_{\mathrm{s}}$, on the PW power. $I_{\mathrm{S}}$ magnitude increases with increasing PW power, as has been discussed above, but $I_{\mathrm{s}}$ magnitude decreases at a slower rate at the branch of decreasing the PW power from its maximum level. Investigations performed in [Erukhimov et al., 1978] allow us to conclude that this effect is connected with threshold powers for generation of SSI: the threshold power for suppression of SSI generation is 2 - times smaller than the threshold power for their generation under "cold start" conditions. As a consequence, a hysteresis dependence of the IDV spatial size on the pump power takes place. It has also been stated that such a hysteresis effect is not observed for the spectral intensity $\Phi_{\mathrm{N}}\left(l_{\perp}\right.$,

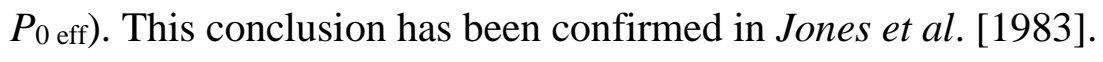

\subsubsection{Magnetic Zenith Effects}

Early heating experiments conducted at the Boulder facility, Colorado, USA in $1970-1973$ [Allen et al., 1974] revealed that the magnitude of anomalous absorption strongly depends on the geomagnetic aspect angle with the strongest absorption for probing wave rays propagating almost along geomagnetic field lines. Also, the displacement of the position of the most intense scattering from the ionospheric region illuminated by the central part of the HF beam to the southern periphery of the heated volume (to the magnetic field direction), was reported in [Belenov at al., 1977]. Notice that all the above mentioned experiments were performed using HF beams with halfpower width of $15^{\circ}-20^{\circ}$ directed at the geographic zenith. 
A direct demonstration of the characteristics of SSI generation when the PW beam is directed to the magnetic zenith has been performed at SURA. In these experiments the angle of the pump beam was scanned in a geomagnetic meridian plane from $32^{\circ}$ north to $32^{\circ}$ south relative to the vertical direction; in so doing the intensity of artificial backscatter from $3 \mathrm{~m}$ irregularities was determined. It was found that the most intense scattering is observed when the antenna beam declination is about $12^{\circ}-16^{\circ}$ off the vertical to the south taking into account that the Earth's surface, where the SURA antenna array is located, has inclination of $2^{\circ}$ towards to the south (for the SURA facility the magnetic field-aligned direction is about $19^{\circ}$ off the vertical to the south). The SSI intensity abruptly decreases for more southern angles and has a smoother fall-off for northern ones. In addition, an analogous angular dependence of the SSI intensity for decameter irregularities has been found in Uryadov et al., [2007].

The experimental data obtained may be explained by taking into account the facts that the condition of quasi-longitudinal propagation of an O-mode electromagnetic waves in the midlatitude ionosphere is satisfied more exactly when a wave vector $\boldsymbol{k}$ has a direction closer to the magnetic field lines at the height of the upper-hybrid resonance. For the SURA heating facility this condition for PW frequencies of about $5 \mathrm{MHz}$ is satisfied if the angle of the PW beam is about $14^{\circ}$ off the vertical to the south (or of about $12^{\circ}$ for the SURA antenna pattern taking into account the inclination of the Earth's surface). The abrupt decrease at larger southern angles is connected with the decrease of the PW reflection height below the height of the upper-hybrid resonance; its smoother fall-off is determined by worsening of the condition of quasi-longitudinal propagation of an O-mode electromagnetic waves. Therefore, the SURA antenna pattern is usually oriented at an angle of $12^{\circ}$ southward in the "magnetic zenith" direction for the PW, where, with allowance for refraction of radio waves in the ionosphere, the PW propagates along the geomagnetic field lines at the level of the upper-hybrid resonance.

Heating experiments at EISCAT have also reported that large-scale temperature enhancement depend strongly on the inclination angle of the incident EM pump wave relative to both the direction of electron density variation and the geomagnetic field direction; electron temperatures observed during geomagnetic field-aligned heating have been observed to be a factor of 2 or greater than those observed during vertically-aligned heating under similar conditions [Rietveld et al., 2003; Dhillon and Robinson, 2005; Honary et al., 2011].

Several mechanisms for the Magnetic Zenith effect have been proposed in the literature. They include the influence of the regular horizontal gradients [Rietveld et al., 2003], and multiple scattering of the pump wave on middle-size $(0.1-1 \mathrm{~km})$ irregularities [Zabotin and Kovalenko, 1999]. Gurevich et al. [2002] have suggested an explanation of the magnetic zenith effect based on self-focusing of the pump wave on striations. Leyser and Nordblad [2009] proposed an explanation based on a large-scale cavity stretched along the magnetic field line. Temporal dynamics of the magnetic zenith effect was investigated by Honary et al [2011]. It was reported 
that the temperature enhancement reaches its saturation level within $10 \mathrm{~s}$ after the heater is switched on. Time scales of 5-10 s are indicative of the development of small scale irregularities. Based on the fast manifestation of the MZ effect, a new theoretical explanation was proposed [Honary et al., 2011]. It was argued that some of the UHR modes trapped in striations are localized above the level where the plasma frequency coincides with the frequency of the pump wave due to the O-mode to Z-mode conversion process that can occur in the F-region for a narrow range of pump wave inclination angles. However, the greatest plasma perturbations have often been observed to occur not for pump waves inclined at the Spitze angle (at which conversion to the Zmode is theoretically most favorable), but for wave angles somewhere between the Spitze and the magnetic field directions. This suggests that the conversion process, and the "Z-mode window" for which conversion is likely to occur, may be modified by the presence of 2D inhomogeneities in the ionospheric plasma density close to the interaction region [e.g., Mishin et al., 2001; Gelinas et al., 2003].

A recently developed full-wave FDTD code [Cannon and Honary, 2015] was used to numerically explore the behavior of the O-to Z-mode conversion process and magnetic zenith effect was investigated for a variety of density profiles. These simulations [Cannon et al., 2016] show that large-scale linear density gradients, medium-scale duct-like density depletions and small-scale field-aligned irregularities were all found to affect the O-mode to Z-mode conversion process and consequently modify the position of the Z-mode window. This was shown to have a knock-on effect on the growth of thermal plasma perturbations due to the interaction of the heating wave and offers a potential mechanism behind several of the observed features of the magnetic zenith effect. Also, O-mode to Z-mode conversion can occur due to scattering off Langmuir cavitons [e.g., Eliasson, 2013].

\subsubsection{Unexplained UHF Radar Backscatter at the Magnetic Zenith}

A relatively new phenomenon, so far observable only at Troms $\emptyset$ because it is the only facility with an ISR capable of measuring along the geomagnetic field in the heated volume, is a large altitude extent enhancement of the incoherent scatter radar power above the HF reflection height which is observed only when the radar is pointing within about $0.5^{\circ}$ of the magnetic field. The enhancements are purely increases in the power of the natural incoherent scatter spectrum and appear as electron density increases. Senior et al. [2013] showed by two independent methods, however, that they are not electron density increases. These wide-altitude extent ion line enhancements (WAILES) are relatively common, very repeatable, and have been reported in many recent publications but, understandably, often interpreted as density enhancements [e.g. Blagoveshchenskaya et al., 2013, 2017; Borisova et al., 2017]. They also appear as plasma line intensity enhancements [e.g., Borisova et al., 2017]. 
Although no systematic study of WAILES has yet been made, from the various published observations it appears that the enhancements are strongest for X-mode heating, are anti-correlated with electron temperature enhancements and appear to be unrelated to decameter scale fieldaligned irregularities. The mechanism of the enhanced backscatter is not yet fully explained, but a possible explanation is that the UHF radar waves near grazing incidence are refracted and guided or ducted by large-scale field-aligned irregularities in the $F$ region so that their intensity does not fall of as $1 / r^{2}$ as they would in free space propagation would be quite valuable. Because there are many important implications of these results. It shows that the incoherent scatter radar-derived parameters may be wrong when pointing along the magnetic field in the presence of a certain class of field-aligned irregularities, which may possibly also occur naturally. The nature of these irregularities, and how they are produced by X-mode heating is not yet understood. Another feasible explanation for the WAILES can be ion acoustic waves excited by the heat flux instability (section 3.6.1), which can develop above the heating region.

\subsubsection{Gyroharmonic Effects Associated with FAIs}

In the previous sections, heater-induced irregularities' features have been considered for PW frequencies far away from an electron cyclotron harmonic frequency, $n f_{\text {ce }}(n$ is the harmonic number and $f_{\text {ce }}$ is the electron cyclotron frequency). In the first SEE measurements at the EISCAT heating facility, carried out in 1980s, it was found that SEE properties change significantly when the PW frequency lies within $100-200 \mathrm{kHz}$ of the frequency of electron gyroharmonic [Stubbe et al., 1984; Leyser, 2001]. Thereafter experiments, in which gyroharmonic pumping was used, were conducted regularly at both Troms $\varnothing$ and SURA heating facilities. Such investigations elaborated a method for experimental determination of an electron gyroharmonic frequency at the upper-hybrid resonance height based on SEE measurements through the frequency of down-shifted maximum (DM) suppression in SEE spectra when the condition of the double resonance is fulfilled (for more details see section 4.1).

This effect is connected with the suppression of upper-hybrid turbulence generation that manifests itself also as the suppression of SSI when the PW frequency approaches the gyroharmonic resonance frequency [Honary et al., 1999; Ponomarenko et al., 1999]. Note that the SEE method permits one to determine the frequency of the $4^{\text {th }}$ gyroharmonic with an accuracy of $\pm 5 \mathrm{kHz}$ and with higher accuracy for higher harmonics [Leyser et al., 1994]. In experiments [Ponomarenko et al., 1999] it has also been revealed that for a PW frequency slightly exceeding the $4^{\text {th }}$ gyroharmonic, scattering signals from heater-induced decameter irregularities show significant broadening of their spectra up to $5-10 \mathrm{~Hz}$ and the transition from broad to narrow spectra after the PW switch-off takes place in a very short time interval of about $50-70 \mathrm{~ms}$. The explanation of this effect has led to development of the theory of generation of very intensive artificial supra-small-scale irregularities of the plasma density with $l_{\perp} \approx 10-20 \mathrm{~cm}$, which are 
excited within the decameter irregularities when the PW frequency is close to or somewhat higher than a gyroharmonic frequency [Gurevich and Zybin, 2006].

Since 2004, several targeted experiments have been performed to study features of super small scale irregularities [Kagan et al., 2006; Frolov et al., 2012]. Scattered signals from decameter irregularities at frequencies $10-22 \mathrm{MHz}$ (from SSI with $l_{\perp} \approx 7-16 \mathrm{~m}$ ) have been received at two sites located near Kharkov (Ukraine) and Rostov-on-Don (Russia). Results from these experiments can be summarized as follow:

1) The scattered signal can be represented as a composition of its narrow-band and broadband components. The first component in its characteristics corresponds to the scattering observed at all PW frequencies, while the second (broadband) component of scattering has pronounced gyroharmonic properties and observed when $f_{0}$ is somewhat above $n f_{\text {ce }}$.

2) The maximum broadening of the scattered signal from heater-induced irregularities takes place when the PW frequency offset from the gyroharmonic was $\delta f_{m}=f_{0}-4 f_{\mathrm{ce}} \approx 20-60 \mathrm{kHz}$, exactly where the most intense generation of the Broad Up-shifted Maximum (BUM) emission component is observed, which is the most intense broadband SEE component observed in the upper sideband of the pump wave [Leyser et al., 2001; Frolov et al., 2001]). The value of $\delta f$ depends on conditions of the measurements. It is important to note that the spectrum broadening is observable even for $\delta f \cong 0$ where both the thermal (resonant) parametric instability and the SSI generation is suppressed, and where the fast BUM component is registered. It disappears only at $\delta f \approx-20 \mathrm{kHz}$. Above $\delta f_{\mathrm{m}}$, the spectrum width decreases gradually. The spectrum broadening also covers the region of the Broad Up-shifted Structure (BUS) generation.

3 ) The spectrum width for scattering from decameter artificial irregularities with $l_{\perp} \approx 10-20$ $\mathrm{m}$, in its steady state, can reach $10 \mathrm{~Hz}$ under optimal conditions of measurements (i.e. in the evening or night hours). The aspect-scattering surface passes through the "magnetic zenith" region for the PW with the most intense SSI and the PW frequency $f_{0}$ is only slightly below $f_{O} F 2$; ionospheric pumping has to be conducted at $P_{\text {eff }} \geq 50$ MW ERP.

4) The broadband component of the scattered signal develops together with decameter irregularities; according to precise measurements, the typical time of broadband component relaxation is about of $0.4-0.9 \mathrm{~s}$, during which a decrease in the intensity of this component is determined by a rapid narrowing of the received-signal spectrum at a rate of $1 \mathrm{~Hz}$ for $0.2-0.3 \mathrm{~s}$ [Frolov et al., 2012]. The obtained magnitude of the broadband component relaxation time is an order of magnitude greater than its value $0.05-0.07 \mathrm{~s}$ given in [Ponomarenko et al., 1999].

5) The dependence of the spectral broadening on the PW power for the "cold start" condition can be represented as $\Delta F \propto\left(P_{0 \text { eff }}\right)^{\alpha}$ with $\alpha \approx 0.5-0.8$. This power dependency illustrates a hysteresis effect. On the branch of the decrease of a frequency offset from the gyroresonance after $\delta f \approx 60 \mathrm{kHz}$ the spectrum width $\Delta F$ of the broadband scattering component has greater values than on the branch of its increase with the measurements started at $\delta f \leq 0$. As a result of that, if the 
increase of $\delta f$ starts with $\delta f<0$, for $\delta f \approx 0$ on the decreasing branch the spectrum remains notably broader than on the frequency increasing branch. Thus, the undisturbed (or weakly disturbed) values of $\Delta F$ for $\delta f \approx 0$ can be obtained only in the case where the previous plasma heating is conducted at negative $\delta f$.

A theoretical interpretation of the broadband component for the PW frequency of the same order of magnitude or slightly higher than the electron gyroharmonic frequency in the region of PW-plasma interactions, has been proposed in Gurevich and Zybin [2006]. According to the theory, this is due to very intense Supra-Small-Scale $\left(l_{\perp} \approx 10-20 \mathrm{~cm}\right)$ Irregularities (SSSI) of the plasma density, which are generated by the upper-hybrid and Bernstein waves locked within the decameter plasma-density irregularities. These waves are excited as a result of the development of a four-wave parametric instability of a high-power electromagnetic wave with O-mode polarization in the magnetized plasma when the PW frequency is slightly above the electron gyroharmonic frequency. Bernstein waves have a standing structure and large amplitude electric field. This leads to the supra-small-scale irregularity formation because of the striction (ponderomotive) pressure force.

The detection of intense SSSI with $l_{\perp} \approx 10-20 \mathrm{~cm}$ under conditions when the PW frequency slightly exceeds the gyroharmonic frequency in the region of interaction between a high-power $O$ mode radio wave and the plasma is currently one of the priority problems of experimental research in the field of the ionospheric plasma modification by high-power HF radio waves. The generation of such irregularities was predicted in Gurevich and Zybin [2006]. Clearly, the detection of SSSI requires the use of gigahertz radio waves. In particular, signals from the GPS/GLONASS navigation systems can be employed. The first such measurements have been performed in 2008 at HAARP with the PW frequency close to the third harmonic of the electron gyrofrequency [Milikh et al., 2008b].

The first detection of SSSI over SURA was made in 2010 [Frolov et al., 2012]. An example of the sounding of the heated volume by GPS signals is presented in [Frolov et al., 2017]. In this heating session the PW frequency equals of $5400 \mathrm{kHz}$ at $4 f_{\mathrm{ce}} \approx 5360 \mathrm{kHz}$, the pulsing of $\mathrm{HF}$ transmitters was $10 \mathrm{~s}-$ on, $10 \mathrm{~s}$ - off from 18:40 UT until 19:40 UT, the PW power was about 50 MW ERP, the antenna beam inclination was of $12^{\circ}$ to the south from the vertical. In the measurements characteristics of the slant total electron content (STEC) were determined. The trajectory of the ionospheric penetration point of a satellite used in these measurements is shown in Figure 4.6. During this session, the ionospheric penetration point for this satellite remains within the heated region (outlined at $0.1 P_{\text {eff }}$ in Figure 4.6) from 18:48 UT to 19:31 UT passing almost exactly through the center of the heated region, that is close to the "magnetic zenith" for the PW. The records of STEC and their detrended variations are presented in panel (b). The wavelet spectrum of STEC variations is shown in panel (c). In addition, the 5 minute period of the most intense STEC oscillations from 18:59 UT to 19:04 UT was expanded and shown in panel (d). 
Methods of processing of such experimental data are discussed in detail in [Milikh et al., 2008b; Kunitsyn et al., 2012; Najmi et al., 2014]. From data presented in panels (c) and (d) the existence of 20-s STEC variations is clearly seen, the period of which coincides with the PW on/off timing.

This experiment allows us to conclude that, when the PW is switched on, an increase in STEC by $0.02-0.03$ TECU $\left(1\right.$ TECU $\left.=10^{16} \mathrm{el} / \mathrm{m}^{2}\right)$ was observed in the region near the PW "magnetic zenith". These data permit one to estimate the typical time of increase in STEC as $2-5 \mathrm{~s}$ which, in general, coincides with the typical rise time of decameter irregularities; in this case the time of its decrease does not exceed $1 \mathrm{~s}$, which is much less than the relaxation time of decameter irregularities and corresponds to the relaxation time of the broadband scattered component discussed above. The latter can be considered as a circumstantial evidence of SSSI detection. Following the procedure elaborated in [Milikh et al., 2008b; Najmi et al., 2014], it can be found that SSSI density variations $\Delta N / N$ has to be about $2 \%-3 \%$. Results of such an estimation is in a good agreement with the results obtained at the HAARP facility taking into account higher PW powers of $1-2 \mathrm{GW}$ used for pumping.

\subsubsection{Concluding Remarks}

In this part of the review the basic features of heater-induced artificial field aligned irregularities have been presented and discussed at all stages of their evolution in accordance with scale-length, PW frequency and power, and ionospheric conditions. Experimental observations resulted in the development of the empirical model of SSI when the pump wave frequency is outside the gyroharmonic frequency ranges [Frolov et al., 1997]. Further elaboration of the model has been made in Sergeev et al. [2017], where a height dependence of SSI features has been included.

The SSI model has been used by Shvarts et al. [1995] to calculate the temporal evolution of the broad continuum (BC) emission component in the SEE spectrum and evolution of diagnostic SEE (DSEE) observed after a long-term pumping, as well as to explain the dependence of the SEE features on the PW power and frequency [Sergeev et al., 1999]. SEE measurements can be utilized for the study of some of the SSI features. After investigation of the fundamental SEE features, which were summarized by Stubbe et al. [1984; 1994], Stubbe and Hagfors [1997], Frolov [2001], Leyser [2001]. SEE has become a very useful tool to study HF-induced nonlinear processes in the ionospheric plasma.

It is important to emphasize that SEE measurements are carried out directly without a need for additional electromagnetic waves for probing, such as used in radars. SEE measurements show that thermal emission components $\left(\mathrm{NC}_{\mathrm{th}}, \mathrm{DM}\right.$ and $\left.\mathrm{BC}\right)$, which are generated due to the thermal parametric instability development, are more intense in a $\mathrm{PW}$ frequency rage $f_{0} \leq 6 \mathrm{MHz}$, in close agreement with the theoretical consideration [Gurevich et al., 2002]. 
Based on SEE features, a two-wave scheme of pumping has been developed in Frolov et al. [1994]. It comprises two different waves. An O-mode pump wave, having diagnostic properties (diagnostic wave, DW), to stimulate the SEE, named as diagnostic SEE (DSEE), which in turn is used for diagnosing the artificial ionospheric turbulence. In the measurements, the DW power is chosen to induce an unsaturated turbulence at a rather low level, which can flexibly respond to any additional external actions. The second wave (pump wave, PW) can have $\mathrm{O}$ - or $\mathrm{X}$-mode polarization. This wave is used to create additional ionospheric disturbances which can influence the DW-induced turbulence to manifest itself in changes of SEE characteristics. The scheme of additional pumping makes it possible to distinguish between the influence of different factors on the generation and evolution of artificial turbulence by varying PW parameters such as its polarization, power, frequency, and timing.

Some illustrations of use of the two-wave pumping scheme are presented in [Frolov et al., 2016], in which daily variations of DSEE features and their connection with SSI characteristics are considered; features of Langmuir and upperhybrid plasma turbulence evolution are studied in Sergeev et al. [1998], short-pulse pumping of the ionospheric plasma has been employed to study transport processes in the upper ionosphere [Sergeev et al., 2017], and artificial ionospheric turbulence features have been investigated when a

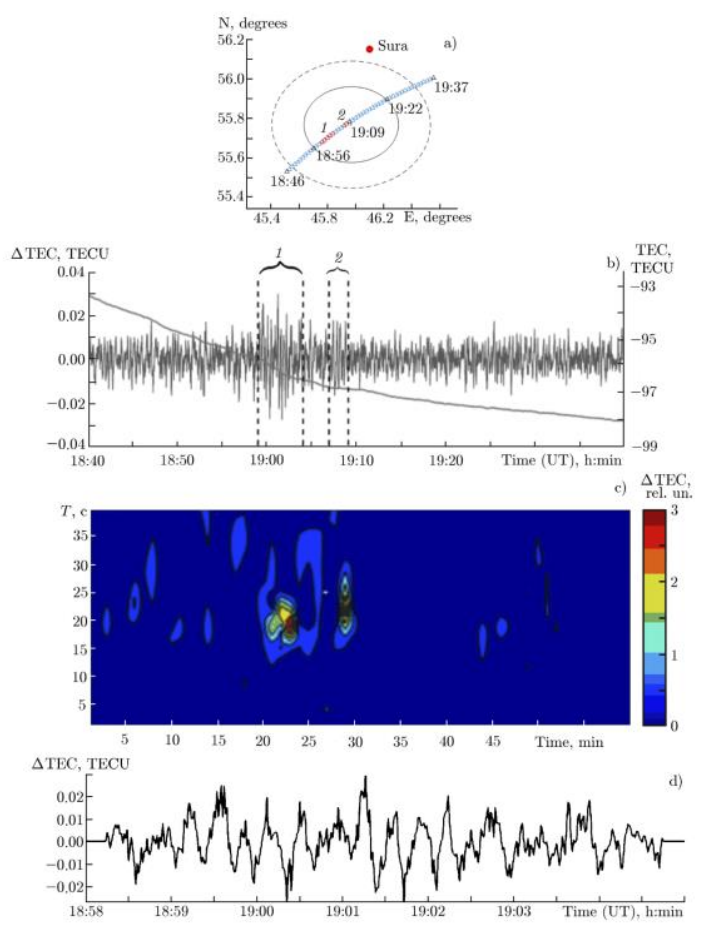

Figure 4.6. The results of measurements on August 21, 2011. Panel (a) shows a projection of the satellitereceiver ray trajectory on the heater radiation pattern in geographic coordinates. Panel (b) shows the TEC and its variations during a transit of the satellite above the disturbed ionospheric region, Panel (c) shows the results of the wavelet analysis of TEC variations, and panel (d) shows a zoomed oscillogram of TEC in the magnetic zenith region (region 1, in panel a) [Frolov et al., 2017].

PW with X-mode polarization is used for ionosphere modifications [Frolov et al., 2014]. All these investigations have shown that SEE provides a rather sensitive method to study the turbulence features. To complete the consideration of SSI features, it should be mentioned that at SURA such irregularities were not generated by X-mode waves [Frolov et al., 2014], unlike in high-power Xmode experiments at the EISCAT facility [Blagoveshchenskaya et al., 2011; 2013; 2017].

\subsection{Ducts}

One of the significant nonlocal effects of $\mathrm{HF} \mathrm{O}$-mode heating is the creation of $\mathrm{O}^{+}$ion outflows observed by the EISCAT UHF ISR, DMSP spacecraft, and HAARP Digisonde sky map technique [Rietveld et al., 2003; Milikh et al., 2010a; Blagoveshchenskaya et al., 2011; Kosch et al., 2010; 
2014; Vartanyan et al., 2012] along with plasma density enhancements (artificial ducts) in the topside ionosphere measured onboard the DEMETER and DMSP spacecraft [Frolov et al., 2016; Milikh et al., 2008a; 2010a; Rapoport et al., 2010; Markov et al., 2010; Vartanyan et al., 2012]. During satellite overflight experiments, the heating facility was usually turned on 10-20 min before the predicted satellite crossing the facility magnetic flux tube at the closest distance $\Delta \mathrm{R}_{\mathrm{c}}$ from the center (MZ). Overall, the artificial ducts and ion outflows have been observed at $\Delta \mathrm{R}_{\mathrm{c}}$ ranging from 10 to $150 \mathrm{~km}$.

\subsubsection{DEMETER Observations over SURA}

An extensive program was performed with SURA amounting to a total of $\sim 200$ satellite passes over the 6 years of DEMETER operati-ons. The main goal of this program was to specify the development of artificial ducts and their effect on VLF wave propagation using plasma and wave measurements from DEMETER. During these experiments, O-mode HF waves were injected either at vertical or $12^{\circ}$

South to benefit from the MZ effect.

Overall, only nighttime (21:3022:30 LT) experiments resulted in observable effects. The lack of noticeable effects during daytime (09:30-10-30 LT) has been attributed to the absorption of the pump-waves in the lower ionosphere and defocusing when $f_{0}$ exceeds the $F_{2}$-peak plasma frequency foF 2. For the nighttime experiments, the conditions necessary for successful observations are

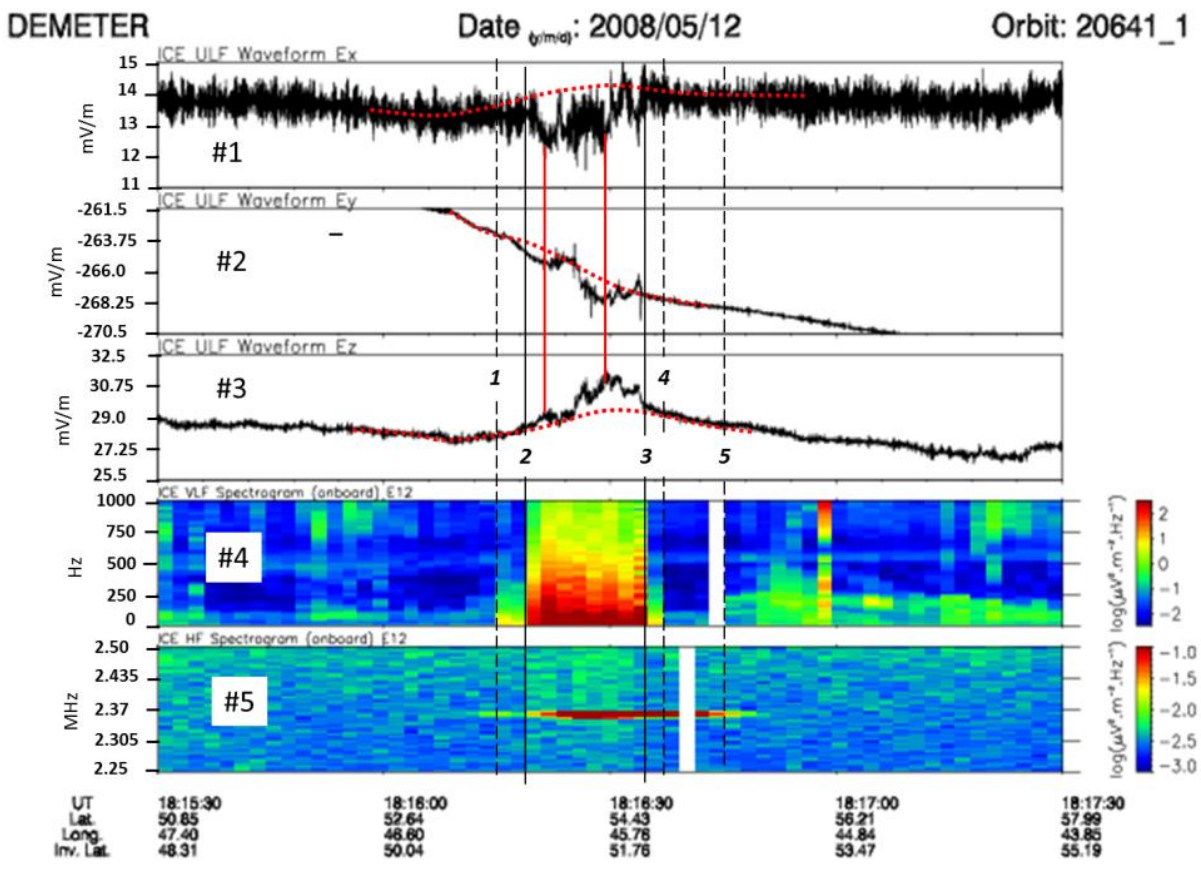

Figure 4.7. Electric Field Measurements during Orbit 20641_1 over SURA. Top 3 plates $\# 1,2,3$ : DC-ULF E-field components in DEMETER frame of reference $E_{x}$ nadir, $E_{y}$ perpendicular to orbit plane, eastward, $E_{z}$ opposite to S/C velocity, southward. The estimated base lines of the DC E-field components in absence of SURA emission are shown by the red dotted curves. Indicated by solid red lines, two maxima of the E-field disturbance are observed, the first one with $\sim 1 \mathrm{mV} / \mathrm{m}$ on all three components, the second one with $1.5 \mathrm{mV} / \mathrm{m}$ on $E_{x}$ and $2.5 \mathrm{mV} / \mathrm{m}$ on both $E_{y}$ and $E_{z}$. Plate \#4: ELF electrostatic turbulence spectrum along E12 $\sim$ Ey showing a nearly constant level between $\mathrm{T} 2$ and $\mathrm{T} 3(\sim 32 \mathrm{~s})$ a reduced level on the edges of the main events in intervals [T1-T2] $\sim 8 \mathrm{~s}$ and [T3-T4] $\sim 4 \mathrm{~s}$. The large signal just before 18:17:00 is due to lightning. Plate \#5: HF component along E12 due to the pump signal (see text), starting simultaneously with the electrostatic turbulence but extending further by $\sim 20$ s till T5. 
[Frolov et al., 2016]: (1) ERP $\geq 40 \mathrm{MW}$, (2) the heating frequency, $f_{0}$, smaller than $f_{o F}$ by 0.5 $0.7 \mathrm{MHz}$, and (3) $\Delta \mathrm{R}_{\mathrm{c}} \leq 50 \mathrm{~km}$. For $f_{0} \leq f o F 2-1 \mathrm{MHz}$, the pump wave energy is deposited far below the F-peak altitude where ion-neutral collisions slow down ion upflows and thus the duct formation on the topside.

Figures 4.7 and 4.8 show the SURA-DEMETER artificial duct and ion outflow observations in the quiet- night-time ionosphere with foF2 $\approx 5 \mathrm{MHz}$ on 12 May 2008 . The heater was turned on during 18:05 - 18:20 UT (22:05 - 22:20 LT) injecting a $4.3 \mathrm{MHz}, 80 \mathrm{MW}$ ERP ordinary wave at $12^{\circ}$ South. The calculated reflection altitude $h_{0}$ was $220 \mathrm{~km}$. DEMETER was in the survey mode thus providing only low time resolution plasma and wave measurements shown during a 2 min interval centered at $T_{c}=18: 16: 28 \mathrm{UT}$ when $\Delta R_{c}$ was about $21 \mathrm{~km}$.

In Figure 4.7 ( $4^{\text {th }}$ frame), the reddish part of the frequency-time

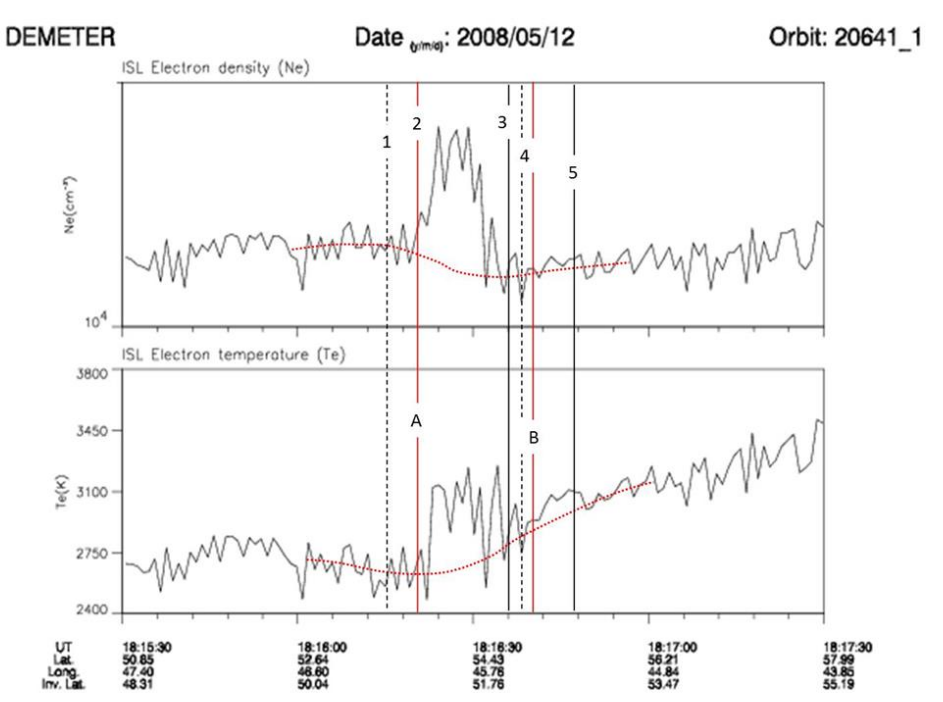

Figure 4.8. Thermal electron measurements during orbit 20641_1 over SURA. Top panel: electron density $\mathrm{N}_{\mathrm{e}}$, Bottom panel: electron temperature $\mathrm{T}_{\mathrm{e}}$. The estimated base line in absence of disturbance are shown by red dotted lines. The various time lines $\mathrm{T} 1 \ldots \mathrm{T} 5$ and $\mathrm{T}_{\mathrm{A}}, \mathrm{T}_{\mathrm{B}}$ indicated in Figures D01 are reproduced. The $\mathrm{N}_{\mathrm{e}}$ increase is in accordance with the increase of $\mathrm{N}\left(\mathrm{O}^{+}\right)$since $\mathrm{O}^{+}$is the major ion species and the electron heating occurs simultaneously with the electrostatic turbulence enhancement. Adapted from Frolov et al. [2016]. spectrogram of the ELF electric field component perpendicular to the orbit plane indicates that the most-affected area extends $\sim 60 \mathrm{~km}$ along the orbit path with abrupt edges indicated by vertical solid lines. The ELF waves in the adjacent regions (indicated by vertical dashed lines) have much lower intensity. The HF spectrum at $\sim 2.37 \mathrm{MHz}$ (bottom) intensifies at the southern edge and continues northward by $\sim 130 \mathrm{~km}$. Since the ICE passband is limited to $3.175 \mathrm{MHz}$, this signal results from aliasing of the pump wave digitized at $6.66 \mathrm{MHz}$, i.e., $f=6.66-f_{0}$. Three components of the DC-ULF electric field (top three frames) are also enhanced up to $2 \mathrm{mV} / \mathrm{m}$ ( $E_{y}$ and $E_{z}$ ) mainly in the northern part of the region of the enhanced ELF turbulence.

Figure 4.8 shows that the density of $\mathrm{O}^{+}$ions is enhanced by $55 \pm 10 \%$ between 18:16:21 and 18:16:40 UT, i.e., along about $140 \mathrm{~km}$ along satellite path which is greater than that of the enhanced ELF turbulence but close to that of the enhanced HF signal. Coincident with the plasma density enhancement, the electron temperature $T_{e}$ also increases by $\sim 250 \mathrm{~K}$ in the center of the duct, while the ion temperature does not show any significant variation. The $\mathrm{H}^{+}$and $\mathrm{He}^{+}$ion 
densities (not shown) behave quite differently. Namely, while $n_{H^{+}}$increases by about $50 \%$ only at the duct's northern edge, $n_{H e^{+}}$decreases by order of magnitude inside the duct.

The coincident variations of the $\mathrm{O}^{+}$speed angles (not shown) indicate that ion drifts associated with the heating occur mainly on the northern side of the duct, collocated with the enhanced DC-ULF electric field. Their field-aligned upward velocity is about $200 \mathrm{~m} / \mathrm{s}$, while the perpendicular to orbit plane component is $\sim 100 \mathrm{~m} / \mathrm{s}$ relative to that outside the duct. The $n_{e}$ and $T_{e}$ profiles in Figure 4.8 display modulations of up to $\sim 30 \%$ in $n_{e}$ and $\sim 15 \%$ in $T_{e}$ at $2-3 \mathrm{~Hz}$ in the duct. Albeit similar modulations of much lower amplitudes are also observed outside the duct, it seems likely to infer from these modulations that field-aligned filaments of $\sim 20 \mathrm{~km}$ transverse scale-size are generated in the heated volume. It is worth of note that in several SURA-DEMETER experiments coincident with injections of powerful VLF electromagnetic waves from the nearby VLF transmitter and artificial ducts served as a waveguide for VLF whistler waves [Markov et al., 2010; Rapoport et al., 2010].

\subsubsection{DMSP and DEMETER Observations over HAARP}

Observations of HF-induced ducts and ion outflows were performed at HAARP during 20072010 experimental campaigns [Milikh et al., 2008; 2010a; Vartanyan et al., 2012] in excess of 70 DEMETER and DMSP successful overflights. After at least $20 \mathrm{~min}$ off (the "cold" start), the HAARP heater operated at the maximum 3.6 megawatt (MW) power available, O-mode polarization, and the radio beam directed into MZ. The HF heating frequency during daytime experiments was chosen near $f o F 2$, while during nighttime it was 2.8 or $2.85 \mathrm{MHz}$ i.e., close to the second electron gyroharmonic in the F2-region. The modified ionosphere was sensed by instruments aboard the DEMETER and DMSP spacecraft available at the time of the experiment. The satellite observations were complemented by ground based diagnostics provided by the HAARP Digisonde and the Kodiak radar. The Digisonde operates in a regular mode producing ionograms to choose a proper heating frequency, and in skymap mode for carrying out bottomside diagnostics of the heated region.

In the majority of experiments, the spacecraft crossed the heated spot with a 10-15 min delay relative to the start of heating. In two SURA-DMSP and HAARP-DMSP experiments on 23 August 2007 and 10 October 2007 and on 11 November 2009 and 05 October 2010 the crossing occurred with a delay of $\sim 5 \mathrm{~min}$ and $3 \mathrm{~min}$, respectively. Figure 4.9, adapied from [Milikh et al., 2010a], shows DMSP overflights on 4 and 10 February 2010 with 12 and 6 min delay (left frames), respectively, and on 11 November 2009 (right). It is evident that the ducts have already been formed before the crossings, which requires the ion upward speed of the order of $3 \mathrm{~km} / \mathrm{s}$.

The duct regions extend by $\leq 100 \mathrm{~km}(\leq 15 \mathrm{~s})$ around $T_{m z}$, which is of the order of the diameter of the half power HF beam width at the satellite altitude of $850 \mathrm{~km}$. The spatial profile of the ion outflow is similar to that of the local ion density. At the same time, ion outflows lead to ejection 
of the light $\mathrm{H}^{+}$and $\mathrm{He}^{+}$ions, thereby increasing the fraction of $\mathrm{O}^{+}$ions. On 4 February and 31 June 2010, concurrent measurements of Doppler shift of oblique echoes reflected from the irregular heated spot determined the outflow speed increasing from $\sim 40$ to $70 \mathrm{~m} / \mathrm{s}$ in the altitude range 270 $370 \mathrm{~km}$.

Overall, the relative ion density increase, $\Delta n / n$, in the duct ranges from $\sim 5$ to 75 per cent, while the field-aligned ion speed change relative to the background, $\Delta V$, by 20 to $400 \mathrm{~m} / \mathrm{s}$ upward. Larger values of $\Delta n / n$ correspond to greater $V_{u p}$, though deriving a quantitative relation is hampered because $\Delta \mathrm{R}_{\mathrm{c}}$ significantly varied in different overflights. As a rule of thumb, the lower the reflection height or the F2-peak altitude below $200 \mathrm{~km}$ and greater $\Delta \mathrm{R}_{\mathrm{c}}$, the smaller $\Delta V$. The daytime ducts are of smaller relative amplitude and narrower than the nighttime ducts. The width of nighttime ducts is greater than 100 (up to 200) $\mathrm{km}$ in the N-S direction versus $70-80 \mathrm{~km}$ of the daytime ducts. Density perturbations in the nighttime ducts are greater than $20 \%$ (up to $70 \%$ ), while the daytime ducts are weaker than 15\% [Vartanyan et al., 2012], consistent with the absence of daytime ducts for $\leq 200 \mathrm{MW}$ ERP at SURA.
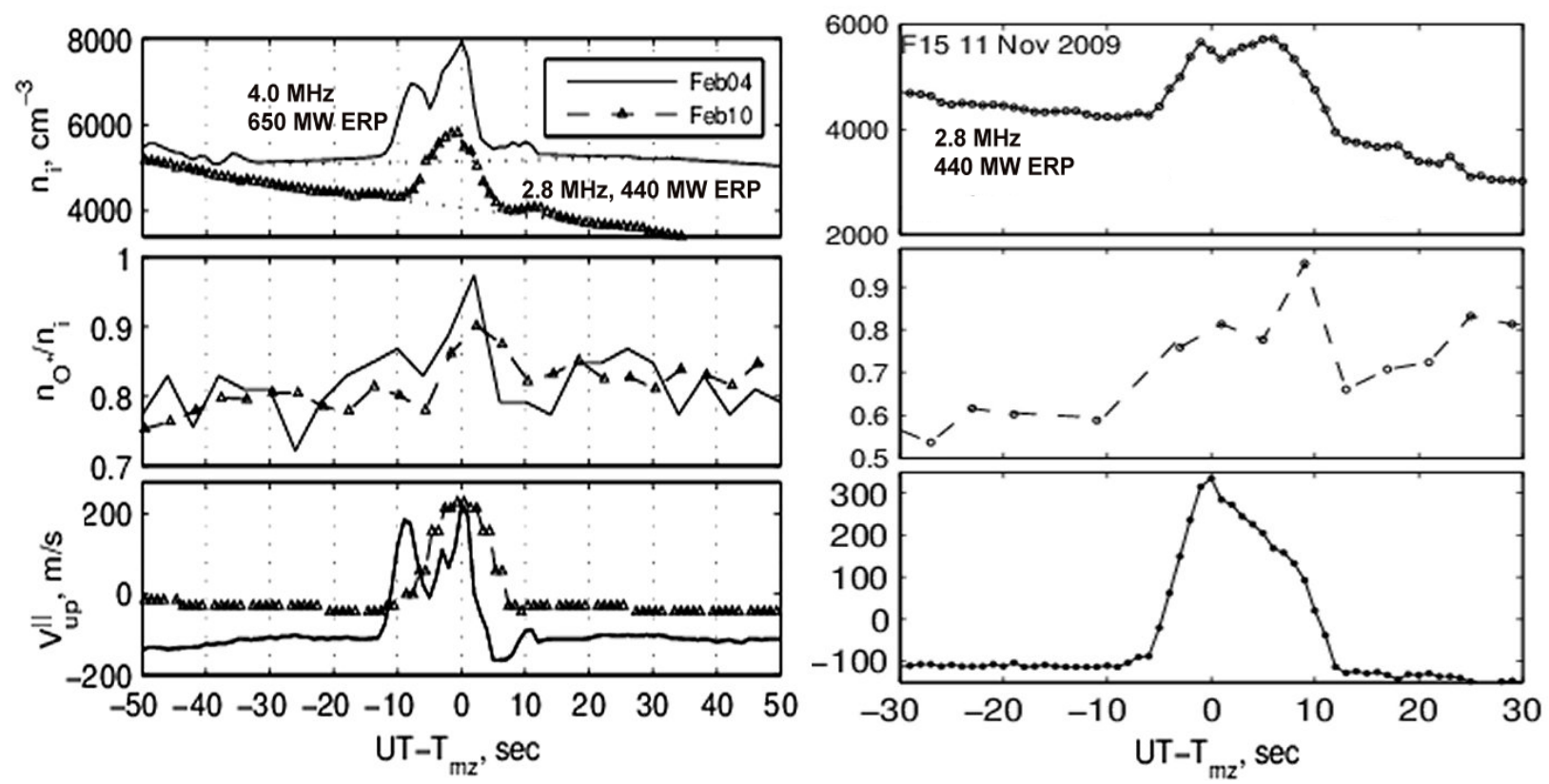

Figure 4.9. (Top) Total ion densities averaged over $1 \mathrm{~s}$, (middle) fractions of $\mathrm{O}^{+}$ions, and (bottom) 1 -s average of upward field-aligned ion velocities vs. time. The time axis is centered on the crossing of the MZ. The heating frequency and ERP are indicated. Adapted from [Milikh et al., 2010a].

\subsubsection{Numerical Modeling of Artificial Ducts}

As follows from general considerations, electron heating inside a thin, $L_{\|} \ll L_{T}=\lambda_{e} \delta_{i l}^{-1 / 2}$ (section 3.6.3), layer at an altitude $h_{T}$ increases the electron pressure around $h_{T}$ over the distance $\sim L_{T}$. The electron pressure imbalance leads to field-aligned ion flows due to the ambipolar electric field. Extensive simulations have been performed [Milikh et al., 2010; 2012; Vartanyan et al., 2012] by the SAMI2 code with a simple "hot brick" electron heating source imposed. That is, a 
Gaussian source $v_{e} W_{\text {tot }}$ (eq. 34) is centered at $h_{T}$, with the horizontal cross section of the HF beam at half maximum and $L_{\|} \sim 10 \mathrm{~km}\left(<L_{T}\right)$. The actual value of $L_{\|}$and a particular shape of the source are insignificant, as the fast heat transport will smooth out any sharp $T_{e}$ gradients well before the density profile starts changing. Only the total deposited energy matters. The simulations use the anomalous absorption mechanism caused by the HF-excited UH waves coupled with field-aligned density irregularities, so that $h_{T} \simeq h_{u h}$. Possible effects of accelerated suprathermal electrons (section 3.7) are neglected.

Figure 4.10 exemplifies SAMI2 simulations for the nighttime (a) and daytime (b) HAARP-DEMETER experiments, with different peak heating rates corresponding to the absorption efficiency $\sim(4-10) \%$. The observed fine structure of the artificial ducts could be caused by large scale irregularities induced by the HF heating. It is seen that the steady-state solutions are consistent
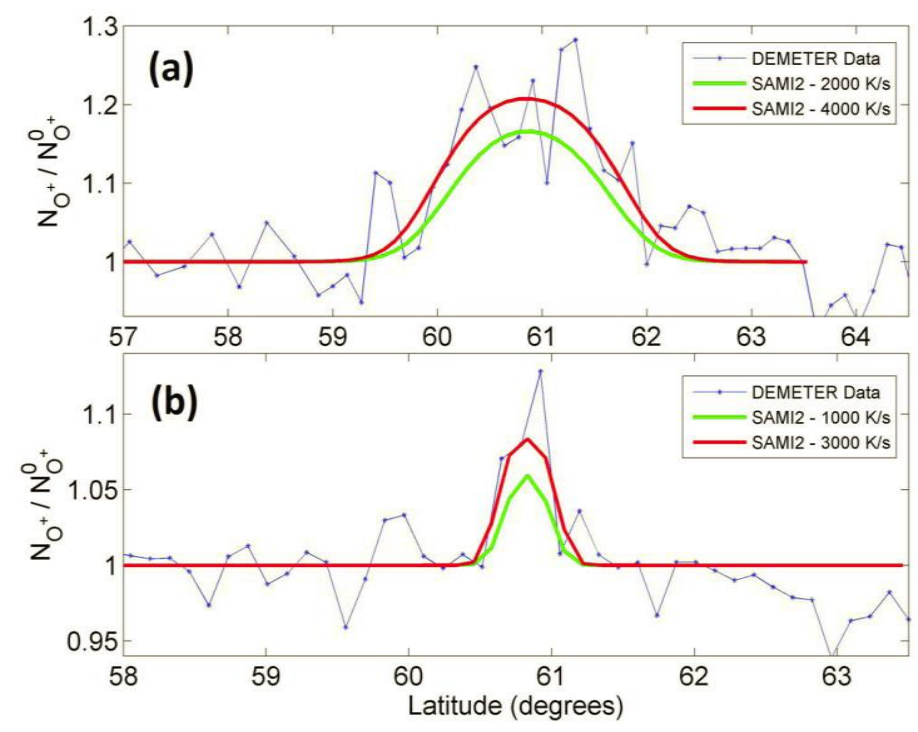

Figure 4.10. (a) Relative perturbations of the $\mathrm{O}^{+}$density on 21 October 2009 (connected points) along with SAMI2 model results (solid lines). (b) Observations and modeling for 7 November 2010 [Vartanyan et al., 2012]. with the observations reproducing the difference between the daytime and nighttime ducts. However, the SAMI2 simulations with moderate peak heating rates up to $5000 \mathrm{~K} / \mathrm{s}$ do not explain the fast appearance of artificial ducts and $\mathrm{O}^{+}$ion outflows in the topside ionosphere.

Kosch et al. [2010; 2014b] have shown that in order to match the observations of the HFinduced ion outflows from the EISCAT UHF ISR, a 1-2 $\mu \mathrm{V} / \mathrm{m}$ downward electric field is needed in addition to the electron pressure gradient. The latter, however, does not explain the fast timescale as the average upward speed does not exceed $\sim 0.5 \mathrm{~km} / \mathrm{s}$. So far, this problem is not understood and, likely, requires a more detailed consideration of the initial stage of the upward heat transfer including suprathermal electrons.

\subsection{Optical Emissions}

\subsubsection{Artificial Aurora}

Artificial optical emissions, with the same wavelengths as may be observed in auroras, appear when the pump-induced accelerated electrons acquire enough energy for collisional excitation of the surrounding neutral species, which are mostly atomic oxygen and molecular nitrogen in the Eand F-region. Ionization of both neutral species may occur, as observed by their unique optical signatures. As a general rule, the $630 \mathrm{~nm} \mathrm{O}\left({ }^{1} \mathrm{D}\right)$ emission is the brightest (typically $50-100 \mathrm{R}$ ), 
followed by $557.7 \mathrm{~nm} \mathrm{O}\left({ }^{1} \mathrm{~S}\right)$ (typically $\left.10-20 \mathrm{R}\right), 777.4 \mathrm{O}\left({ }^{5} \mathrm{P}\right)$ or $844.6 \mathrm{~nm} \mathrm{O}\left({ }^{3} \mathrm{P}\right)$ (typically 10 $20 \mathrm{R}$ ), and $427.8 \mathrm{~nm} \mathrm{~N}_{2}{ }^{+}$(typically $5-10 \mathrm{R}$ ). This corresponds approximately to the threshold excitation energies of 2, 4.2, 9, 11 and $18.6 \mathrm{eV}$ for $630,557.7,777.4,844.6$ and $427.8 \mathrm{~nm}$, respectively, and is the convolution of the accelerated electron and neutral excitation cross-section energy spectra. More recently, Mutiso et al. [2008] detected the $732 \mathrm{~nm} \mathrm{O}{ }^{+}$and $799 \mathrm{~nm} \mathrm{O}\left({ }^{3} \mathrm{D}\right)$ emissions with threshold excitation energies of 18.6 and $12.5 \mathrm{eV}$, respectively. Pump-induced optical emissions are for the most part sub-visual to the human eye, with some rare exceptions, and are mostly produced by the supra-thermal energy component of the electron population, as discussed below. It is noted that ground-based optical observations have been limited to wavelengths of $400-850 \mathrm{~nm}$ because of the available technologies, and any UV emissions generated in the thermosphere would be absorbed by the stratospheric ozone layer.

The optical response depends on the pump power and polarization, frequency relative to the electron gyro-harmonic frequency and the beam pointing direction relative to the magnetic field direction, as discussed below. The great majority of successful optical experiments were performed using O-mode polarization of the pump beam. However, against conventional wisdom, recent work using $\mathrm{X}$-mode polarization has also produced optical emissions, as presented below.

The main focus here will be on the period 2007 to the present. Kosch et al. [2007a] reviewed early optical work performed at the EISCAT and HAARP facilities whereas Kagan et al. [2006] presented early optical experiments at the SURA facility. These are briefly summarized here:

At SURA, the magnetic dip angle is $29^{\circ}$

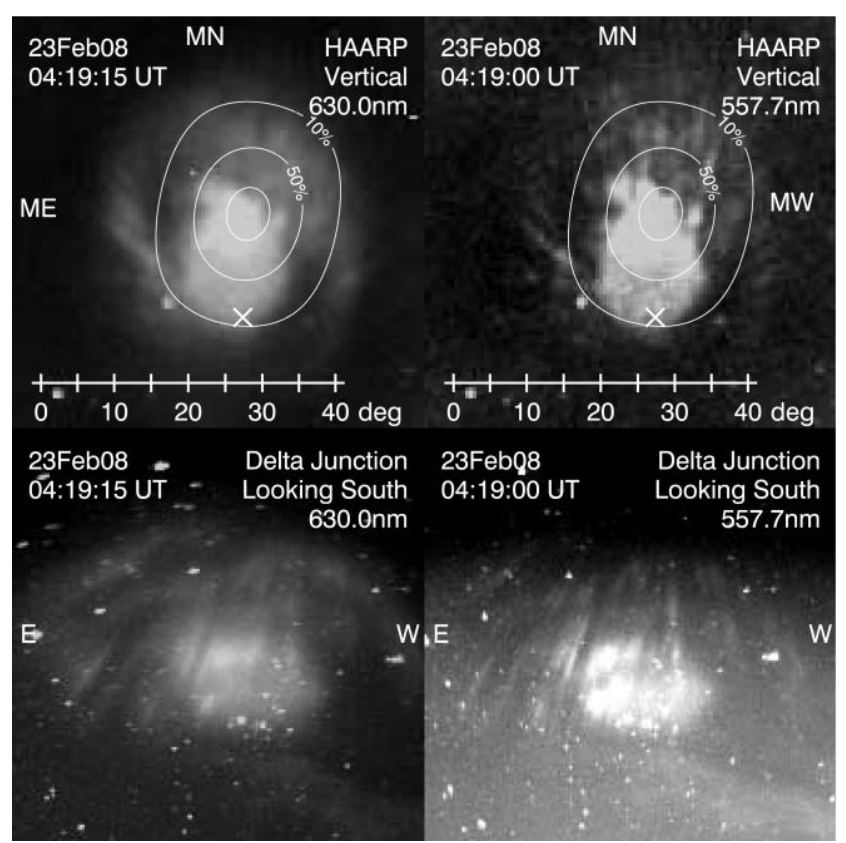

Figure 4.11. Optical images from the HAARP site looking up (top) and from Delta Junction $160 \mathrm{~km} \mathrm{~N}$ of HAARP looking obliquely $\mathrm{S}$ at $\sim 45^{\circ}$ elevation (bottom). White lines show contours of the vertical HAARP transmitter beam at 10\%, 50\%, and 90\% full ERP (from [Pedersen et al., 2009]).

approximately south of vertical. Kagan et al. [2006] found that the $630 \mathrm{~nm}$ optical emission occurred within the beam when pumping vertical but was displaced towards the magnetic zenith by $1-2^{\circ}$ when pumping $12^{\circ}$ south of vertical. Kagan et al. [2006, and references therein], reported first observations of the $557.7 \mathrm{~nm}$ from sporadic E-layers near $110 \mathrm{~km}$ altitude, and red $\mathrm{OH}(9-3)$ Meinel optical emission presumably from 80-85 km altitude, for vertical pumping.

Kosch et al. [2007a] gave a brief summary of early pump-induced optical observations since the 1970s at mid- and low latitudes from Russia and the USA. Is was not until early 1999 that 
similar unambiguous observations were made at high latitudes, notably at the EISCAT and HAARP facilities with magnetic dip angles of $13^{\circ}$ and $15^{\circ}$, respectively. Kosch et al. [2007a, and references therein] first reported that the optical emission at EISCAT was significantly displaced towards the magnetic zenith for vertical beam pointing, and sometimes appeared outside the $-3 \mathrm{~dB}$ locus of the pump beam. Subsequent experiments at EISCAT and HAARP confirmed the initial observation with the maximum optical response always in the magnetic zenith direction. The socalled "magnetic zenith effect" gave an optical gain exceeding an order of magnitude compared to other directions and occurred for all wavelengths.

The peak optical emission intensity appears to come of order $20 \mathrm{~km}$ below the pump wave reflection altitude [Gustavsson et al., 2008]. Mostly, the optical emission appears as blob of order $20 \mathrm{~km}$ across and is significantly elongated along the magnetic field line. Pump-induced optical emissions have been observed with a field-aligned extent up to $200 \mathrm{~km}$ [Kosch et al., 2007c; Pedersen et al., 2008]. With the HF reflection altitude typically around $200-250 \mathrm{~km}$ altitude in the F-region, optical emissions may appear from $\sim 150$ up to $\sim 350 \mathrm{~km}$ altitude as the accelerated electrons stream along the magnetic field line.

Small-scale spatial structuring, with irregular features down to $1 \mathrm{~km}$ horizontal size, was also observed and will be discussed further below. Unstable ring-like structures around the edge of the pump beam, which descended in altitude and collapsed into blobs over time, could be formed but these were extremely sensitive to the beam pointing direction (only $9^{\circ}$ south of vertical at EISCAT). This has subsequently also been observed at HAARP [Pedersen et al., 2009b].

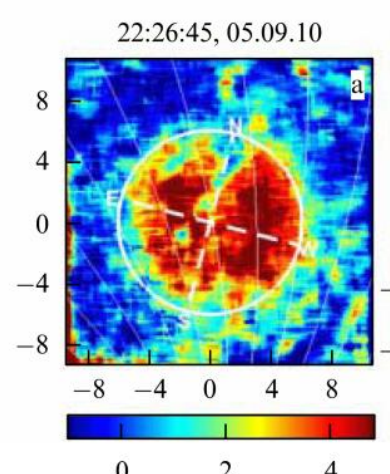

Figure 4.12. Optical images obtained on 03-05.09.2010 (a-c) and 15.03.2010 (d) in the night sky at the end of 2 minute quasi-continuous radio emissions from the SURA facility [Grach et al., 2016].

EISCAT incoherent scatter radar observations showed large electron temperature increases, typically up to $3500 \mathrm{~K}$, but these enhancements decreased significantly for beam pointing away from the magnetic zenith [Rietveld et al., 2003]. The electron temperature enhancement cannot explain the observed optical emissions for a thermalized plasma, hence the photons must come from a supra-thermal component of the accelerated electron energy spectrum. The first observation of $427.8 \mathrm{~nm}\left(\mathrm{~N}_{2}{ }^{+}\right)$occurred at EISCAT [Holma et al., 2006], proving that pump-induced ionisation was possible. 
Stepping the pump wave frequency at EISCAT through a gyro-harmonic greater than the second clearly showed a minimum in optical emissions, electron temperature enhancements and 10-m scale field-aligned plasma irregularities (striations), thereby demonstrating the association and importance of Upper-Hybrid Resonance (UHR) for these phenomena [Kosch et al., 2002]. However, evidence for Langmuir Turbulence (LT) accelerating electrons was also found [Ashrafi et al., 2006]. Pumping on the second electron gyro-harmonic frequency at HAARP was found to greatly increase the optical emissions (by about an order of magnitude) and striations compared to any other frequency. Optical data revealed an asymmetry about the gyro-harmonics, namely, the production of optical emissions and striations was significantly greater when the pump frequency was just above, compared to just below, the gyro-harmonics [Gustavsson et al., 2006]. Estimates of the pump-induced electron energy spectrum, from a combination of incoherent scatter radar and multi-wavelength optical data, showed that the F-region electrons had gained energy up to $\sim 60 \mathrm{eV}$ [Gustavsson et al., 2005]. This is further discussed below.

A multitude of plasma resonances are possible, that will result in electron acceleration and therefore produce optical emissions. Details of these are discussed elsewhere (see section 3), suffice to say that Kosch et al. [2002] established the fundamental importance of UHR for pumpinduced optical emissions at EISCAT. However, Ashrafi et al. [2007] showed that LT was also important at EISCAT, at least when pumping on an electron gyro-harmonic frequency (above the second) where UHR is forbidden. For pumping around the second electron gyro-harmonic at HAARP, Kosch et al. [2007c] showed that parametric decay instabilities for Langmuir, electronBernstein and upper-hybrid waves could exist as well as the thermal parametric instability (which generates striations), and that often two and sometimes three of these instabilities could co-exist simultaneously. They also found the first indirect evidence for pump-induced lower hybrid waves, which may heat the ions.

Pumping the F-region ionosphere with powerful HF radio waves produces a wide range of phenomena, many of them related to the production of optical emissions, e.g. magnetic fieldaligned plasma irregularities (see section 4.1), and stimulated electromagnetic emissions (SEE, see section 4.2). Here we address the electron temperature enhancements and pump-induced optical emissions themselves.

\subsubsection{Electron Temperature Effects}

Pump-induced optical emissions are always associated with significant increases in thermal electron temperature as observed by incoherent scatter radar. Electron temperature enhancements up to $\sim 3500 \mathrm{~K}$ using O-mode polarisation were first reported by Leyser et al. [2000] at EISCAT and are now routinely observed. Rietveld et al. [2003] showed that these maximise sharply when pumping into the magnetic zenith, consistent with the spatial distribution of the pump-induced optical emissions (see section 4.4.3). Kosch et al. [2009] reported the first, and to date only, observations of electron temperature enhancements when pumping close to the second electron 
gyro-harmonic $(2.85 \mathrm{MHz})$ using the HIPAS facility and Poker Flat incoherent scatter radar in Alaska. Although only modest electron temperature enhancements (up to $\sim 500 \mathrm{~K}$ ) were observed due to the low power of the HIPAS facility, the estimated plasma heating efficiency was approximately double that for higher pump frequencies at other facilities. Unfortunately, it seems unlikely that this interesting frequency regime can be explored further because HIPAS has ceased to exist, HAARP does not have a co-located incoherent scatter radar, and EISCAT currently cannot pump on the second electron gyro-harmonic. Gustavsson et al. [2010] successfully produced and modelled electron temperature enhancements (up to $\sim 800 \mathrm{~K}$ ) for pumping in to an under-dense ionosphere. This produces only radio wave ohmic heating and avoids plasma resonances and the production of striations. Bryers et al. [2013b] compared pump-induced electron temperature enhancements for plasma resonance (O-mode polarization) and non-resonance (X-mode polarization) plasma heating at EISCAT. For O-mode pumping, the observed height-integrated electron heating rate exceeded the ohmic electron heating rate by a factor of 2-5, the excess being attributed to the effect of plasma resonances.

Senior et al. [2012] modelled the dependence of electron temperature on the pump power at EISCAT. They found that the efficiency of the conversion of pump energy into electron thermal energy increased with power pump and approached $100 \%$ at the highest powers. This is consistent with the theoretical idea of increased conversion of electromagnetic pump wave into electrostatic upper-hybrid waves on increasingly intense striations. They also found that small changes in Dregion electron density had a significant effect on the amount of pump power reaching the F-region due to HF absorption. Bryers et al. [2013a] took the above study further to include $\mathrm{O}^{1} \mathrm{D} 630 \mathrm{~nm}$ optical emissions at EISCAT. For pump powers below the threshold $\left(\sim 37.5 \mu \mathrm{W} / \mathrm{m}^{2}\right)$ to stimulate UHR, no optical emissions were produced and the electron temperature enhancement ( 400 K) was due to ohmic heating only. For pump powers above the threshold to stimulate UHR, the electron temperature enhancement and optical emission excitation rate increased linearly with pump power. On average $\sim 70 \%$ of the pump power at the UHR altitude goes in to heating the electrons for pump fluxes above the threshold compared to $\sim 40 \%$ for fluxes below the threshold. For pump powers above the resonance threshold, $\sim 20 \%$ of the pump power was transferred to the supra-thermal electrons and $\sim 1 \%$ was converted into photons.

Bryers et al. [2013a] modelled the pump beam electric field taking D-region absorption into account to test the theoretical thresholds for the Parametric Decay Instability (PDI) and Oscillating Two-Stream Instability (OTSI), observed by the EISCAT incoherent scatter radar, and Thermal Parametric Instability (TPI) as observed by the CUTLASS SuperDARN radar. All these instabilities are thought to be associated with electron acceleration. However, TPI leads to striations and UHR, which has a clear correlation to the production of pump-induced optical emissions [Kosch et al., 2002]. Bryers et al. [2013a] found the theoretical thresholds for PDI ( 0.25 $\mathrm{V} / \mathrm{m})$, OTSI $(\sim 0.3 \mathrm{~V} / \mathrm{m})$ and TPI $(\sim 0.3 \mathrm{~V} / \mathrm{m})$ to be accurate. 


\subsubsection{Magnetic Aspect Angle Effects}

The magnetic field-aligned direction is very important for ionospheric modification experiments (see section 4.2.7). Many phenomena are favored or amplified in the magnetic zenith. This was not fully appreciated until optical observations were undertaken because of the high spatial resolution available with modern imagers.

Pump-induced optical emissions tend to be displaced from the beam towards the magnetic zenith at the EISCAT [Kosch et al., 2000], SURA [Grach et al., 2016], and HAARP [Pedersen et al., 2003] facilities. Pedersen et al. [2008] found that photon production per unit power radiated decreased by an about an order of magnitude $15-20^{\circ}$ away from magnetic zenith in any direction at HAARP. Outside this angular range, optical emissions were effectively not observed. Kosch et al. [2014a] confirmed this scenario at EISCAT, and likewise Shindin et al. [2015] at SURA, albeit for a more limited region around the magnetic zenith. Kosch et al. [2014a] also found that the optical emission always maximized in the magnetic zenith provided any pump power went in this direction. Consistent with the optical observations, the pump-induced electron temperature enhancements, observed by the EISCAT incoherent scatter radar, also maximized in the magnetic zenith [Rietveld et al., 2003].

\subsubsection{Electron Energy Spectrum}

By combining calibrated optical data at $630,557.7,844.6$ and $427.8 \mathrm{~nm}$ wavelengths with the excitation cross-sections for these emissions and EISCAT incoherent scatter radar data of electron temperature, Gustavsson et al. [2005] computed for the first time the electron energy spectrum that could explain the observations for a pump frequency close to the fourth electron gyro-harmonic. They found the accelerated electron energy spectrum extended out to $60 \mathrm{eV}$, thereby proving that the electron energy distribution had a significant non-thermal tail. A significant depression in the flux existed at $2 \mathrm{eV}$, probably caused by the electron excitation of vibrational states in molecular nitrogen that have no corresponding optical emissions. Gustavsson and Eliasson [2008] extended this work as a function of altitude to take into account the variable electron-neutral collision frequency. They found that the vibrational states of molecular nitrogen caused a reduction of the electron flux with energy between 2 and $3.5 \mathrm{eV}$ compared to thermal levels. They showed that above $\sim 3.5 \mathrm{eV}$ the electron energy distribution had a significant non-thermal tail out to $100 \mathrm{eV}$. Sergienko et al. [2012] essentially confirmed the above results at EISCAT and also showed that $>70 \%$ of the $\mathrm{O}^{1} \mathrm{D} 630 \mathrm{~nm}$ emission was produced by the thermal electrons whereas all other optical emissions required supra-thermal electrons to be present, the thermal electrons only playing a minor role in these cases.

Hysell et al. [2014] also estimated the pump-induced electron energy spectrum at HAARP for a pump frequency close to the second electron gyro-harmonic using only the 630, 557.7 and 844.6 $\mathrm{nm}$ optical emissions, and no incoherent scatter radar data was available. They found the electron energy distribution had a broad peak at $\sim 5 \mathrm{eV}$ and a long tail, which decreased exponentially by 
$30 \mathrm{~dB}$ from 5 to $50 \mathrm{eV}$. Vlasov et al. [2013] showed that the accelerated electron energy distribution depended strongly on altitude and solar activity, both of which affect the atomic oxygen $(\mathrm{O})$ and molecular nitrogen $\left(\mathrm{N}_{2}\right)$ composition of the thermosphere. It is well known that the $[\mathrm{O}] /\left[\mathrm{N}_{2}\right]$ ratio declines during high solar activity. The atomic oxygen density affects most of the observed pumpinduced optical emissions, and the molecular nitrogen density affects the flux of electrons exciting the vibrational states of nitrogen, which do not produce photons.

The results described here have been limited by the availability of multi-wavelength optical data, or the lack of an incoherent scatter radar at HAARP. Also, only a small subset of the pump frequency regime has been analyzed. Experiments where the pump frequency is stepped through the second and higher electron gyro-harmonics are required. Likewise, the $[\mathrm{O}] /\left[\mathrm{N}_{2}\right]$ density ratio needs to be known as a function of altitude at the time of observation.

\subsubsection{Small-Scale Optical Structures}

Normally, the pump-induced optical emissions form amorphous blobs within or near the pump beam (see Figures 4.11 and 4.12). However, several notable exceptions have occurred displaying sub-beam sized spatial structuring. It is only the very high resolution of modern imagers that has allowed these small-scale phenomena to be observed.

Unstable ring-like optical structures around the edge of the pump beam in the F-region, which descended in altitude and collapsed into blobs over time, could be formed at EISCAT [Kosch et al., 2004]. These were extremely sensitive to the pump beam pointing direction and were only observed for $9^{\circ}$ south of vertical. A similar phenomenon has subsequently also been observed at HAARP [Pedersen et al., 2009]. Ashrafi et al. [2007] found that the incoherent scatter radar ionline enhancements at EISCAT, which are signatures of LT, as well as the apparent generation altitude of the SEE (specifically the broad upshifted maximum) also appeared to descend in altitude simultaneously with the optical emission altitude. Only later was it realised that these descending features were signatures of ionization [Holma et al., 2006] and not the result of reduced plasma recombination due to the increased electron temperature [Ashrafi et al., 2006]. Pumpinduced ionization that produced descending layers at HAARP is discussed elsewhere in the paper (see section 4.6).

Irregular spatial structuring of the $557.7 \mathrm{~nm}$ optical emissions, of horizontal size 5-10 km, have been observed simultaneously with amorphous $630 \mathrm{~nm}$ optical emissions at HAARP [Djuth et al., 2005; Kosch et al., 2007c; Pedersen et al., 2008]. This difference is due to the much longer $\mathrm{O}^{1} \mathrm{D}$ lifetime before the photon appears compared to $\mathrm{O}^{1} \mathrm{~S}$. Kosch et al. [2007b] found that plasma depletions of order $1 \%$ within the pump beam were sufficient to focus the radio wave flux by up to $30 \mathrm{~dB}$, thereby providing a possible explanation for optical emission structures much smaller than the pump beam. Kendall et al. [2010] reported clusters of needle-like optical structures at HAARP with a horizontal size of order $100 \mathrm{~m}$ that appeared to be magnetic field-aligned and filled

much of the pump beam. These very small-scale optical structures appeared to move over time 
and probably also descended in altitude. Bernhardt et al. [2016] reported that such small-scale optical structures could be maintained for long periods (hours) as long as the pump beam remained turned on. They appeared to drift across the image, probably with the background thermosphere wind.

Small-scale E-region optical emissions of order $1 \mathrm{~km}$ in size, named "speckles", have been observed in $557.7 \mathrm{~nm}$ at HAARP [Pedersen and Gerken, 2005; Pedersen et al., 2009]. E-region pump-induced optical emissions have never been observed at EISCAT.

\subsubsection{X-Mode Optical Phenomena}

Plasma resonances are usually stimulated by using O-mode polarization because this pump wave reflects above the UHR resonance altitude, which is important for producing optical emissions [Kosch et al., 2002]. In addition, the O-mode pump wave has a large field-parallel electric field component close the HF reflection altitude, which favours LT.

Gustavsson et al. [2009] first noticed that optical emissions produced by O-mode pumping of the ionosphere were significantly reduced in intensity by simultaneous X-mode pumping. The Xmode frequency was set $700 \mathrm{kHz}$ higher than the O-mode frequency to ensure a similar $\mathrm{HF}$ reflection altitude. The mechanism is thought to be X-mode suppression of the growth of O-mode generated striations, which are symbiotic with the UHR required to accelerate the electrons. Xmode ohmic heating of the electrons raises their temperature, which increases the threshold for the thermal parametric instability required to generate striations.

Conventional wisdom dictates that $\mathrm{X}$-mode polarized waves cannot produce the UHR because the pump wave reflects below the UHR altitude. Unlike O-mode polarization, the X-mode waves do not have an electric field component parallel to the magnetic field line direction near HF reflection, and are therefore should not be able to produce LT either. However, these theoretical notions have proven to be incorrect, at least under certain circumstances. Blagoveshchenskaya et al. [2011] found X-mode pump waves at EISCAT could produce field-aligned striations. Blagoveshchenskaya et al. [2013] found that by pumping slightly above the O-mode critical frequency and using high pump powers, strong electron temperature enhancements (up to 50\%) and the production of striations was possible. Underdense pumping presumably allowed the Xmode wave to reach the UHR altitude because there is no HF reflection. Specifically, they found that $f_{H}-f_{c e} / 2 \leq f x F 2 \leq f_{H}+f_{c e} / 2$ was necessary, where $\mathrm{f}_{\mathrm{H}}$ is the pump frequency, $\mathrm{f}_{\mathrm{ce}}$ is the electron cyclotron frequency, and fxF2 is the X-mode critical frequency. Blagoveshchenskaya et al. [2015] found that signatures of LT (EISCAT incoherent scatter radar ion and plasma line enhancements) during X-mode pumping for $f_{O} F 2 \leq f_{H} \leq f x F 2$, where $f_{O} F 2$ is the O-mode critical frequency. Narrowband SEE could also be generated.

Blagoveshchenskaya et al. [2014] showed that intense $\mathrm{O}^{1} \mathrm{D}(630 \mathrm{~nm}$ at $1000 \mathrm{R})$ and $\mathrm{O}^{1} \mathrm{~S}(557.7$ $\mathrm{nm}$ at $250 \mathrm{R}$ ) optical emissions were produced by X-mode pumping (see Figure 4.13). These emissions were more intense than any O-mode optical emission ever recorded at EISCAT, 
providing evidence that $\mathrm{O}$-mode leakage from imperfect forming of the X-mode pump beam could not have been the cause of the X-mode phenomena observed. Blagoveshchenskaya et al. [2017] found that $\mathrm{X}$-mode phenomena were pump frequency dependent relative to an electron gyroharmonic, which is similar to the more familiar O-mode observations.
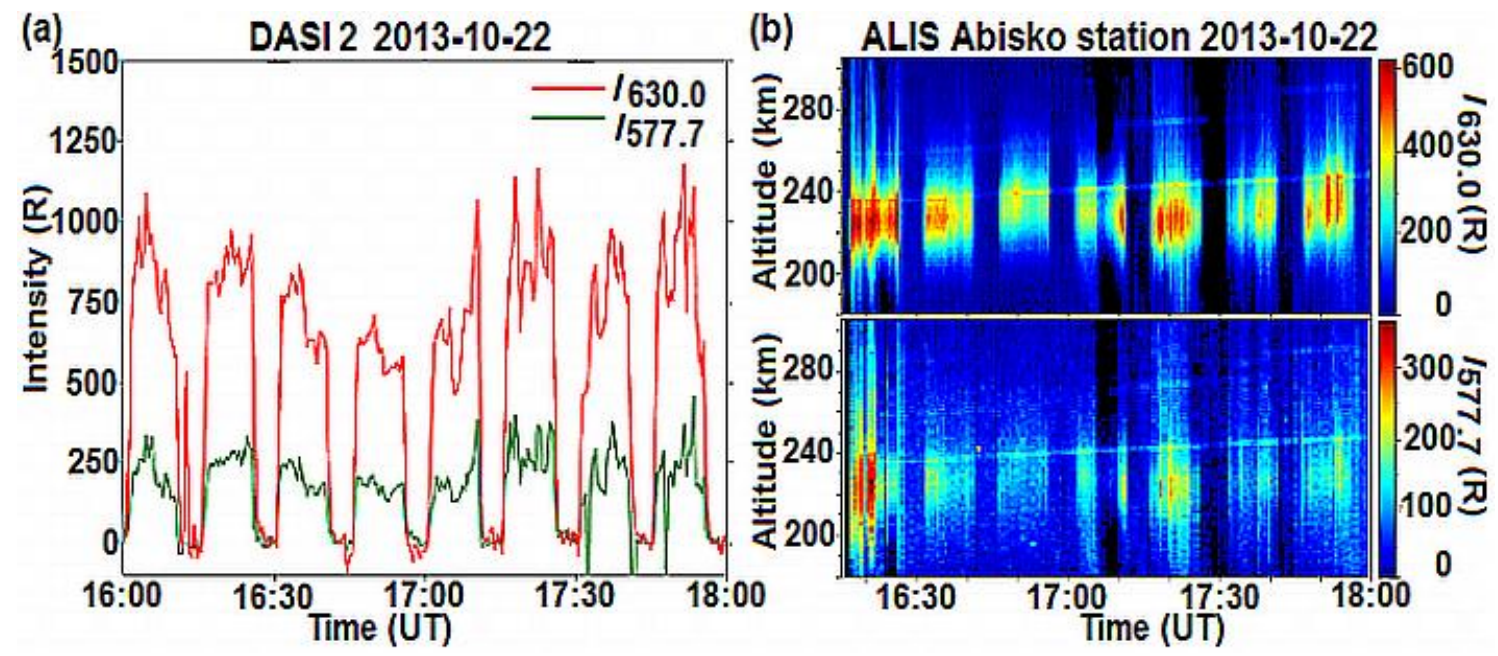

Figure 4.13. X-mode pump-induced optical emissions at EISCAT from 22 October 2013 using a 10-min on, 5-min off pump cycle [Blagoveshchenskaya et al., 2014]. (a) $\mathrm{O}\left({ }^{1} \mathrm{D}\right)$ and $\mathrm{O}\left({ }^{1} \mathrm{~S}\right)$ optical emissions observed (a) field-aligned from the EISCAT site, and (b) obliquely from Abisko, Sweden, about $140 \mathrm{~km}$ south of EISCAT.

\subsubsection{Optical Phenomena in the $\mathbf{E}$ Region}

It is difficult to generate optical emissions in the E-region because the plasma density is normally too low to allow plasma resonance with HF pump waves. In the cases where particle precipitation raises the plasma density sufficiently, the accompanying auroral emissions dominate any pump-induced optical emissions.

Kagan et al. [2000] first observed the pump-induced $\mathrm{O}^{1} \mathrm{~S} 557.7 \mathrm{~nm}$ optical emission from a sporadic-E layer over the low-latitude Arecibo facility. They proposed that the optical emissions could be used to image the irregular structures within sporadic-E layers because holes in the layer would allow the pump wave to pass through. Any optical emissions generated in the F-layer would be dominated by the $\mathrm{O}^{1} \mathrm{D} 630 \mathrm{~nm}$ optical emission. Similar structured E-region pump-induced $\mathrm{O}^{1} \mathrm{~S}$ $557.7 \mathrm{~nm}$ optical emissions were reported from the mid-latitude SURA facility [Bakhmet'eva et al., 2005].

Pedersen and Gerken [2005] reported the first naked-eye visible pump-induced $\mathrm{O}^{1} \mathrm{~S} 557.7 \mathrm{~nm}$ optical emissions from the HAARP facility. These small-scale "speckles" occurred on the background of naturally-occurring pulsating auroras at about $110 \mathrm{~km}$ altitude. Pedersen et al. [2009] made further similar observations of the speckles from HAARP, with an estimated size of order $1 \mathrm{~km}$. The spatial structuring mechanism remains unexplained. 
Perhaps somewhat surprising, no E-region pump-induced optical emissions have been reported from the EISCAT facility.

Kagan et al. [2006] made the first and only observation of pump-induced OH(9-3) Meinel optical emissions at $629.79 \mathrm{~nm}$, which originates from 80-85 km altitude, from the SURA facility. Although the filter also passes the $\mathrm{O}^{1} \mathrm{D} 630 \mathrm{~nm}$ emission as well as, the rapid rise time of the optical emission indicates that it is not from this source.

\subsubsection{Other phenomena}

Pump-induced optical observations offer a number of possible applications. Sergienko et al. [1997] reported that ionospheric pumping with a $0.5 \mathrm{~Hz}$ modulation at EISCAT could modify a naturally occurring morning diffuse aurora observed by a TV camera. The modulation was very small and could only be detected indirectly by spectral analysis. The phenomenon was explained by the decrease in dissociative recombination when the electron temperature is enhanced. Blagoveshchenskaya et al. [2001] reported triggering of local auroral activations (an auroral arc modification and its subsequent break-up) by ionospheric pumping into a sporadic E layer at EISCAT. Such experiments are difficult to repeat and may require special geophysical conditions. Ruzhin et al. [2012] provided evidence that triggering a substorm by ionospheric pumping at the SURA facility might sometimes be possible if the magnetosphere was already primed.

The $\mathrm{O}^{1} \mathrm{D}$ atom has a long radiative lifetime in vacuum, which is sensitive to collisional relaxation. Reduced decay times of the $630 \mathrm{~nm}$ emission can be attributed to collisions with atmospheric species. Kalogerakis et al. [2009] demonstrated that atomic oxygen density between 200 and $300 \mathrm{~km}$ altitude could be obtained by observing the decay rate of pump-induced $630 \mathrm{~nm}$ emissions.

Kosch et al. [2014b] combined EISCAT incoherent scatter radar observations of pumpenhanced electron temperature as well as ion temperature and velocity, and electron density with the MSIS model of neutral density, to infer the field-aligned anomalous electric field in the topside ionosphere $(390-580 \mathrm{~km})$. As expected, this was in the $\mu \mathrm{V}$ range pointing downwards. By including calibrated observations of the simultaneous pump-induced optical emissions, they also estimated the field-aligned anomalous resistivity.

\subsection{ULF/ELF/VLF Waves}

\subsubsection{Generation of ULF/ELF/VLF Waves}

There are several different mechanisms/techniques for the generation ULF, ELF and VLF waves propagating into the magnetosphere and into the earth-ionosphere waveguide with the HF heating. The first and the most popular mechanism is a temporal modulation of the ionospheric conductivity in the $\mathrm{D}$ and $\mathrm{E}$ ionospheric regions when the electric field exists in the ionosphere. This approach had been proposed by Getmantsev et al. [1974] and the basic physics of it is that the heating of electrons in the lower ionosphere causes two effects: First, it changes the electron 
collision frequency and second it reduces the electron-ion recombination rate. Both effects lead to a generation of localized disturbances in plasma density (and the ionospheric Pedersen conductivity), which generate magnetic field-aligned currents (FACs) when the electric field exists in the ionosphere.

If these FACs are modulated in the ULF/ELF frequency range, then they will propagate into the magnetosphere in the form of electromagntic waves and experiments involving heating of the ionosphere with $\mathrm{HF}$ transmitters already prove that this mechanism can generate noticeable waves in that frequency range detected in the magnetosphere [Stubbe et al., 1981; Robinson et al., 2000; Cohen et al., 2011; Cohen and Inan, 2012]. Combined operations of the HAARP transmitter with the DEMETER satellite had been used to study in more detail this mechanism using different modulation schemes.

In the experiment reported by Piddyachiy et al. [2008] the HAARP transmitter antenna was directed upwards with an effective radiated power of $407 \mathrm{MW}$ in the center of the beam at a frequency of $3.25 \mathrm{MHz}$ to maximize the heating of the D and lower E region. Several combined operations with the HF pump-wave modulation from $\sim 500 \mathrm{~Hz}$ to $\sim 4.5 \mathrm{kHz}$ were performed on February 26, 2007 during night time passes of DEMETER over HAARP at 21.30 LT. Electric and magnetic signals were detected by DEMETER antennas and the observations, illustrated in

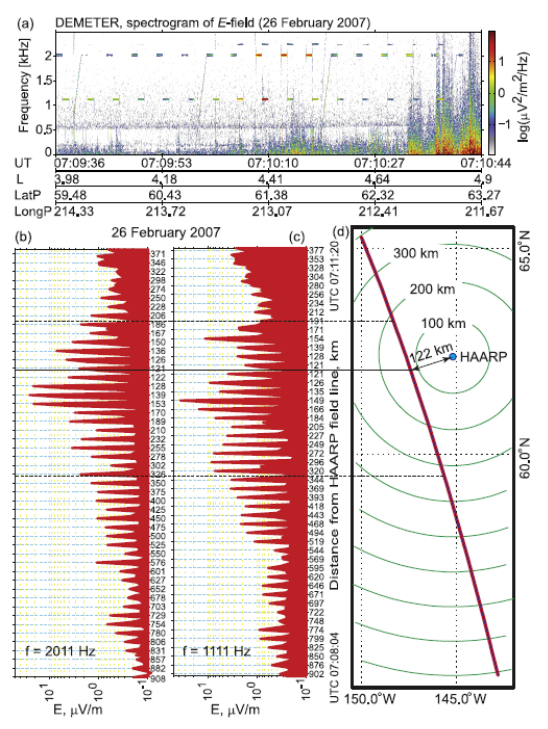

Figure 4.14. Top panel: Spectrogram of the VLF electric field component E12 ( Ey). Bottom left panels: $1 \mathrm{~s}$ average of the intensity of E12 at 2011 and $1111 \mathrm{~Hz}$ within a narrow frequency band of $4.9 \mathrm{~Hz}$. Bottom right pannel: Projection along the Earth's magnetic field of the satellite position down to $75 \mathrm{~km}$, the altitude of the source. [Piddiachyi et al., 2008].
Figure 4.14 for orbit 14157_1 on February 26, 2007, may be summarized as follows. ELF/VLF waves are detected along magnetic field lines that intersect the $D$ region at $\sim 75$ $\mathrm{km}$ altitude over three main regions: from $\sim 200 / 300$ to $\sim 900 \mathrm{~km}$ from the vertical of HAARP intermittently and with an average weak intensity, at distances less than $\sim 200 / 300 \mathrm{~km}$ with a higher intensity and more frequently and finally at distances less than $\sim 100 / 150 \mathrm{~km}$ and in narrow channels of $\sim 10-20 \mathrm{~km}$ extent with a very large intensity.

Combining results from a full wave numerical model [Lehtinen and Inan, 2008] and DEMETER observations, Piddyachiy et al. [2008] have concluded that in the first region waves originally injected in the Earth-Ionosphere waveguide may leak along magnetic field lines and propagate in the whistler mode to the satellite. In the second region, direct injection along magnetic field lines of waves propagating at rather large oblique incidence from the modulated electrojet may also occur, leading to sporadic enhanced signals. The very intense waves 
observed over 10-20 km along the satellite path at less than 100-150 km from HAARP correspond to waves propagating close to vertical and directly injected in the upper ionosphere.

Two other heating techniques, closely related to the first one, are 1) a so-called "beam painting" and 2) geometric modulation. The beam painting technique means that the beam focusses in a small spot and this spot is moving rapidly across some area in the ionosphere to heat electrons inside this area. The whole process is modulated with the ULF/ELF frequency.

Geometric modulation means that instead of heating one spot (or some area) in the ionosphere and turning the transmitter $\mathrm{ON}$ and $\mathrm{OFF}$ with different periodicities, the transmitter sends a constant beam of HF power and move it in the ionosphere along some particular path. This type of heating requires a phased array transmitter because the beam should change its orientation relatively rapidly.

Figure 4.15 adapted from Cohen et al. [2010] illustrates the difference between amplitude modulation, beam painting and geometric modulation techniques used in experiments at HAARP and the results from a number of experiments are discussed in that paper. Experiments at EISCAT measured the heating and cooling time constants in the lower ionosphere in order to evaluate theoretical aspects of "beam painting" but concluded that the fundamental and odd harmonics will not be greatly enhanced but the even harmonics can be [Barr et al., 1999]. This is because the even harmonics are sourced from a lower height where the heating and cooling times differ significantly, compared to the fundamental and odd harmonics.

DEMETER's observations and modeling results indicate that wave injection in the magnetosphere is achieved more efficiently, by 5 to $7 \mathrm{~dB}$, using the steered modulation technique than the classical time modulation technique. Further ground observations showed that geometric modulation is less efficient than time modulation below $\sim 2 \mathrm{kHz}$ but significantly more efficient by as much as 7 to $11 \mathrm{~dB}$ above $\sim 3 \mathrm{kHz}$ in particular for long distances.

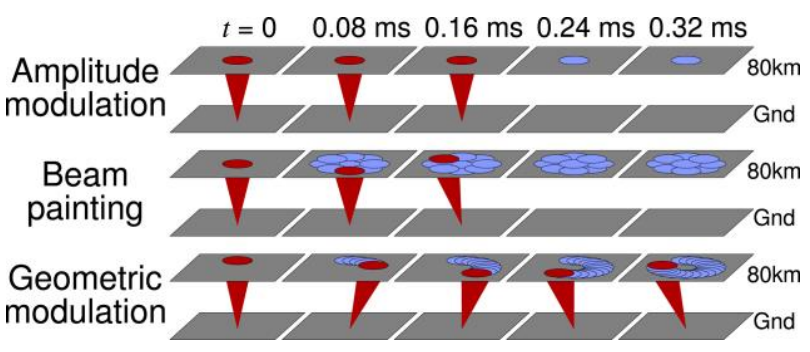

Figure 4.15. Schematic comparison of amplitude modulation ( $\mathrm{AM})$, beam painting $(\mathrm{BP})$, and geometric modulation $(\mathrm{GM})$. In cases of $\mathrm{AM}$ and $\mathrm{BP}$ the beam is turned ON and OFF during the half of the ELF/VLF wave period (which is $4 \mathrm{~ms}$ for $f=2.5 \mathrm{kHz}$ ). In GM case the constant beam (no temporal modulation) makes a slower

More "exotic" techniques of wave generation with the ionosphereic heating sweep along a geometric shape, in this case a circle [from Cohen et al., 2010].

include:

1. The Ionospheric Current Drive (ICD) mechanism proposed by Papadopous et al. [2011a, 2011b] for modulation frequencies in the range 1-20 Hz. The idea behind ICD is that heating of the ionosphere with O-mode waves increases electron temperature near the F2 peak and creates preasure disturbance there (see Figure 4.16). According to the MHD theory the presure perturbation causes disturbance of the magnetic field, which causes the Hall current and the Hall 
current can couple to the Pedersen current in the E region. The main advantage of this mechanism is that it does not require electric field in the E region [Papadopoulos et al., 2011a; 2011b; Eliasson et al., 2012].

2. Rotating Magnetic Field (RMF) mechanism based on producing magnetic field with different polarization by rotating superconducting or permanent magnets [Gigliotti et al., 2009; Karavaev et al., 2010; 2011]. There have not been any space experiments based on this technique yet, but experiments conducted in the laboratory plasma (in particular, on the LAPD machine at UCLA) and the corresponding three-dimensional MHD simulations demonstrated that the rotating magnetic field antenna composed of two perpendicular coils with alternating currents set at $90^{\circ}$ out of phase can efficiently generate ULF and VLF waves with the polarization depending on that of the antenna.

3. "Pre-heating" of the ionosphere with a long heating pulse, followed by the modulation at the desired ELF/VLF frequency. The idea here is that the long pulse reduces the electron-ion recombination coefficient, resulting in increased ambient electron density and current density. It was theoretically shown by Milikh and Papadopoulos [2007] that such two-timescale heating can increase significantly (up to $7 \mathrm{~dB}$ ) the efficiency of the heating and produce VLF signals with larger amplitudes.

4. Beat-wave ELF/VLF generation [Barr and Stubbe, 1997, Kuo et al., 2011; 2012; Cohen et al., 2012b; Moore et al., 2012]. In this approach two continuous HF signals with a frequency difference in the ELF/VLF range are transmitted. The power of the transmitted waves oscillates at the beat frequency and modulates the electron temperature in the lower ionosphere, produces density/conductivity inhomogeneities and generates EM waves, if an electric field exists in the ionosphere. The beat-wave generation may use two spatially separated HF sourses (or heated spots in the ionosphere) and introduce some geometrical factor in the heating experiment. Beat wave

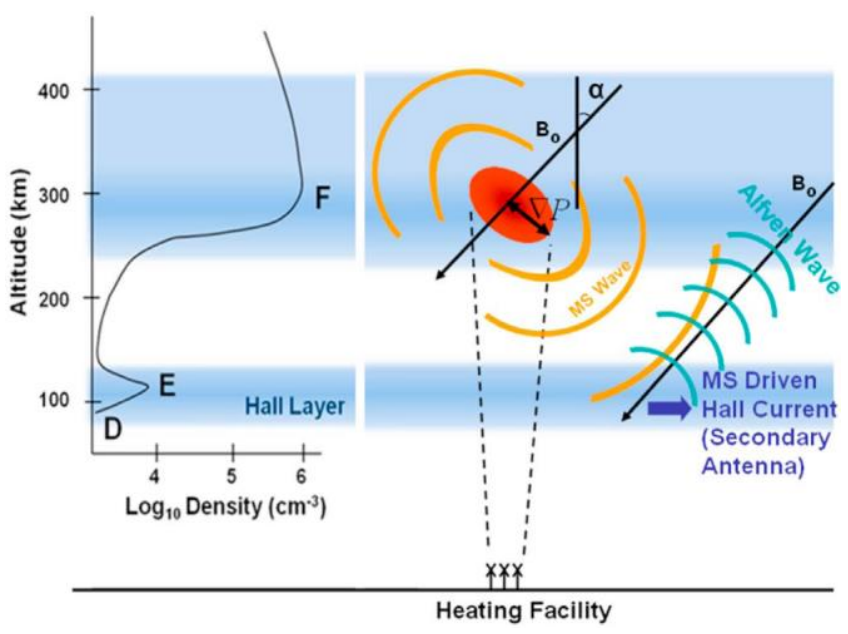

Figure 4.16. Schematic of the Ionospheric Current Drive (ICD) concept. Periodic heating of $\mathrm{F}$ region leads to a diamagnetic current and an oscillatory field aligned magnetic moment that radiates isotropic magnetosonic (MS) waves. The E-field of the MS wave drives Hall currents in the $\mathrm{E}$ region, resulting in a virtual antenna that injects waves in the earth-ionosphere waveguide and shear Alfvén waves in the magnetosphere [from Papadopoulos et al., 2011]. generation may be stronger than amplitude modulation depending on various parameters like antenna spacing, ELF/VLF frequency, and direction of the receiver.

5. Excitation of ELF and VLF waves using the cubic thermal nonlinearity, which involves interactions between the electric fields and the polarization current associated with two high-power HF waves with frequencies $f_{1}$ and $f_{2}$, where $f_{2} \approx 2 f_{1}$ [Barr, 1996; Kotik and Ermakova, 1997]. It is 
expected that $f_{1}$ wave induces a collision frequency oscillation at $2 f_{1}$ frequency and that these oscillations of the collision frequency will interact with the oscillations of the polarization current density caused by $f_{2}$ wave and produce ELF and VLF source current density with the frequency $\mid$ $f_{2}-2 f_{1} \mid$. Moore et al. [2013] demonstrate that the cubic generation of ELF and VLF waves is substantially weaker than the electrojet modulation in the $1-5 \mathrm{kHz}$ range. Signals produced by this mechanism are also weaker than the signals reported to be generated by the ICD mechanism at frequencies $<100 \mathrm{~Hz}$, but they can be stronger at frequencies $>10 \mathrm{kHz}$.

6. A new mechanism generating EM VLF waves in frequency ranges $7-10 \mathrm{kHz}$ and $15-19$ $\mathrm{kHz}$ with constant HF heating was recently proposed by Vartanyan et al. [2016]. It does not rely on any VLF modulation of HF emissions and was observed in absence of any electrojet. The corresponding observations were performed during two daytime HAARP/BRIOCHE sessions during flyovers of the DEMETER satellite and we briefly summarize in the following the results and interpretation of the first session.

This experiment was conducted during DEMETER orbit 28313_0 on October 16, 2009 in a quiet ionosphere with $f_{O} F 2=5.15 \mathrm{MHz}$. HAARP was set to operate in $\mathrm{CW}$ mode, emitting in the O-mode at its maximum power of 3.6 MW and with the HF beam directed along the magnetic zenith. The frequency of the pump-wave was $f_{H}=5.1 \mathrm{MHz}$, corresponding to a reflection altitude of $\sim 220 \mathrm{~km}$. The closest distance of DEMETER from the magnetic field line along which HAARP HF waves were injected was $69 \mathrm{~km}$. In addition to DEMETER measurements, ground based diagnostics included (i) a magnetometer, indicating very weak disturbances thus no significant electrojet, (ii) stimulated electromagnetic emission (SEE) observations and (iii) Slant TEC (STEC) measurements. Displayed in Figure 4.17 are 1 minute (20:32:15-20:33:15) of DEMETER data around closest approach: ELF $(20 \mathrm{~Hz}-2 \mathrm{kHz})$ and VLF $(5-20 \mathrm{kHz})$ spectra of the electric component E12 perpendicular to the orbit plane and the $\operatorname{VLF}(5-20 \mathrm{kHz})$ spectrum of the magnetic component at $45^{\circ}$ from orbit plane. The ELF turbulence spectrum of the electric component indicates that DEMETER crosses a first narrow heated flux tube extending $\sim 15 \mathrm{~km}$ along the orbit and a few seconds later the main heated flux tube extending $\sim 140 \mathrm{~km}$ along the orbit. Within the heated flux tubes a strong signal between $\sim 7.5 \mathrm{kHz}$ and $\sim 8.5 \mathrm{kHz}$ develops and also faint emissions can be noticed between $\sim 15.8$ and $16.9 \mathrm{kHz}$ thus at harmonic frequencies of the main one.

The EMI background noise on magnetic measurements, in particular the numerous parasitic lines in the 7.5-8.5 kHz frequency range of interest, makes it impossible to use the magnetic data for a thorough analysis of the wave observations. The strong signals observed on the electric component in the heated flux tube are in the frequency range typical of the lower hybrid frequency in the $F$ region at HAARP latitude. 
However, Vartanyan et al. [2016] argue that electrostatic waves that are known to exist in the heated plasma close to the altitude of reflection of the pump-wave cannot propagate to large distances and reach DEMETER at $650 \mathrm{~km}$ altitude. In addition the only electrostatic waves which could be thought of are the lower hybrid (LH) waves with a frequency around $8 \mathrm{kHz}$ as is observed but no known mechanism can produce simultaneously ES waves at the second harmonic of this frequency as detected by DEMETER. The authors conclude that the only process consistent with the observations is the linear and non-linear conversion in the heated region of LH waves to whistler waves which propagate upwards along the field lines to be ultimately detected by DEMETER. The detailed plasma wave processes are quite complex and call first for the parametric excitation of LH waves by the interaction of the HF pump-wave with the ionospheric plasma at the upper hybrid (UH). The existence of these waves was inferred from the SEE spectrum recorded on ground showing a down-shifted maximum at $\sim 8 \mathrm{kHz}$. The next step is the interaction of these LH waves with meter-scale field-aligned striations to generate whistlers at the LH frequency.

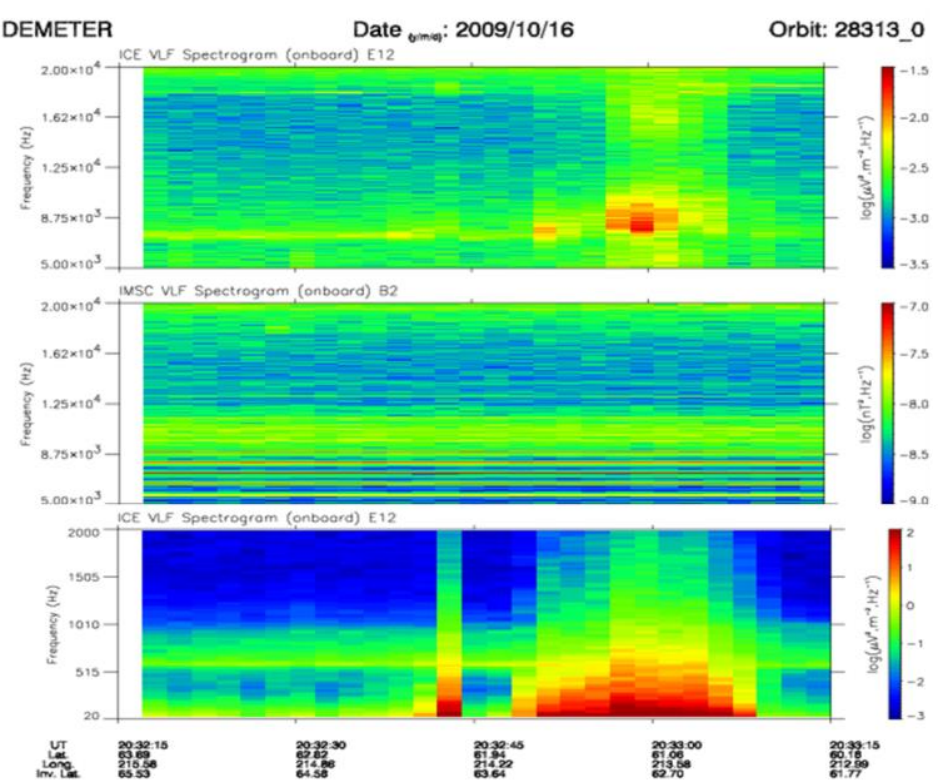

Figure 4.17. Upper pannel: Spectrogram of the VLF Magnetic field component at $45^{\circ}$ from E12 5-20 kHz. Middle panel: Spectrogram of the VLF E12 Electric Field component 5-20 kHz. Bottom panel: Spectrogram of the ELF E12 Electric Field component $0-2 \mathrm{kHz}$. From Vartanyan et al. [2016].
Again, the increase of STEC revealed by ground-based measurements provide the indication that plasma density striations are developing [Milikh et al., 2008b]. As far as the second harmonic whistler waves are concerned, interaction of counterpropagating $\mathrm{LH}$ waves is proposed by the author as a likely mechanism. This model was supported by numerical simulations and is in good agreement with the observations.

The important difference between these types of heating is that some of them are conducted with $\mathrm{X}$-mode and others with $\mathrm{O}$-mode HF waves. Ohmic absorption is stronger for X-mode than O-mode and stronger for lower frequencies. In addition there is the possibility that X-mode waves interact with the electrons via cyclotron resonance and the heating is most efficient when the frequency of the pump wave is close to the electron gyrofrequencies in the ionospheric D/E region and the beam of HF power from the transmitter is pointed in the direction of the local magnetic zenith (along the geomagnetic field).

Results from numerous experimental studies of heating efficiency for generation of ULF/ELF 
waves suggets that the most efficiency (at least at HAARP) is achived when the heating is conducted with X-mode waves with frequency $2.75 \mathrm{MHz}$ or $3.25 \mathrm{MHz}$. These are the lowest HF frequencies available with HAARP.

At the same time, heating with higher frequencies gave an increase in the effective radiated power (ERP) and allow to focus the pump power in a smaller spot in the ionosphere, which again, increases the heating efficciency. For example, the size of the heated spot produced by HAARP at the altitude of $100 \mathrm{~km}$ for the $3 \mathrm{~dB}$ beam width of $4.57 \mathrm{MHz}$ vertical beam is $\approx 20 \mathrm{~km}$. The total power of the HAARP transmitter is 3.6 MW and its ERP depends on frequency and changes from $427 \mathrm{MW}$ for $2.75 \mathrm{MHz}$ wave to $1023 \mathrm{MW}$ for $4.57 \mathrm{MHz}$ wave [Streltsov et al., 2014].

\subsubsection{Resonant ULF Waves}

In contrast to ELF/VLF wave generation with frequencies greater than about $1 \mathrm{~Hz}$, where electron collision frequency or temperature in the lower $\mathrm{D}$ region is the modified parameter causing the current to generate the waves, ULF waves from 1 second to hundreds of seconds period can also be produced by electron density changes in the upper $\mathrm{D}$ and $\mathrm{E}$ regions. The time constant for electron temperature-dependent ion recombination rate to change is rather long so that this effect is only important for periods greater than about several seconds. Such long period ULF waves have been much more difficult to reliably generate at EISCAT and indeed some of the early results of Pc4-Pc5 waves generated by Heating have been questioned. All reported cases of ULF wave detection have been made under or close to the heated ionosphere, so that the magnetic field of the locally perturbed current system was measured. The results from these early Pc4-Pc5 excitation experiments are summarized in [Stubbe,1996] but there have been few reports since the early 1980's. There have been no cases of ULF waves being observed after the heating was switched off, which would have been an indication of Alfvén wave propagation to the opposite hemisphere and back.

An important measurement in support of ULF current modulation in the E region would be the simultaneous measurement of electron temperature and density changes by an incoherent scatter radar while ULF pulsations are measured by a magnetometer. Such measurements of E region perturbations have been very rare and they have not been convincingly correlated with magnetic field measurements. E-region plasma instabilities have been excited through resonance instabilities [Hibberd et al., 1983; Hoeg et al., 1986; Hysell et al., 2010] and it might be expected that the energy dissipated by them would contribute to electron heating, electron density modulation and current modulation. This is still an area worth investigating in the future, in particular with a more powerful radar like EISCAT_3D which could measure these parameters as well as the electric fields within and outside of the heated region practically simultaneously.

Numerical and experimental studies demonstrate that the generation of ULF waves can be

much more efficient if the driver is modulated in time with the frequency of the wave standing inside some resonator cavity. There are two such resonators in the magnetosphere schematically 
shown in Figure 4.18. The first one is called a global magnetospheric resonator or field line resonator (FLR) and is formed by the entire closed magnetic flux tube bounded by the ionosphere. The second resonator, called the ionospheric Alfvén resonator (IAR), is formed by the conducting bottom of the ionosphere and a strong gradient in the Alfvén speed at the altitude $\approx 0.5-1.0 R_{E}$ above the ground [Polyakov and Rapoport, 1981].

The eigenfrequencies of the resonat waves are defined by the size of the resonator, distribution of the Alfvén speed inside, and the boundary conditions on the walls of the resonator. Because the sizes of these two cavities are quite different, the eigenfrequensies of FLR and IAR are
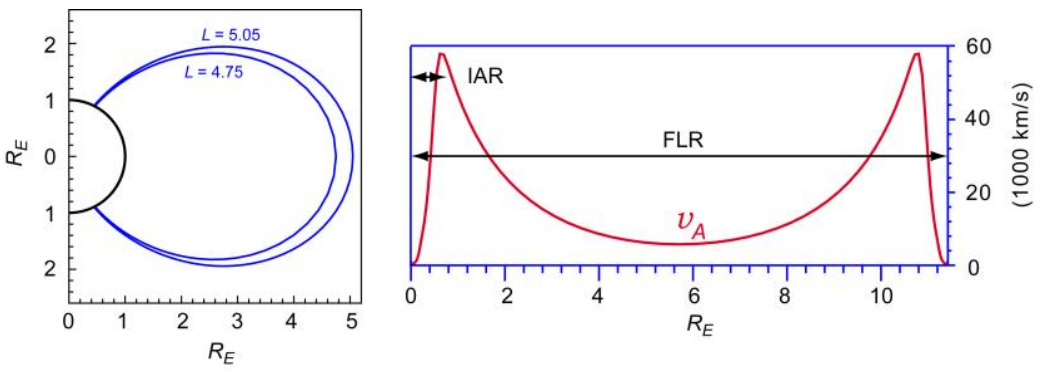

Figure 4.18. (Left) Dipole magnetic flux tube corresponding to HAARP's magnetic latitude. (Right) Schematic plot of two magnetospheric resonators: FLR and IAR.

different as well. The typical eigenfrequency of IAR is in the range 0.1-10 Hz [Polyakov and Rapoport, 1981] and the typical eigenfrequency of FLR is in the range 0.9-10 $\mathrm{mHz}$ [Samson et al., 1992].

ULF waves can be generated in these resonators by several different physical mechanisms including wave-wave and wave-particle interactions. One of the currently most acceptable natural drivers for these waves is the ionospheric feedback instability (IFI), introduced by Atkinson [1970]. The basic idea of this instability is that the field-aligned current in an ULF Alfvén wave changes the ionospheric density and conductivity by precipitating/removing electrons into/from the E layer, and these variations in the conductivity "feed back" on the structure and amplitude of the incident wave. When the ionospheric feedback works in a constructive way, the conditions for IFI are satisfied and the amplitude of the wave inside the resonator and density disturbances on the ionospheric boundary increase.

IFI has been extensively studied at middle and high latitudes and all these studies agreed that the favorable conditions for the instability include low state of the ionospheric density/conductivity and the presence of a relatively large $(>20 \mathrm{mV} / \mathrm{m})$ electric field in the ionosphere. One more favorable condition for the IFI

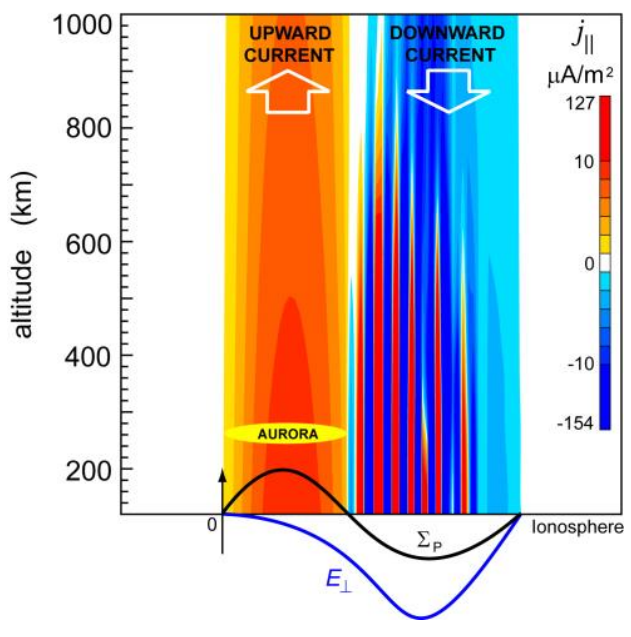

Figure 4.19. Schematic plot of interactions between upward and downward magnetic field-aligned currents and the ionosphere [Streltsov et al., 2010a]. development is matching between the ionospheric and magnetospheric "impedances", namely, $\Sigma_{P}$ $\approx \Sigma_{A}$. Here, $\Sigma_{\mathrm{P}}$ is the height-integrated Pedersen conductivity of the ionospheric E region and $\Sigma_{A}=$ 
$1 / \mu_{0} v_{A}$ is the wave conductance in the low magnetosphere ( $v_{A}$ is the Alfvén speed above the $\mathrm{E}$ region). The large-scale electric field in the ionosphere serves as an energy source for the instability, and the "matching impedance" condition provides a strong electromagnetic coupling between the ionosphere and the magnetosphere [Trakhtengertz and Feldstein, 1984].

The strong electric field in the ionosphere exists in the polar electrojet region and the ionospheric conductivity is low during the nighttime and winter season. These conditrions are also satisfied when and where the ionosphere interacts with a large-scale downward magnetic fieldaligned current. From the current continuity condition one can expect that such current will be adjacent to the upward current channel, responsible, for example, for a discrete auroral arc. Figure 4.19 from Streltsov et al. [2010a] shows a schematic plot of interactions between two large-scale magnetic field-aligned currents and the high-latitude ionosphere. This figure shows that the smallscale $(\approx 10 \mathrm{~km})$, intense FACs carried by ULF shear Alfvén waves are generated by IFI inside a large-scale downward FAC channel.

One of the main questions is how IFI starts. It is usually assumed that the instability is "seeded" by a small-scale density disturbance in the E region. If its size matches the transverse wavelength of the most feedback-unstable mode, defined by the ionospheric and magnetospheric parameters, then the instability develops quite rapidly. This fact suggests that IFI can be triggered artificially with a ground-based HF transmitter and numerical simulations by Streltsov et al. [2005] demonstrate that HF heating may not only trigger IFI inside the FLR but can also enhance its development if the HF power is modulated with the period of the most feedback-unstable mode. These results were used as a motivation for the experiment conducted at HAARP on 29 October 2008. The goal of the experiment was to trigger and amplify IFI in the downward current region adjacent to a bright, discrete auroral arc.

There have been claims of artificial $\mathrm{E}$ region perturbations at EISCAT triggering a substorm [e.g., Blagoveshchenskaya et al., 2001] but it is hard to prove that a single event like this one was not a coincidence and convincing measurements of $E$ region perturbations to electron density and temperature were lacking. One of the important measurements still to be made is of the changes to the conductivity, currents and electric fields in and around the HF-modified E region, a task which the projected EISCAT_3D radar [McCrea et al., 2015] with its multiple, fast scanning beams and volumetric measurements may be able to achieve.

\subsubsection{ULF Waves in the Global Magnetospheric Resonator}

To excite ULF waves in the global magnetospheric resonator the eigenfrequencies of the coupled magnetosphere-ionosphere system must be identified in advance. These eigenfrequencies can be obtained from simulations of the MHD equations if parameters of the ionosphere and the magnetosphere are known. Magnetospheric parameters (magnetic field, plasma density and temperature) can be obtained from the models derived from first principles or direct satellite observations. Parameters of the ionosphere, in particular the density inside the $\mathrm{E}$ and $\mathrm{F}$ regions, 
can be estimated from the international reference ionosphere (IRI) model or from the observations made by the HAARP digisonde or an ISR during the same time of the day one year before the experiment.

Unfortunately, an ionosond or ISR shows that magnitudes of plasma density and the electric field in the ionosphere may change quite rapidly within broad limits, particularly during the geomagnetically active time. Therefore, before the experiment begins, it is necessesary to calculate a large number of possible eigenfrequencises associated with IFI for different combinations of geophysical parameters. For example, in preparation for the October 292008 experiment at HAARP, 30 eigenfrequencies had been calculated for different possible combinations of the ionospheric parameters. During the experiment HAARP transmitted 4.2 MHz X-mode waves in the direction of the magnetic zenith. The frequency of modulation of these waves had been choosen from these 30 eigenfrequencies based on the data provided by the HAARP digisonde during the experiment.

Figure 4.20, reproduced from [Streltsov et al., 2010a], shows variations of three components of the magnetic field measured by the fluxgate magnetometer at Gakona (the closest site to HAARP). It shows that the experiment began during quiet geomagnetic conditions and a magnetic disturbance with a magnitude greater than $250 \mathrm{nT}$ occurred within $\sim 40 \mathrm{~min}$ of heating. Note that the schedule for the experiment had been finalized a month before the experiment begun.

During the experiment ground-based magnetometers in Alaska and Canada detected large-amplitude ULF waves in regions where the substorm onset auroral arcs interacted with the ionosphere. The frequencies of these waves closely matched frequencies predicted by the simulations of IFI for these particular geophysical conditions (see Figure 4.21). Therefore, observations conducted during the 29 October 2008 HAARP experiment strongly support the hypothesis that geomagnetic substorms and the corresponding dynamics of discrete auroral arcs

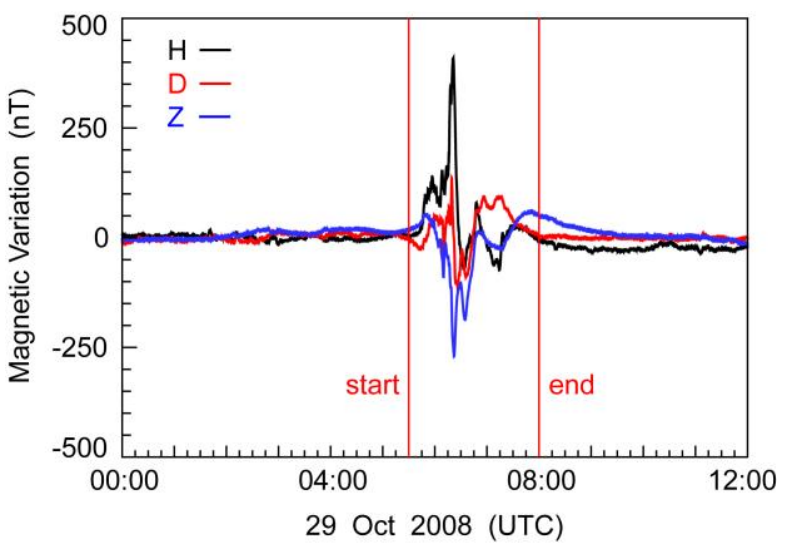

Figure 4.20. Three components of the magnetic field measured by the fluxgate magnetometer at Gakona, Alaska during the October 29, 2008 experiment at HAARP. Red vertical lines mark the start and the end of the experiment [Streltsov et al., 2010a]. are closely connected with the development of IFI and generation of large-amplitude ULF waves. The main argument in favor of this idea is that for the first time frequencies of the waves generated by IFI in the downward current channel adjacent to the bright auroral arc had been predicted from the simulations of IFI before the experiment began.

Another technique for the excitation of IFI with heating was proposed by Streltsov and Pedersen [2010]. It is based on the fact that IFI produces ULF waves propagating across the 
magnetic field in the direction of the background electric field and reaching maximum amplitude not at the location where the instability started (or where the heating initiates the instability), but further down in the direction of the background electric field. Streltsov and Pedersen [2010] showed with numerical simulations that IFI develops significantly faster when the heating occurs with a constant beam (not modulated in time with any frequency) and the spot is moving in the direction of the background electric field with the phase velocity of the wave. This velocity can be approximately estimated from the ion mobility and the magnitude of the electric field in the ionosphere. More accurately it can be defined from the simulation of the coupled, nonlinear magnetosphere-ionosphere models. This approach can be considered as a modification of the geometric modulation technique discussed by Cohen et al. [2011] and a similar approach for generation ULF/ELF waves had been proposed by Papadopoulos et al. [1994] and Borisov et al. [1996], although they did not take IFI into considereations.

Another interesting example of what happens with the ionosphere when it is heated with the constant beam pointing at the same spot is discussed in the paper by Streltsov and Pedersen [2011]. This study provided an alternative explanation for luminous structures in the form of rings or solid spots registered with all-sky cameras during heating experiments at HAARP with O mode, 2.85 $\mathrm{MHz}$ waves propagating in the magnetic zenith. These luminous structures have been discussed in detail by Pedersen et al. [2009], who suggested that they are produced by the refraction from localized density enhancements in the ionosphere caused by the heating.

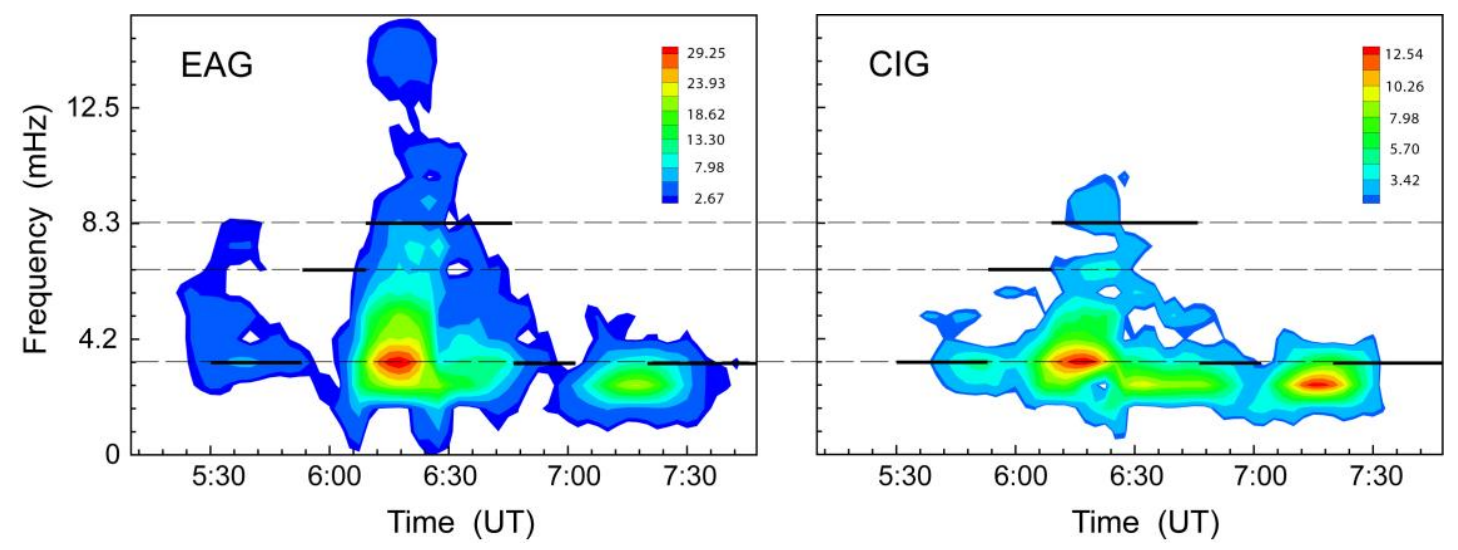

Figure 4.21. Temporal variations of amplitude spectra of the H-component of the magnetic field measured by fluxgate magnetometers during October 29, 2008 HAARP heating experiment at Eagle (EAG) and College (CIG) stations in Alaska. Dashed horizontal lines indicate the modulation frequencies used during the heating experiment [Streltsov et al., 2010a].

Three-dimensional simulations of shear Alfvén waves by Streltsov and Pedersen [2011] suggested that in addition to that effect, density in the ionosphere can also be enhanced locally by the precipitation of the magnetospheric electrons caused by ULF waves standing along the magnetic field lines inside the FLR. These waves can be generated by the ionospheric heating via changing plasma density/conductivity in the D/E region in the presence of the electric field (Xmode heating) or via producing variations in the plasma pressure in the $\mathrm{F}$ region (O-mode heating) 
[Papadopoulos et al., 2011a].

\subsubsection{ULF Waves in the Ionospheric Alfvén Resonator}

The ionospheric feedback instability triggered/controlled by the ground transmitters like HAARP can also generate large-amplitude ULF/ELF waves with frequencies $0.1-10.0 \mathrm{~Hz}$ inside the ionospheric Alfvén resonator, which is the cavity in the low magnetosphere between the conducting bottom of the ionosphere (normally, the ionospheric E region) and the strong gradient in the Alfvén speed at the altitude 0.5-1.0 $R_{E}$ [Polyakov and Rapoport, 1981].

The basic physics of IAR has been extensively studied both theoretically and experimentally [e.g., Belyaev et al., 1990; Trakhtengertz and Feldstein, 1984] and several attempts had been made to excite ULF waves inside IAR with ground based transmitters. For example, generation of Pc-1 (-s period) waves was studied in detail by Bösinger et al. [2000] who suggested that IAR needed to be included in the model to explain some of the results. In this frequency range electron temperature modulation still plays the dominant role in modifying the conductivity rather than the electron density. A unique event was the excitation of waves of $3 \mathrm{~Hz}$ in the ionospheric Alfvén resonator, detected both by magnetometers on the ground and in modulated electron fluxes seen on the FAST satellite [Robinson et al., 2000]. Realistic modelling of the event showed that an Alfvén wave could be generated whose parallel electric field at the top of the ionospheric Alfvén resonator could accelerate electrons to suprathermal energies as observed on the satellite [Kolesnikova et al., 2002; Wright et al., 2003]. Unfortunately, a similar excitation could not be repeated in spite of several attempts.

Scoffield et al. [2006] excited the IAR using the Space Plasma Exploration by Active Radar (SPEAR) high power facility on Svalbard $\left(78.15^{\circ} \mathrm{N}, 16.05^{\circ}\right.$ E). Streltsov et al. [2011] conducted numerical and experimental studies of the excitation of the IAR by heating the ionosphere with HAARP in October-November of 2010. In the later experiments HAARP transmitted HF waves of different frequencies and different polarizations (X-mode and O-mode), vertically and in the direction of the magnetic zenith in daytime and nighttime conditions. The heating was accompanied by comprehensive numerical modeling of the IAR properties for the geophysical conditions observed during the experiments.

Results from these experiments are in a good qualitative agreement with many previous studies of ULF/VLF wave excitation by ionospheric heating [e.g., Papadopoulos et al., 2003; Scoffield et $a l ., 2006]$ and they can be summarized as follows:

1. The excitation of ULF waves is more efficient when the heating is conducted with X-mode waves rather than with O-mode waves. This means that the generation of ULF waves involves modification of the density/conductivity in the D/E region when the electric field exists in the ionosphere.

2. The excitation of ULF waves is more efficient when the heater waves are transmitted in the direction of the magnetic zenith rather than in the vertical direction. 
3. The excitation of ULF waves is more efficient during the nighttime (low conductivity conditions) than during the daytime (high conductivity conditions).

4. The magnetic field measured on the ground has a constant magnitude in the frequency range below $5 \mathrm{~Hz}$ for all modes of excitation.

5. Simulations confirm the results from the observations, and what is most important, they predict that the best way to detect the resonant waves inside the IAR is to measure the electric field at the altitude 500-1000 km above HAARP. This theoretical prediction has been confirmed by direct measurements of the electric field on the DEMETER satellite at an altitude of $670 \mathrm{~km}$ above HAARP during the experiment.

\subsubsection{ULF Waves in the Earth-Ionosphere Waveguide (Schumann Resonator)}

The EISCAT and HAARP transmitters have been used to generate ELF/VLF waves propagating into the earth-ionopshere waveguide. This wide bandwidth source was exploited at EISCAT to test Earth-ionosphere wave guide propagation theory [Barr et al., 1986 and references therein]. At HAARP Maxworth et al., [2015] describes multistation observations of the azimuth, polarization, and frequency dependence of ELF/VLF waves observed on the ground and generated by the electrojet modulation. Cohen et al. [2008] show how the amplitude of the ELF waves detected on the ground depends on the orientation of the HAARP ELF ionospheric dipole relative to the auroral electrojet. Barr et al. [1988] and Cohen et al. [2010] analyzed generation of ELF/VLF waves for long-distance propagation via steerable HF heating of the lower ionosphere and Cohen et al. [2012a] investigate HF beam parameters in ELF/VLF wave generation via modulated heating of the ionosphere. As in the case of ULF waves in the magnetospheric resonators, it is reasonable to expect that the excitation of ELF waves in the earth-ionosphere waveguide also will be more efficient if the frequency of the waves matches the eigenfrequency of oscillations standing inside some resonator cavity. In the earth-ionosphere system one such resonator is the Schumann resonator [Schumann, 1952]. which has been studied in a number of theoretical and experimental papers, suggesting that it can be naturally excited by isolated lightning or electromagnetic radiation from the global thunderstorm activity.

A simple estimation of the fundamental eigenfrequency of the electromagnetic waves trapped inside the spherical cavity formed by the ionosphere and the earth's surface gives a value of 7.5 $\mathrm{Hz}$, and observations from magnetometers around the globe constantly show the enhanced electromagnetic activity in the frequency range from 7 to $9 \mathrm{~Hz}$ which is attributed to the Schumann resonator. The quality of the resonator cavity depends on the parameters of the ionosphere and the resonator can "leak" the electromagnetic power into the magnetosphere where it can be detected by satellites [e.g., Simoes et al., 2011; Surkov et al., 2013]. Because the resonator provides a natural

mechanism for amplification of ELF waves an important question arises, namely, can it be excited artificially?

Attempts were made to generate ELF signals in the frequency range from $6 \mathrm{~Hz}$ to $76 \mathrm{~Hz}$ by 
changing ionospheric conductivity in the polar electrojet region with $1 \mathrm{MW}$ High-Power Auroral Simulation (HIPAS) HF heater facility located near Fairbanks, Alaska [McCarrick et al., 1990]. The main result from this experiment is that the amplitude of the ELF signal measured on the ground depends less on the frequency of modulation but strongly correlates with the level of the electrojet activity, which basically means that the Schumann resonator was not detected during this experiment.

More recently an experiment aimed at the excitation of waves in the Schumann resonator was conducted at HAARP on March 16, 2013 from 00:55 to 01:45 UT [Streltsov et al., 2014]. During that time interval HAARP transmitted 46 one-minute pulses of X-mode waves with frequencies 3.04 MHz, 4.57 MHz, and 6.09 $\mathrm{MHz}$ (the second, third and forth electron gyroharmonics) in the direction of the local magnetic zenith. Each pulse of the heating waves was modulated with one of the frequencies $7.0 \mathrm{~Hz}, 7.2 \mathrm{~Hz}, 7.4 \mathrm{~Hz}, 7.6 \mathrm{~Hz}, 7.8 \mathrm{~Hz}$, or $8.0 \mathrm{~Hz}$.

The experiment produced 46 power spectral densities (PSD) taken every 1-min time interval of the east-west $\left(B_{E W}\right)$ and north-south $\left(B_{N S}\right)$ components of the magnetic field measured near the HAARP site with ground-based magnetometers. All PSDs showed an increase in the amplitude of the electromagnetic power in the range from $7 \mathrm{~Hz}$ to $9 \mathrm{~Hz}$ and eight out of forty-six PSDs show a strong peak in the amplitude at the frequency of the modulation during that particular time interval. Two PSDs of $B_{N S}$ are shown in Figure 4.22: Pannel A shows the case of no resonance and the pannel B shows the case of resonace. Frequencies of modulation of the HF signal is marked with pink vertical lines.

Results from this experiment confirm that the ionospheric heating modulated with frequencies of the Schumann resonance can indeed stimulate relatively large-amplitude electromagnetic response under some particular combination of the heater frequency, modulation frequency, and geomagnetic conditions. (There were no effects from heating in 37 out of 46 considered events.)
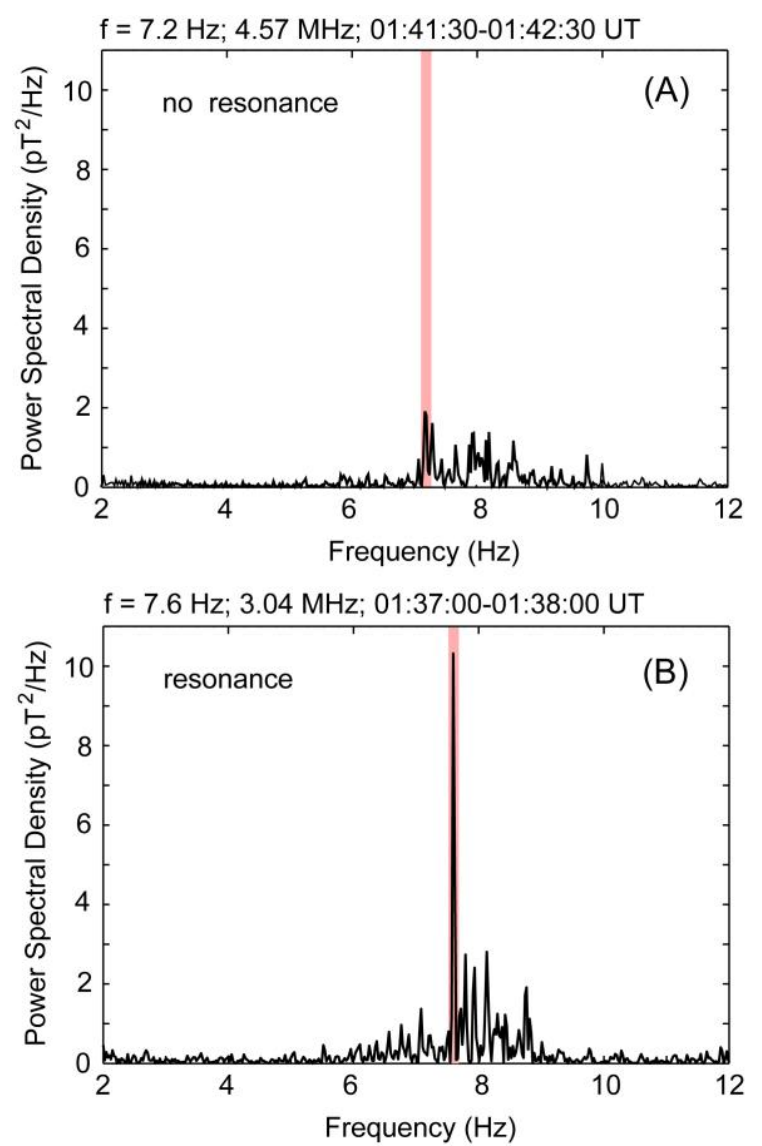

Figure 4.22. PSD of $B_{N S}$ in case of no resonance (panel A) and the PSD of BNS in case of resonance (panel B). Frequencies of modulation of the HF signal is marked with pink vertical lines. (Adapted from [Streltsov et al., 2014].) These conditions include relatively high density in the ionospheric F region above the HAARP and the usage of a pump wave with frequency near the second and the third electron 
gyroharmonics, namely 3.04 $\mathrm{MHz}$ and $4.57 \mathrm{MHz}$.

\subsubsection{ELF/VLF Waves in the Magnetosphere}

Another quite interesting and importnt direction of active ionsopheric experiments with ground HF heaters is injection into the magnetosphere VLF whistler-mode waves. One of the main reasons for interest in these waves is their ability to interact via cyclotron resonance with energetic electrons in the earth's radiation belt. These interactions can change the pitch angle of the energetic particles and remove them from the magnetosphere. This concept is illustrated schematically in Figure 4.23 from Golkowski et al. [2008]. Therefore, controlled injection of whistlers into the magnetosphere from the ground or from space platforms can decrease the number of energetic particles inside the Earth's radiation belts and make the radiation environment there safer for spacecrafts and their human crew [Inan et al., 1985, 2003].

Experiments involving injection of ELF/VLF waves into the magnetosphere from Siple station in Antarctica are described in several books and review papers [e.g., Helliwell et al., 1965; Inan et al., 1985; Helliwell, 1988]]. More recent experiments using a heating facility include:

- Direct measurement of the VLF transmitter signals propagating into the magnetosphere by Cohen and Inan [2012].

- Observations of multi-hop whistler-mode ELF/VLF signals and triggered emissions excited by the HAARP HF heater [Inan et al, 2004].

- Study of the magnetospheric amplification and emission triggering by ELF/VLF waves injected by the 3.6 MW HAARP ionospheric heater [Golkowski et al., 2008].

- Study of cross modulation of whistler mode and HF waves above the HAARP ionospheric heater [Gotkowski et al., 2009].

- Study of amplitude and phase of nonlinear magnetospheric wave growth excited by the HAARP HF heater [Golkowski et al., 2010].

- Study of the occurrence of ground observations of ELF/VLF magnetospheric amplification induced by the HAARP facility [Golkowski et al., 2011].

- Study of the magnetospheric injection of ELF/VLF waves with modulated or steered $\mathrm{HF}$ heating of the lower ionosphere [Cohen et al., 2011].

Under some special conditions, whistlers can propagate along the ambient magnetic field, for example, when they are trapped and guided by magnetic field-aligned density inhomogeneities or ducts. So it is possible to detect the signal in the location magnetically conjugate to the transmitting station and, by analyzing this signal, to make conclusions about parameters of the magnetosphere. A number of such

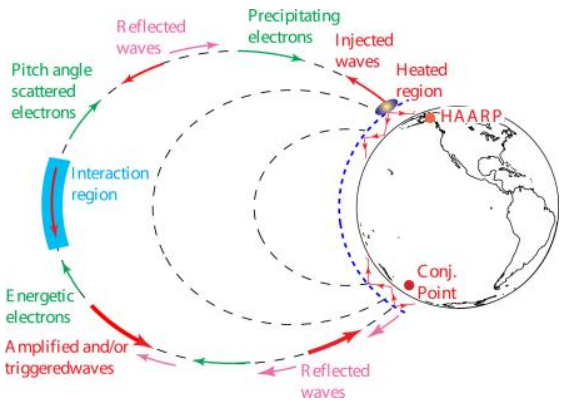

Figure 4.23. Schematic plot illustrating ducted whistler-mode wave propagation excited by the HAARP HF heater (from [Gołkowski et al., 2008]). 
experiments had been conducted with the transmitter located at Siple station in Antarctica and reciever in the magnetically conjugate location, near Roberval and Lake Mistissini in Canada. Magnetically conjugate location for the HAARP transmitter is in the South Pacific Ocean, and there the signal can be received on a radio buoy or on board a research ship [Gołkowski et al., 2008; Streltsov et al., 2010b].

One of the most interesting and important directions of experiments with VLF waves is nonlinear amplification of the initial (triggering) signal and generation of intense secondary emissions. These effects had been observed in a number of experiments conducted at Siple Station in Antarctica [Helliwell et al., 1965; Inan et al., 1985; Helliwell, 1988]. Although experiments where ELF/VLF waves were excited in the ionosphere were very fruitful, with the waves from both high latitude HF facilities being detected in space on various satellites, the hope of exciting wave-particle interactions like amplification and non-linear triggering of emissions was never realized at EISCAT, probably because the source was at too high a latitude, outside the plasmasphere. The lower latitude of HAARP allowed magnetospheric propagation to the conjugate hemisphere more readily [Golkowski et al., 2011] but even at HAARP such results were sparse. Golkowski et al. [2011] find that "It is deduced that the primary variable that is associated with successful ground observations of HAARP-induced magnetospheric amplification is availabilityof magnetospheric wave guiding structures. Such structures are found to be most prevalent under quiet geomagnetic conditions following a disturbance when the plasmapause extends to the latitude of the HAARP facility or higher."

Another partial explanation of this problem is that these effects are produced by non-linear interactions between whistler-mode waves and energetic electrons in the equatorial magnetosphere and they depend on the wave amplitude/power [Helliwell, 1988]. Therefore, the results depend on the efficiency of how the electromagnetic power can be delivered from the ionosphere to the equatorial magnetosphere. This issue is particularly important for the waves generated by the HF transmitters via modulation of the ionospheric conductivity in the electrojet region, because this mechanism is not as efficient as a wave generation done with a very long VLF antenna on the ground/ice, as it was done in experiments at Siple Station [Inan et al., 1985, 2003; Helliwell, 1988]. For example, Barr et al., [1985a] measured the efficiency of generating 1-2 kHz waves was very low with 1-2 W being radiated for an HF input power of about $1 \mathrm{MW}$.

Experimental study of amplification of VLF signal with frequency changing in time had been conducted at HAARP on 16 March 2008 [Streltsov et al., 2010b]. During the experiment HAARP transmitted $2.75 \mathrm{MHz}$ X-mode wave modulated with frequency changing in time from 0.5 to 2.5 $\mathrm{kHz}$ as well as constant frequency pulses at 510, 820, 1250, 1510, 1875, 2125, and $2500 \mathrm{~Hz}$. Ground measurements of ELF/VLF waves were made in the vicinity of the HAARP facility at Chistochina, Alaska, and in the magnetic conjugate region in the South Pacific Ocean on-board the research ship Tangaroa. 
Results from this experiment demonstrate that whistler-mode waves with a particular form of the frequency modulation can be amplified on their pass from HAARP to the conjugate location more efficiently than the signal with a constant frequency. Numerical simulations of the electron MHD equations in the dipole magnetic field geometry revealed that the amplification takes place more efficiently when the frequency of the whistler mode waves (in the frequency range from 0.5 to $1.0 \mathrm{kHz}$ ) changes in the equatorial magnetosphere at the rate of 0.25 to $0.47 \mathrm{kHz} / \mathrm{s}$. The maximum amplification occurs when this rate is $0.33 \mathrm{kHz} / \mathrm{s}$, and no/very little amplification was observed when this gradient is equal to 0 or when it is larger than $0.78 \mathrm{kHz} / \mathrm{s}$ [Streltsov et al., 2010].

Results from this experiment and corresponding numerical simulations are consistent with results of earlier experiments at Siple station. In these earlier experiments it was shown that signal amplification and triggering were not observed when two signals with a frequency difference less than $20 \mathrm{~Hz}$ were launched together, yet signals with a frequency difference of 100-200 Hz were amplified [Helliwell, 1988]. The mechanism causing this amplification of the monochromatic signals was called the coherent wave instability. It suggests that true broadband signals are not amplified in the magnetosphere, and this situation can be avoided if the frequency of the transmitted wave changes not in a smooth, continuous format but rather in a discrete, "staircaselike" form with a step of 100-200 Hz.

There are also significant differences between earlier experiments at Siple Station and the March 16, 2008 experiment at HAARP. Siple's experiments showed several cases of amplification of signals with a linear frequency modulation (rising frequency) and signals with a frequency variation that have a positive slope but a negative curvature ("chirp-like" signals). It was observed that these "chirp-like" signals amplify more rapidly than the signals with linear frequency variation. HAARP's experiment demonstrates amplification of the signal with a positive frequency slope and a positive curvature, which make it different from the experiments conducted at Siple.

\subsection{Descending Artificial Ionization Layers (DLs)}

Carlson [1993] predicted that artificial ionization would occur at ERP $P_{0}$ comparable to the solar ultraviolet radiation creating the natural F-region ionosphere, i.e., $P_{0} \sim 1 \mathrm{GW}$. The artificial plasma patches ("layers") descending from the initial interaction altitude were first identified in the Pedersen et al. [2010] experiments at HAARP with $P_{0} \approx 0.45 \mathrm{GW}$, albeit descending radar, optical, and SEE features were observed earlier at EISCAT [Djuth et al., 1994; Dhillon and Robinson, 2005; Ashrafi et al., 2007]. The $427.8 \mathrm{~nm}$ emissions from the descending plasma and the coincident ion line echoes implied ionization by suprathermal electrons accelerated in the descending plasma resonance. Therefore, the DL formation has been explained as an ionizing wavefront created by the SLT-accelerated electrons [Mishin and Pedersen, 2011; Eliasson et al., 2012b; 2015]. It is instructive to give the basics of this self-similar process [Mishin 
and Pedersen, 2011] before providing the observational details and their interpretation.

\subsubsection{Ionizing Wavefront}

The accelerated, ionizing $\left(\varepsilon>\varepsilon_{\text {ion }}\right)$ electrons with the density $n_{t}^{\text {ion }}$ move along the magnetic field forming a tongue of freshly-created ionization. Let us assume that the plasma resonance condition, $n_{e}=n_{c}$, is met at some altitude $h_{c}$ near the tongue's tip below the initial resonance $h_{c}^{0}$. That is, the interaction region is shifted downward. The descent is slow with respect the acceleration process, so the latter can be considered as in stationary plasma (section 3.7). As long as the ionization rate $q_{t}^{i o n}$

$$
\frac{d n_{e}}{d t}=q_{t}^{i o n} \sim n_{t}^{i o n} \cdot\left\langle v_{i o n}(\varepsilon)\right\rangle,
$$

is greater than that of recombination and diffusion, the artificial plasma moves downward selfsimilarly at a speed $v_{D}=d h_{c}(t) / d t$. Here $\left\langle v_{i o n}\right\rangle \approx 2 \cdot 10^{-8}\left(\left[N_{2}\right]+\frac{1}{2}[O]\right) \mathrm{s}^{-1}$ is the ionization frequency [Majeed and Strickland, 1997] averaged $(\langle\ldots\rangle)$ over the accelerated distribution $F_{t}$ with $\varepsilon_{\max }>>\varepsilon_{i o n}$ and $\left[N_{2}\right] /[O]$ is the density of nitrogen/oxygen - the main constituents of air with the total density $N_{n} \approx\left[N_{2}\right]+[O]$.

Let us designate the $\left(\| \mathbf{B}_{0}\right)$ extent of the overdense plasma below $h_{c}(t)$ as $L_{i o n}(t)$, that is $n_{e}\left(h_{c}(t)-L_{i o n}(t)\right)=n_{c}$. For $\varepsilon_{\max } \leq 100 \mathrm{eV}$, the coefficient of inelastic losses is small, $\delta_{i l}(\varepsilon)=v_{i l}(\varepsilon) / v_{e}(\varepsilon)<<1$. As the ionizing electrons undergo many $\left(\sim \delta_{i l}^{-1}\right)$ elastic collisions before ionization, the ionization length reduces to $L_{i o n} \sim\left\langle v \delta_{i l}^{1 / 2} / v_{i o n}\right\rangle$. As $n_{c}>>n_{0}$ at altitudes below the initial resonance, one gets $\tau_{i o n}^{-1} \sim q_{t}^{i o n} / n_{c}$ and the speed of descent

$$
v_{D} \sim L_{i o n} / \tau_{i o n} \sim\left\langle v_{i o n}\right\rangle\left\langle\delta_{i l}^{1 / 2} \frac{v}{v_{i o n}}\right\rangle \frac{n_{t}^{i o n}}{n_{c}} \sim 10^{6} \frac{n_{t}^{i o n}}{n_{c}}[\mathrm{~m} / \mathrm{s}]
$$

Taking $n_{t}^{\text {ion }} \sim 3 \cdot 10^{-4} n_{c}$ matches the observed values of the fast descent $v_{D} \sim 0.3 \mathrm{~km} / \mathrm{s}$ and the $427.8 \mathrm{~nm}$ emission $\sim 10 \mathrm{R}$ (Figure 4.29c-d). As the plasma loss is neglected, equation (2) contains no direct dependence on the neutral density so that $v_{D}(h)$ is constant if $n_{t}^{\text {ion }}=$ const along the path. Eliasson et al.'s [2012b; 2015] simulations show that at $n_{t}^{\text {ion }} \approx(2 \rightarrow 6) \cdot 10^{-4} n_{c}$ the descent stops at $h_{D}^{*} \approx 180 \rightarrow 150 \mathrm{~km}$ due to the increase of recombination and diffusion rates. Besides, the decrease of the SLT extent, $\quad l_{L T} \propto l_{A} \propto L_{i o n}^{1 / 3} \propto N_{n}^{-1 / 3} \quad$ (section 3.1) reduces the transit time $\tau_{a} \sim l_{L T} / \varepsilon_{\max }^{1 / 2}$ and thus $\varepsilon_{\max }$. Further, accounting for inelastic losses, $\operatorname{St}_{t}(\varepsilon) \approx-v_{i l}(\varepsilon) F_{t}(\varepsilon)$, of the accelerated electrons puts additional bounds on $\varepsilon_{\max }$ at $h_{D} \leq 160 \mathrm{~km}$ [Mishin and 
Pedersen, 2011; Eliasson et al., 2012b]. That is, when $v_{i l}(\varepsilon)$ exceeds the acceleration rate $\sim \omega_{p e}^{2}\left|E_{\frac{\alpha_{p e}}{u}}\right|^{2} / 32 \pi n_{e} \varepsilon^{3 / 2}$, acceleration stops at $\varepsilon_{\max }<100 \mathrm{eV}$ [Volokitin and Mishin, 1979]. Both effects reduce $n_{t}^{\text {ion }}$ at low altitudes. Another factor is that the pump propagation is affected, particularly at altitudes where $n_{0}\left(h_{c}\right)<<n_{c}$ and the O-mode index of refraction is mainly defined by the DL plasma [Gurevich et al., 2002].

\subsubsection{Observations of DLs}

Incoherent Radar Backscatter

Persistent descending PL and IL echoes were revealed for the first time by Djuth et al. [1994] during daytime injections of $6.77 \mathrm{MHz}$ waves at $P_{0}=0.4 p_{0}=1.1 \mathrm{GW}$ with a 1-min/2-min on/off duty cycle at $\theta_{0} \leq 5^{\circ} \mathrm{S}\left(<\theta_{s}\right)$. A half-power $(-3 \mathrm{~dB}) \mathrm{HF}$ transmitter beam width was $\Delta \theta_{t r} \approx 4^{\circ}$. The EISCAT UHF incoherent scatter radar $\left(\Delta \theta_{r} \approx 0.5^{\circ}\right)$ detected PL and IL echoes from the same direction with integration time of $1 \mathrm{~s}$. Dhillon and Robinson [2005] explored IL backscatter using the $\mathrm{HF}$ beam at $7.1 \mathrm{MHz}$ with a $2 \mathrm{~min} / 2 \mathrm{~min}$ on/off duty cycle at $0.6-0.9 \mathrm{GW}\left(\Delta \theta_{t r} \approx 6^{\circ}-7^{\circ}\right.$, $p_{0}=1.5-2.25$ ) alternated between vertical (V) and MZ. The EISCAT UHF radar observed IL backscatter from five pointing directions in the magnetic meridian plane with $5 \mathrm{~s}$ averaging.

Figure 4.24 (left frame) exemplifies the PL backscatter in the Djuth et al. [1994] experiments. It is seen that a $\sim 20-\mathrm{dB}, \approx 2-\mathrm{s}$ overshoot is centered near $h_{0}^{0}=210 \mathrm{~km}$, with an altitudinal spread $\sim 750 \mathrm{~m}$. The overshoot turned into a $\sim 1 \mathrm{~km}$-thick layer of $\sim 10 \mathrm{~dB}$ echoes descending at a speed $v_{\text {re }} \sim 150 \mathrm{~m} / \mathrm{s}$ to the
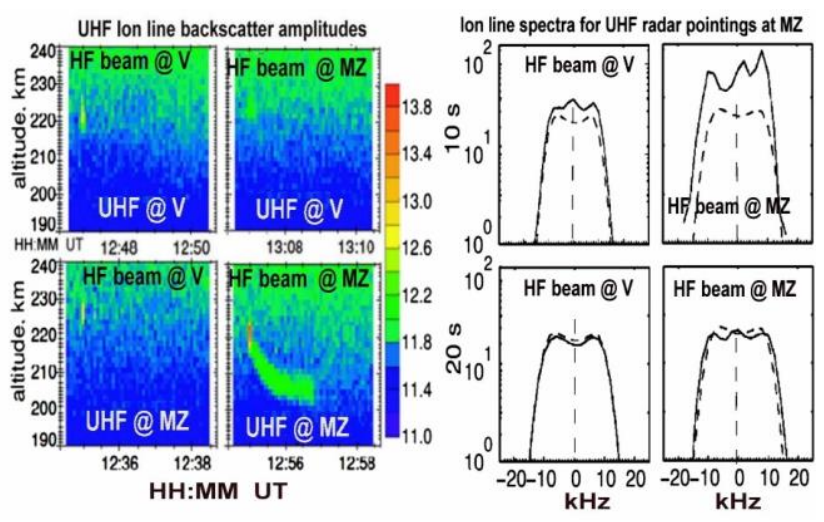

Figure 4.24. (left frame) The upshifted PL backscatter power versus altitude and time (a (signal + noise)-to-noise ratio in $\mathrm{dB}$ is color coded). Adapted from Djuth et al. [1994]. (mid frames) IL backscatter amplitudes in log scale for (left) vertical and (right) MZ injections. Adapted from Dhillon and Robinson [2005]. (right frames) IL Doppler spectra for UHF radar pointing at MZ for V (left) and MZ (right) injections. The solid (dashed) lines correspond to the descending (topside) backscatter during heater on (off) periods averaged over two successive 5-s data dumps. Adapted from Mishin et al. [2016].

terminal altitude $h_{r e}^{*} \approx 206.5 \mathrm{~km}$. Henceforth, the subscript " $r e$ " stands for "radar echoes". Then, the layer weakens and becomes more structured, slowly retreating upward until the heater is turned off. Cloudlike structures in the range-time-intensity plot indicate random small-scale irregularities. The central peak in the IL Doppler spectra [Djuth et al., 1994] indicates the SLT regime in the 
descending region. During power stepping with $5 \mathrm{~s}$ steps of $2.5 \%, 5 \%, 10 \%, 25 \%, 50 \%$, and $100 \%$ of full power, the layers start descending at $25 \%$, with the speed increasing with the power.

Contrary to the descent for $6.77 \mathrm{MHz}$, vertical injections at $7.1 \mathrm{MHz}$ produce only $\sim 10-15 \mathrm{~dB}$ overshoots in the first data dump, with the central (SLT) Doppler spectral peak. For MZ injections, $\sim 20 \mathrm{~dB}$ overshoots at $\mathrm{MZ}$ near $h_{0}^{0}=220 \mathrm{~km}$ turn into a few km thick layer of $\sim 10 \mathrm{~dB}$ backscatter descending to

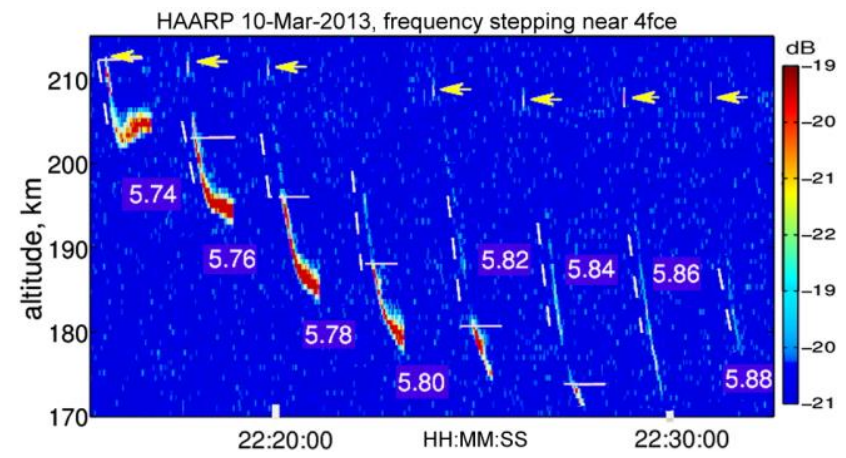

Figure 4.25. Plasma line backscatter power in $\mathrm{dB}$ from MUIR at $f_{0}$ from 5.74 to $5.88 \mathrm{MHz}$. Solid horizontal and dashed oblique lines indicate the gyroresonance hgr and the descent rates, respectively. Arrows show initial overshoots. (From Mishin et al. [2016]). $h_{r e}^{*} \approx 206 \mathrm{~km}$ in $\sim 1 \mathrm{~min}$. The layer persists near $h_{r e}^{*}$ until the heater is turned off. The electron temperature increased by about three times during MZ injections [cf. Rietveld et al., 2003]. The central Doppler spectral peak during descent indicates SLT in the descending layer (the spectra remain after $20 \mathrm{~s}$ ). The greater up-shifted "shoulder" means greater amplitudes of downwardpropagating ion acoustic waves. Unfortunately, a low time resolution prevents specifying the onset of the DL formation. Note that $6.77 \mathrm{MHz}$ is within a few $\mathrm{kHz}$ of $5 f_{c e}$ during descent, while 7.1 $\mathrm{MHz}$ is about $5.2 f_{c e}\left(h_{r e}^{*}\right)$.

Subsequent experiments at HAARP have shown descending features both above and below the gyroresonance $h_{g r}\left(s f_{c e}\left(h_{g r}\right)=f_{0}\right)$. Figure 4.25 shows the power of field-aligned upshifted PL echoes measured by MUIR $\left(\Delta \theta_{r} \approx 8.6^{\circ}\right)$ with 600 $\mathrm{m}(10 \mathrm{~ms})$ range (time) resolution on 10 March 2013. The critical frequency $f_{O} F 2$ was $\approx 7.2 \mathrm{MHz}$ indicating steady daytime conditions. O-waves at frequencies stepping by $20 \mathrm{kHz}$ from 5.74 to 5.88 $\mathrm{MHz}$ were injected into $\mathrm{MZ}$ at $P_{0} \approx 1.8 \mathrm{GW}$ ( $\Delta \theta_{t r} \approx 7^{\circ}, p_{0}=4.5$ ), with a 2-min cycle (1 min on/off). The heights of $\sim 1$-s PL overshoots (arrows) are well above $h_{g r}$ (horizontal lines) at all frequencies except 5.74 MHz. At 5.76-5.88 MHz, persistent signals with an altitudinal spread of $\sim 2$ $3 \mathrm{~km}$ start after about $0.1-0.2 \mathrm{~s}$ at $\sim 5-10 \mathrm{~km}$ below

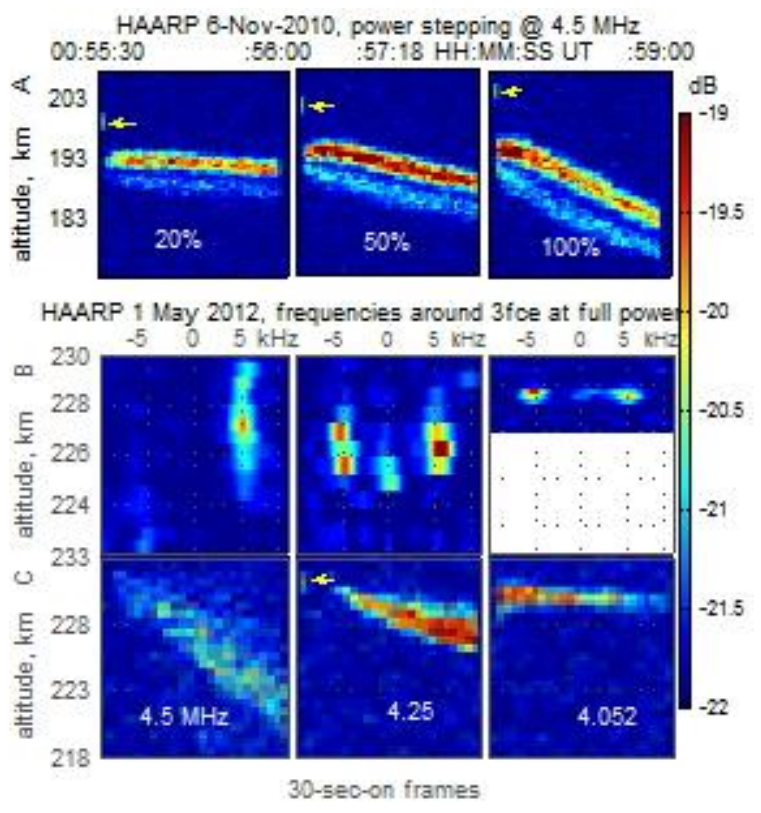

Figure 4.26. (A) Ion line backscatter power colorcoded in $\mathrm{dB}$ for MZ injections with ERPs ranging from $20 \%$ to $100 \%$ of $1.1 \mathrm{GW}$. (B) Doppler IL spectra during MZ injections at full HF power at 4.5, 4.25, and $4.052 \mathrm{MHz}$ corresponding to altitude-time plots in row C. [Mishin et al., 2016]. 
the overshoots but above $h_{g r}$. As $h_{r e}(t)$ passes through $h_{g r}$, the descent continues with reduced speeds and greater PL amplitudes. Near the terminal point, the PL amplitude at $\leq 5.84 \mathrm{MHz}$ increases further and the layer swells.

Figure 4.26A shows the IL backscatter power from MUIR for $20 \%$, 50\%, and $100 \%$ of full power $P_{0} \approx 1.1 \mathrm{GW}\left(\Delta \theta_{t r} \approx 12^{\circ}, p_{0}=2.75\right)$ during $30 \mathrm{~s} \mathrm{MZ}$ injections at $4.5 \mathrm{MHz}\left(h_{r e}>h_{g r}\right)$ on 6 November 2010. Overshoots near $h_{m z}$ appear even at $1 \%$ of $P_{0}$ and typically last for about $0.2 \mathrm{~s}$. However, similar to Djuth et al. [1994], persistent descending echoes appear at $\geq 0.2 \mathrm{GW}$, with greater speeds at higher ERPs $\left(v_{r e} \approx 400 \mathrm{~m} / \mathrm{s}\right.$ at $100 \%)$. This is a unique observation of two distinct broad layers of IL echoes that closely follow each other, with the lower layer well below the $\mathrm{PD}_{L}^{O}$ matching height. The backscatter power in the upper layer at $100 \%$ is weaker than at $50 \%$ but the speed of descent is greater. Surprisingly, the upper/lower layer contains only the positive/negative Doppler-shifted spectral peak.

Shown in Figures 4.25B and 4.25C are ionline power spectra observed in MZ on 1 May 2012 for $\mathrm{HF}$ frequencies above and below $3 f_{c e} \approx 4.32$. Altitude-frequency Doppler spectra in row B are obtained in the middle of the corresponding altitude-time backscatter power plots in row $\mathrm{C}$ (averaged for $0.5 \mathrm{~s}$ ). Strong broad signals at positive Doppler and much weaker signals at negative Doppler are seen at different altitudes for 4.5 MHz. The usual two decay peaks with

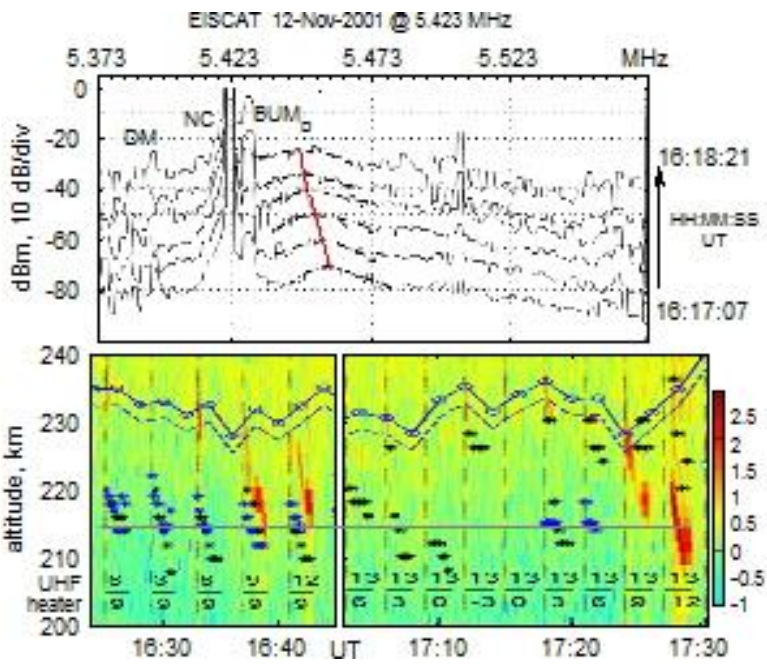

Figure 4.27. (top) Consecutive SEE spectra from 16:17 to $16: 18: 21$ UT. The red line indicates the peak BUM frequency. (bottom) Raw ion-line backscatter power in $\log$ scale during the first (left) and second (right) half of the experiment with the green-line optical height triangulation results (black asterisks) and $\mathrm{h}_{\text {bum }}$ (blue asterisks) superimposed. Black dashes indicate the pump-on periods. The upper (lower) labels mark the UHF radar (HF pump) zenith angles. The blue solid (dashed) and dark solid lines indicate $\mathrm{h}_{0}\left(\mathrm{~h}_{\mathrm{uh}}\right)$ and the gyroresonance $\mathrm{h}_{\mathrm{gr}} \approx 215 \mathrm{~km}$, respectively. (Adapted from Ashrafi et al. [2007].)

Doppler shifts about $\pm 5 \mathrm{kHz}$ at the same altitude

and a zero-Doppler central (SLT) peak are seen at 4.25 and 4.052 MHz. Persistent weak signals for $4.5 \mathrm{MHz}$ with an altitudinal spread of $\approx 3 \mathrm{~km}$ descend at a greater speed than stronger signals for $4.25 \mathrm{MHz}$.

\section{SEE, $557.7 \mathrm{~nm}$ Emissions and Ion Line Backscatter}

Descending optical and SEE emissions have been revealed by Ashrafi et al. [2007] during injections at $5.423 \mathrm{MHz}\left(4 f_{c e}\right.$ at $\left.h_{g r} \approx 215 \mathrm{~km}\right)$ and $P_{0}=0.55 \mathrm{GW}\left(p_{0}=1.375\right)$. The $\mathrm{HF}$ radio beam $\left(\Delta \theta_{t r} \approx 7^{\circ}\right)$ was initially centered at $9^{\circ} \mathrm{S}$ and had a 2-min on/off cycle until 16:55 UT. 
Then, it was scanned in $3^{\circ}$ steps between $3^{\circ}$ north and $15^{\circ} \mathrm{S}$ of vertical with a $2 / 1-$ min on/off cycle. Data come from measurements of the optical emissions at two separate sites, SEE with a $14 \mathrm{~s}$ integration time and $300 \mathrm{~Hz}$ frequency resolution, and EISCAT UHF radar ion-line backscatter with a $5.4 \mathrm{~km}$ range resolution and $5 \mathrm{~s}$ integration time. The radar was sweeping in $3^{\circ}$ steps from $3^{\circ} \mathrm{N}$ to $15^{\circ} \mathrm{S}$ of vertical in a north-south meridian scan during 15:08-16:55 UT and MZ-pointed later on. The triangulated height, $h_{g l}$, of the green-line emission has the average uncertainty of $4-$ $5 \mathrm{~km}$.

Figure 4.27 (top) shows the consecutive SEE spectra with added 10-dB offsets for the heating cycle starting at 16:17 UT. The DM, BUM, and NC features are indicated. The BUM peak frequency decreases with time at the rate $r_{f} \approx 0.2 \mathrm{kHz} / \mathrm{s}$ indicating the increase of $f_{c e}(t)$ so that $4 f_{c e}(t) \rightarrow f_{0}$. That is, the BUM generation altitude $h_{b u m}$ descends as $f_{c e}\left(B_{0}\right)$ increases downward. This spectral feature is therefore called the $B U M_{D}$ ("D" stands for "descending"). Using the IGRF model, the descent speed is calculated as $v_{\text {bum }} \approx r_{f}\left(4 d f_{c e} / d h\right)^{-1} \approx 90 \quad \mathrm{~m} / \mathrm{s}$. Altitude-time plots of raw IL backscatter power for several consecutive HF pump cycles (bottom) illustrate that the radar, SEE, and optical features are coincident. Note the persistent narrow continuum (NC), which indicates the SLT development near the plasma resonance. The matching altitudes $h_{g r}, h_{u h}, h_{0}$ and $h_{\text {bum }}$ are calculated using the ionosonde data and IGRF model.

Overall, enhanced IL echoes persist in $\mathrm{MZ}\left(12^{\circ} / 13^{\circ} \mathrm{S}\right)$ and $9^{\circ} \mathrm{S}$ radar positions for the $\mathrm{HF}$ beam at $9^{\circ} \mathrm{S}$ and MZ, while only 10-15 s overshoots are seen for the other angles. The $B U M_{D}$ feature remains throughout. With the HF beam scanning, clear and consistent $B U M_{D}$ was seen only near 17:20 UT at $3^{\circ} \mathrm{S}$ and $6^{\circ} \mathrm{S}$ just above $h_{g r}$. Descending

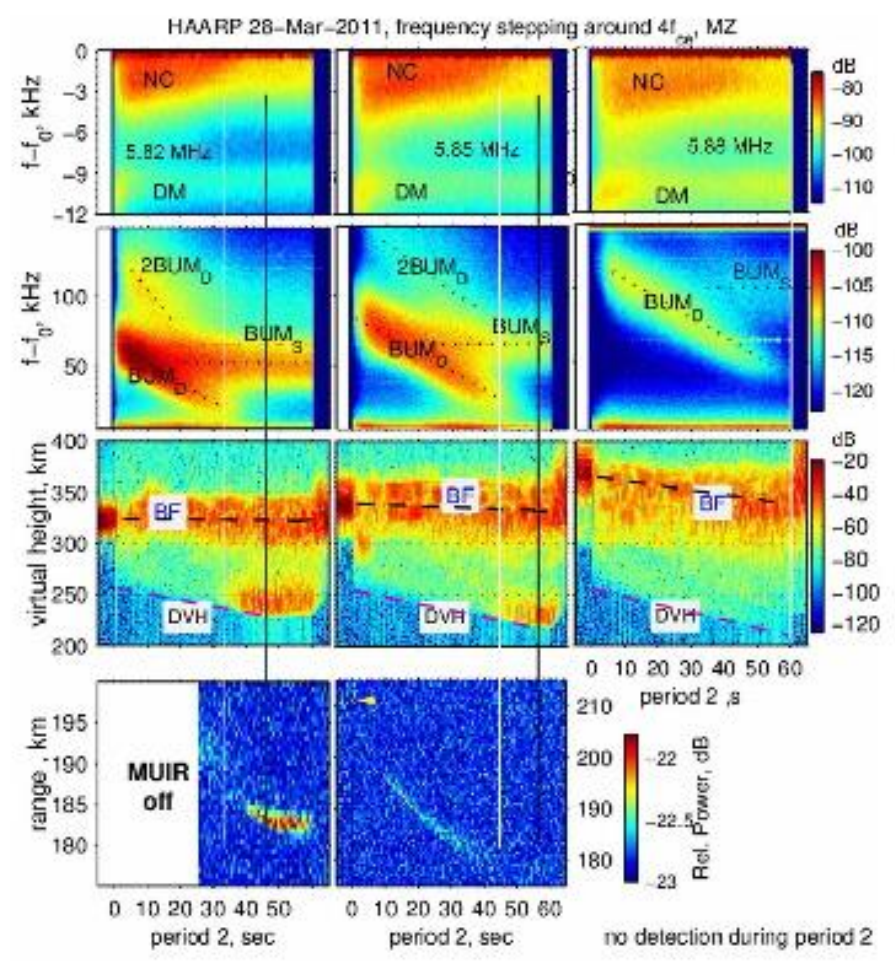

Figure 4.28. (top) SEE frequency-time spectrograms zoomed near $\mathrm{f}_{0}$ with $\mathrm{NC}$ and $\mathrm{DM}$ indicated, $\left(2^{\text {nd }}\right) 0-150 \mathrm{kHz}$ SEE spectrograms with $\mathrm{BUM}_{\mathrm{S}}, \mathrm{BUM}_{\mathrm{D}}$, and $2 \mathrm{BUM}_{\mathrm{D}},\left(3^{\text {rd }}\right)$ virtual (reflection) heights of diagnostic pulses with $\mathrm{BF}$ and $\mathrm{DVH},\left(4^{\text {th }}\right)$ height-time plots of the PL intensity integrated for $0.5 \mathrm{~s}$. The arrow points to a $\sim 2$-s overshoot at the onset of period 2 . The origin of the time and frequency axes is at the start of period 2 and the pump frequency $f_{0}$, respectively. Color codes show the intensities in $\mathrm{dB}$. The vertical white (black) lines indicate $\mathrm{t}^{*}$ bum ( $\left.\mathrm{t}^{*} \mathrm{uh}\right)$. After Sergeev et al. [2013] and Mishin et al. [2016]. 
green-line emissions are observed for all injection angles except $3^{\circ} \mathrm{N}$. On average, layers of persistent IL echoes spread over $\sim 3 \mathrm{~km}$ and descend at a speed $120-150 \mathrm{~m} / \mathrm{s}$ until the heater is turned off. The terminal heights $h_{r e}^{*}$ and $h_{g l}^{*}$ are below $h_{g r}\left(<h_{b u m}^{*}\right)$. Although IL Doppler spectra are unavailable, the NC persistence suggests that the enhanced IL echoes come from the descending SLT region. Note that the SEE observations during the 10 March 2013 experiment at HAARP [Sergeev et al., 2013] show the $B U M_{D}$ spectrum similar to Figure 4.27.

\section{SEE, Reflected Probing Signals and Plasma Line Backscatter}

Sergeev et al. [2013] explored SEE and reflected probing signals from three broadband HF receivers at distances $\approx 11(\mathrm{~A}), 83(\mathrm{~B})$, and 113 (C) $\mathrm{km}$ to the south of the HAARP facility and field-aligned PL backscatter from MUIR. Sites A and B were nearly under the heating region at vertical and MZ injections, respectively. The HF beam at 5.73 to $5.88 \mathrm{MHz}$, stepping up by 30 $\mathrm{kHz}$ every $5 \mathrm{~min}$, was pointed at vertical for the first $30 \mathrm{~min}$ and at $\mathrm{MZ}$ for another $30 \mathrm{~min}$ on 28 March 2011. Each step ends by $30 \mathrm{~s}$ off and includes low-duty $30 \mathrm{~s}$ period 1 and $180 \mathrm{~s}$ period 3 and high-duty $1 \mathrm{~min}$ period 2, comprising 20/980 $\mathrm{ms}$ and 160/40 ms on/off cycles at $1.8 \mathrm{GW}$ ( $p_{0}$ $=4.5$ ), respectively. In addition, diagnostic pulses of $0.1 \mathrm{~ms}$ were transmitted in the middle of each $40 \mathrm{~ms}$ pause of period 2. Overall, the descending signatures are observed only for MZ injections at all frequencies except $5.73 \mathrm{MHz}\left(<4 f_{c e}\left(h_{0}^{0}\right) \approx 5.75 \mathrm{MHz}\right)$ [Sergeev et al., 2013]. The results are practically identical at each site, thus indicating broad SEE and scattering patterns.

Figure 4.28 presents the period 2 data from site $\mathrm{B}$ for the last three steps at $f_{0}=5.82,5.85$ and $5.88 \mathrm{MHz}$ when MUIR was turned on. Shown from top to bottom are SEE frequency-time spectrograms (the frequency/time resolution of $200 \mathrm{~Hz} / 0.2 \mathrm{~s}$ ) just below $f_{0}$, with the $\mathrm{NC}$ and $\mathrm{DM}$, and over the range $0-150 \mathrm{kHz}$, with the stationary and descending BUM signatures, virtual heights of scattered diagnostic pulses, with the quasi-stationary (BF) and descending (DVH) signatures, and relative power of MUIR plasma line echoes vs. altitude. The BF layer is due to scattering from the bottomside F2 region, initially centered at $h_{b f}^{0} \approx 325$ (at $5.82 \mathrm{MHz}$ ), $340(5.85 \mathrm{MHz}$ ), and 360 $(5.88 \mathrm{MHz}) \mathrm{km}$, as for the low-duty periods 1 and 3. Descending virtual height (DVH) layers around $h_{d v h}(t)$ appear just after the onset of period 2. The increase in $h_{b f}^{0}$ due to the rise of the F2 layer is consistent with PL overshoots during low-duty period 1 at $h_{m z}^{0} \approx 203 \mathrm{~km}$ (at 5.82 $\mathrm{MHz}$ ), $206 \mathrm{~km}$ (at $5.85 \mathrm{MHz}$ ), and $213 \mathrm{~km}$ (at $5.88 \mathrm{MHz}$ ) [Sergeev et al., 2013]. The BF/DVH broad scattering pattern and cloud-like structure suggest scattering off randomly distributed smallscale irregularities. This is consistent with the disappearance of the DVH layer and the recovery of the BF to that of period 1 in just a few seconds after the end of high-duty period 2 [Sergeev et al., 2013].

The $B U M_{D}$ frequency drift rate is $\gamma_{f} \approx 1.2-1.4 \mathrm{kHz} / \mathrm{s}$ for all $f_{0}$. It gives the speed of descent 
$v_{\text {bum }}=\left(4 d f_{c e} / d h\right)^{-1} \gamma_{f} \approx 450-500 \mathrm{~m} / \mathrm{s}$. The $B U M_{D}$ terminal time, $t_{\text {bum }}^{*}$, increases with $f_{0}$ and so does $h_{\text {bum }}^{0}-h_{\text {bum }}^{*}=v_{\text {bum }} t_{\text {bum }}^{*}$. The DVH layers stop at $t_{d v h}^{*} \approx t_{b u m}^{*}+(10-15) \mathrm{s}$, consistent with the PL signal at 5.82 MHz. The DVH-signals at 5.82 and 5.85 MHz and the PL signal at 5.82 MHz swell and retard below $h_{b u m}^{*} \approx h_{g r}$ until the end of period 2. This, as well as a larger descent speed and weaker PL amplitude at $5.85 \mathrm{MHz}$, is similar to Figure 4.25.

The development of both DVH and PL is consistent with the NC's (top). Namely, the spectral width of the NC brightest (reddish) part, $\delta f_{n c}$, gradually decreases with time approaching $\sim 0.5-$ $\mathrm{kHz}$ band at $\approx t_{d v h}^{*}$ for 5.82 and $5.85 \mathrm{MHz}$, while remaining $\sim 3 \mathrm{kHz}$ for the entire period 2 at 5.88 MHz. The NC power $\left.P_{n c} \propto \delta f_{n c}\right)$ at vertical exhibits the overshoot ( $\left.\sim 10 \mathrm{~s}\right)$ behavior and is much weaker than at MZ [Mishin et al., 2016]. The DM appears almost "instantly" at vertical and MZ and persists for the entire period 2, with the amplitude slightly stronger at MZ. The obvious dominance of NC at MZ (descent) over that at vertical (no descent) suggests the principal role of SLT in the descent.

\section{Ionograms, Optical Emissions and Ion Line Backscatter}

Figure 4.29 summarizes the Pedersen et al. [2010; 2011] experiments at full power $P_{0}=0.44$ $\mathrm{GW}\left(p_{0}=1.1\right)$ near the second gyroharmonic. Fast $(10 \mathrm{~s})$ ionograms from the HAARP ionosonde give matching altitudes $h_{u h}(t)$ (green lines), $h_{0}(t)$ (red), and $h_{2,3}(t)$ (black) for $f_{p e}(\mathrm{t})=2$ and $3 \mathrm{MHz}$ every $1 \mathrm{~min}(10 \mathrm{~s})$ in March (November) 2009. On 17 March 2009, the HF beam at $2.85 \mathrm{MHz}\left(h_{g r}\right.$ $=230 \mathrm{~km}$ ) was pointed in MZ with a 4-min on/off cycle from 05:05 to 05:21 UT and then continuously. Shown is a sequence of images from the remote and HAARP sites during 05:1305:17 UT, a tomographic cross-section of the volume emission rate at $557.7 \mathrm{~nm}$ in the magnetic meridian plane at 15:16:30 UT, and average calibrated intensities at $427.8 \mathrm{~nm}$ for the central region of the images corresponding to the overdense ionosphere. However, altitude-time plots from the remote imager and true height profiles inverted from ionogram at 05:26 UT show also the DL development in the underdense background ionosphere. The spatial coherency of the 557.7 and $427.8 \mathrm{~nm}$ emissions from the HAARP imager implies that the descending plasma is produced by $>18.75 \mathrm{eV}$ electrons.

By and large, the DL develops in four 1-min stages: (1) diffuse emissions are confined to the bottomside of the $F$ layer at altitudes $h \geq 200 \mathrm{~km}$, (2) a spot-within-ring pattern appears while gradually descending to $\sim 200 \mathrm{~km}$ where the optical ring stops but the central spot still descends, (3) near $\sim 180 \mathrm{~km}$, the spot splits into bright, $\sim 1-2 \mathrm{~km}$ in diameter, filaments rapidly descending at $\sim 300 \mathrm{~m} / \mathrm{s}$ to $\sim 160 \mathrm{~km}$, and (4) the descent slows down between $160 \mathrm{~km}$ and the terminal altitude $h_{g l}^{*} \approx 150 \mathrm{~km}$. Two artificial plasma layers, near $h_{g l}^{*}$ and on the bottomside near $200 \mathrm{~km}$ with the plasma frequency $f_{p e}^{D L}>2.85 \mathrm{MHz}$ and $f_{u h r}=2.85 \mathrm{MHz}$, respectively, are evident in the 
volume emission rate (b) and true height profiles (f).

Field-aligned IL echoes from MUIR (yellow curves in Figure 4.29d) appear during stage 2, disappear during the fast descent, and emerge intensified at the end. This pattern is similar to the MUIR signals in Figures 4.24 and 4.27. The blue-line intensity (c) decreases from $\sim 10 \mathrm{R}$ (phase 2 and 3) to $\sim 5 \mathrm{R}$ at the end indicating the decrease of the ionizing population. Near the terminal $h_{g l}^{*} \approx h_{r e}^{*}$ the central bright emissions somewhat quench themselves, while retreating in altitude until the end of the transmission. During the underdense, continuous onperiod, the artificial plasma near $h_{g l}^{*} \quad$ is quenched several times, initiating the whole process over again from higher altitudes until the UH resonance ceases. We note that the BRIOCHE experiment with $\mathrm{MZ}$ injections at 4.1 and $4.2 \mathrm{MHz}\left(<3 f_{c e}\right)$ on 2 September 2011 also shows this pattern. Namely, after the descending PL echoes stop near $h_{r e}^{*} \sim 195 \mathrm{~km}$, the layer is
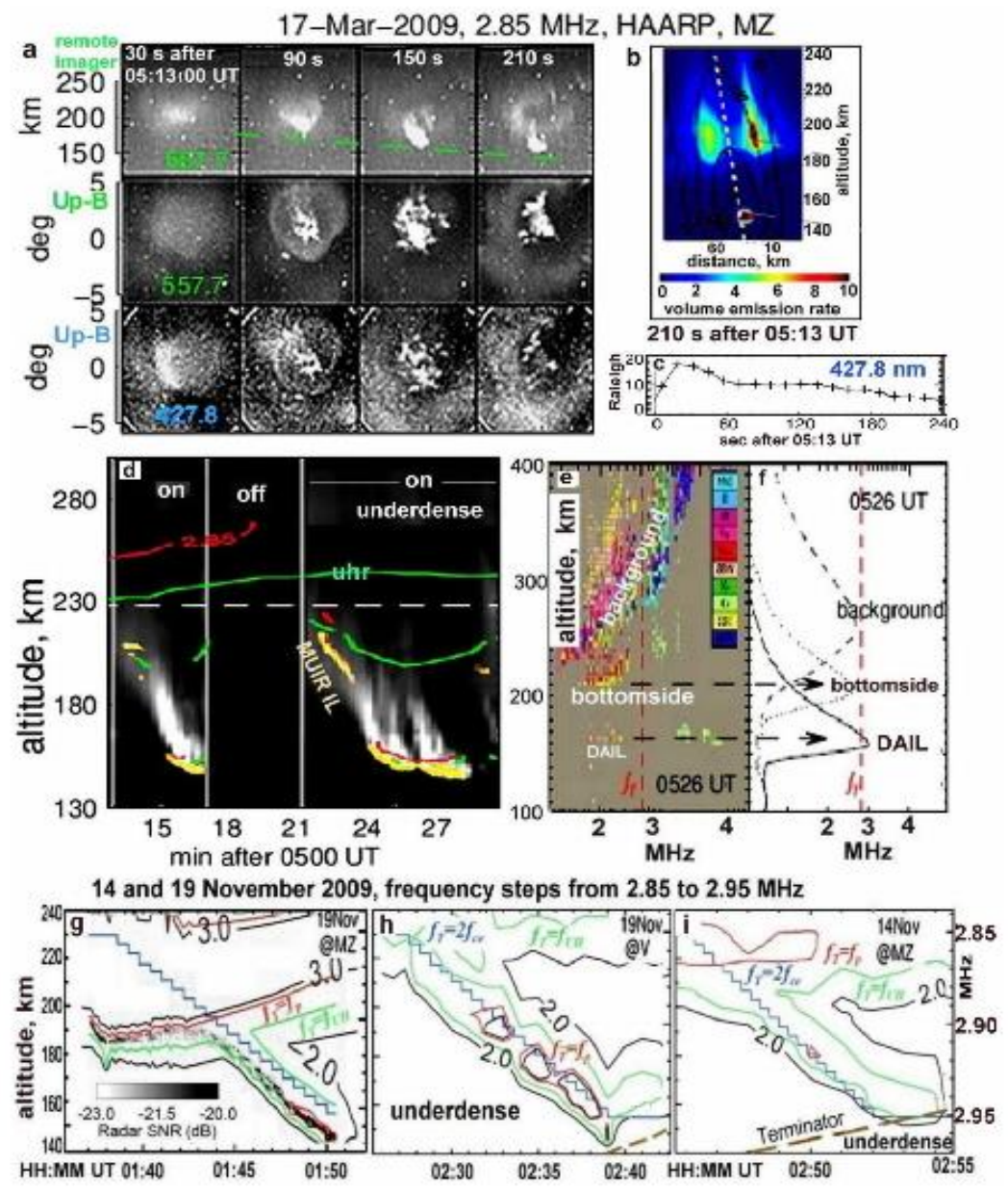

Figure 4.29. (a) Images of optical emissions from (top) the remote site at $557.7 \mathrm{~nm}$, with altitudes indicated, and from the HAARP site at ( $2^{\text {nd }}$ row) $557.7 \mathrm{~nm}$ and ( $3^{\text {rd }}$ row) $427.8 \mathrm{~nm}$. (b) A tomographic cross-section of the volume emission rate with the HAARP magnetic field line superimposed. (c) Average calibrated intensities at $427.8 \mathrm{~nm}$ for the central spot. (d) Altitudetime plot of 557.7-nm emissions from a remote imager with the MUIR ionline backscatter power (in yellow) superimposed (the white dashed line indicate $h_{g r}$ ). (e) Ionogram taken at 05:26 UT with background F-region echoes and two lower layers near 160 and $200 \mathrm{~km}$ virtual height. (f) True height profiles inverted from the 05:26 UT ionogram. (bottom) True height profiles for HF pointing at (g) MZ and (h) vertical on 19-Nov-2009 and (i) $\mathrm{MZ}$ on 14-Nov-2009. Contours of plasma frequency for 2.0 and $3.0 \mathrm{MHz}$ are shown in black, and the matching altitudes $\mathrm{h}_{\mathrm{gr}}(\mathrm{t}), \mathrm{h}_{\mathrm{uh}}(\mathrm{t})$, and $\mathrm{h}_{0}(\mathrm{t})$ are shown in blue, green, and red, respectively. The solar terminator height (brownish dashed lines) and the MUIR ion-line backscatter power from MZ (dark grey) are overlaid. After Pedersen et al. [2010; 2011] and Mishin and Pedersen [2011].

quenched a few times and initiated over again until the end of the transmission.

More details are revealed in the 14 (and 19) November 2009 experiments that started with 1 min transmission at $f_{T}^{0}=2.85 \mathrm{MHz}$, then stepped up by $5 \mathrm{kHz}$, dwelling on each transmitter 
frequency $f_{T}(t)$ for $18 \mathrm{~s}(36 \mathrm{~s})$ to reach $2.95 \mathrm{MHz}$ after $6 \mathrm{~min}(12 \mathrm{~min})$. As a result, the gyroresonance height $h_{g r}\left(f_{T}(t)\right)$ (blue stepwise lines) was decreasing from $h_{g r}^{0} \approx 230 \mathrm{~km}$ to $h_{g r}^{*} \approx 150 \mathrm{~km}$. On 19 November (Figure 4.29g), a $\sim 2 \mathrm{~km}$-thick layer of MUIR field-aligned IL echoes (dark grey) persisted in the overdense ionosphere near $h_{0}^{0} \approx 190 \mathrm{~km}$ until the frequency mismatch $\Delta f_{u h}(t)=2 f_{c e}(t)-f_{u h r}\left(h_{u h}\right)$ decreased to $\sim 10 \mathrm{kHz}$. Then, the UH layer, with the IL backscatter on the top, started to descend while keeping $\Delta f_{u h}(t) \sim 10 \mathrm{kHz}$. Near the terminal $h_{r e}^{*} \approx h_{0}^{*} \approx 145 \mathrm{~km}$, the DL plasma frequency $f_{p e}^{D L}$ exceeded $f_{0}$ and the intense IL echoes persisted until the transmitter turn off at 01:50:30 UT. Similarly, on 14 November (Figure 4.29i), the descent was facilitated as soon as $\Delta f_{u h}$ decreased to $\sim 5 \mathrm{kHz}$. The transition to the underdense background near 02:50 UT $(\approx 180 \mathrm{~km})$ had not impacted the descent. Instead, $f_{p e}^{D L}$ reached $f_{T}(t)$ and remained just below it until the end. The layer persisted near $150 \mathrm{~km}$ with $\Delta f_{u h}^{*} \approx 0$ until the solar terminator crossing, i.e., the sunlit-to-dark transition.

In the initially-underdense $(f o F 2=2.7 \mathrm{MHz})$ ionosphere later on 19 November (Figure $4.29 \mathrm{~h}$ ), $f_{T}^{0} \quad$ was close to $f_{u h r}$, and the contours started to descend at the very beginning. The DL has become overdense much of the time after 02:32 UT, below $200 \mathrm{~km}$, with $\Delta f_{u h} \approx 15 \mathrm{kHz}$ on average. MUIR did not detect any IL echoes until 02:32 UT (no vertical pointing was used). The signals were very weak from all pointing directions (the strongest from $3^{\circ} \mathrm{S}$ ) and not seen in $\mathrm{MZ}$. The layer retreated in altitude and disappeared following the terminator crossing.

\subsubsection{Theory}

\section{SLT and the Magnetic Zenith Effect in Descending Layers}

Overall, descending layers appear at various injection angles but in some experiments only for field-aligned (MZ) HF beam pointing. Numerical simulations [Eliasson et al., 2015] show that the most favorable incidence angles for the SLT development (implying no anomalous absorption) are $3.5^{\circ} \mathrm{S}$ and $10.5^{\circ} \mathrm{S}$. For real 3-D beams, $3.5^{\circ} \mathrm{S}$ and vertical are within the half-power beam width, as are $10.5^{\circ} \mathrm{S}$ and $\mathrm{MZ}$. As the 2-D swelling factor at $\mathrm{MZ}$ increases to about the same as at vertical, the greater SLT extent $l_{L T}$ at MZ makes field-aligned HF beam pointing more efficient for the SLT acceleration. This is consistent with the greater NC power at MZ [Mishin et al., 2016, Figure 5], which persists from the very beginning of injections, alike the $\mathrm{MZ}$ effect in incoherent backscatter at low powers.

For $s \geq 3$, the IL/PL backscatter persists in, and out of, the forbidden band, $\left|f_{0}-s f_{c e}\right| \leq f_{l h r}$. The descent speed slows down after passing the gyroresonance altitude, $h_{g r}$, while MUIR signals intensify. Greater HF powers correspond to greater descent speeds and weaker MUIR signals. For 
$f_{0} \sim 2 f_{c e}$, the descent is facilitated at $2 f_{c e}-f_{u h r} \leq 10-15 \mathrm{kHz}$, while the IL backscatter enhances near the terminal point. When the heating frequency is close to $2 f_{c e}$ at the terminal, the artificial plasma persists until the heater turn off or the terminator crossing. The latter indicates that the artificial ionization is facilitated in the sunlit ionosphere.

Figures 4.26 and 4.27 show that the SLT (the persistent central IL peak and NC) and UH (DM and BUM) features coincide. Mishin et al. [2016] argue that the DL formation is not tied to either the BUM mechanism even at $h>h_{b u m}^{*}>h_{g r}$ (section 3.5) or TPI. Namely, the data show that the descent continues after passing $h_{b u m}^{*}$ and does not depend on the $\mathrm{BUM}_{\mathrm{D}}$ intensity. The DM features with DL (at MZ) or without (at vertical) are similar, as contrasted to the NC overshoot behavior at vertical and persistence at MZ. That is, the DL development follows the NC power. Furthermore, the descending features and DM appear too rapidly for TPI-related striations to develop. Thus, it is conclusive that the fast PPI UH processes (section 3.5) leading to DM and BUM generation do not play a major role during these events and that the DL are mainly related to the SLT development.

The observation that DL appear in the sunlit ionosphere at ERPs as small as $\sim 0.2 \mathrm{GW}$ [Djuth et al., 1994] and that the sunlit-to-dark transition quenches the persistent DL at the terminus indicates more efficient ionization when photoelectrons are present. This is consistent with the SLT acceleration process, which is enhanced in the presence of the ambient suprathermal population [e.g., Mishin et al., 2004]. Another consequence is that the DL speed anticorrelates with the MUIR backscatter power. Actually, as the accelerated population increases, collapse is arrested at larger scales due to the greater absorption by the tail electrons [e.g., Robinson, 1997]. As MUIR detects $\lambda_{\text {res }}=33 \mathrm{~cm}$ waves, with the resonance electron energy $\varepsilon_{\text {res }}<\varepsilon_{\text {ion }}$, the enhanced tail population at $\varepsilon_{\min }>\varepsilon_{\text {res }}$ can absorb/reduce the collapsing energy before cavitons reach $\lambda_{\text {res }}$. In other words, while the density of the ionizing $\left(\varepsilon>\varepsilon_{\text {ion }}\right)$ electrons $n_{t}^{\text {ion }}$ and $v_{D}$ increase, the detectable wave energy reduces and vice versa. At the same time, the NC signature indicates the waves invisible by MUIR. While the SLT overshoot develops, the collapse proceeds to short scales thus making detection possible. This is consistent with the gap between the initial overshoots and the persistent PL/IL signals, which is of the order of the ionization length of accelerated electrons [Mishin and Pedersen, 2011].

The wave energy in the short-scale (acceleration) region can be increased by conversion of long-scale waves on short-scale density oscillations (eq. 24). The presence of such oscillations is indicated by the large altitudinal spread and cloud-like structure of the IL/PL and BF/DVH layers and their broad scattering pattern. The fast disappearance of the DVH layer and the BF recovery to that of period 1 after the end of period 2 [Sergeev et al., 2013] is consistent with decay of smallscale irregularities. In the heated plasma $\left(T_{e} \sim 0.3-0.4 \mathrm{eV}\right)$, short-scale oscillations can be produced 
by both "burned out" cavities in the SLT region and the heat flux-driven instability (section 3.6.1). The latter easily develops at altitudes $\sim 200 \mathrm{~km}$ where $\left|\nabla_{\|} \ln T_{e}\right|^{-1} \leq 100 \mathrm{~km}$ [Dhillon and Robinson, 2005], while the mean free path of heated electrons $\lambda_{T}$ is $\sim 2-3 \mathrm{~km}$. This instability leads to the asymmetry between down- and up-shifted shoulders in the IL spectrum consistent with the observed significant asymmetry between up- and down-shifted ion lines in the IL layers.

\section{Mitigation of Anomalous Absorption}

The SLT-driven ionization model (sections 3.7 and 4.6.1) considers the pump wave continuously reaching the descending plasma resonance altitude, $h_{c}$, which is consistent with the persistent SLT signatures in the course of descent. The latter seems contradictory to the concept of anomalous absorption related to the TPI in the UH layer [e.g., Gurevich et al., 1996], especially when the SLT features coincide with the wellknown UH signatures, such as DM and BUM. The obvious difference between the persistent descent at $6.77 \mathrm{MHz}$ and overshoot at 7.1 $\mathrm{MHz}$ in Figure 4.24 for vertical injections is easily understood [Mishin et al., 2016]. The reason is most evident for the $\mathrm{DL}$ at $6.77 \mathrm{MHz}$ which is within the forbidden band of $5 f_{c e}$ and hence TPI is inhibited.

For $f_{0}=7.1 \mathrm{MHz}, 1-\mathrm{D}$ calculations give $h_{m z}-h_{u h} \sim 3 l_{m z}(\approx 230 \mathrm{~m})$ at $\mathrm{MZ}$ and $\Delta h_{u h} \approx 45 l_{0}\left(\Delta_{0}\left(h_{u h}\right)>>w_{0}\left(h_{u h}\right)\right)$ at vertical

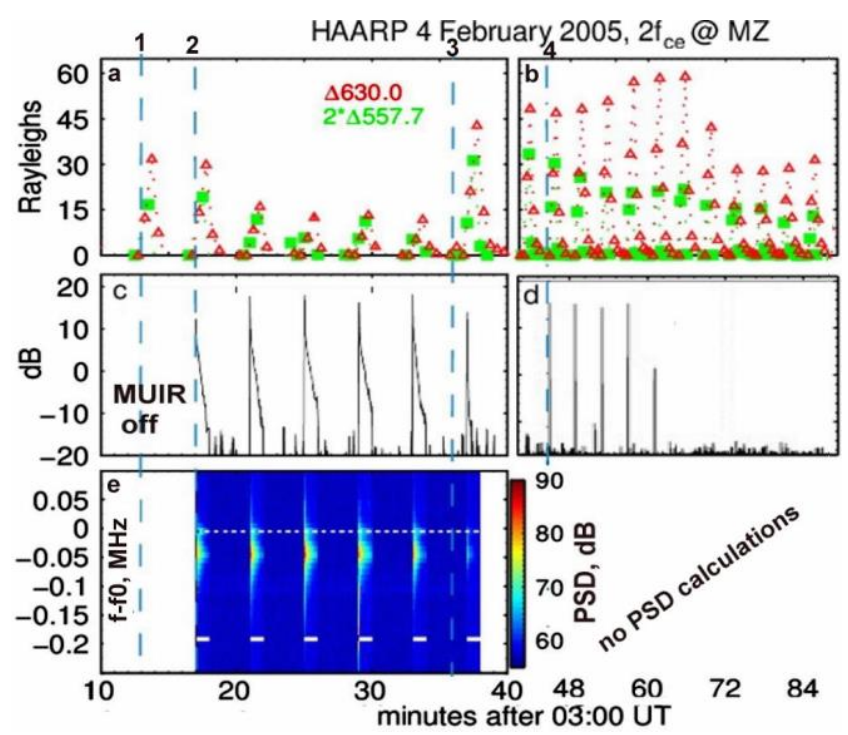

Figure 4.30. A frequency pass through 2 fce on 4 February 2005: (left row) Before and (right) after the double resonance crossing: (a-b) The average backgroundsubtracted 630 (red) and doubled 557.7 (green) nm optical intensity in a $2.5^{\circ}$ cone about the HAARP beam center, (cd) plasma-line backscatter power, and (e) time-frequency profile of the upshifted PL power spectral density plotted with 2-s integrated data. White bars show time intervals for $\mathrm{O}$-mode HF on. The dashed line shows the frequency offset from the UHF diagnostic radar frequency minus the HF pump frequency. The labeled vertical dashed lines indicate the gyroresonance (1-3) and double resonance (4) crossing. After Kosch et al. [2007a] and Oyama et al. [2006].

[Mishin et al., 2016]. That is, the Airy pattern at MZ overlaps the UH layer and the pump field is almost magnetic field-aligned. The ill-placed pump polarization and scattering off short-scale cavitons and ion oscillations hamper TPI/ PPI ${ }_{U H / E B}^{O}$ at MZ. At vertical, however, one has the standard conditions for TPI, consistent with IL overshoots and the artificial radio-aurora enhanced by $\sim 10-20 \mathrm{~dB}$ over that at MZ [Dhillon and Robinson, 2005].

For $f_{0}=5.7-5.9 \mathrm{MHz}$, the difference $h_{m z}-h_{u h}$ at HAARP is $\leq 5 l_{m z}$, while $\Delta h_{u h}$ is $\sim 50 l_{0}$ at vertical [Mishin et al., 2016]. Therefore, the same explanation as for 7.1 MHz is viable. Such 
reasoning, however, fails for the DL in Figures 4.25 and 4.26, with $h_{u h}$ well below $h_{m z}$. In this case, $\mathrm{PPI}_{U H / E B}^{O}$ and PPI ${ }_{U H}$ are suggested to be the cause [Mishin et al., 2016]. Indeed, these fastdeveloping processes lead to spectral transfer toward long scales and off-perpendicular propagation angles (section 3.5.1), thereby inhibiting the resonant instability and hence deep thermal cavitons/striations needed for anomalous absorption.

The persistent IL backscatter in Figure 4.29 shows that TPI is mitigated for $f_{0} \leq 2 f_{c e}$, most likely by virtue of the dispersive properties (section 3.2). Unfortunately, neither spectral IL nor coherent radar backscatter data are available in these experiments to specify the LT regime and FAI, respectively. Some important clues can be deduced from low-power ( $\left.P_{0} \approx 10 \mathrm{MW}\right) \mathrm{HAARP}$ experiments exploring a frequency pass through $2 f_{c e}$ in a decaying ionosphere [Djuth et al., 2005; Kosch et al., 2005; 2007a; Oyama et al., 2006]. On 20 March 2004, the HF beam was pointed in MZ with 4-min (2/2-min on/off) and 10-min (8/2-min on/off) cycles during 06:00-07:00 UT when $f_{0}=2.75 \mathrm{MHz}$ was less than $2 f_{c e}\left(h_{m z}\right)$ [Djuth et al., 2005]. Enhanced $777.4 \mathrm{~nm}$ emissions indicated accelerated electrons at $\geq 10.7 \mathrm{eV}$, while the SEE spectrum [Djuth et al., 2005, Figure 4] featured the DM family and NC, alike that in Figures 4.26 and 4.27.

Figure 4.30 shows the salient features of artificial aurora and MUIR PL echoes during the Kosch et al. [2007a] experiment on 4 February 2005, with 1-min on/off MZ injections at $2.85 \mathrm{MHz}$ (see figure caption). Clearly, 630.0 and $557.7 \mathrm{~nm}$ emissions increase near the gyroresonance $h_{0} \approx h_{g r}$ [cf. Mishin et al., 2005a] but little change is seen after the double resonance crossing ( $h_{u h} \approx h_{g r}$ ) at about 03:45 UT. However, the Kodiak SuperDARN radar backscatter increased by 10-15 dB after the crossing [Kosch et al., 2007a]. Actually, this transition starts when $f_{0}$ exceeds $2 f_{c e}\left(h_{u h}\right)$ by $\sim 6 \mathrm{kHz}$, i.e., at the forbidden band boundary. The crossing particularly affected fieldaligned PL backscatter measured by MUIR with the range and time resolution of $1.5 \mathrm{~km}$ and 10 $\mathrm{ms}$, respectively. Namely, persistent backscatter before the crossing changed to $\leq 2$-s overshoots thereafter. Broad SLT peaks with the purely-growing mode at $f_{0}$ and the WT decay and first cascade lines in persistent PL spectra indicate the co-existence of the WT and SLT regimes. This experiment unambiguously shows that the TPI at MZ is inhibited for $f_{u h r} \leq 2 f_{c e}$ but developed for $f_{u h r}>2 f_{c e}$.

\section{Langmuir Turbulence in the UH Layer}

Mishin et al. [2016] explain the presence of two descending IL layers at $f_{0}=4.5 \mathrm{MHz}$ (Figure 4.26A) by the concurrent excitation of SLT near the plasma resonance and $h_{u h}$ (via OTSI Lo section 3.5.2). Indeed, the height difference $h_{m z}-h_{u h} \approx 4 \mathrm{~km}$ (at the scale height $l_{n}=50 \mathrm{~km}$ ) is close to the observed one at $20 \%$ and $50 \%$ of full power and at the beginning of descent at full 
power. The weaker signal in the lower layer is consistent with the weaker wave energy in the UH layer. Let us assume that two temperature profiles, with peaks near $h_{m z}$ and $h_{u h}$, overlap in such a way that the temperature peak is formed between the layers. Then, the asymmetry in the Dopplershifted spectra in each layer can be understood in terms of propagation of short-scale, $\lambda<\lambda_{\text {res }}$, ion acoustic waves along $\nabla_{\|} T_{e}$. Though in this case the heat flux instability is not necessary, it can also contribute to the asymmetry and broadening of the layers.

As OTSI ${ }_{L o}^{U H}$ is facilitated at $f_{0}>s f_{c e}(s \geq 3)$, the SLT acceleration in the UH layer is supposed to be greater above the gyroresonance than below. In addition, Vlasov simulations of electron acceleration by $2 \mathrm{~V} / \mathrm{m}$ O-mode waves in the upper hybrid layer [Najmi et al., 2016; 2017] show that the evolution of the transverse electron distribution for $f_{o}$ below and above $4 f_{c e}$ drastically differ. Namely, stochastic bulk heating occurs at $f_{o}<4 f_{c e}$ and acceleration of suprathermal tail electrons otherwise. Both processes favor the emergence of DL and faster descent speeds at $f_{0}>4 f_{c e}$ [Sergeev et al., 2013], as well as generation of $427.8 \mathrm{~nm}$ emissions [Gustavsson et al., 2006].

The experiments with $f_{0} / f_{c e} \geq 3$ reveal a broad SEE peak downshifted from the pump by 0.3-0.5 kHz [Leyser, 2001; Mahmoudian et al., 2013], which can be understood in terms of the IAPD instability (section 3.5). Samimi et al.'s [2014] numerical simulations at $\omega_{u h r}<2 \omega_{c e}$ and $T_{e} \geq 3 T_{i}$ show that the IAPD instability in the $\mathrm{UH}$ layer results in collapsing cavitons and concomitant (parallel $\mathbf{B}_{0}$ ) electron acceleration, resembling the SLT process. In addition to OTSI ${ }_{L o}^{U H}$, this process can contribute to the DL in Figure 4.29 rapidly developing in the underdense ionosphere, where $\mathrm{PPI}_{L}^{O}$ is unlikely until the DL plasma becomes dense enough $\left(f_{p e}^{(D L)} \rightarrow f_{0}\right)$. Besides, at small $\left|2 f_{c e} / f_{0}-1\right|$ cyclotron acceleration [e.g., Dimant et al., 1992; Kuo, 2015] can contribute to the DL formation, as with intensified optical emissions at $h_{0} \rightarrow h_{g r}$ for low powers in Figuree 4.29.

It is relevant to note that the $\mathrm{PPI}_{U H}$ decay of the IAPD-excited primary UH waves can generate LH waves. In turn, lower hybrid nonlinear coupling can lead to LH collapse that creates elongated density (LH) cavitons [e.g., Musher et al., 1978; Shapiro and Shevchenko, 1984]. Kosch et al. [2007a] interpreted the DM and weak backscatter from the Kodiak radar at $f_{0}<2 f_{c e}$ by conversion of the secondary UH waves and scattering of the radar beam on LH cavitons, respectively.

Finally, Mishin and Pedersen [2011] suggested the thermal self-focusing instability near the plasma resonance to be the cause of km-scale bright filaments in the Pedersen et al. [2010] experiment (Figure 4.29a-f), as in Kosch et al. [2007b] for low powers. According to theory [e.g., 
Guzdar et al., 1998], km-scale filaments grow initially and in $\sim 10 \mathrm{~s}$ break into smaller (10s-100s meters) scale sizes. During descent, the plasma resonance descends by several kilometers in $\sim 10$ $\mathrm{s}$, thereby precluding breaking into small scales. However, in the persistent layer at the terminus $h_{D}^{*}$ small-scale irregularities can fully develop for $f_{0}$ well under $2 f_{c e}\left(h_{D}^{*}\right)$ and scatter the HF beam, thereby quenching the SLT-related ionization and thus the DL. As soon as the layer decays and irregularities fade out, the artificial plasma can be created again, resembling the quenching and reappearance of the DL in Figure 4.29d. However, the instability is suppressed when $f_{0}$ tends

to $s f_{c e}$ [e.g., Mjølhus, 1993; Starodubtsev et al., 2007], in agreement with the DL's persistence at $h_{D}^{*}$ in November 2009.

In conclusion, the overall data show that the SLT acceleration is the principal cause of the DL formation, though the contribution of the UH/EB processes is also important.

\subsection{Other Active Experiments}

\subsubsection{Artificial Ionospheric Horizontal Periodic Irregularities (APIs)}

Leyser and Wong [2009] reviewed ways that powerful HF waves can provide information about the geospace environment as well as ways that they can influence the environment in a very broad sense. One of these ways is to use such HF facilities to create artificial periodic irregularities (API). This sophisticated technique was pioneered at the SURA facility [Belikovich et al., 2002; Belikovich et al., 2007] and used regularly there since 1986 for studies of the upper atmosphere and ionosphere. The periodicity of the irregularities is due to standing wave formation between the incident radiowave, sent from the heater, and the reflected wave from the F region or E region. This standing wave causes small deviations in heating of the ionospheric plasma at $\lambda=2$ intervals due to the reflected wave interfering with the upgoing wave. This effectively creates a Braggscattering structure, as the refractive index of the plasma is also modified at $\lambda=2$ intervals. In one version of the technique applicable when the irregularities have a sufficiently long lifetime, the heating is turned off and the ionosphere is probed by short radar pulses. The probing pulses do not necessarily have to use the same frequency or polarization. A sufficient condition for observing echoes with enhanced power is that the wavelength of the probing pulse matches the periodic structure created by the heating pulse. There are several mechanisms that create irregularities in the refractive index of ionospheric plasma, depending on which region they are created. Due to the wide range of ionospheric effects involved with the formation of the irregularities, variations of the API method can be used to study various parameters of the ionosphere between the lower D region and the specular reflection altitude of the pump wave. Some of the parameters measured are vertical velocity, neutral density, electron and ion-temperature and electron density. API has been implemented at Arecibo, EISCAT, HIPAS and HAARP in short campaigns. New results from this technique with the upgraded EISCAT heater are presented in Vierinen et al. [2013] where one of the heater antenna arrays is used as the receiving antenna. 


\subsubsection{E Region Ionospheric Perturbations}

Effects of HF heating on the E region have been seen in VHF coherent radar backscatter by the STARE radars [Hibberd et al., 1983; Hoeg et al., 1986; Hysell et al., 2010] and attributed to a resonance instability. Hoeg [1986] found some experimental evidence and theoretical arguments that the horizontal drift directions of the artificial meter scale irregularities were rotated up to $60^{\circ}$ compared to the natural flow direction, which were postulated to result from a polarization electric field in the modified conductivity of the artificial striations in the heater region. Although Hoeg [1986] modelled the small scale striation drift to be influenced by polarization fields set up due to the modified conductivity within them, the same should apply on the larger scale of the heated volume as proposed by Stubbe and Kopka [1977]. Detailed 3D measurements of the modified E region by a more sensitive radar (like EISCAT_3D) than those presently available should be made to test these models. The postulated horizontal polarization electric field and eventual field-aligned currents which provide the ionosphere-magnetosphere coupling should be measured, as a crucial component in understanding the magnetosphere-ionosphere coupling effects mentioned in section 4.5.

$\mathrm{HF}$ enhanced ion and plasma lines have also been observed in the E region at Arecibo and EISCAT [Rietveld et al. 2002 and references therein; Schlatter et al. 2013], similar to those seen in the $\mathrm{F}$ region. Bulk electron temperature and density changes were not observed in these experiments however.

The combined effect of HF heating and naturally occurring Farley-Bueneman instability on the E region temperature under varying auroral electrojet conditions were examined theoretically [Robinson, 1994] and experimentally using the EISCAT UHF radar by Robinson et al. [1995, 1998]. Electron temperature increases in the Hall current region were measured but under strong electrojet conditions there were indications of weaker RF-induced heating Robinson et al. [1995] than under weak electrojet conditions. In a later experiment there were indications that during RFheating the temperature of the E region was lower than when the heater was off. This "cooling" effect of the HF wave may appear surprising, but can be explained by a heater-induced reduction in the amplitude of Farly-Bueneman waves. The results were tentative and warrant repeating with an incoherent scatter radar that is faster at measuring the $\mathrm{E}$ region, which will be possible with the EISCAT_3D radar which is under construction.

\subsubsection{Region and Mesospheric Perturbations}

D region perturbations of the collision frequency by HF heating is an ever-present effect which is the basis for ELF/ELF modulation of conductivity and currents in the lower ionosphere as discussed in section 4.5. This region is known as the mesosphere to atmospheric scientists and is a difficult region to probe since it borders on space, being too high for balloons. Artificial electron heating in the $\mathrm{D}$ region has been detected indirectly by various methods. These include MF partial reflection [Holt et al., 1985], perturbations on signals from ground VLF transmitters [Barr et al., 1985b], incoherent scatter radar [Kero et al., 2000], MF cross modulation [Senior et al., 2010], the 
effect on cosmic noise absorption (CNA) [Senior et al., 2011 and references therein]. In several of these studies the modelled temperature increase is larger by a factor of about 1.5-2.0 [Senior et $a l ., 2011]$ than that derived from the measurements. Possible reasons for the discrepancy are that the assumption that the electron distribution remains Maxwellian during heating of the plasma, which is implicit in the models is a potential source of error. Another reason could be that the radiated power from the HF facility is overestimated. This power is calculated assuming a perfectly conducting ground, but modelling using typical ground conductivities and dielectric constants based on measurements suggests that only about $75 \%$ of this power is actually radiated. This problem is an important one and needs resolving if such experiments are to provide better quantitative parameters of the lower ionosphere/upper atmosphere.

Mesospheric investigations with the EISCAT HF facility have been vigorously pursued ever since the discovery that electron temperature increases in the D region can weaken [Chilson et al. 2000] and strengthen [Havnes et al., 2003] Polar Mesospheric Summer Echoes (PMSE). Modelling [e.g. Mahmoudian et al., 2011] predicts that the relative importance of charging of mesospheric dust versus electron diffusion on the echo strength increases with the radar wavelength, and recent work has concentrated on using up to four different wavelength radars to compare the measured suppression and overshoot phenomena with these predictions [e.g. Senior et al., 2014]. Modelling the experimentally measured characteristic overshoot curves of the PMSE strength in response to the HF pulses can potentially provide information on the aerosol particle sizes, a crucial parameter in understanding the mesosphere and in explaining the PMSE phenomenon.

Artificial modulation of Polar Mesospheric Winter Echoes (PMWE) by ionospheric heating has been reported by Kavanagh et al. [2006]. The effect on PMWE of ionospheric heating illustrates similar gross features to those for PMSE: a sharp decrease in power when HF heating is switched on followed by a sharp increase when heating is switched off. A recovery of the PMWE was identified during 10 second heating suggestive of ionized dust playing a role in PMWE formation. 


\section{Conclusions}

Active experiments employing powerful HF transmitters have revealed a wealth of information on many different physical phenomena. These experiments can initiate and sustain a diverse set of concurrent phenomena. These phenomena include "ionospheric" processes, such as generation of the ionospheric plasma instabilities and irregularities; generation of ELF waves propagating in the ionosphere and in the Earth-ionosphere wave guide. They also include "magnetospheric" processes, such as generation of ULF/ELF waves propagating into the magnetosphere; interactions between these waves and particles in the magnetosphere; particle precipitation into the ionosphere; and secondary ionospheric phenomena caused by this precipitation.

Due to the strong electrodynamic coupling between the ionosphere and the magnetosphere, particularly in the ULF frequency range, both magnetospheric and ionospheric geophysical processes may contribute to the results of active experiments observed in space and on the ground. The complex nature and simultaneous generation of different instabilities responsible for weak and strong turbulence in the heated volume makes it hard to identify conclusively a single specific mechanism responsible for all the effects observed in the experiments, particularly when these experiments are conducted with very powerful HF waves ( ERP > $200 \mathrm{MW}$ ).

In addition, not only the latitude of the heating facility, but power, frequency and the polarisation of high power EM wave can activate different mechanisms which may lead to very different effects observed in space and on the ground. For example, excitation of ULF/ELF/VLF waves or FAIs illustrate different behaviour under $\mathrm{O}$ and $\mathrm{X}$ mode pump waves. Another example is the luminous structures/spots in the ionosphere that can be generated by local ionospheric processes or by the magnetospheric electrons precipitating in the ionosphere by ULF/ELF waves generated by the pump wave.

The goal of this review is to describe the current state of understanding in the field and identify directions in ionosphere-magnetosphere space research where active experiments are indispensable.

\section{The Current State of Knowlege}

We can conclude that currently we do have a good quantitative understanding of basic physics of generation of ULF/ELF/VLF waves propagating into the magnetosphere and into the Earthionosphere waveguide with HF heating of the ionosphere. Numerous experimental and theoretical studies reveal correlation between the amplitude of these waves and parameters of the ionosphere (in particular, the strength of the electrojet/electric field in the ionosphere and the plasma density in the ionsopheric D, E, and F regions) and pump wave for different generating mechanisms.

We also understand quite well, both in theory and experiment, various types of plasma instabilities produced by the ionosphere heating with relatively low-power HF waves (ERP <200 MW). These classical instabilities, described in section 3, heat the plasma, produce waves, accelerate particles and generate magnetic field-aligned irregularities in plasma density with spatial sizes and temporal dynamics well described by the current theory and confirmed by the 
observations.

\section{Outstanding issues for future research:}

1. Detailed investigation into what happens when the ionosphere is heated with very high-power (ERP > $200 \mathrm{MW}$ ) HF waves. In this case several different types of turbulence may happen simultaneously in the same disturbed volume of the ionospohere and the interactions between waves and density disturbances produced by different mechanisms can give significantly different results compared with the linear case, when these mechanisms occur independently from each other.

2. Propose a theoretical mechanism behind the generation of small-scale field aligned irregularities by X-mode heating which has been observed experimentally.

3. Understand generation, spatial distribution and dynamics of super-small-scale irregularities (SSSIs).

4. Investigate sub-beam sized structures (kilometre scale) and their dynamics within the artificial optical emissions.

5. Understand the mechanism behind the unexplained heater-induced field-aligned UHF backscatter (WAILES).

6. Investigate generation of supra-thermal electrons and their energy spectrum for different pump frequencies, in particular the $2^{\text {nd }}$ gyro-harmonic. This investigation is also important to understand the mechanism behind descending ionisation layer.

7. Investigate the temporal development of density perturbations along the ambient magnetic field from the generation/resonance region. In particular, the velocity of the duct formation observed in the experiments is much higher than that predicted by the theory.

8. Investigate propagation of ULF/VLF waves generated in the ionospheric $\mathrm{D}$ and $\mathrm{E}$ regions into the magnetosphere, in particular:

- How do these waves interact with plasma in the magnetosphere and in the conjugate hemisphere?

- Do these waves cause a precipitation of the suprathermal electrons leading to artificial aurora?

- What is the system of the currents (in the ionosphere and along the ambient magnteic field) generated by the ionospheric heating?

- How do these currents interact/drive plasma turbulence in the ionopsphere?

9. Investigate triggering and control of the development of the ionospheric feedback instability. Does this lead to the generation of very intense, small-scale field-aligned currents and density structures in the ionosphere? In other words, to what extent can heating of the ionosphere with a powerful HF transmitters trigger and control development of geomagnetic disturbances such as substorms?

\section{To answer these questions:}


- More active experiments involving HF heating of the ionosphere at HAARP, SURA, EISCAT and Arecibo need to be conducted.

- The heating facilities (HAARP and SURA) need to have a comprehensive set of advanced high-resolution sensors, in particular an incoherent scatter radar. The combined set of diagnostics ionosondes, radars, magnetometers, optical cameras) provides the capablility to create a comprehensive, real-time, multi-dimensional picture of waves and plasma and enables science questions regarding plasma waves and turbulence to be investigated in detail.

- The EISCAT_3D to be operational in 2021 will provide unprecedented time and spatial resolution measurements with an extended coverage in height and horizontal extent of the heated volume. A new HF heater close to the EISCAT_3D site is highly recommended in order to address a many of the listed outstanding science questions that cannot be adequately performed with the location of the existing heater. These science topics include different scale sizes and distribution of FAIs, field aligned phenomena such as WAILES, detailed investigation of plasma wave and turbulence with high temporal and spatial resolution, as well as D-region/mesospheric physics. In addition, the high temporal resolution of EISCAT_3D will enable studies of duct formation and propagation. Low frequency capability ( $2^{\text {nd }}$ gyroharmonic) needs to be incorporated into the EISCAT heater in order to increase the ability to perform heating experiments under wider range of geophysical conditions. The current capability is a limiting factor, particularly during solar minimum [Tsuda et al., 2018]. Heating at the $2^{\text {nd }}$ gyroharmonic is particularly effective in examining artificial ionisation and optical emissions.

- Particularly important for the understanding of ULF/ELF wave experiments and related outstanding questions are observations on satellites and in the locations magnetically conjugate to the heater. There the observations can be performed on stationary or mobile (ships, airplanes, satellites) platforms.

- Special attention needs to be devoted to dedicated satellite missions, like DSX, RESONANCE, and CubeSats, which from the start will be oriented to work in conjunction with ground transmitters and will have approprite sensors and trajectories to obtain in-situ measurements of plasma parameters not accessible by ground-based diagnostics In particular in-situ measurements of electron and ion temperature and density distributions are extremely important to understand the electron acceleration mechanisms associated with various plasma instabilities generated by the action of high power HF waves.

- Finally, comprehensive, time-dependent, multi-dimensional numerical models describing propagation of ULF/ELF/VLF waves in the highly coupled and complex magnetosphereionosphere system need to be developed. These models should be comprehensive enough $\underline{t o}$ predict the anticipated results from the experiments, so that they can to be used for planning future experiments. Numerical simulations are also important for the understanding and interpretation of the obtained results. 


\section{Acknowledgements}

We acknowledge fruitful discussion of active experiments and heating facilities with $\mathrm{H}$. C. Carlson, M. Cohen, M. Golkowski, S. Grach, M. M. Mogilevsky, E. Nossa, K. D. Papadopoulos, T. Pedersen, B. Watkins.

This work was made possible by the ISSI funding of the working group "Past, Present and Future of Active Experiments in Space" and supported in part through CNES grant DEMETER 2874949; Air Force Research Laboratory contract FA95550-17-D-0001; Air Force Office of Scientific Research; Russian Education Ministry project 3.1844.2017. 


\section{References}

Akbari, H., A. Bhatt, C. La Hoz, and J. Semeter (2017), Incoherent scatter plasma lines: Observations and applications, Space Sci. Rev., doi:10.1007/s11214-017-0355-7.

Allen E.M., Thome G.D., Rao P.B. (1974), HF phased array observations of heater-induced spread-F, Radio Sci., 9(11), 905-916.

Andreeva E.S., Frolov V.L., Kunitsyn V.E., Kryukovskii A.S., Lukin D.S., Nazarenko M.O., Padokhin A.M. (2016), Radiotomography and HF ray tracing of the artificially disturbed ionosphere above the SURA heating facility, Radio Sci., 51(6), 638-644, doi:10.1002/2015RS005939.

Ashrafi, M., M.J. Kosch, F. Honary (2006), Heater-Induced altitude descent of the EISCAT UHF ion line enhancements: Observations and modelling, Adv. Space Res., 38, 11, 2645-2652.

Ashrafi M., M. J. Kosch, K. Kaila, and B. Isham (2007), Spatiotemporal evolution of radio wave pumpinduced ionospheric phenomena near the fourth electron gyroharmonic. J. Geophys. Res., 112, A05314, 10.1029/2006JA011938

Atkinson, G. (1970), Auroral arcs: Result of the interaction of a dynamic magnetosphere with the ionosphere, J. Geophys. Res., 75, 4746.

Bailey, V. A. (1937), Interaction by resonance of radio waves, Nature, 139, 68-69.

Bahcivan, H., J. W. Cutler, J. C. Springmann, R. Doe, and M. J. Nicolls (2014), Magnetic aspect sensitivity of high-latitude E region irregularities measured by the RAX-2 CubeSat, J. Geophys. Res., 119(2), 1233-1249.

Bakhmet'eva, N.V., V.V. Belikovich, L. M.Kagan, A. A.Ponyatov, A. V.Tolmacheva, M. C. Kelley, and M. J.Nicolls (2005), New results of studying the lower ionosphere by the method of resonance scattering of radio waves from artificial periodic inhomogeneities, Radiophys. Quantum Elect., 48(9), 673-685.

Barr, R., M. T. Rietveld, H. Kopka, P. Stubbe, and E. Nielsen (1985a), Extra-low-frequency radiation from the polar electrojet antenna, Nature, 317, 6033, 155-157.

Barr, R., M. T. Rietveld, P. Stubbe, and H. Kopka (1985b), The diffraction of VLF radio Waves by a patch of ionosphere illuminated by a powerful HF transmitter, J. Geophys. Res., 90 (A3), 2861-2875.

Barr, R., P. Stubbe, M. T. Rietveld, and H. Kopka (1986), ELF and VLF signals radiated by the 'Polar Electrojet Antenna': Experimental results, J. Geophys. Res., 91, A4, 4451-4459.

Barr, R., M. T. Rietveld, P. Stubbe, and H. Kopka (1988), Ionospheric heater beam scanning: A realistic model of this mobile source of ELF/VLF radiation, Radio Sci., 23 (3), 379-388.

Barr, R. (1996), VLF wave generation using VLF heating and the cubic nonlinearity of the ionosphere, Geophys. Res. Lett. 23, 2165, doi:10.1029/96GL02024.

Barr, R., and P. Stubbe (1997), ELF and VLF wave generation by HF heating: A comparison of AM and CW techniques, J. Atmos. Terr. Phys., 59 (18), 2265-2279.

Barr R., P. Stubbe M. T. Rietveld (1999), ELF wave generation in the ionosphere using pulse modulated HF heating: initial tests of a technique for increasing ELF wave generation efficiency, Ann. Geophys., 17, 759-769.

Belenov A.F., Bubnov V.A., Erukhimov L.M., Kiselev Yu.V., Komrakov G.P., Mityakova E.E., Rubtsov L.N., Uryadov V.P., Frolov V.L., Chugunov Yu.V., Yukhmatov B.V. (1977), On parameters of artificial small-scale ionospheric irregularities, Radiophys. Quant. Electron., 20, 1240-1245. 
Belikovich, V.V., Benediktov E.A., Getmantsev G.G., Erukhimov L.M., Zuikov N.A., Komrakov G.P., Korobkov Yu.S., Mityakov N.A., Rapoport V.O., Trakhtengertz V.Yu., Frolov V.L. (1975), New results of investigations of nonlinear phenomena in the ionosphere, Radiophys. Quant. Electron., 18(4), 377-383.

Belikovich, V.V., Erukhimov L.M., Zyuzin V.A., Korobkov Yu.S., Maksimenko O.I., Nasyrov A.M., Sergeev E.N., Frolov V.L., Shavin P.B. (1988), Times of development and relaxation of artificial smallscale irregularities, Radiophys. Quant. Electron., 31, 181-185.

Belikovich, V. V., S. M. Grach, A. N. Karashtin, D. S. Kotik, and Yu. V. Tokarev (2007), The SURA facility: Study of the atmosphere and space, Radiophys. Quant. Electronics, 50 (7), 497-526.

Belyaev, P., S. Polyakov, V. Rappoport, and V. Trakhtengerts (1990), The ionospheric Alfvén resonator, J. Atmos. Terr. Phys., 52, 781.

Berezin I.V., Boiko G.N., Volkov V.M., Zyuzin V.A., Komrakov G.P., Leonov A.M., Maresov A.N., Ryzhov V.A., Solynin V.A. (1987), Peculiarities of the development and saturation of artificial ionospheric turbulence during a high-power disturbing transitions. Radiophys. Quant. Electron., 30(6), 522-529.

Bernhardt, P., C. Tepley, and L. Duncan (1989), Airglow enhancements associated with plasma cavities formed during ionospheric heating experiments, J. Geophys. Res., 94, 9071.

Bernhardt P.A., Selcher C.A., Lehmberg R.H., et al. (2010), Stimulated Brillouin scatter in magnetized ionospheric plasma, Phys. Rev. Lett., 104, 165004, doi:10.1103/PhysRevLett.104.165004.

Bernhardt P.A., Selcher C.A., Kowtha S. (2011), Electron and ion Bernstein waves excited in the ionosphere by high power EM waves at the second harmonic of the electron cyclotron frequency, Geophys. Res. Let., 38, L19107, doi:10.1029/2011GL049390.

Bernhardt, P., C. Siefring, S. Briczinski, and R. Michell (2016), Large ionospheric disturbances produced by the HAARP HF Facility, Radio Science, 51, 1081-1093, doi:10.1002/2015RS005883.

Blagoveshchenskaya, N. F., V. A. Kornienko, T. D. Borisova, B. Thidé, M. J. Kosch, M. T. Rietveld, E. V. Mishin, R. Y. Luk'yanova, O. A. Troschichev (2001), Ionospheric HF pump wave triggering of local auroral activation, J. Geophys. Res., 106, A12, 29071-29090.

Blagoveshchenskaya, N. F., V. A. Kornienko, T. D. Borisova, M. T. Rietveld, T. Bösinger, B. Thidé, T. B. Leyser, and A. Brekke (2006), Heater-induced phenomena in a coupled ionosphere-magnetosphere system, Adv. Space Res., 38, 2495-2502, doi:10.1016/j.asr.2004.12.047.

Blagoveshchenskaya, N. F., T. D. Borisova, V. A. Kornienko, M. T. Rietveld, T. K. Yeoman, D. M. Wright, M. Rother, H. Lühr, E. V. Mishin, C. Roth, V. L. Frolov, M. Parot, J. L. Rauch (2011), Modification of the high-latitude ionosphere by high-power HF radio waves. 2. Results of coordinated satellite and ground-based observations, Radiophys. Quant. Electronics, 54, 89-101, 0033-8443/11/5402-0089.

Blagoveshchenskaya, N. F., T. D. Borisova, T. K. Yeoman, M. T. Rietveld, I. Häggström, I. M. Ivanova (2013), Plasma modifications induced by an X-mode HF heater wave in the high latitude F region of the ionosphere, J. Atmos. Sol.-Terr. Physics., 105-106, 231-244.

Blagoveshchenskaya, N. F., T. D. Borisova, M. Kosch, T. Sergienko, U. Brändström, T. K. Yeoman, I. Häggström (2014), Optical and Ionospheric Phenomena at EISCAT under Continuous X-mode HF Pumping, J. Geophys. Res., 119, doi:10.1002/2014JA020658.

Blagoveshchenskaya, N. F., T. D. Borisova, T. K. Yeoman, I. Häggström, A. S. Kalishin (2015), Modification of the high latitude ionosphere $\mathrm{F}$ region by $\mathrm{X}$-mode powerful HF radiowaves: 
Experimental results from multi-instrument diagnostics, J. Atmos. Sol.-Terr. Phys., 135, 5063, doi:10.1016/j.jastp.2015.10.009.

Blagoveshchenskaya, N. F., T. D. Borisova, A. S. Kalishin, T. K. Yeoman, I. Häggström (2017), First observations of electron gyro-harmonic effects under X-mode HF pumping the high latitude ionospheric F-region, J. Atmos. Sol.-Terr. Phys., 155, 36-49.

Boiko G.N., Erukhimov L.M., Frolov V.L. (1990), Excitation of small-scale irregularities near the pump wave reflection level, Geomag. Aeronom., 30(6), 843-846.

Borisov, N., A. Gurevich, and K. Papadopoulos (1996), Direct Cerenkov excitation of waveguide modes by a mobile ionospheric heater, Radio Sci., 33(4), 859.

Borisova, T. D., N. F. Blagoveshchenskaya, T. K. Yeoman, and I. Häggström (2017), Excitation of artificial ionospheric turbulence in the high-latitude ionospheric F region as a function of the EISCAT/heating effective radiated power, Radiophys. Quantum Electron., 60, 4, doi: 10.1007/s11141-017-9798-7.

Bösinger, T., A. Kero, P. Pollari, A. Pashin, P. Belyaev, M. Rietveld, T. Turunen, and J. Kangas (2000), Generation of artificial magnetic pulsations in the Pc1 frequency range by periodic heating of the Earth's ionosphere: indications of Alfven resonator effects, J. Atmos. Sol.-Terr. Physics., 62 (4), 277297.

Bryers, C. J., M. J. Kosch, A. Senior, M. T. Rietveld and T. K. Yeoman (2013a), The threshold of plasma instabilities pumped by high frequency radio waves at EISCAT, J. Geophys. Res., 118, 7472-7481, doi:10.1002/2013JA019429.

Bryers, C. J., M. J. Kosch, A. Senior, M. T. Rietveld and W. Singer (2013b), A comparison between resonant and non-resonant heating at EISCAT, J. Geophys. Res., 118, 6766-6776, doi:10.1002/jgra.50605.

Burinskaya, T., and A. Volokitin (1981), Nonlinear dynamics of thermal modulational instability, Physica $2 D, 117-125$.

Bychenkov, V., V. Silin, and S. Uryupin (1988), Ion-acoustic turbulence and anomalous transport, Phys. Rep., 164, 119-215.

Cannon, P. and F. Honary (2015), A GPU-accelerated finite-difference time-domain scheme for electromagnetic wave interaction with plasma, IEEE Trans. Antennas Prop., 63(7), 3042-3054, doi:10.1109/TAP.2015.2423710.

Cannon, P. F. Honary, and N. Borisov (2016), Two-dimensional numerical simulation of O-mode to Zmode conversion in the ionosphere, J. Geophys. Res: Space Phys., 121(3), 2755-2782, doi:10.1002/2015JA022105.

Carlson, H., V. Wickwar, and G. Mantas (1982), Observations of fluxes of suprathermal electrons accelerated by HF excited Langmuir instabilities, J. Atm. Terr. Phys., 12, 1089.

Carlson, H. C. (1993), High power HF modification-geophysics, span of EM effects, and energy budget, Adv. Space Res., 13, 1015â€"1024, doi:10.1016/0273-1177(93)90046-E.

Carlson, H., F. Djuth, and L. Zhang (2017), Creating space plasma from the ground, J. Geophys. Res. Space Physics, 122, 978, doi:10.1002/2016JA023380.

Cheung, P. Y., M. P. Sulzer, D. F. DuBois, D. A. Russell (2001), High-power high-frequency-induced Langmuir turbulence in the smooth ionosphere at Arecibo. II. Low duty cycle, altitude-resolved, observations, Phys. Plasmas, 8(3), 802. 
Chernogor L.F. and Frolov V.L. (2013), Features of the wave disturbances in the ionosphere during periodic heating of the plasma by the SURA radiation, Radiophys. Quantum Electron., 56(5), 276-289.

Chernyshov, A.A., et al. (2016), Approaches to studying the multiscale ionospheric structure using nanosatellites, Geomag. Aeron, 56(1), 72-79, doi:10.1134/S0016793216010047.

Chilson, P. B., E. Belova, M. T. Rietveld, S. Kirkwood, U.-P. Hoppe, First artificially induced modulation of PMSE using the EISCAT heating facility, Geophys. Res. Lett., 27 (23), 3801-3804 (2000)

Cohen, R. and Whitehead J.D. (1970), Radio reflectivity of artificial modification of the ionospheric F layer. Geophys. Res., 75(31), 6439-6445.

Cohen, M.B, M. Golkowski and U.S. Inan (2008), Orientation of the HAARP ELF ionospheric dipole and the auroral electrojet, Geophys. Res. Lett., 35, L02806, doi:10.1029/2007GL032424.

Cohen M. B., U. S. Inan, M. A. Golkowski, and M. J. McCarrick (2010), ELF/VLF wave generation via ionospheric HF heating: Experimental comparison of amplitude modulation, beam painting, and geometric modulation, J. Geophys. Res., 115, A02302, doi:10.1029/2009JA014410.

Cohen M. B., U. S. Inan, D. Piddyachiy, N. G. Lehtinen, M. Golkowski (2011), Magnetospheric injection of ELF/VLF waves with steerable HF heating of the lower ionosphere, J. Geophys. Res., 116, A06308, doi:10.1029/2010JA016194.

Cohen, M., M. Golkowski, N. Lehtinen, U. Inan, and M. McCarrick (2012), HF beam parameters in ELF/VLF wave generation via modulated heating of the ionosphere, J. Geophys. Res., 117, doi:10.1029/2012JA017585.

Cohen, M. B., and U. S. Inan (2012), Terrestrial VLF transmitter injection into the magnetosphere, $J$. Geophys. Res., 117, A08310, doi:10.1029/2012JA017992.

Cohen M. B., R. C. Moore, M. Golkowski, N. G. Lehtinen (2012), ELF/VLF wave generation from the beating of two HF ionospheric heating sources, J. Geophys. Res., 117, A12310, doi:10.1029/2012JA018140.

Cussac, T., Clair, M.A., Ultré-Guerard, P., Buisson, F., Lassalle-Balier, G., Ledu, M., Elisabelar, C., Passot, X., Rey, N. (2006), The DEMETER microsatellite and ground segment, Planet. Space Sci., 54 (5), 413427.

Das A.C., Fejer J.A. (1979), Resonance instability of small-scale field-aligned irregularities, J. Geophys. Res., A84, (11), 6701.

Demekhov A. G., V. Y. Trakhtengerts, M. M. Mogilevsky, L. M. Zelenyi (2003), Current problems in studies of magnetospheric cyclotron masers and new space project "RESONANCE", Adv. Space Res., 32 (3), 355-374

Dhillon, R. S., and T. R. Robinson (2005), Observations of time dependence and aspect sensitivity of regions of enhanced UHF backscatter associated with RF heating, Ann. Geophys., 23, 75.

Dimant, Y., A. Gurevich, and K. Zybin (1992), Acceleration of electrons under the action of intense radiowaves near electron cyclotron harmonics, J. Atm. Terr. Phys., 54, 425-436.

Djuth, F. and D. DuBois (2015), Temporal development of HF-excited Langmuir and ion turbulence at Arecibo, Earth Moon Planets, 116, 19-53, doi:10.1007/s11038-015-9458-x.

Djuth, F., P. Stubbe, M. Sulzer, H. Kohl, M. Rietveld, and J. Elder (1994), Altitude characteristics of plasma turbulence excited with the Tromsø superheater, J. Geophys. Res., 99, 333-339.

Djuth, F. T., B. Isham, M. T. Rietveld, T. Hagfors, C. La Hoz (2004), First 100 ms of HF modification at Tromsø, Norway, J. Geophys. Res., 109, A11307, doi:10.1029/2003JA010236. 
Djuth, F., T. Pedersen, E. Gerken, P. Bernhardt, C. Selcher, W. Bristow, and M. Kosch (2005), Ionospheric modification at twice the electron cyclotron frequency, Phys. Rev. Lett., 94, 125001-1-4, doi: 10.1103/PhysRevLett.94.125001.

DuBois, D.F., D.A. Russell, P.Y. Cheung, M.P. Sulzer (2001), High-power high-frequency-induced Langmuir turbulence in the smooth ionosphere at Arecibo. I. Theoretical predictions for altituderesolved plasma line radar spectra. Phys. Plasmas, 8(3), 791-801.

Dysthe, K., E. Mjølhus, H. Pécseli, and K. Rypdal (1982), Thermal cavitons, Physica Scripta, 1982, T2B, 548-559.

Eliasson, B., C.-L. Chang, and K. Papadopoulos (2012a), Generation of ELF and ULF electromagnetic waves by modulated heating of the ionospheric F2 region, J. Geophys. Res., 117, A10320, doi:10.1029/2012JA017935.

Eliasson, B., X. Shao, G. Milikh, E. V. Mishin, and K. Papadopoulos (2012b), Numerical modeling of artificial ionospheric layers driven by high-power HF-heating, J. Geophys. Res., 117, A10321, doi:10.1029/2012JA018105.

Eliasson, B. (2013), Full-scale simulations of ionospheric Langmuir turbulence, Mod. Phys. Lett. B, 27(8), 1330005, doi:10.1142/S0217984913300056.

Eliasson, B., and K. Papadopoulos (2015), Numerical study of anomalous absorption of O mode waves on magnetic field-aligned striations, Geophys. Res. Lett., 42, 2603--2611, doi:10.1002/2015GL063751.

Eliasson, B., G. Milikh, X. Shao, E. Mishin, and K. Papadopoulos (2015), Incidence angle dependence of Langmuir turbulence and artificial ionospheric layers driven by high-power HF-heating, J. Plasma Phys., 81, 415810201, doi:10.1017/S0022377814000968.

Erukhimov L.M., Metelev S.A., Mityakov N.A., Frolov V.L. (1978), Hysteresis phenomenon by artificial excitation of irregularities in ionospheric plasma, Radiophys. Quant. Electron., Engl. Transl., 21, 12091211.

Erukhimov, L.M., Metelev S.A., Mityakov N.A., Frolov V.L., (1983), Initial stage of interaction of intense radio waves with the plasma in the upper ionosphere, Geomag. Aeron., 23(3), 352-356.

Erukhimov, L.M., Metelev S.A., Myasnikov E.N., Mityakov N.A., Frolov V.L., (1987), Artificial ionospheric turbulence, Radiophys. Quant. Electron., 30, 156-171.

Erukhimov, L.M., Metelev C.A., Razumov D.V. (1988), Diagnostics of ionospheric irregularities due to artificial radio radiation, Radiophys. Quant. Electron., 31, 928-935.

Farley, D. T., C. La Hoz. B. Fejer (1983), Studies of the self-focusing instability at Arecibo, J. Geophys. Res., 88 (A3), 2093-2102.

Fejer, J. A. (1979), Ionospheric modification and parametric instabilities, Rev. Geophys. Space Phys., 17, $135-153$.

Fejer, J. A., H. M. Ierkic, R. F. Woodman, J. Rottger, M. Sulzer, R. A. Behnke, and A. Veldhuis (1983), Observations of the HF-enhanced plasma line with a 46.8-MHz radar and reinterpretation of previous observations with the 430-MHz radar, J. Geophys. Res., 88 (A3), 2083-2092.

Fish, C.S., et al. (2014), Design, development, implementation, and on-orbit performance of the Dynamic Ionosphere CubeSat Experiment mission, Space Sci. Rev., 181, 61-120, doi:10.1007/s11214-0140034-x. 
Fennelly, J. (2009), Demonstration and Science Experiment (DSX) Space Weather Experiment (SWx), Solar Physics and Space Weather Instrumentation III, eds. S. Fineschi, J. Fennelly, Proc. of SPIE v. 7438, 743805-1-10, doi:10.1117/12.828321.

Forslund, D. (1970), Instabilities associated with heat conduction in the solar wind and their consequences, J. Geophys. Res., 75, 17-28, doi:10.1029/JA075i001p00017.

Frolov V.L. (1981), Influence of modulation transfer in the action of a powerful emission on the ionospheric plasma, Radiophys. Quant. Electron., 24, 354-356.

Frolov V.L., Erukhimov L.M., Metelev S.A., Sergeev E.N. (1997), Temporal behavior of artificial smallscale ionospheric irregularities: Review of experimental results, J. Atmos. Solar-Terr. Phys., 59(18), 2317-2333.

Frolov, V.L., Ermakova E.N., Kagan L.M., et al. (2000), Features of the broad upshifted structure in stimulated electromagnetic emission spectra, J. Geophys. Res., 105 (A9), 20,919-20,933.

Frolov, V.L., Sergeev E.N., Ermakova E.N., et al. (2001), Spectral features of stimulated electromagnetic emissions, measured in the 4.3-9.5 MHz pump wave frequency range, Geophys. Res. Lett., 28 (16), 3103-3106.

Frolov V.L. (2003), Control of spectral characteristics of artificial low-frequency ionosphere turbulence, Inter. J. Geomag. Aeron., 4(2), 159-165.

Frolov, V.L., Sergeev E.N., Komrakov G.P., et al. (2004), The ponderomotive narrow continuum $\left(\mathrm{NC}_{\mathrm{p}}\right)$ component in stimulated electromagnetic emission spectra, J. Geophys. Res., 109, A07304, doi:10.1029/2001JA005063.

Frolov V.L., et al., (2007), Modification of the terrestrial ionosphere by high-power short-wave radio radiation, Physics. Uspekhi, 50(3), 15-24, doi:10.1070/PU2007v050n03ABEH006282.

Frolov V.L., et al., (2012), Gyroharmonic features of the irregularities HF-induced in the ionosphere, Radiophys. Quant. Electron., 55(6), 357-381.

Frolov V.L., et al., (2014), Generation of artificial ionospheric irregularities in the mid-latitude ionosphere modified by high-power high-frequency X-mode radio waves, Radiophys. Quant. Electron., 57(6), 393416, doi:10.1007/s11141-014-9523-8.

Frolov V.L., Rapoport V.O., Schorokhova E.A., Belov A.S., Parrot M., and Rauch J.-L. (2016), Features of the electromagnetic and plasma disturbances induced at the altitudes of the Earth's outer ionosphere by modification of the ionospheric F2 region using high-power waves radiated by the SURA heating facility, Radiophys. Quant. Electron., 59(3), 177-198, doi:10.1007/s11141-016-9688-4.

Frolov V.L., Bolotin I.A., Vertogradov G.G., and Vertogradov V.G. (2017), Generation of super smallscale artificial ionospheric irregularities in the ionosphere pumped by high-power HF radio waves, Radiophys. Quant. Electron., 60(6), 450-455, doi:10.1007/s11141-017-9813-z.

Galeev, A., R. Sagdeev, V. Shapiro, and V. Shevchenko (1977), Langmuir turbulence and dissipation of high-frequency energy, Sov. Phys. JETP, 46, 711-719.

Galeev, A., and R. Sagdeev (1979), Nonlinear plasma theory, in Reviews of Plasma Phys., v. 7, ed. M.A. Leontovich, p.1-180, Consultants Bureau, New York.

Galeev, A., R. Sagdeev, V. Shapiro, and V. Shevehenko (1983), Beam plasma discharge and suprathermal electron tails, in Active Experiments in Space (Alpbach, Austria), Eur. Space Agency Spec. Publ., ESA SP-195, 151-155.

Gdalevich G.L., Z. Klos, Y.M. Mikhailov (2003), Excitation of ELF-VLF electric fields in ionospheric 
plasma in active experiments (Intercosmos-24 data), Geomag. Aeron., 43(5), 602-609.

Gelinas, L., M. Kelley, M. Sulzer, E. Mishin, and M. Starks (2003), In situ observations during an HF heating experiment at Arecibo: Evidence for Z-mode and electron cyclotron harmonic effects, $J$. Geophys. Res., 108, 1382, doi:10.1029/2003JA009922.

Getmantsev, G., N. Zuikov, D. Kotik, L. Mironenko, N. Mityakov, V. Rapoport, Y. Sazonov, V. Trakhtengerts, and V. Eidman (1974), Combination frequencies in the interaction between high-power short-wave radiation and ionospheric plasma, JETP Lett., 20, 101.

Getmantsev, G. G., A. V. Gul'el'mi, B. I. Klain, D. S. Kotik, S. M. Krylov, N. A. Mityakov, V. O. Rapoport, V. Yu. Trakhtengerts, and V. A. Troitskaya (1977), Excitation of magnetic pulsations under the action on the ionosphere of radiation of a powerful short-wave transmitter, Radiophys. Quantum Electron., 20, 703-705, doi:10.1007/BF01040635.

Gigliotti, A., W. Gekelman, P. Pribyl, S. Vincena, A. Karavaev, X. Shao, A. S. Sharma, and D. Papadopoulos (2009), Generation of polarized shear Alfven waves by rotating magnetic field source, Phys. Plasmas, 16, 092106.

Gołkowski, M., U.S. Inan, A.R. Gibby, M.B. Cohen (2008), Magnetospheric amplification and emission triggering by ELF/VLF waves injected by the 3.6 MW HAARP ionospheric heater, J. Geophys. Res., 113, A10201, doi:10.1029/2008JA013157.

Gołkowski, M., U.S. Inan, M.B. Cohen (2009), Cross modulation of whistler mode and HF waves above the HAARP ionospheric heater, Geophys. Res. Lett, 36, L15103, doi:10.1029/2009GL039669.

Gołkowski, M., U.S. Inan, M.B. Cohen, A.R. Gibby (2010), Amplitude and phase of nonlinear magnetospheric wave growth excited by the HAARP HF heater, J. Geophys. Res., 115, A00F04, doi:10.1029/2009JA014610.

Gołkowski, M., M. B. Cohen, D. L. Carpenter, and U. S. Inan (2011), On the occurrence of ground observations of ELF/VLF magnetospheric amplification induced by the HAARP facility, J. Geophys. Res., 116, A04208, doi:10.1029/2010JA016261.

Gordon W.E., Carlson H.C., Showen R.L. (1971), Ionospheric heating at Arecibo: First tests, J. Geophys. Rev., 76(31), 7808-7813.

Grach, S.M., Karashtin, N.A., Mityakov, N.A., et al. (1977), Parametric interaction between electromagnetic radiation and the ionospheric plasma, Radiophys. Quant. Electron., 20, 1254-1258.

Grach, S. (1979), Thermal parametric instability in ionospheric plasma at frequencies close to $\omega_{\mathrm{He}}$ and $2 \omega_{\mathrm{He}}$, Radiophys. Quantum Electron., 22, 357-361.

Grach, S., N. Mityakov, V. Rapoport, and V. Trakhtengertz (1981), Thermal parametric turbulence in a plasma, Physica D, 2, 102-106.

Grach, S.M., Thidé B., Leyser T.B. (1994), Plasma waves near the double resonance layer in the ionosphere, Radiophys. Quant. Electron., 37, 392-402.

Grach, S., S. Sergeev, V. Yashnov, and V. Kotov (2008), Spectra of stimulated electromagnetic emission of the ionosphere during pump frequency sweeping near gyroharmonics. II. Discussion of the results, Radiophys. Quantum Electron., 51, $499-514$.

Grach, S., E. Sergeev, E. Mishin, A. Shindin, and M. McCarrick (2015), Intermediate downshifted maximum of stimulated electromagnetic emission at high-power HF heating: A new twist on an old problem, J. Geophys. Res. Space Phys., 120, 666--674, doi:10.1002/2014JA020423.

Grach, S., E. Sergeev, E. Mishin, and A. Shindin (2016), Dynamic properties of ionospheric plasma 
turbulence driven by high-power high-frequency radiowaves, Physics-Uspekhi, 59, 1091-1128, doi: 10.3367/UFNe.2016.07.03786.

Gurevich, A. (1978), Nonlinear Phenomena in the Ionosphere, Springer-Verlag, New York.

Gurevich A.V., Lukyanov A.V., Zybin K.P. (1995), Stationary state of isolated striations developed during ionospheric modification, Phys. Lett. A. 206, 247-259.

Gurevich, A., A. Lukyanov, and K. Zybin (1996), Anomalous absorption of powerful radio waves on the striations developed during ionospheric modification, Phys. Lett. A, 211, 363-372.

Gurevich A., Hagfors T., Carlson H., Karashtin, A., Zybin, K. (1998), Self oscillations and bunching of striations in ionospheric modifications, Phys. Lett. A. 239, 385-392.

Gurevich, A., K. Zybin, H. Carlson, and T. Pedersen (2002), Magnetic zenith effect in ionospheric modifications, Phys. Lett. A, 305, 264-274.

Gurevich A.V., Zybin K.P. (2006), Strong field aligned scattering of UHF radio waves in ionospheric modification, Phys. Lett. A, 358, 159-165.

Gurevich, A. (2007), Nonlinear effects in the ionosphere, Physics - Uspekhi, 50, 1091-1121, doi: 10.1070/PU2007v050n11ABEH006212.

Gustavsson B., T. Sergienko, M.J. Kosch, M.T. Rietvelilild, A. Steen, B.U.E. Brandstrom, T.B. Leyser, B. Isham, P. Gallop, T. Aso, M. Ejiri, K. Kaila, J. Jussila and H. Holma (2005), The electron distribution during HF pumping - a picture painted in all colours, Ann. Geophys., 23, 1747-1754.

Gustavsson, B., T. Leyser, M. Kosch, M. Rietveld, Å. Steen, B. Brändström, and T. Aso (2006), Electron gyroharmonic effects in ionization and electron acceleration during High-Frequency pumping in the ionosphere, Phys. Rev. Lett., 97, 195002-1-4, doi: 10.1103/PhysRevLett.97.195002.

Gustavsson, B., M. Kosch, A. Wong, T. Pedersen, C. Heinselman, C. Mutiso, B. Bristow, J. Hughes, and W. Wang (2008), First estimates of volume distribution of HF-pump enhanced emissions at 6300 and 5577A ${ }^{\circ}$ : A comparison between observations and theory, Ann. Geophys., 26, 3999-4012, 2008a.

Gustavsson, B., and B. Eliasson (2008), HF radio wave acceleration of ionospheric electrons: Analysis of HF-induced optical enhancements, J. Geophys. Res., 113, A08319, doi:10.1029/2007JA012913, 2008 b.

Gustavsson, B., R. Newsome, T. B. Leyser, M. J. Kosch, L. Norin, M. McCarrick, T. Pedersen, and B. J. Watkins (2009), First observations of X-mode suppression of O-mode HF enhancements at $6300 \mathrm{~A}$, Geophys. Res. Lett., 36, L20102, doi:10.1029/2009GL039421.

Gustavsson, B., M. T. Rietveld, N. V. Ivchenko, and M. J. Kosch (2010), The Rise and Fall of Electron Temperatures I: Ohmic Heating of Ionospheric Electrons from Under-Dense HF-radio Wave Pumping, J. Geophys. Res., 115, A12332, doi:10.1029/2010JA015873.

Guzdar, P., P. Chaturvedi, K. Papadopoulos, and S. Ossakow (1998), The thermal self-focussing instability near the critical surface in the high-latitude ionosphere, J. Geophys. Res., 103, 2231-2237.

Havnes, O., C. La Hoz, L. I. Naesheim, M. T. Rietveld (2003), First observations of the PMSE overshoot effect and its use for investigating the conditions in the summer mesosphere, Geophys Res. Lett., 30 (23), 2229, doi:10.1029/2003GL018429.

Helliwell, R. (1965), Whistlers and Related Ionospheric Phenomena, Stanford University Press, Stanford.

Helliwell, R. (1988), VLF simulated experiments in the magnetosphere from Siple station, Antarctica, Rev. Geophys., 26, 551.

Hibberd, F. H., E. Nielsen, P. Stubbe, H. Kopka, and M. T. Rietveld (1983), Production of auroral zone E region irregularities by powerful HF heating, J. Geophys. Res., 88(A8), 6347-6351. 
Hoeg, P. (1986), Directional changes in the irregularity drift during artificial generation of striations, Phys. Scr., 33, 469-474.

Holma H., K. Kaila, M.J. Kosch and M.T. Rietveld (2006), Recognising the blue emission in artificial aurora, Adv. Space Res., 38, 11, 2653-2658.

Holt, O., A. Brekke, T. Hansen, H. Kopka, and P. Stubbe (1985), HF modification of the auroral D-region detected by a partial reflection experiment, J. Atmos. Terr. Phys., 47 (6), 537-545.

Honary, F., T. Robinson, D. Wright, A. Stocker, M. Rietveld, and I. McCrea (1999), First direct evidence of the reduced striations at pump frequencies close to the electron gyroharmonics, Ann. Geophys., 17, 1235--1238.

Honary, F., N. Borisov, M. Beharrell, and A. Senior (2011), Temporal development of the magnetic zenith effect, J. Geophys. Res., 116, A06309, doi:10.1029/2010JA016029.

Hosseini, P., M. Gołkowski, and D. L. Turner (2017), Unique concurrent observations of whistler mode hiss, chorus, and triggered emissions, J. Geophys. Res., 122, doi:10.1002/2017JA024072.

Huang, J., and S. Kuo (1994), A theoretical model for the broad upshifted maximum in the stimulated electromagnetic emission spectrum, J. Geophys. Res., 99, 19,569-19,576.

Huang, J., and S. P. Kuo (1995), A generation mechanism for the downshifted peak in stimulated electromagnetic emission spectrum, J. Geophys. Res. 100, 21,433-21,438, doi:10.1029/95JA02302.

Hussein, A., W. Scales, and J. Huang (1998), Theoretical and simulation studies of broad up-shifted sideband generation in ionospheric stimulated radiation, Geophys. Res. Lett., 25, 955-958.

Hysell, D. L., M. C. Kelley, Y. M. Yampolski, V. S. Beley, A. V. Koloskov, P. V. Ponomarenko, and O. F. Tyrnov (1996), HF radar observations of decaying artificial field-aligned irregularities, J. Geophys. Res., 101(A12), 26981-26993, doi:10.1029/96JA02647.

Hysell, D. L., E. Nossa, and M. McCarrick (2010), Excitation threshold and gyroharmonic suppression of artificial E region field-aligned plasma density irregularities, Radio Sci., 45, RS6003, doi:10.1029/2010RS004360.

Hysell, D., R. Miceli, E. Kendall, N. Schlatter, R. Varney, B. Watkins, T. Pedersen, P. Bernhardt, and J. Huba (2014), Heater-induced ionization inferred from spectrometric airglow measurements, $J$. Geophys. Res., 119, doi:10.1002/2013JA019663.

Inan, U., H. Chang, R. Helliwell, W. Imhof, J. Reagan, and M. Walt (1985), Precipitation of radiation belt electrons by man-made waves: A comparison between theory and measurement, J. Geophys. Res., 90, 359.

Inan, U., T. Bell, J. Bortnik, and J. Albert (2003), Controlled precipitation of radiation belt electrons, $J$. Geophys. Res., 108, 1186, doi:10.1029/2002JA009580.

Inan, U.S., M. Gołkowski, D. L. Carpenter, N. Reddell, R.C. Moore, T.F. Bell, E. Paschal, P. Kossey, E. Kennedy, S.Z. Meth, (2004) Multi-hop whistler-mode ELF/VLF signals and triggered emissions excited by the HAARP HF heater, Geophys. Res. Lett. 31, L24805, doi:10.1029/2004GL021647.

Isham, B., C. Hoz, M. Rietveld, T. Hagfors, and T. Leyser (1999a), Cavitating Langmuir turbulence observed during EISCAT high latitude ionospheric interaction experiments, Phys. Rev. Lett. 83, 2576.

Isham, B., T. Hagfors, E. Mishin, M. Rietveld, C. LaHoz, W. Kofman, and T. Leyser (1999b), A search for the location of the HF excitation of enhanced ion acoustic and Langmuir waves with EISCAT and the Tromsø heater, Radiophys. Quantum Electron., 42, 607-618. 
Isham, B., C. A. Tepley, M. P. Sulzer, Q. H. Zhou, M. C. Kelley, J. S. Friedman, and S. A. Gonz'alez (2000), Upper atmospheric observations at the Arecibo Observatory: Examples obtained using new capabilities, J. Geophys. Res., 105, 18609-18637, doi:10.1029/1999JA900315.

Istomin, Y., and T. Leyser (1995), Parametric decay of an electromagnetic wave near electron cyclotron harmonics, Phys. Plasmas, 2, 2084-2097.

James, H. G., V. L. Frolov, E. S. Andreeva, A. M. Padokhin, and C. L. Siefring (2017), SURA heating facility transmissions to the CASSIOPE/e-POP satellite, Radio Sci., 52, 259-270, doi:10.1002/2016RS006190.

Jin, G., M. Spasojevic, and U. Inan (2009), Relationship between electrojet current strength and ELF signal intensity in modulated heating experiments, J. Geophys. Res., 114, 8301, doi:10.1029/2009JA014122.

Jones T.B., Robinson T., Stubbe P., Kopka H. (1983), A hysteresis effect in the generation of field-aligned irregularities by a high-power radio wave, Radio Sci., 18(6), 835-839.

Kagan, L.M., M. C. Kelley, F. Garcia et al. (2000), The structure of electromagnetic wave-induced 557.7$\mathrm{nm}$ emission associated with a aporadic-E event over Arecibo, Phys. Rev. Lett., 85(1), 218-221.

Kagan L.M., et al. (2006), Optical and RF diagnostics of the ionosphere over the SURA facility: Review of results, Radio Phys. Radio Astron., 11(3), 221-241.

Kalogerakis, K. S., T. G. Slanger, E. A. Kendall, T. R. Pedersen, M. J. Kosch, B. Gustavsson, and M. T. Rietveld (2009), Remote Oxygen Sensing by Ionospheric Excitation (ROSIE), Ann. Geophys., 27, 1-7.

Karavaev, A.V., N.A. Gumerov, K. Papadopoulos, X. Shao, A.S. Sharma, W. Gekelman, A. Gigliotti, P. Pribyl and S. Vincena (2010), Generation of whistler waves by a rotating magnetic field source, Phys. Plasmas, 17, 012102.

Karavaev, A.V., N.A. Gumerov, K. Papadopoulos, X. Shao, A.S. Sharma, W. Gekelman, A. Gligliotti, P. Pribyl and S. Vincena (2011), Generation of shear Alfvén waves by a rotating magnetic field source: 3D simulations, Phys. Plasmas 18, 032113.

Kavanagh, A.J., F. Honary, M. T. Rietveld, and A. Senior (2006), First observations of the artificial modulation of polar mesospheric winter echoes, Geophys. Res. Lett., 33, L19801, doi:10.1029/2006GL027565.

Kelley, M. C., T. L. Arce, J. Salowey, M. Sulzer, W. T. Armstrong, M. Carter, and L. Duncan (1995), Density depletions at the 10-m scale induced by the Arecibo heater, J. Geophys. Res., 100, 17367-17376.

Kelley, M. C., W. E. Swartz, and K. Heki (2017), Apparent ionospheric total electron content variations prior to major earthquakes due to electric fields created by tectonic stresses, J. Geophys. Res., 122, 6689-6695, doi:10.1002/2016JA023601.

Kendall, E., R. Marshall, R. T. Parris, A. Bhatt, A. Coster, T. Pedersen, P Bernhardt, and C. Selcher (2010), Decameter structure in heater-induced airglow at the High frequency Active Auroral Research Program facility, J. Geophys. Res., 115, A08306, doi:10.1029/2009JA015043.

Kero, A., T. Bösinger, P. Pollari, E. Turunen and M. Rietveld (2000), First EISCAT measurement of electron-gas temperature in the artificially heated D-region ionosphere, Ann. Geophys., 18 (9), 12101215.

Kolesnikova, E., T. R. Robinson, J. A. Davies, D. M. Wright, and M. Lester (2002), Excitation of Alfven waves by modulated HF heating of the ionosphere, with application to FAST observations, Ann. Geophys., 20, 57-67.

Kosch, M., T. Rietveld, T. Hagfors and T. Leyser (2000), High-latitude HF-induced airglow displaced 
equatorwards of the pump beam, Geophys. Res. Lett., 27, 2817-2820, doi: 10.1029/2000GL003754.

Kosch, M. J., M. T. Rietveld, A. J. Kavanagh, C. Davis, T. Yeoman, F. Honary and T. Hagfors (2002), High-latitude pump-induced optical emissions for frequencies close to the third electron gyroharmonic, Geophys. Res. Lett., 29, 23, 2112, doi:10.1029/2002GL015744.

Kosch, M., M. Rietveld, A. Senior, I. McCrea, A. Kavanagh, B. Isham, and F. Honary (2004), Novel artificial optical annular structures in the high latitude ionosphere, Geophys. Res. Lett., 31, L12805, doi: 10.1029/2004GL019713.

Kosch, M., T. Pedersen, J. Hughes, R. Marshall, E. Gerken, A. Senior, D. Sentman, M. McCarrick, and F. Djuth (2005), Artificial optical emissions at HAARP for pump frequencies near the third and second gyroharmonic, Ann. Geophys., 23, 1585-1592.

Kosch, M., T. Pedersen, E. Mishin, S. Oyama, J. Hughes, A. Senior, B. Watkins, and B. Bristow (2007a), Coordinated optical and radar observations of ionospheric pumping for a frequency pass through the second electron gyroharmonic at HAARP, J. Geophys. Res., 112, A06325, doi:10.1029/2006JA012146.

Kosch, M., T. Pedersen, E. Mishin, M. Starks, E. Gerken-Kendall, D. Sentman, S. Oyama, and B. Watkins (2007b), Temporal evolution of pump beam self-focusing at the High-frequency Active Auroral Research Program, J. Geophys. Res., 112, A08304, doi:10.1029/2007JA012264.

Kosch, M. J., T. Pedersen, M. T. Rietveld, B. Gustavsson, S. M. Grach and T. Hagfors (2007c), Artificial optical emissions in the high-latitude thermosphere induced by powerful radio waves: An observational review, Adv. Space. Res., 40, 365-376, doi:10.1016/j.asr.2007.02.061.

Kosch, M., Y. Ogawa, M. Rietveld, S. Nozawa, and R. Fujii (2010), An analysis of pump-induced artificial ionospheric ion upwelling at EISCAT, J. Geophys. Res., 115, A12317, doi:10.1029/2010JA015854.

Kosch, M. J., C. Bryers, M. T. Rietveld, T. K. Yeoman and Y. Ogawa (2014a), Aspect angle sensitivity of pump-induced optical emissions at EISCAT, Earth, Planets and Space, 66, 159, doi: 10.1186/s40623014-0159-x.

Kosch, M. J., H. Vickers, Y. Ogawa, A. Senior, N. Blagoveshchenskaya (2014b), First observation of the anomalous electric field in the topside ionosphere by ionospheric modification over EISCAT, Geophys. Res. Lett., 41, 21, 7427-7435, doi:10.1002/2014GL061679.

Kotik, D. S., and E. N. Ermakova (1998), Resonances in the generation of electromagnetic signals due to the thermal cubic nonlinearity in the lower ionosphere, J. Atmos. Sol. Terr. Phys., 60(12), 1257.

Kunitsyn, V. E., E. D. Tereshchenko, E. S. Andreeva, and I. A. Nesterov (2010), Satellite radio probing and radio tomography of the ionosphere, Usp. Fiz. Nauk, 180(5), 548-553, doi:10.3367/UFNr.0180.201005k.0548.

Kunitsyn V.E., Andreeva E.S., Frolov V.L., Komrakov G.P., Nazarenko M.O., Padokhin A.M. (2012), Sounding of HF heating-induced artificial ionospheric disturbances by navigation satellite radio transmissions, Radio Sci., 47(4), RS0L15, doi:10.1029/2011RS004957.

Kuo, S., M. Lee, and P. Kossey (1997), Excitation of oscillating two-stream instability by upper hybrid pump in ionospheric heating experiments at Tromsø, Geophys. Res. Lett., 24, 2969-2972.

Kuo, S. (2003), Parametric excitation of lower hybrid waves by electron plasma waves, Phys. Lett. A, 307, 244-248.

Kuo, S., A. Snyder, P. Kossey, C.-L. Chang, and J. Labenski (2011), VLF wave generation by beating of two HF waves in the ionosphere, Geophys. Res. Lett., 38, L10608, doi:10.1029/2011GL047514.

Kuo, S., A. Snyder, P. Kossey, C.-L. Chang, and J. Labenski (2012), Beating HF waves to generate VLF 
waves in the ionosphere, J. Geophys. Res., 117, A03318, doi:10.1029/2011JA017076.

Kuo, S. (2013), Electron cyclotron harmonic resonances in high-frequency heating of the ionosphere, Phys. Plasmas, 20, 092124-1-8, doi:10.1063/1.4822336.

Kuo, S. (2015), Ionospheric modifications in high frequency heating experiments, Phys. Plasmas, 22, 012901-1-16, doi:10.1063/1.4905519.

Lehtinen, N. and U. Inan (2008), Radiation of ELF/VLF waves by harmonically varying currents into a stratified ionosphere with application to radiation by a modulated electrojet, J. Geophys. Res., 113, A06301, doi:10.1029/2007JA012911.

Leyser, T. B., B. Thidé, H. Derblom, Å. Hedberg, B. Lundborg, P. Stubbe and H. Kopka (1989), Stimulated electromagnetic emission near electron cyclotron harmonics in the ionosphere, Phys. Rev. Lett., 63, 11, 1145-1147.

Leyser, T.B., Thide B., Derblom H., et al. (1990), Dependence of stimulated electromagnetic emission on the ionosphere and pump wave, J. Geophys. Res., 95 (A10), 17,233-17,244.

Leyser, T.B., Thide B., Waldenvik M., et al. (1993), Spectral structure of stimulated electromagnetic emission between electron cyclotron harmonics, J. Geophys. Res., 98 (A10), 17,597-17,606.

Leyser, T.B., Thide B., Waldenvik M., et al. (1994), Downshifted maximum features in stimulated electromagnetic emission spectra, J. Geophys. Res., 99 (A10), 19,555-19,568.

Leyser, T. B., B. Gustavsson, B. U. E. Brandstrom, Å. Steen, F. Honary, M. T. Rietveld, T. Aso and M. Ejiri (2000), Simultaneous measurements of high-frequency pump-enhanced airglow and ionospheric temperatures at auroral latitudes, Adv. Polar Upper Atmos. Res., 14, 1-11.

Leyser, T.B. (2001), Stimulated electromagnetic emissions by high-frequency electromagnetic pumping of the ionospheric plasma, Space Sci. Rev., 98, 223, doi:10.1023/A:1013875603938.

Leyser, T. B., and A. Y. Wong (2009), Powerful electromagnetic waves for active environmental research in geospace, Rev. Geophys., 47, 1, doi:10.1029/2007rg000235.

Mahmoudian, A. W. A. Scales, M. J. Kosch, A. Senior, and M. Rietveld (2011), Dusty space plasma diagnosis using temporal behavior of polar mesospheric summer echoes during active modification, Ann. Geophys., 29, 2169-2179.

Mahmoudian, A., W. Scales, P. Bernhardt, A. Samimi, E. Kendall, J. Ruohoniemi, B. Isham, O. VegaCancel, and M. Bordikar (2013), Ion gyro-harmonic structuring in the stimulated radiation spectrum and optical emissions during electron gyro-harmonic heating, J. Geophys. Res., 118, 1270--1287, doi:10.1002/jgra.50167.

Majeed, T., and D. J. Strickland (1997), New survey of electron impact cross sections for photoelectron and auroral electron energy loss calculations, J. Phys. Chem. Ref. Data, 26, 335349.

Markov, G. A., (2010), Excitation of a magnetospheric maser through modification of the Earth's ionosphere by high-power HF radio emission from a ground-based transmitter, J. Exp. Theor. Phys., 111, 916, doi:10.1134/S1063776110120046.

Mathews, J. D. (2013), A short history of geophysical radar at Arecibo Observatory, Hist. Geo Space Sci., 4, 19-33, doi:10.5194/hgss-4-19-2013.

Maxworth, A. S., M. Gołkowski, M. B. Cohen, R. C. Moore, H. T. Chorsi, S. D. Gedney, and R. Jacobs (2015), Multistation observations of the azimuth, polarization, and frequency dependence of ELF/VLF waves generated by electrojet modulation, Radio Sci., 50, doi:10.1002/2015RS005683. 
McCarrick, M. J., D. Sentman, A. Wong, R. Wuerker, and B. Chouinard (1990), Excitation of ELF waves in the Schumann resonance range by modulated HF heating of the polar electrojet, Radio Sci., 25, 1291.

McCrea, I., A. Aikio, L. Alfonsi, E. Belova, S. Buchert, M. Clilverd, N. Engler, B. Gustavsson, C. Heinselman, Johan Kero, M. Kosch, H. Lamy, T. Leyser, Y. Ogawa, K. Oksavik, A. PellinenWannberg, F. Pitout, M. Rapp, I. Stanislawska and J. Vierinen, The science case for the EISCAT_3D radar (2015), Progress Earth Planet. Sci., 2, 21, doi: 10.1186/s40645-015-0051-8.

Milikh, G. M., and K. Papadopoulos (2007), Enhanced ionospheric ELF/VLF generation efficiency by multiple timescale modulated heating, Geophys. Res. Lett., 34, L20804, doi:10.1029/2007GL031518.

Milikh, G. M., K. Papadopoulos, H. Shroff, C. L. Chang, T. Wallace, E. V. Mishin, M. Parrot, and J. J. Berthelier (2008a), Formation of artificial ionospheric ducts, Geophys. Res. Lett., 35, L17104, doi:10.1029/2008GL034630.

Milikh, G., A. Gurevich, K. Zybin, and J. Secan (2008b), Perturbations of GPS signals by the ionospheric irregularities generated due to HF-heating at triple of electron gyrofrequency, Geophys. Res. Lett., 35, L22102, doi:10.1029/2008GL035527.

Milikh, G. M., E. Mishin, I. Galkin, A. Vartanyan, C. Roth, and B. W. Reinisch (2010a), Ion outflows and artificial ducts in the topside ionosphere at HAARP, Geophys. Res. Lett., 37, L18102, doi:10.1029/2010GL044636.

Milikh, G. M., A. G. Demekhov, K. Papadopoulos, A. Vartanyan, J. D. Huba, and G. Joyce (2010b), Model for artificial ionospheric duct formation due to HF heating, Geophys. Res. Lett., 37, L07803, doi:10.1029/2010GL042684.

Minkoff J., Kugelman P., Weissman I. (1974), Radio frequency scattering from a heated ionospheric volume, 1, VHF/UHF field-aligned and plasma-line backscatter measurements, Radio Sci., 9(11), 941955.

Minkoff J., and Kreppel R. (1976), Spectral analysis and step response of radio frequency scattering from a heated ionospheric volume, J. Geophys. Res., 81(16), 2844-2856.

Mishin, E. (1974), Heat transport in the solar wind, Astrophys. Space Sci., 27, 367-382.

Mishin, E., and V. Telegin (1986), Spectrum of suprathermal electrons in the auroral plasma, Sov. J. Plasma Phys., 12, 509-511.

Mishin, E., T. Hagfors, and B. Isham (2001), A generation mechanism for topside enhanced incoherent backscatter during high frequency modification experiments in Troms $\varnothing$, Geophys. Res. Lett., 28, 479482.

Mishin, E., W. Burke, and T. Pedersen (2004), On the onset of HF-induced airglow at magnetic zenith, $J$. Geophys. Res., 109, A02305, doi: 10.1029/2003JA010205.

Mishin, E., W. Burke, and T. Pedersen (2005a), HF-induced airglow at magnetic zenith: Theoretical considerations, Ann. Geophys., 23, 47-53.

Mishin, E., M. Kosch, T. Pedersen, and W. Burke (2005b), HF-induced airglow at magnetic zenith: Coexistence of the thermal and parametric instabilities near electron gyroharmonics, Geophys. Res. Lett., 32, L23106, doi:10.1029/2005GL023864.

Mishin, E., and T. Pedersen (2011), Ionizing wave via high-power HF acceleration, Geophys. Res. Lett., 38, L01105, doi:10.1029/2010GL046045.

Mishin, E., E. Sutton, G. Milikh, I. Galkin, C. Roth, and M. Förster (2012), F2-region atmospheric gravity waves due to high-power HF heating and subauroral polarization streams, Geophys. Res. Lett., 39, 
L11101, doi:10.1029/2012GL052004.

Mishin, E., B. Watkins, N. Lehtinen, B. Eliasson, T. Pedersen, and S. Grach (2016), Artificial ionospheric layers driven by high-frequency radiowaves: An assessment, J. Geophys. Res. Space Phys., 121, 34973524, doi:1002/2015JA021823.

Mjølhus, E. (1990), On linear conversion in a magnetized plasma, Radio Sci., 25, 1321-1339.

Mjølhus, E. (1993), On the small scale striation effect in ionospheric radio modification experiments near harmonics of the electron gyro frequency, J. Atmos. Terr. Phys., 55, 907.

Mjølhus, E. Helmersen, and D. DuBois (2003), Geometric aspects of HF driven Langmuir turbulence in the ionosphere, Nonl. Proc. Geophys., 10, 151-177.

Mogilevsky, M. M., Zelenyi, L. M., Demekhov, A. G., Petrukovich, A. A. and Shklyar, D. R. (2012) RESONANCE project for studies of wave-particle interactions in the inner magnetosphere, in dynamics of the Earth's radiation belts and inner magnetosphere (eds D. Summers, I. R. Mann, D. N. Baker and M. Schulz), American Geophysical Union, Washington, D.C., doi:10.1029/2012GM001334

Moore R. C., S. Fujjimaru, M. Cohen, M. Golkowski, M. J. McCarrick (2012), On the altitude of the ELF/VLF source region generated during "beat wave" HF heating experiments, Geophys. Res. Lett., 39, L18101, doi:10.1029.2012GL053210.

Moore, R. C, S. Fujimaru, D. A. Kotovsky, and M. Gołkowski (2013), Observations of ionospheric ELF and VLF wave generation by excitation of the thermal cubic nonlinearity, Phys. Rev. Lett., 111 (23), 235007, doi:10.1103/PhysRevLett.111.235007.

Musher, S. L., A. M. Rubenchik and B. I. Sturman (1978), Collective effects associated with lower hybrid heating of plasma, Plasma Phys., 20, 1131-1150.

Mutiso, C., J. Hughes, G. Sivjee, T. Pedersen, B. Gustavsson, and M. Kosch (2008), Previously unreported optical emissions generated during ionospheric heating, Geophys. Res. Lett., 35, L14103, doi:10.1029/2008GL034563.

Najmi A., Milikh G., Secan J., Chiang K., Psiaki M., Bernhardt P., Briczinski S., Siefring C., Chang C.L., Papadopoulos K. (2014), Generation and detection of super small striations by F region HF heating, $J$. Geophys. Res. Space Phys., 119(7), doi:10.1002/2014JA020038.

Najmi, A., Eliasson, B., Shao, X., Milikh, G., and Papadopoulos, K. (2016), Simulations of ionospheric turbulence produced by HF heating near the upper hybrid layer, Radio Sci. 51, 704-717, doi:10.1002/2015 RS005866.

Oyama, S., B. Watkins, F. Djuth, M. Kosch, P. Bernhardt, and C. Heinselman (2006), Persistent enhancement of the HF pump-induced plasma line measured with a UHF diagnostic radar at HAARP, J. Geophys. Res., 111, A06309, doi:10.1029/2005JA011363.

Papadopoulos, K., R.R. Sharma, and V.K. Tripathi (1982), Parametric excitation of Alfvén waves in the ionosphere, J. Geoph. Res., 87, 1491.

Papadopoulos, K., and C. L. Chang (1985), Generation of ELF/ULF waves in the ionosphere by dynamo processes, Geophys. Res. Lett., 12, 279-281.

Papadopoulos. K., H.-B. Zhou and C.-L. Chang (1994), Cerenkov excitation of whistler/helicon waves by ionospheric HF heating, Geophys. Res. Lett., 21(17), 1767.

Papadopoulos, K., T. Wallace, M. McCarrick, G. M. Milikh, and X. Yang (2003), On the efficiency of ELF/VLF generation using HF heating of the auroral electrojet, Plasma Phys. Rep., 29, 561.

Papadopoulos, K., N. Gumerov, X. Shao, C.-L. Cheng, I. Doxas (2011a), HF driven current in the polar 
ionosphere, Geophys. Res. Lett., 38, L12103, doi:10.1029/2011GL047368.

Papadopoulos, K., C.-L. Chang, J. Labenski, and T. Wallace (2011b), First demonstration of HF driven ionospheric currents, Geophys. Res. Lett., 38, L20107, doi:10.1029/2011GL049263.

Pedersen, T. R., and H. C. Carlson (2001), First observations of HF heater produced airglow at the High Frequency Active Auroral Research Program facility: Thermal excitation and spatial structuring, Radio Sci., 36(5), 1013-1026, doi:10.1029/2000RS002399.

Pedersen, T., M. McCarrick, E. Gerken, C. Selcher, D. Sentman, H. Carlson, and A. Gurevich (2003), Magnetic zenith enhancement of HF radio-induced airglow production at HAARP, Geophys. Res. Lett., 30, 1169-1172, doi:10.1029/2002GL016096.

Pedersen, T.R., and E. Gerken (2005), Creation of visible artificial optical emissions in the aurora by highpower radio waves, Nature, 433, 498-500, doi:10.1038/nature03243

Pedersen, T., R. Esposito, E. Kendall, D. Sentman, M. Kosch, E. Mishin, and R. Marshall (2008), Observations of artificial and natural optical emissions at the HAARP facility, Ann. Geophys., 26, 10891099.

Pedersen, T., B. Gustavsson, E. Mishin, E. MacKenzie, H. C. Carlson, M. Starks, and T. Mills (2009), Optical ring formation and ionization production in high-power HF heating experiments at HAARP, Geophys. Res. Lett., 36, L18107, doi:10.1029/2009GL040047.

Pedersen, T., B. Gustavsson, E. Mishin, E. Kendall, T. Mills, H. C. Carlson, and A. L. Snyder (2010), Creation of artificial ionospheric layers using high-power HF waves, Geophys. Res. Lett., 37, L02106, doi:10.1029/ 2009GL041895. doi:10.1016/S0273-1177(02)00186-2.

Pedersen, T., M. McCarrick, B. Reinisch, B.Watkins, R. Hamel, and V. Paznukhov (2011), Production of artificial ionospheric layers by frequency sweeping near the 2nd gyroharmonic, Ann. Geophys., 29, 47-51, doi:10.5194/angeo-29-47-2011.

Perkins F.W., Oberman C., Valeo E.J. (1974), Parametric instabilities and ionospheric modification, $J$. Geophys. Res., 79(A10), 1478-1496.

Perkins F.W. (1974), A theoretical model for short-scale field-aligned plasma density striations, Radio Sci., 9(11), 1065-1070.

Piddyachiy, D., U. S. Inan, T. F. Bell, N. G. Lehtinen, and M. Parrot (2008), DEMETER observations of an intense upgoing column of ELF/VLF radiation excited by the HAARP HF heater, J. Geophys. Res., 113, A10308, doi:10.1029/2008JA013208.

Platino, M., U. S. Inan, T. F. Bell, M. Parrot, and E. J. Kennedy (2006), DEMETER observations of ELF waves injected with the HAARP HF transmitter, Geophys. Res. Lett., 33, L16101, doi:10.1029/2006GL026462.

Polyakov, S., and V. Rapoport (1981), The ionospheric Alfvén resonator, Geomagn. Aeron., 21, 816.

Ponomarenko P.V., Leyser T.B., Thide B. (1999), New electron gyroharmonic effects in HF scatter from pump-excited magnetic field-aligned ionospheric irregularities, J. Geophys. Res., 104(A5), 10,08110,087 .

Ponomarenko P. V., Yu. M. Yampolski, A. V. Zalizovsky, D. Hysell, and O. Tyrnov (2000), Interaction between artificial ionospheric irregularities and natural MHD waves, J. Geophys. Res., 105 (A1), 171181. 
Pradipta R., Lee M.C., Cohen J.F., Watkins D.J. (2015), Generation of artificial acoustic-gravity waves and traveling ionospheric disturbances in $\mathrm{HF}$ heating experiments, Earth, Moon, Planets,. doi: 10.1007/s11038-015-9461-2.

Rapoport V.O., Frolov V.L., Polyakov S.V., Komrakov G.P., Ryzhov N.A., Markov. G.A., Belov A.S., Parrot M., Rauch J.-L. (2010), VLF electromagnetic field structures in the ionosphere disturbed by SURA RF heating facility, J. Geophys. Res., 115, A10322, doi:10.1029/2010JA015484.

Reinisch, B., et al. (2001), First results from the Radio Plasma Imager on IMAGE, Geophys. Res. Lett. 28, 1167-1170, doi: 10.1029/2000GL012398.

Rich, F. J., and M. Hairston (1994), Large-scale convection patterns observed by DMSP, J. Geophys. Res., $79,3827$.

Rietveld, M., H.-P. Mauelshagen, P. Stubbe, H. Kopka, and E. Nielsen (1987), The characteristics of ionospheric heating-produced ELF/VLF waves over 32 hours, J. Geophys. Res., 92, 8707, doi:10.1029/JA092iA08p08707.

Rietveld, M. T., P. N. Collis and J.-P. St. Maurice (1991), Naturally enhanced ion-acoustic waves in the auroral ionosphere observed by the EISCAT 933 MHz radar, J. Geophys. Res., 96, 19291-19305.

Rietveld, M. T., H. Kohl, H. Kopka and P. Stubbe (1993), Introduction to ionospheric heating at Troms $\varnothing$. Experimental overview, J. Atmos. Terr. Phys., 55, 577-599.

Rietveld, M. T., B. Isham, H. Kohl, C. La Hoz, and T. Hagfors (2000), Measurements of HF-enhanced plasma and ion lines at EISCAT with high altitude resolution, J. Geophys. Res., 105 (A4), 7429-7439.

Rietveld, M. T., B. Isham, T. Grydeland, C. La Hoz, T. B. Leyser, F. Honary, H. Ueda, M. Kosch, T. Hagfors (2002), HF-pump-induced parametric instabilities in the auroral E-region, Adv. Space Res., 29, 9, 1363-1368.

Rietveld, M. T., M. J. Kosch, N. F. Blagoveshchenskaya, V. A. Kornienko, T. B. Leyser, T. K. Yeoman (2003), Ionospheric electron heating, optical emissions and striations induced by powerful HF radio waves at high latitudes: aspect angle dependence, J. Geophys. Res., 108 (A4), 1141, doi:10.1029/2002JA009543.

Rietveld, M. T., A. Senior, J. Markkanen, and A. Westman (2016), New capabilities of the upgraded EISCAT high-power HF facility, Radio Sci., 51, doi:10.1002/2016RS006093.

Robinson, T. (1989), The heating of the high latitude ionosphere by high-power radio waves, Phys. Rep., 179, 79-209.

Robinson, T. R. (1994), The Role of natural E-region plasma turbulence in the enhanced absorption of HF radio waves in the auroral ionosphere: Implications for RF heating of the auroral electrojet, Ann. Geophys., 12 (4), 316-332.

Robinson, T. R., F. Honary, A. Stocker, and T. B. Jones (1995), Factors influencing the heating of the auroral electrojet by high power radio waves, Adv. Space Res, 15 (12), 41-44.

Robinson, P. (1997), Nonlinear wave collapse and strong turbulence, Rev. Mod. Phys., 69, 507-573.

Robinson, T. R., G. Bond, P. Eglitis, F. Honary and M. T. Rietveld (1998), RF heating of a strong auroral electrojet, Adv. Space Res., 21 (5), 689-692.

Robinson, T. R., R. Strangeway, D. M. Wright, J. A. Davies, R. B. Horne, T. K. Yeoman, A. J. Stocker, M. Lester, M. T. Rietveld, I. R. Mann, C. W. Carlson, and J. P. McFadden (2000), FAST observations of ULF waves injected into the magnetosphere by means of modulated RF heating of the auroral electrojet, Geophys. Res. Lett., 27, 19, 3165-3168. 
Robinson, T. R., T. K. Yeoman, R. S. Dhillon, M. Lester, E. C. Thomas, J. D. Thornhill, D. M. Wright, A. P. van Eyken, and I. McCrea (2006), First observations of SPEAR induced artificial backscatter from CUTLASS and the EISCAT Svalbard radar, Ann. Geophys., 24, 291-309.

Rose, G., B. Grandal, E. Neske, W. Ott, K. Spenner, J. Holtet, K. Måseide, and J. Trøim (1985), Experimental results from the HERO Project: In situ measurements of ionospheric modifications using sounding rockets, J. Geophys. Res., 90, 2851-2860.

Ruzhin, Yu., et al. (2012), On the possibility of localization of a substorm by using the SURA heating facility, Radiophys. Quantum Elect, 55(1-2), 85-94.

Sagdeev, R. (1979), The 1976 Oppenheimer Lectures: Critical problems in plasma astrophysics, Rev. Mod. Phys., 51, 1-20.

Samimi, A., W. A. Scales, P. A. Bernhardt, S. J. Briczinski, and M. J. McCarrick (2013), Ion gyroharmonic structures in stimulated radiation during second electron gyroharmonic heating: 1. Theory, J. Geophys. Res. Space Phys., 118, 502-514, doi:10.1029/2012JA018146.

Samimi, A., W. A. Scales, P. A. Bernhardt, S. J. Briczinski, and M. J. McCarrick (2014), Ion gyroharmonic structures in stimulated radiation during second electron gyroharmonic heating: 2 . Simulations, $J$. Geophys. Res. Space Phys., 119, 462-478, doi:10.1002/2013JA019341.

Samson, J. C., D. D. Wallis, T. J. Hughes, F. Greutzberg, J. M. Ruohoniemi, and R. A. Greenwald (1992), Substorm intensifications and field line resonaces in the nightside magnetosphere, J. Geophys. Res., 97, 8495.

Sauvaud, J.-A., T. Moreau, R. Maggiolo, J.-P. Treilhou, C. Jacquey, A. Cros, J. Coutelier, J. Rouzaud, E. Penou and M. Gangloff (2006), High-energy electron detection onboard DEMETER: The IDP spectrometer, description and first results on the inner belt, Planet. Space Sci., 54, 502-511.

Scherbarth, M., A. Adler, and G. Ginet (2009), Demonstration and Science Experiment (DSX) Mission, Solar Physics and Space Weather Instrumentation III, eds. S. Fineschi, J. Fennelly, Proc. of SPIE v. 7438, 74380B-1-10, doi: 10.1117/12.824898.

Schlatter, N. M., N. Ivchenko, B. Gustavsson, T. Leyser, and M. Rietveld (2013), Observations of HF induced instability in the auroral E region, Ann. Geophys., 31, 1103-1108, doi:10.5194/angeo-31-11032013.

Schlatter, N. M., V. Belyey, B. Gustavsson, N. Ivchenko, D. Whiter, H. Dahlgren, S. Tuttle, and T. Grydeland (2015), Auroral ion acoustic wave enhancement observed with a radar interferometer system, Ann. Geophys., 33, 837-844, doi:10.5194/angeo-33-837-2015.

Schumann, W. (1952), On the damping of electromagnetic self-oscillations in the system earth-airionosphere, (in German), Zeitschrift für Naturforschung Z. Naturforschaftung 7,a 2, 250149-154, doi:10.1515/zna-1952-0202.

Scoffield, H., T. Yeoman, T. Robinson, L. Baddeley, R. Dhillon, D. Wright, T. Raita, and T. Turunen (2006), First results of artificial stimulation of the ionospheric Alfvén resonator at $78^{\circ} \mathrm{N}$, Geophys. Res. Lett., 33, L19, 103, doi:10.1029/2006GL027.

Sedgemore-Schulthess, K. J. F., and J.-P. St. Maurice (2001), Naturally enhanced ion-acoustic spectra and their interpretation, Surveys Geophys., 22, 55-92.

Senior, A., N. D. Borisov, M. J. Kosch, T. K. Yeoman, F. Honary, and M. T. Rietveld (2004), Multifrequency HF radar measurements of artificial F-region field-aligned irregularities, Ann. Geophys., 22, 3503-3511. 
Senior, A., F. Honary, P. J. Chapman, M. T. Rietveld, T. S. Kelso, and M. J. Kosch (2008), High-frequency magnetospheric sounding at EISCAT: some trials and their implications, Radio Sci., 43, RS4009, doi:10.1029/2007RS003779.

Senior, A., M.T. Rietveld, M.J. Kosch and W. Singer (2010), Diagnosing radio plasma heating in the polar summer mesosphere using cross-modulation: Theory and observations, J. Geophys. Res., 115, A09318, doi:10.1029/2010JA015379.

Senior, A., M.T. Rietveld, F. Honary, W. Singer, and M. J. Kosch (2011), Measurements and modelling of cosmic noise absorption changes due to radio heating of the D-region ionosphere, J. Geophys. Res., 116, A04310, doi:10.1029/2010JA016189.

Senior, A., M. T. Rietveld, I. Haggstrom, and M. J. Kosch (2013), Radio-induced incoherent scatter ion line enhancements with wide altitude extents in the high-latitude ionosphere, Geophys. Res. Lett., 40, 9, 1669-1674, doi:10.1002/grl.50272.

Senior, A., A. Mahmoudian, H. Pinedo, C. La Hoz, M. T. Rietveld, W. A. Scales, and M. J. Kosch (2014), First modulation of high-frequency polar mesospheric summer echoes by radio heating of the ionosphere, Geophys. Res. Lett., 41, 15, 5347-5353, doi: 10.1002/2014GL060703.

Sergeev, E.N., Frolov V.L., Komrakov G.P., Thide B. (1997), Temporal evolution of HF-excited plasma waves, measured at different pump frequencies by stimulated electromagnetic emission, J. Atmos. Solar-Terr. Phys., 59 (18), pp. 2383-2400.

Sergeev, E.N., Frolov V.L., Boiko G.N., Komrakov G.P. (1998), Results of investigation of the Langmuir and upper hybrid plasma turbulence evolution by means of the stimulated ionospheric emission, Radiophys. Quant. Electron., 41(3), 206-228.

Sergeev, E.N., Grach S.M., E.N., Komrakov G.P., Frolov V.L., Stubbe P., Thide B., Leyser T.B., Carozzi T.D. (1999), Influence of small-scale irregularities on characteristics of the overshoot effect in the temporal evolution of stimulated electromagnetic emission. Part I. Development stage, Radiophys. Quant. Electron., 42 (7), pp.544-556.

Sergeev, E.N. Grach S.M., E.N., Kotov P.V. (2004), Study of the excitation conditions and characteristics of ionospheric plasma turbulence at the development stage of the pondermotive parametric instability, Radiophys. Quant. Electron., 47(3), 185-204.

Sergeev, E.N., Frolov V.L., Grach S.M., Kotov P.V. (2006), On the morphology of SEE spectral features in a wide pump wave frequency range, Adv. Space Res., 38 (11), 2518-2526, doi: 10.1016/j.asr.2005.02.046.

Sergeev, E., S. Grach, A. Shindin, E. Mishin, P. Bernhardt, S. Briczinski, B. Isham, M. Broughton, J. LaBelle, and B. Watkins (2013), Artificial ionospheric layers during pump frequency stepping near the 4th gyroharmonic at HAARP, Phys. Rev. Lett., 110, doi:065002-1-5 0031-9007/13/110(6)/065002(5).

Sergeev, E.N., et al. (2016), Exploring HF-induced ionospheric turbulence by Doppler sounding and stimulated electromagnetic emissions at HAARP heating facility, Radio Sci., 51, doi:10.1002/2015RS005936.

Sergeev, E.N., Grach S.M., Frolov V.L., Shinding A.V. (2017), Diagnostics of the generation, decay, and transfer of artificial plasma perturbations by means of short pulses of a high-power radio emission, Radiophys. Quant. Electron., 59 (11), 876-887, doi:10.1007/s11141-017-9758-2.

Sergienko, T., I. Kornilov, E. Belova et al. (1997), Optical effects in the aurora caused by ionospheric HF heating, J. Atmos Sol.-Terr. Phys., 99(18), 2401-2407. 
Sergienko, T., B. Gustavsson, U. Brandstrom, and K. Axelsson (2010), Modelling of optical emissions enhanced by the HF pumping of the ionospheric F-region, Ann. Geophys., 30, 885-895, doi:10.5194/angeo-30-885-2012.

Shapiro, V., and V. Shevchenko (1984), Strong turbulence of plasma oscillations, in Basic Plasma Physics, A. Galeev and R. Sudan (eds), v. 2, 123-182, North-Holland, NY.

Shvarts M.M., Grach S.M., Sergeev E.N., Frolov V.L. (1995), On the generation of the stimulated electromagnetic emission. The computer simulation results, Adv. Space Res., 15(12), 59-62.

Siefring, C. L., P. A. Bernhardt, H. G. James, and R. T. Parris (2014), The CERTO beacon on CASSIOPE/ePOP and experiments using high-power HF ionospheric heaters, Space Sci. Rev., 189, 107-122, doi:10.1007/s11214-014-0110-2.

Simoes, F., R. Pfaff, H. Freudenreich (2011), Satellite observations of Schumann resonances in the Earth's ionosphere, Geophys. Res. Lett., 38(L22), 101, doi:10.1029/2011GL049668.

Starodubtsev, M., V. Nazarov, and A. Kostrov (2007), Laboratory study of nonlinear trapping of magnetized Langmuir waves inside a density depletion, Phys. Rev. Lett., 98, 195001-1-4, doi: 10.1103/PhysRevLett.98.195001.

Streltsov, A., W. Lotko, and G. Milikh (2005), Simulations of ULF field-aligned currents generated by HF heating of the ionosphere, J. Geophys. Res., 110, 629, doi:10.1029/2004JA010.

Streltsov, A.V., T.R. Pedersen, E.V. Mishin, and A.L. Snyder (2010a), Ionospheric feedback instability and substorm development, J. Geophys. Res., 115, A07205, doi:10.1029/2009JA014961.

Streltsov, A.V., M. Gołkowski, U.S. Inan, and K.D. Papadopoulos (2010b), Propagation of whistler-mode waves with a modulated frequency in the magnetosphere, J. Geophys. Res., 115, A09209, doi:10.1029/2009JA015155.

Streltsov, A.V. and T.R. Pedersen (2010), An alternative method for generation of ULF waves by ionospheric heating, Geophys. Res. Lett., 37, L14101, doi:10.1029/2010GL043543.

Streltsov, A.V. and T.R. Pedersen (2011), Excitation of zero-frequency magnetic field-aligned currents by ionospheric heating, Ann. Geophys., 29, 1147, doi:10.5194/angeo-29-1147-2011.

Streltsov, A.V., C.-L. Chang, J. Labenski, G. M. Milikh, A. Vartanyan, and A. L. Snyder (2011), Excitation of the ionospheric Alfvén resonator from the ground: Theory and experiments, J. Geophys. Res., 116, A10221, doi:10.1029/2011JA016680.

Streltsov, A.V., T. Guido, B. Tulegenov, J. Labenski, C.-L. Chang (2014), Artificial excitation of ELF waves with frequency of Schumann resonance, J. Atmos. Solar-Terr. Phys., 119, 110, doi:10.1016/j.jastp.2014.07.004.

Stubbe, P., and H. Kopka (1977), Modulation of the polar electrojet by powerful HF waves, J. Geophys Res., 82, 16, 2319-2325.

Stubbe, P., H. Kopka and R.L. Dowden (1981), Generation of ELF and VLF waves by polar electrojet modulation: Experimental results, J. Geophys. Res., 86 (A11), 9073.

Stubbe, P., H. Kopka, H. Lauche, M. T. Rietveld, A. Brekke, O. Holt T. B. Jones T. Robinson A. Hedberg B. Thidé B. Crochet and H.-J. Lotz (1982), Ionospheric modification experiments in northern Scandinavia, J. Atmos. Terr. Phys., 44 (12), 1025-1041.

Stubbe, P., H. Kopka, B. Thidé, and H. Derblom (1984), Stimulated electromagnetic emission: A new technique to study the parametric decay instability in the ionosphere, J. Geophys. Res., 89 (A9), 75237536. 
Stubbe, P., H. Kopka, M. T. Rietveld, A. Frey, and P. Hoeg, H. Kohl, E. Nielsen, G. Rose, C. LaHoz, R. Barr, H. Derblom, A. Hedberg, B. Thide, T. B. Jones, T. Robinson, A. Brekke, T. Hansen and O. Holt (1985), Ionospheric modification experiments with the Troms $\emptyset$ heating facility, J. Atmos. Terr. Phys., 47 (12), 1151-1163.

Stubbe, P., H. Kopka (1990), Stimulated electromagnetic emission in a magnetized plasma: A new symmetrical spectral feature, Phys. Rev. Lett., 65 (2), 183-186.

Stubbe, P., H. Kohl, and M. T. Rietveld (1992), Langmuir turbulence and ionospheric modification, $J$. Geophys. Res., 97, 6285--6297.

Stubbe, P., A. J. Stocker, F. Honary, T. R. Robinson, and T. B. Jones (1994), Stimulated electromagnetic emissions (SEE) and anomalous HF wave absorption near electron gyroharmonics, J. Geophys. Res., 99, 6233-6246.

Stubbe, P. (1996), Review of ionospheric modification experiments at Troms $\varnothing$, J. Atmos. Terr. Phys., 58, 1-4, 349-368.

Stubbe P., Hagfors T. (1997), The Earth's ionosphere: A wall-less plasma laboratory, Surveys in Geophysics, 18, (1), 57-127.

Sturman, B. (1974), Interaction of potential oscillations in a magnetoactive plasma, Radiophys. Quantum Electron., 17, 1349-1355.

Sulzer, M.P., J. D. Mathews, A. A. Tomko (1982), A UHF cross-modulation D region heating experiment with aeronomic implications, Radio Sci., 17 (2), 435-443.

Surkov,V., N. Nosikova, A. Plyasov, V. Pilipenko,V. Ignatov (2013), Penetration of Schumann resonances into the upper ionosphere., J. Atmos. Sol. Terr. Phys., 97, 65.

Tellegen, B. D. H. (1933), Interaction between radio waves, Nature, 131, 840, doi:10.1038/131840a0.

Tereshchenko, E.D., Khudukon B.Z., Gurevich A.V., Zybin K.P., Frolov V.L., Myasnikov E.N., Muravieva N.V., Carlson H.C. (2004), Radio tomography and scintillation studies of ionospheric electron density modification caused by a powerful HF-wave and magnetic zenith effect at mid-latitudes, Physics Lett., A, 325, 381-388.

Thidé, B., H. Kopka, and P. Stubbe (1982), Observations of stimulated scattering of a strong high frequency radio wave in the ionosphere, Phys. Rev. Lett. 49, 1561-1564, doi:10.1103/PhysRevLett.49.1561.

Thome G.D., Blood D.W. (1974), First observations of RF backscatter from field-aligned irregularities produced by ionospheric heating, Radio Sci., 9(11), 917-921.

Trakhtengertz, V., and A.Y. Feldstein (1984), Quiet auroral arcs: Ionospheric e®ect of magnetospheric convection stratification, Planet. Space Sci., 32, 127.

Trakhtengertz, V. Y., P. P. Belyaev, S.V. Polyakov, A. G. Demekhov, and T. Bosinger (2000), Excitation of Alfvén waves and vortices in the ionospheric Alfvén resonator by modulated powerful radio waves, J. Atmos. Terr. Phys., 62, 267.

Tripathi, V., C.L. Chang, and K. Papadopoulos (1982), Excitation of the earth-ionosphere waveguide by an ELF source in ionosphere, Radio Sci., 17(5), 1321.

Tsuda, T. T., M. T. Rietveld, M. J. Kosch, S. Oyama, K. Hosokawa, S. Nozawa, T. Kawabata, A. Mizuno, and Y. Ogawa, (2018), A survey of conditions for artificial aurora experiments at EISCAT Troms $\emptyset$ site using dynasonde data, Earth, Planets and Space, 70, 40, doi.org/10.5194/angeo-36-243-2018.

Uryadov, V.P., Vertogradov G.G., Vertogradov V.G., Komrakov G.P., Cherkashin Yu.N., Vas'kov V.V. (2007), Field-aligned scattering of HF radio waves under modification of the ionosphere by high-power 
oblique radio waves., Radiophys. Quant. Electr., 50(8), 611-618.

Vartanyan, A., G. M. Milikh, E. Mishin, M. Parrot, I. Galkin, B. Reinisch, J. Huba, G. Joyce, and K. Papadopoulos (2012), Artificial ducts caused by HF heating of the ionosphere by HAARP, J. Geophys. Res., 117, A10307, doi:10.1029/2012JA017563.

Vartanyan, A., G. M. Milikh, B. Eliasson, A. C. Najmi, M. Parrot, and K. Papadopoulos (2016), Generation of whistler waves by continuous HF heating of the upper ionosphere, Radio Sci., 51, 1188, doi:10.1002/2015RS005892.

Vas'kov, V. V., Ya. S. Dimant, and N. A. Ryabova (1993), Magnetospheric plasma thermal perturbations induced by resonant heating of the ionospheric F-region by high-power radio wave, Adv. Space. Res., 13(10), 25-33, doi:10.1016/0273-1177(93)90047-F.

Vas'kov, V. V., N. I. Bud'ko, O. V. Kapustina, Y. M. Mikhailov, N. A. Ryabova, G. L. Gdalevich, G. P. Komrakov, and A. N. Maresov (1998), Detection on the INTERCOSMOS-24 satellite of VLF and ELF waves stimulated in the topside ionosphere by the heating facility SURA, J. Atmos. Sol. Terr. Phys., 60(12), 1261-1274, doi:10.1016/S1364-6826(98)00054-6.

Vierinen, J., A. Kero, M. T. Rietveld (2013), High latitude artificial periodic irregularity observations with the upgraded EISCAT heating facility, J. Atmos. Sol.-Terr. Phys., 105-106, 253-261.

Vlasov, M.N., M. C. Kelley, and D. L. Hysell (2013), The main types of electron energy distribution determined by model fitting to optical emissions during HF wave ionospheric modification experiments, J. Geophys. Res., 118, 3877-3890, doi:10.1002/jgra.50364.

Volokitin, A., and E. Mishin (1979), Relaxation of an electron beam in a plasma with infrequent collisions, Sov. J. Plasma Phys., 5, 654.

Volokitin, A., and C. Krafft (2005), Wave decay processes in a weakly magnetized plasma, Physics Letters A, 336, 193-198.

Wang, J., D. Newman, and M. Goldman (1997), Vlasov simulations of electron heating by Langmuir turbulence near the critical altitude in the radiation-modified ionosphere, J. Atmos. Sol. Terr. Phys., 59, 2461, doi:10.1016/S1364-6826(96)00140-X.

Wong, A. Y., J. Carroll, R. Dickman, W. Harrison, W. Huhn, B. Lure, M. McCarrick, J. Santoru, C. Schock, G. Wong, and R. F. Wuerker (1990), High-power radiating facility at the HIPAS Observatory, Radio Sci., 25 (6), 1269-1282.

Wright, D. M., J. A. Davies, T. K. Yeoman, T. Robinson, S. R. Cash, E. Kolesnikova, M. Lester, P. J. Chapman, R. J. Strangeway, R. B. Horne, M. T. Rietveld, C. W. Carlson (2003), Detection of artificially generated ULF waves by the FAST spacecraft and its application to the "tagging" of narrow flux tubes, J. Geophys. Res., 108 (A2), 1090, doi:10.1029/2002JA009483.

$\mathrm{Xi}, \mathrm{H}$., and W. Scales (2001), Numerical simulation studies on the broad upshifted maximum of ionospheric stimulated electromagnetic emission, J. Geophys. Res., 106, 12,787, doi:10.1029/2000JA000322.

Zabotin, N.A, and Kovalenko, E.S. (1999), Simple numerical model of radio wave multiple scattering effects in the ionospheric plasma layer, Waves in Random Media, 9(3), 393-399, doi: 10.1088/09597174/9/3/307.

Zakharov, V. (1984), Collapse and self-focusing of Langmuir waves, in Basic Plasma Physics, vol. 2, A. Galeev and R. Sudan (Eds.), pp. 81-121, North-Holland, New York.

Zhou, H. L., Huang, J., and Kuo, S. P. (1994), Cascading of the upper hybrid/electron Bernstein wave in ionospheric heating experiments, Phys. Plasmas 1, 3044-3052. 